Michael Fink

\title{
Soziale Sicherung im Spannungsfeld gesellschaftlicher Transformation
}

Eine partizipative Studie in Küstendörfern der Fidschi-Inseln

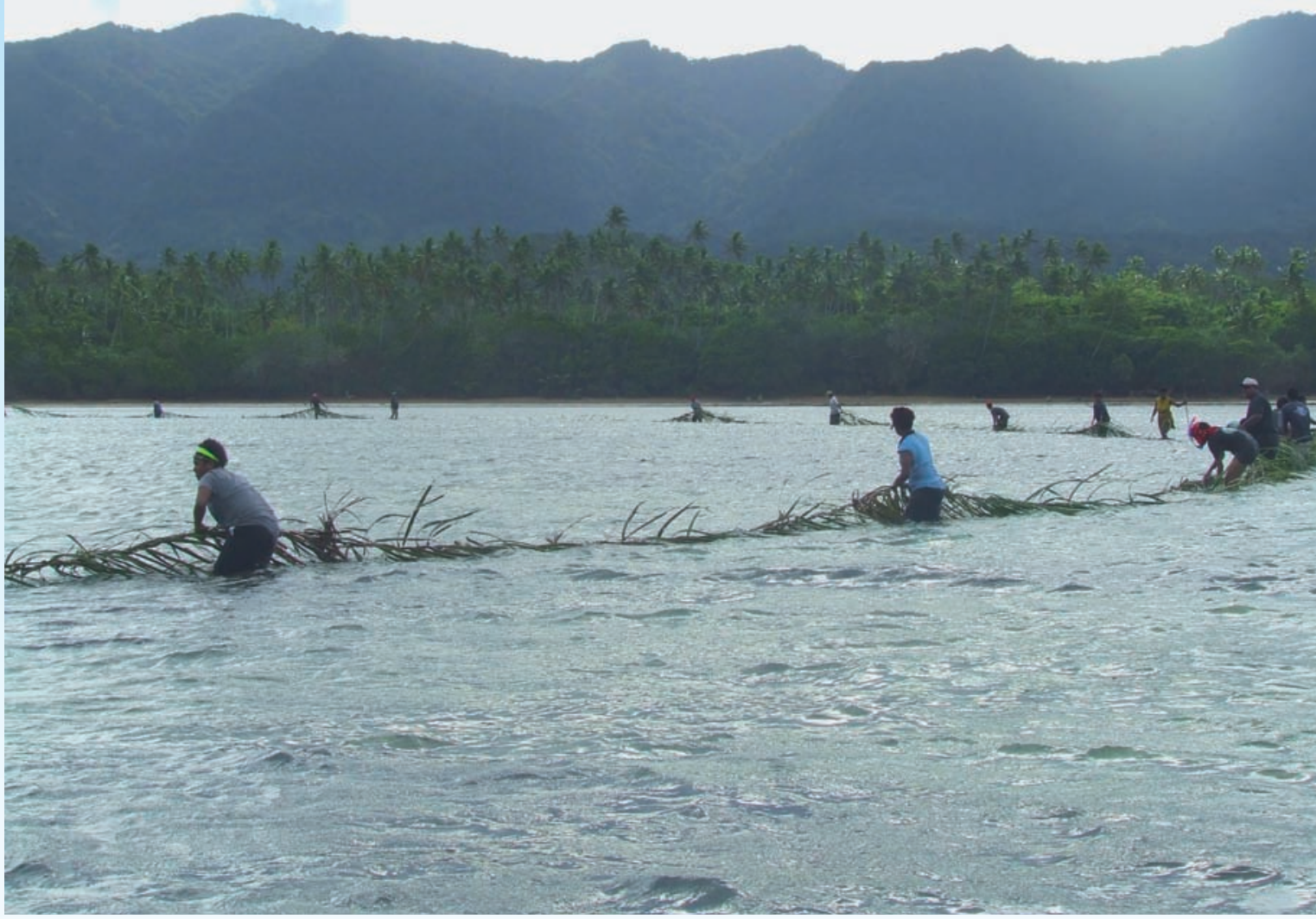

\section{Pazifik Forum}

\section{Band 17}



Michael Fink

Soziale Sicherung im Spannungsfeld gesellschaftlicher Transformation

Dieses Werk ist lizenziert unter einer

Creative Commons

Namensnennung - Weitergabe unter gleichen Bedingungen

4.0 International Lizenz.

(c) (†) (?) 
erschienen im Universitätsverlag Göttingen 2016 
Michael Fink

Soziale Sicherung im Spannungsfeld gesellschaftlicher Transformation

Eine partizipative Studie in Küstendörfern der Fidschi-Inseln

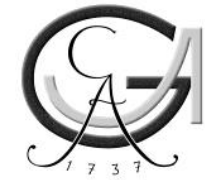

Universitätsverlag Göttingen 2016 


\section{Bibliographische Information der Deutschen Nationalbibliothek}

Die Deutsche Nationalbibliothek verzeichnet diese Publikation in der Deutschen Nationalbibliographie; detaillierte bibliographische Daten sind im Internet über $<$ http://dnb.dnb.de $>$ abrufbar.

Anschrift des Autors

Michael Fink

E-Mail: mifink85@googlemail.com

Dieses Buch ist auch als freie Onlineversion über die Homepage des Verlags sowie über den Göttinger Universitätskatalog (GUK) bei der Niedersächsischen Staats- und Universitätsbibliothek Göttingen (http://www.sub.uni-goettingen.de) erreichbar. Es gelten die Lizenzbestimmungen der Onlineversion.

Titelabbildung:

Traditionelle Fischtreibjagd auf Gau Island, Fidschi. (C) Eberhard Weber 2009

Satz und Layout: Michael Fink

(C) 2016 Universitätsverlag Göttingen http:/ / univerlag.uni-goettingen.de

ISBN: 978-3-86395-261-7 


\section{Arbeitsgemeinschaft für $\mathbf{P a z i f i s c h e ~ S t u d i e n ~}$}

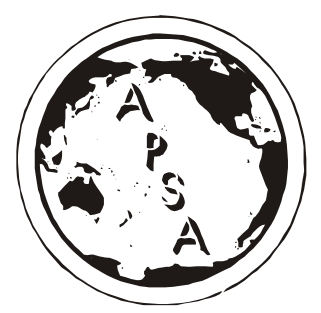

Michael Fink

\section{Soziale Sicherung im Spannungsfeld gesellschaftlicher Transformation.}

Eine partizipative Studie in Küstendörfern der Fidschi-Inseln

Hrsg.: Christoph Dittrich

Heiko Faust

Michael Waibel 



\section{Vorwort}

Die vorliegende Arbeit befasst sich mit dem Leben fidschianischer Küstendorfbewohner ${ }^{1}$ und deren Wünschen, Hoffnungen, Ängsten und Sorgen. Da ich mich dafür in eine mir zuvor kulturell und naturräumlich fremde Umgebung begeben habe, bis dahin in Städten und deren Vororten in Deutschland lebte, wollte ich mir nicht anmaßen, diese im Voraus zu kennen. In ihrer Diversität bleibt sie mir sicherlich bis heute verborgen. Für die vorliegende Untersuchung ging ich daher explorativ und induktiv vor, versuchte einen möglichst offenen Forschungsprozess zu gestalten und keine vorschnellen Schlüsse zu ziehen, um die Lebensrealität der Menschen bestmöglich zu fassen. Oberste Prämisse dieser Arbeit ist es, über ein Thema von hoher Relevanz für die Menschen vor Ort zu schreiben, um etwas Sinnvolles für die dortigen Menschen zu erbringen. Über ein Studium mit der Schwerpunktsetzung Geographischer Entwicklungsforschung und einer Ausbildung in partizipativen Methoden wurde einerseits eine Basis geschaffen, dieses Ziel erreichen zu können, andererseits wird diese Rahmung meinen Blick auf das Wesentliche auch eingegrenzt haben.

Meine zentralen Anfangsfragen waren zunächst, was die Menschen bewegt und warum es sie bewegt. Um dies nachvollziehen zu können, wollte ich insbesondere den kulturellen Wertehorizont verstehen. Daher zielte ein Großteil der Forschung darauf ab, mich in den Dorfkontext einzuleben. Von rund 14 Monaten auf den Fidschi-Inseln verbrachte ich über die Hälfte der Zeit in vorwiegend drei Dorfgemeinschaften. Über eine Kurzdarstellung meines Zugangs zu den Dorfgemeinschaften möchte ich erste Einblicke in die örtliche Lebenswelt und erfahrene Werte geben und die Grundmotivation hinter dieser Arbeit verdeutlichen. Aus dieser wird auch ersichtlich, warum anschließend eine Danksagung folgt.

\section{Exkurs 1: Zugang zu fidschianischen Dorfgemeinschaften}

Fidschianische Dörfer sollte man nach Möglichkeit nicht ohne Einladung betreten, da dies einen kulturellen Affront darstellt. In einem nicht näher bestimmbaren „Früher“ wurden Ungebetene häufig verjagt, ggfs. sogar getötet. Dörfer betritt man - dies ist bewusst idealisierend formuliert - mit einem Gastgeschenk und einem Fürsprecher. Daraufhin wird eine Willkommenszeremonie (sevusevu) veranstaltet, bei der man vom Fürsprecher dem Häuptling als pars pro toto der Gemeinschaft vorgestellt wird. Zudem wird das Gastgeschenk vom Fürsprecher überreicht. Der Sprecher des Häuptlings wiederum nimmt dieses im Namen des Häuptlings und damit stellvertretend für die Dorfgemeinschaft an und heißt einen in deren Namen willkommen. Gefestigt wird die Praxis durch das gemeinsame Trinken von

\footnotetext{
${ }^{1}$ Sofern Informationen über das Geschlecht einer Person keinen Mehrwehrt für die Forschungsergebnisse zu beinhalten scheinen, wird in dieser Arbeit auf die Kenntlichmachung verzichtet und das generische Maskulinum verwendet.
} 
yaqona, einem Getränk mit spirituellem Beiklang, damit auch die Ahnen dem Aufenthalt ihren Segen geben. Anschließend informiert der Sprecher die nicht-anwesenden Haushalte über den Gast im Dorf, dessen Hintergrund und Anliegen.

Die Wahl meiner Untersuchungsdörfer hing nicht nur an erkenntnistheoretischen Erwartungen und monetären wie zeitlichen Machbarkeiten, sondern wurde auch von der Auffindbarkeit geeigneter Fürsprecher in der Hauptstadt Suva beeinflusst. In allen Fällen fand ich hervorragende Fürsprecher, was ich über die Ankunft in mein erstes Untersuchungsdorf, Malawai auf Gau Island, verdeutlichen will. Dies ist das Heimatdorf von Joeli Veitayaki, Professor für Marine Studies an der University of the South Pacific (USP) und ich bin mit ihm als Fürsprecher gemeinsam angereist. Im Rahmen eines sevusevu in der Gemeindehalle stellte er mich, meine Herkunft, mein familiäres Umfeld und mein Forschungsanliegen dem Häuptling, seinem Sprecher und weiteren Anwesenden vor und überreichte ein Bündel yaqona-Wurzeln als Gastgeschenk. Der Sprecher des Häuptlings nahm das Geschenk an und hieß mich in der Gemeinde willkommen. Er erklärte mir, dass der Häuptling sich geehrt fühle und mir große Freiheiten im Dorf gewähre, dass ich beispielsweise wahllos von jedem Baum die Früchte pflücken dürfe, mich an den Feldern bedienen dürfe, mich frei auf dem des Dorfes zugehörigen Land und Wasser bewegen dürfe und mir jedes Haus zu jeder Tages- und Nachtzeit offen stünde. Nach der Zeremonie verkündete er meine Anwesenheit den anderen Dorfbewohnern. Da nun jeder von mir wusste und allem Anschein nach ein sehr positives Bild von mir hatte, war eine ideale Basis für meine partizipative Forschung geschaffen, ohne dass ich bisher ein einziges Wort hätte sagen müssen.

Gespräche mit meinen Forschungsassistenten ergaben, dass man mir den gleichen Respekt und das gleiche Vertrauen entgegenbringt wie meinem Fürsprecher. Man vertraut darauf, dass ich meine Freiheiten nicht überstrapaziere und auf Kosten anderer ausnutze. Sollte ich Schaden anrichten, so würde dieser in erster Linie nicht mir angelastet, sondern meinem Fürsprecher, weil er mich ins Dorf geholt hat, und dem Häuptling, der mich mit den Freiheiten und Möglichkeiten ausgestattet hat, die Dorfgemeinschaft zu belasten. Emotional fühle ich mich seitdem beiden Menschen sehr verbunden, bin sehr dankbar für den Vertrauensvorschuss, der mir gegeben wurde und möchte dieses Vertrauen nicht enttäuschen.

Eine Grundmotivation dieser Niederschrift ist es, etwas zurückzugeben, nachdem inzwischen so viele Menschen - die mir über die Zeit sehr ans Herz gewachsen sind - so viel für mich getan haben, Fleiß, Geduld und Rücksichtnahme in mich investiert haben und Hoffnung in mich stecken. Das zentrale Anliegen der vorliegenden Studie ist es, Erkenntnisse zu erzielen, die die Lebensqualität dieser Menschen bewahren und möglicherweise sogar steigern.

\section{Danksagung}

$\mathrm{Zu}$ allererst möchte ich den Menschen und Gemeinschaften danken, die mich so offen und nachsichtig, warmherzig und liebevoll aufgenommen haben. Stellvertretend für die Dörfer möchte ich den Häuptlingen danken, sowie den Familien, unter deren Dächern ich lebte. Vielen Dank an Tui Malawai, Tui Nabuna, Tui 
Lamiti und Tui Bucabuca; sowie Tevita, Pita, Liti, Epeli, Alumita, Ringo, Vilikesa, Sera und Sosi. Eure Freundschaften haben mein Leben ungemein bereichert.

Eberhard Weber und Joeli Veitayaki von der USP, euch beiden gilt ein ganz besonderer Dank. Eure Kompetenzen und eure selbstlose Hilfsbereitschaft haben mir nicht nur den Einstieg in diese wundervolle neue Welt eröffnet, sondern ihr seid stetige, vertrauensvolle Ansprechpartner. Ohne die vielen klärenden Gespräche mit meinen Forschungsassistenten wären meine Einblicke in die örtliche Lebenswelt partikularer geblieben. Ich danke Oni, Vilisi, Maleli, Eri, Loraini, Livai und Milika.

Ebenso gilt mein Dank auch meiner Familie und meinen Freunden in Deutschland, ohne deren vielseitige Unterstützung das Projekt weder gestartet noch beendet worden wäre. Dabei danke für die kritische Durchsicht dieser Schrift vor allem Vincent Brenner, Alexander Opitz und Thomas Wieland.

Wenn ich von dieser Dissertationsschrift als Arbeit spreche, so steht der Begriff Arbeit nicht für Qual und Mühe. Ich empfinde es als eine Ehre, über dieses Medium Erkenntnisse, Erfahrungen und Ansichten einer interessierten Leserschaft anvertrauen zu dürfen. ${ }^{2}$ Für die Schaffung dieser Möglichkeit und Unterstützung bedanke ich mich bei meinen Betreuern Christoph Dittrich und Heiko Faust, sowie bei der gesamten Abteilung Humangeographie der Universität Göttingen für das angenehme wie inspirierende Arbeitsumfeld. Ich danke Elfriede Hermann und Markus Keck für die sorgfältige Durchsicht und konstruktive Kritik. Für die Hilfe zur Erstellung der Karten bedanke ich mich bei Andreas Flemnitz. Zuletzt möchte ich noch Herrn Hans-Georg Bohle gedenken, über dessen Förderung und Wertschätzung ich sehr dankbar bin.

\footnotetext{
${ }^{2}$ Das Verfassen einer Dissertation stellt jedoch natürlich eine Herausforderung dar. Ich habe sie daher in meiner Muttersprache verfasst, um mich sprachlich möglichst sicher und exakt ausdrücken zu können. In Form von wissenschaftlichen Artikeln und Präsentationen in englischer Sprache möchte ich wesentliche Erkenntnisse dieses Buches auch der örtlichen Bevölkerung zugänglich machen und habe dies bereits in Ansätzen getan (Fink 2010a; Fink 2012; Veitayaki \& Fink 2012).
} 



\section{Inhaltsverzeichnis}

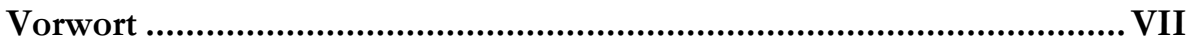

Inhaltsverzeichnis ..................................................................................

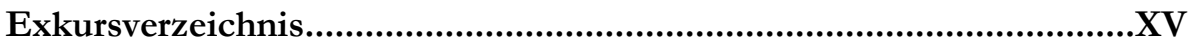



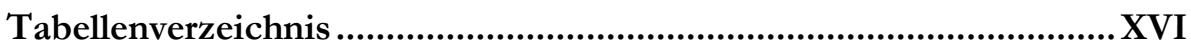

Kartenverzeichnis ........................................................................... XVI

Glossar und Abkürzungsverzeichnis .....................................................XVI

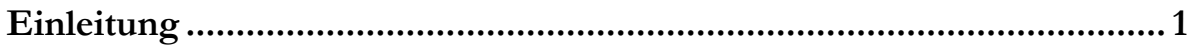

A Methodologie ........................................................................ 9

1. Partizipation als Forschungsprinzip in interkulturellen Kontexten .11

1.1 Relevanz des Forschungsthemas, Auswahl der Methodik und des

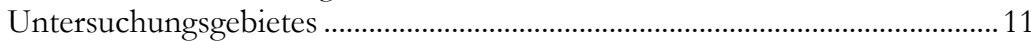

1.2 Ethik in der interkulturellen Forschung ....................................................... 12

1.2.1 Vereinbarkeit von Ethik und Wissenschaft bei partizipativer Forschung...

12.

1.2.2 Forschung als persönlicher Prozess mit kritischer Selbstreflektion .......... 14

1.3 Partizipation in der Forschung und Entwicklungspraxis.................................. 15

1.3.1 Intersubjektivität im interkulturellen Kontext............................................... 17

1.3.2 Verzerrungen entwicklungspolitischer Forschung .................................... 17

1.3.3 Minimierung von Verzerrungen mittels Partizipation ................................19

1.3.4 Umgang mit Verzerrungen aufgrund der eigenen Persönlichkeit............. 20

1.4 Macht und Vertrauen in partizipativer Forschung ............................................2 21

1.4.1 Machtpolitische Dimensionen von Partizipation......................................... 22

1.4.2 Qualität von Gruppenarbeiten und Visualisierungen...................................22

1.5 Aufbau der Forschungsmethoden .................................................................... 25

1.6 Angewandte partizipative (PRA-) Methoden.......................................................22



1.7.1 Limitationen der Güte der Forschung ....................................................... 32

1.7.2 Machtungleichheiten und mangelnder Partizipationsgrad bei Gruppendiskussionen und Visualisierungen................................................... 33

1.7.3 Zur Rechtfertigung von Machtungleichgewichten in der Forschung ....... 34

1.7.4 Überwindungsversuche des mangelnden Grades an Partizipation............. 36

B Theoretisch-konzeptioneller Rahmen .................................... 38

2. Die Gabe - Einführung in kulturtheoretische Grundbegriffe ......... 40

2.1 Relationales Raumverständnis ....................................................................... 41 


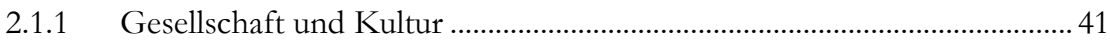

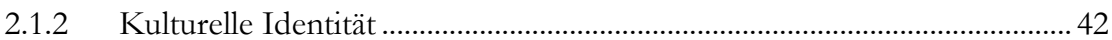

2.1.3 Transkulturalität statt Multikulturalität ....................................................... 44

2.1.4 Transdisziplinäre Erfassung des Wertefundaments kollektiver Identitäten

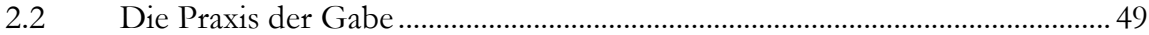

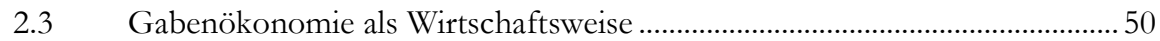

$2.4 \quad$ Soziale Beziehungen und Sozialkapital .......................................................... 53

3. Globalisierung und gesellschaftliche Transformation......................58

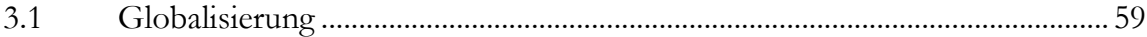



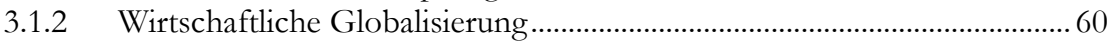

3.1.3 Sozio-kulturelle Globalisierung und neo-traditionelle Gegenbewegungen

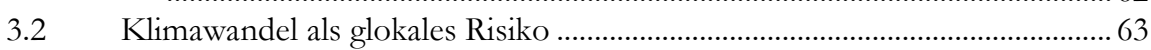

3.2.1 Wissenschaftliche Grundlagen des Klimawandels........................................64 64

3.2.2 Auswirkungen des Klimawandels auf menschliche Gesellschaften ......... 66

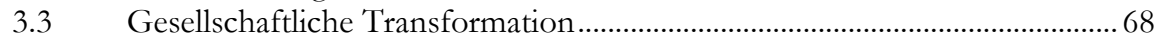

3.3.1 Globalisierung als Motor heutiger Transformationsprozesse .................... 70

3.3.2 Transformation als Unsicherheit ................................................................. 71

4. Lebensqualität - Soziale Sicherung durch Verwirklichungschancen..



4.1 Eurozentristische Vorstellungen von Armut und Entwicklung....................... 73

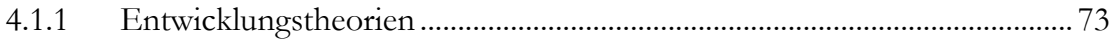

4.1.2 Nachhaltige Entwicklung ............................................................................. 75

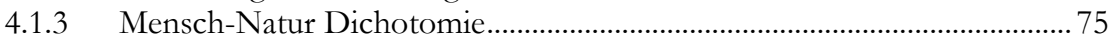

4.1.4 Kultur, Entwicklung und die Entzauberung der Welt................................ 77





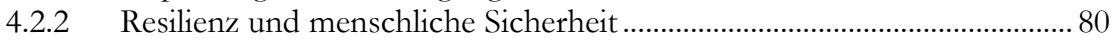

4.3 Menschliche Sicherung durch Verwirklichungschancen .................................. 81

4.4 Ein integratives Konzept zu sozialer Sicherung............................................. 84

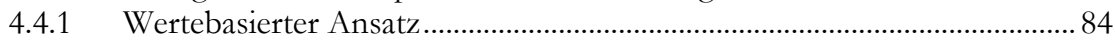

4.4.2 Demokratie als öffentlicher Vernunftgebrauch ......................................... 85

C Kulturräumlicher Überblick ...................................................89

5. Fidschi im Zeichen gesellschaftlicher Transformation .................... 90

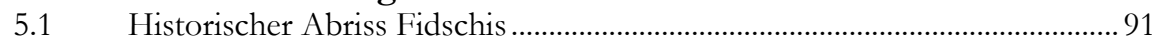

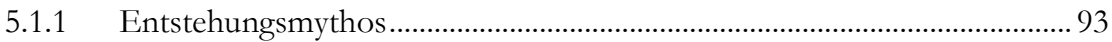

5.1.2 Präkoloniale Ära ........................................................................................ 95

5.1.3 Anfänge der kolonialen Ära - Landrecht und indische Kontraktarbeiter....

5.1.4 Ethnische Spaltungen im Zuge der Kolonialpolitik .................................. 99 
5.2 Gesellschaftliche Spannungsfelder und politische Konfliktlinien seit der



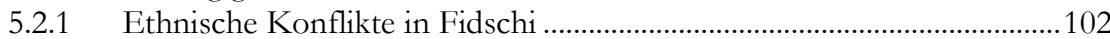

5.2.2 Konflikte entlang der Häuptlingskonföderationen....................................105

5.2.3 Konfliktfelder und Lösungsansätze in der Militärdiktatur .......................107

5.3 Exposition gegenüber Klimawandel und Naturgefahren ..............................110

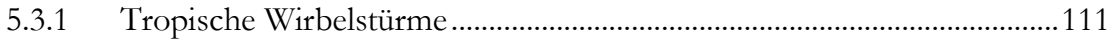

5.3.2 Physische Auswirkungen des Klimawandels ..............................................112

5.4 Gesellschaftliche Trends und Dynamiken ....................................................114

DEmpirie - Darstellung und Analyse ...................................... 118

6. Einführung in die untersuchte Lebenswelt - kulturelle Identität ..120

6.1 Politisch-administrative Ordnungseinheiten und soziale Identitäten...........121



6.1.2 Die Großfamilie ........................................................................................ 122

6.1.3 Dörfliches Zusammenleben ...................................................................125

6.1.4 Konfliktpotentiale aufgrund von institutionellem Pluralismus ...............126

6.2 Malawai: Sozial-ökonomische Einblicke......................................................127

6.2.1 Marktwirtschaftliche Beziehungen ............................................................ 130

6.2.2 Häuptlingswahl und Hexerei ...................................................................132

6.2.3 Migration als Chance und Risiko der Transformation...............................136

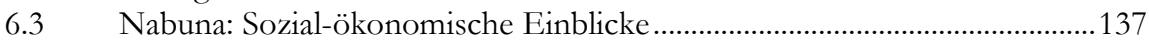

6.3.1 Marktwirtschaftliche Beziehungen und Reziprozität ................................141

6.3.2 Soziale Gliederung und Häuptlingswesen ..................................................143

6.3.3 Einbindung in traditionelle regionale Systeme .........................................145

6.3.4 Risiken und Chancen der Transformation.................................................146

6.4 Dravuni: Sozial-ökonomische Einblicke ...........................................................147

6.4.1 Einbindung in politische, traditionelle und kirchliche Gliederungssysteme

6.4.2 Marktwirtschaftliche Beziehungen und lokale Entwicklungsprojekte 152

6.4.3 Gruppenbildung aus politisch-administrativen Zwängen .........................154

6.5 Sozialräumliche Dimensionen von Familiennetzwerken..................................155

7. Vanua - Die räumliche Manifestation der kulturellen Identität.....160

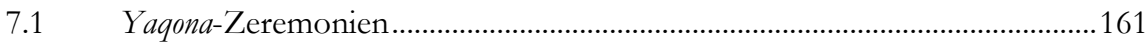



7.1.2 Respekt gegenüber den Ahnen...................................................................... 164

7.2 Vanua im Zeichen der Transformation - Neo-traditionelle Bewegungen ........

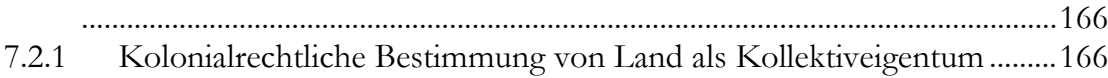

7.2.2 Yaqona-Zeremonien zwecks Fundraising ..................................................167

7.2.3 Vereinbarkeit des christlichen Glaubens mit vanua ...................................168

7.2.4 Wirtschaftliche Inwertsetzung des Landes - Zugang zu Land als



7.2.5 Touristische Niederlassung auf Koro Island ...............................................172

7.2.6 Generationenkonflikt beim Reinigen der Friedhöfe .................................173

7.2.7 Traditionelle Fischtreibjagd als Gegenbewegung......................................174 
7.2.8 Monasavu: Besetzung eines Staudammprojektes......................................177

7.3 Vanua als Essenz des Kulturellen (Zwischenfazit) .......................................... 178

7.4 Tabu als lokale Antwort auf den globalen Nachhaltigkeitsdiskurs ................ 179

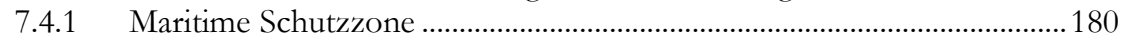



8. Lebensqualität in fidschianischen Küstendörfern ........................183

8.1 Einklang im Dreiklang - mentale, physische und spirituelle Fitness als



8.1.1 Projektion des Dreiklangs guten Lebens in gesellschaftlichem Oberbau 184

8.1.2 Intergenerationelle Dimensionen eines guten Lebens ...............................185

8.1.3 Störungen eines guten Lebens aufgrund gesellschaftlicher Transformation

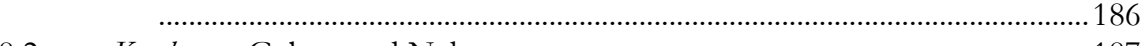

8.2 Kerekere - Geben und Nehmen ................................................................... 187

8.2.1 Mythos einer einseitigen Ausnutzung aufgrund gesellschaftlicher

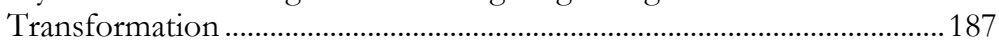

8.2.2 Anpassungsfähigkeit Dank öffentlicher Diskussion ..................................188

8.3 Klimawandelanpassung und Naturgefahrenvorsorge .................................... 191

8.3.1 Herausforderungen bei der Andockung neuer Wissenssysteme an

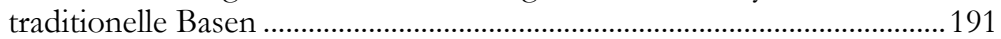

8.3.2 Erfahrungen mit Zyklonen in Malawai und physisch-materielle

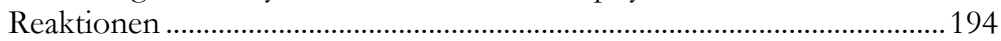

8.3.3 Soziale Sicherung als Anpassung an Naturgefahren...................................197



Literaturverzeichnis ............................................................... 207






\section{Exkursverzeichnis}

Exkurs 1: Zugang zu fidschianischen Dorfgemeinschafte .................................................... VII

Exkurs 2: Weihnachten 2009 - Malawai, Gau Island ...........................................................129

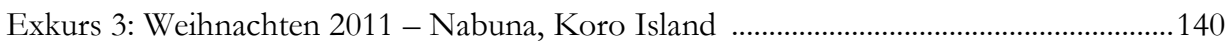

Exkurs 4: Weihnachten 2012 - Dravuni, Tailevu, Viti Levu................................................... 148

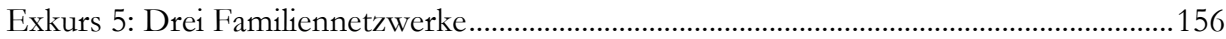

\section{Abbildungsverzeichnis}

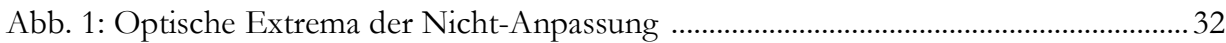

Abb. 2: Bevölkerungszusammensetzung hinsichtlich Religionen - Stand 2007 .................. 106

Abb. 3: Vergleich ruraler und urbaner Bevölkerungspyramiden ...........................................115

Abb. 4: Zusammensetzung eines Drei-Generationen-Haushalts ........................................122

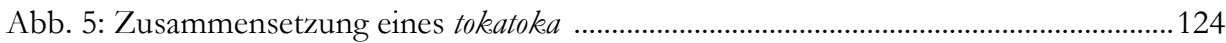

Abb. 6: Venn-Diagramm sozialer Institutionen der männlichen Jugend Malawais ...........131

Abb. 7: Formaler Aufbau und Kommunikationsstruktur in Malawai ...................................132

Abb. 8: Zeitstrahl der Dorfgeschichte Malawais .......................................................................133

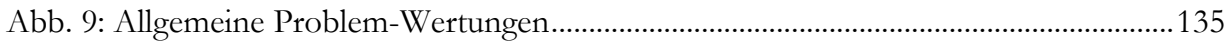

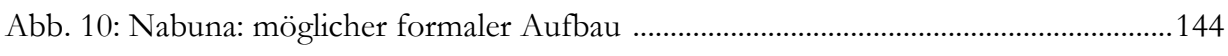

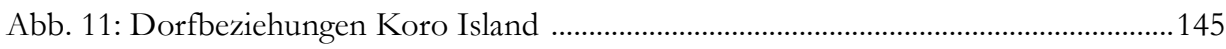

Abb. 12: Schematische Sitzrunde in Yaqona-Zeremonie .....................................................162



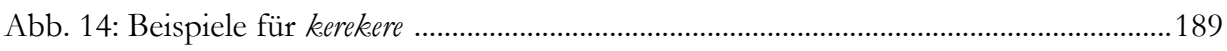

Abb. 15: Vor- und Nachteile von kerekere.........................................................................189

Abb. 16: Auswirkungsdiagramme tropischer Zyklone ............................................................194

Abb. 17: Bleibende Verwüstungen durch Zyklon Tomas (Vorher - Nachher) .................196 


\section{Tabellenverzeichnis}

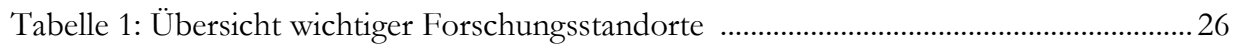

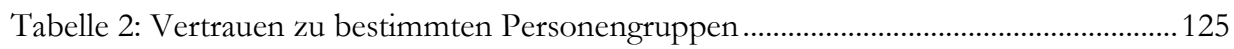

Tabelle 3: Soziale Beeinflussung individueller Handlungsspielräume ..................................159

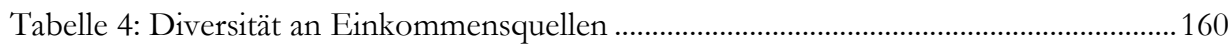

\section{Kartenverzeichnis}

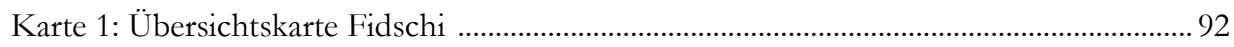

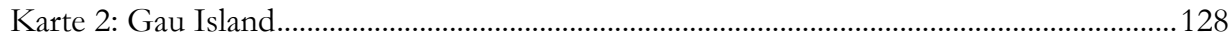

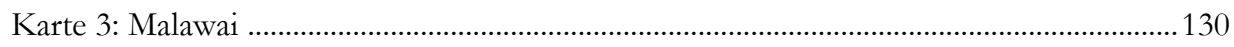



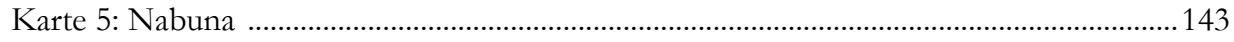

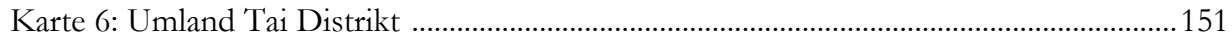

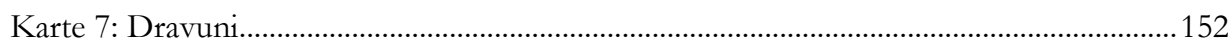

Karte 8: Drei Familiennetzwerk-Kartierungen ....................................................................... 157

\section{Glossar und Abkürzungsverzeichnis}

$\begin{array}{ll}\text { bati } & \text { Krieger-Funktion im Häuptlingswesen } \\ \text { bete } & \text { Priester- bzw. Diener-Funktion im Häuptlingswesen } \\ \text { BMZ } & \text { Bundesministerium für wirtschaftliche Zusammenarbeit und } \\ & \text { Entwicklung } \\ \text { bilo } & \text { Kokosnussschale, aus der yaqona getrunken wird } \\ \text { boca ni vanua } & \text { traditionelle Germeindeversammlung } \\ \text { bure } & \text { traditionelles Haus aus Holz und Blättern } \\ \text { Burebasaga } & \text { südliche Konföderation } \\ \text { CAR } & \text { Forschungsmethodenschule zu gemeindebasiertem Forschen und } \\ & \text { Handeln (community action research) } \\ \text { cumu } & \text { Drückerfisch, traditionell den bati vorbehalten } \\ \text { dau } & \text { Begabten-Funktion im Häuptlingswesen (z.B. als gonedau, Fischer } \\ & \text { oder dau ni vucu, Poet) }\end{array}$


EZ Entwicklungszusammenarbeit

FLMMA lokal betreute maritime Schutzzone (Fijian locally managed marine area)

GCC Großer Häuptlingsrat (Great Council of Chiefs)

grog

gunu

kava

siehe yaqona

trinken, Getränk

kerekere

siehe yaqona

Kubuna

eine Bitte, die man nicht abschlagen darf

lotu

mana

zentrale Konföderation

mata ni vanua

Religion, Spiritualität, oftmals synonym für die christliche Kirche göttliche, alles Leben durchdringende Kraft

matai

Herald-Funktion im Häuptlingswesen, Sprecher des Häuptlings (wortwörtlich etwa: Auge des Landes)

matanitu Gesellschaftsordnung, kann b

Spezialisten-Funktion im Häuptlingswesen (z.B. als mataisau, Zimmermann)

mata ni tikina Distrikt-Verwalter

mataqali Subklan

MPA

PLA

maritime Schutzzone (marine protected area)

Forschungsmethodenschule zu partizipativem Lernen und Handeln (participatory learning and action)

PRA Forschungsmethodenschule zu partizipativer, ländlicher Projektplanung (participatory rural appraisal)

qoliqoli exklusiver Fischgrund des zugehörigen Dorfes

Ratu hoher Adelstitel, notwendige Bedingung zum Beitritt in den GroBen Häuptlingsrat

sau ni vanua hoher Titel, der übernatürliche Fähigkeiten nahelegt (wörtlich: der Segen des Landes)

sauturaga Häuptlingsmacher-Funktion im Häuptlingswesen

sevusevu traditionelle Willkommenszeremonie

SIDS kleine, wirtschaftsschwache Inselstaaten (Small Island Developing States)

sinu kulturell bedeutende Baumart mit giftigen Blättern

USP Universität Südpazifik (The University of the South-Pacific)

UN Vereinte Nationen (United Nations)

tabu heilig, geweiht

talatala Pfarrer, Vorsitzender einer Kirchengemeinde

tanoa große Holzschale zum Ausschenken von yaqona

iTaukei

Kurzform von Taukei ni qele, Besitzer des Bodens bzw. Landbesitzer; Selbstbezeichnung der autochtonen Fidschianer

tawn

Menschen aus als miteinander befreundet geltenden Provinzen, weshalb sie besondere Gastrechte genießen 
TLTB

tokatoka

Tovata

tugadra

Tui

turaga

turaga ni koro

vanua

vaka i taukei

vakatana

vakaturaga

vakavanua

vasu

vulagi

WBGU

yaqona

yavirau

yavusa
Institution zur Regelung von Pachtverträgen (iTaukei Land Trust Board)

Großfamilie

nordöstliche Konföderation

Totem des Dorfes, Schwarmfisch (wahrscheinlich Makrelengattung Selar crumenophthalmus) der im Schnitt etwa alle 10 Jahre an Malawai vorbeizieht; durchschnittlicher Fang: 3000-6000, von denen traditionell drei Viertel an Nachbardörfer verschenkt werden

Adelstitel

Häuptlings-Funktion im Häuftlingswesen

gewählter Dorfvorsteher

Kernbegriff räumlicher und kultureller Identifikation (wörtlich u.a.: Land oder Stammeszusammenschluss)

neo-traditionelle Gegenbewegung zu westlichen Entwicklungsidealen, die politisch instrumentalisiert wird (in der Literatur wird meist nur die englische Bezeichnung "The Fijian W ay of Life" verwendet)

ausgebildeter Laiepriester innerhalb einer Dorfgemeinschaft edel, königlich

normativ hochwertig, kulturell wertvoll (wörtlich etwa: nach Art des Landes)

Neffe oder Nichte; Nachfahre dem im mütterlichen Heimatdorf besondere Ehren zuteil werden

Gast, Ausländer

Wissenschaftlicher Beirat der Bundesregierung Globaler Umweltwandel

Nationalgetränk Fidschis, Mixtur aus gleichnamiger Pfefferwurzel (piper methysticum) und Wasser mit leicht betäubender Wirkung, auch kava oder grog genannt; ritualisiert getrunken dient es als Medium zur Kommunikation mit den Ahnen

traditionelle Fischtreibjagd

Stamm 


\section{Einleitung}

Diese Dissertation ordnet sich in die gesellschaftswissenschaftliche und kulturtheoretische Humangeographie ein. Weder die behandelten sozialen Phänomene, noch deren Konzeptualisierung oder methodische Erfassung sind ausschließlich humangeographischer Natur, werden jedoch vorwiegend aus einer humangeographischen Betrachtung durchleuchtet und problematisiert. Die Arbeit versteht sich als Beitrag, welcher die Humangeographie mit inter- und transdisziplinären Perspektiven bereichern soll. Innerhalb der Humangeographie bedient sie sich insbesondere Perspektiven der Geographischen Verwundbarkeitsforschung und der Neuen Kulturgeographie und verortet sich regional im Südpazifik. Den methodischen Ansatz kennzeichnet eine aktive, weitreichende und verantwortungsvolle Beteiligung der Menschen vor Ort. Er ist als partizipativ und ethnographisch zu bezeichnen. Die geographische Betrachtungsweise ergänzt er dabei um soziologische, philosophische, politikwissenschaftliche und vor allem ethnologische Komponenten.

Forscht man als Europäer über gesellschaftswissenschaftliche Themen im interkulturellen Kontext, wird man unweigerlich mit Diskussionen um „Entwicklung“ bzw. „Entwicklungszusammenarbeit“ (EZ) konfrontiert. Kaum ein Begriff prägt so zentral unser Denken der letzten 70 Jahre über die unterschiedlichsten Regionen unserer Welt. Nach diesem Interpretationsraster befinden sich diese auf einem Kontinuum zwischen „entwickelt“ und „unterentwickelt“. Auch wenn dies zumeist in subtileren Kategorien wie „reich“ und ,arm“, „gebildet“ und ,ungebildet“, „Nord“ und „Süd“ oder „Westen“ und „Rest der Welt", „,frei“ und „unfrei“", ,umweltfreundlich“ und „umweltzerstörerisch“, sowie zunehmend in „sicher“ und ,unsicher" geschieht; ein Verständnis von Entwicklung ist stets konnotiert. Kaum ein Konzept ist in der Wissenschaft so vielseitig diskutiert und umstritten wie das Narrativ von Entwicklung (Eckert 2015: 3; Nuscheler 2005: 11-16). So stellt sich auch die Geographie der Frage, ob sie unter Labeln wie „Entwicklungsforschung“ oder „Entwicklungsländerforschung“ in diesen Kontexten agieren kann bzw. dürfe (Korf \& Rothfuss 2016: 164-166).

Diese Arbeit möchte die interkulturelle, sozialgeoraphische Forschung um kritische Perspektiven bereichern. Insbesondere der Vorwurf des Eurozentrismus im Kontext von Entwicklung wird kritisch reflektiert, um diesbezügliche Problematiken zu lösen oder zumindest zu reduzieren. Eurozentrismus ist eine spezifisch europäische oder nordamerikanisch-westeuropäische Form eines Ethnozentrismus. Dieser beschreibt eine Haltung gegenüber Menschen einer anderen Kultur, basierend auf der in der eigenen Kultur erlernten Wahrnehmungs-, Wertungs- und Verhaltensmuster. Zumeist werden die eigenen Normen dabei als universelles und überlegenes Bewertungsmuster verklärt. Zur Messung halten dann meist solche Indikatoren, Kategorien und Maßstäbe her, in denen westliche Staaten besser abschneiden als die übrige Welt. Im Sinne der Kritik aus Perspektive des Postkolonialismus und Postdevelopmentalismus sind westliche Normen im Zuge des 
Soziale Sicherung im Spannungsfeld gesellschaftlicher Transformation

Kolonialismus global wirkungsmächtig geworden und degradieren nicht-westliche Kulturen als rückständig. Sie untergraben das Selbstverständnis ihrer Angehörigen (Escobar 1995; Haller 2010: 17, 35; Honer 2007: 203; Melber 1992; Müller \& Ziai 2015: 8f.; Peet \& Hartwick 2011: 197-230).

Für diese Arbeit suchte ich daher einen Zugang zur Forschung, der so frei wie möglich von westlich geprägten Denkmustern und Wertvorstellungen gedacht sein soll. Dennoch kann ich meine eigene kulturelle und soziale Herkunft und persönliche Wissensbasis nicht ausschalten. In einem Miteinander mit den Dorfbewohnern wollte ich ein Thema von möglichst hoher Relevanz für die Menschen vor Ort erarbeiten. Auch wenn diese Forschung nicht mit einer ausformulierten Leitfrage startete, begann ich mit folgender Intention: Was bedeutet gutes Leben ${ }^{3}$ für fidschianische Küstendorfbewohner?

Methodisch wird ein offener und unvoreingenommener Ansatz gewählt, um die Anliegen fidschianischer Küstendorfbewohner, um deren Wünsche, Ängste und Hoffnungen möglichst unverfälscht aufnehmen und wiedergeben zu können. Partizipative, ethnographische Methoden bieten sich bei einer offenen Forschungsfrage und einem ganzheitlichen Interesse an (McQuiston et al. 2005: 210; Verne 2012: 186f.). Ich habe daher einen partizipativen Ansatz entwickelt, um den ethischen wie empirischen Dimensionen der Forschung möglichst gerecht zu werden. Den Startpunkt der Forschung bildeten lediglich die Region und eine Vorauswahl partizipativer Methoden zur anfänglichen Gewinnung von Zugang und Vertrauen zur Dorfgemeinschaft und zur Überblicksverschaffung der für sie relevanten Themen. Dabei wurde beständig kritisch reflektiert, ob ,gutes Leben“ kein relevantes Thema sei.

Einerseits sollen die Dimensionen guten Lebens möglichst ganzheitlich erfasst und wiedergegeben werden. Ansichten und Belange, die sich klassischen wissenschaftsdisziplinären Betrachtungsweisen entziehen oder der Lebensrealität des Autors oder der Leserschaft widersprechen könnten, - beispielsweise Aspekte wie Spiritualität oder Magie, - werden nicht ausgeblendet oder abqualifiziert. Andererseits muss diese offene, weitläufige Frage auf eine im Rahmen dieser Dissertationsschrift bearbeitbare Größe heruntergebrochen werden.

Schnell wurde deutlich, dass es in dieser Studie inhaltlich um gesellschaftliche Rahmenbedingungen, Normen und Werte geht, aus denen sich Leitbilder für gutes Leben ableiten lassen. Die Entstehung sowie vor allem der Wandel von Werturteilen und Gerechtigkeitsvorstellungen stehen im Fokus der Studie. Die Wirkmächtigkeit westlicher Entwicklungsnarrative im heutigen Fidschi wird dabei kritisch durchleuchtet. Im Verlauf des Forschungsprozesses konnten die benannten Wünsche und Ziele, Probleme und Herausforderungen miteinander verknüpft

\footnotetext{
${ }^{3}$ Der Ausdruck ,gutes Leben“ soll möglichst konzeptfrei verstanden sein. Er orientiert sich nicht am lateinameri-kanischen Gedanken von ,buen vivir“", welcher bestimmte Lebensstile und Entwicklungsideale preist (Acosta 2009: 219-22; Walsh 2010: 18-20).
} 
und im Themenkomplex der vorliegenden Studie, „Soziale Sicherung im Spannungsfeld gesellschaftlicher Transformation“ strukturiert gebündelt werden.

\section{Einführung ins Spannungsfeld gesellschaftlicher Transformation auf den Fidschi-Inseln}

Die Fidschi-Inseln gelten, wie andere pazifische Inselstaaten, als ein Entwicklungsland voller Gefahrenpotenziale und als Verwundbarkeits-Hot Spot. Von den Auswirkungen des globalen Umweltwandels seien sie in besonderem Maße negativ betroffen. Unter den Bewohnern des Pazifiks seien ländliche Küstenbewohner besonders verwundbar. In Semi-Subsistenzwirtschaft lebend und entsprechend von Fischfang und Feldarbeit geprägt, überbeanspruche die wachsende Bevölkerung die maritimen und ländlichen Ressourcen und setze sie Umweltbelastungen aus. Aufgrund des Klimawandels steige der Meeresspiegel an und Küstenerosion und Bodenversalzungen nähmen zu. Zusätzlich intensivierten sich bestehende Naturrisiken wie tropische Zyklone (Chand \& Walsh 2009: 3878; Connell 2013: vii, 17f.; Villagrán De León 2006: 32-36; Mataki et al. 2008: 264-265). So könnte Zyklon Pam, der im März 2015 Vanuatu traf und Auswirkungen bis Fidschi hatte, die größte humanitäre Naturkatastrophe in der Geschichte des Südpazifiks sein. Sollten die benannten Gefahren im befürchteten Maße zutreffen, würde es für die betroffenen Gesellschaften entsprechend hohe Anpassungs- und Bewältigungsvermögen erfordern, um diese Bedrohungen erfolgreich zu meistern.

Neben akuten Risiken seitens der nicht-menschlichen Umwelt kennzeichnet die Fidschi-Inseln ein gesellschaftlicher Transformationsprozess. Seit dem 19. Jahrhundert durchlief das Inselreich von einer vorkolonialen Zeit über eine knapp 100jährige koloniale Phase bis zur Unabhängigkeit 1970 massive gesellschaftliche Veränderungen. Seit ihrer Loslösung von der britischen Kolonialmacht existiert kein stabiles politisches System. Begleitet von bisher vier Putschversuchen schwankt das Land zwischen Diktaturen und demokratieähnlichen Regierungsgebilden. Aufgrund der geringen Gesamtbevölkerung und wirtschaftlichen Schwäche, eines attestierten Demokratiedefizits, sowie der nur mäßig interessanten geostrategischen Lage findet Fidschi kein Gehör in internationalen politischen Diskursen (Baldacchino 2009: 29-32; Briguglio 1995: 1615; Lewis 2009: ix-xiv).

In die heutige, postkoloniale Ära wirkt nicht nur die eigene Vergangenheit hinein, im Zuge der Globalisierung sind weite Bevölkerungsteile Fidschis in weltpolitische und weltwirtschaftliche Geschehnisse eingebunden, welche gesellschaftliche Veränderungen bewirken. Teile der Fidschi-Inseln sind beliebte Orte internationaler Touristen. Speziell über das Militär als UN-Blauhelme, im Auslandskontingent der britischen Armee oder in privaten Sicherheits- und Militärunternehmen sowie über Arbeitsmöglichkeiten auf Frachtschiffen erlangen viele Fidschianer Auslandserfahrungen. Konfrontiert mit westlichen, urbanen Lebensweisen und Wertesystemen durchläuft die ursprünglich dörfliche Kultur einen beschleunigten Wandel. 
Im Südpazifik finden sich überweltdurchschnittliche Verstädterungsraten ${ }^{4}$ mit entsprechenden gesellschaftlichen Veränderungen. Vor 15 Jahren zumeist noch nicht existent, prägen Kinos, Nachtlokale, Einkaufszentren und internationale Schnellrestaurant-Ketten inzwischen das Stadtbild. Die Kultur ist ein hybrides Gebilde, die ein Spannungsverhältnis zwischen traditionellen, inkludierten gegenüber westlichen, dualistischen Vorstellungen von Mensch, Geist und Natur kennzeichnet. Die Fidschianer befinden sich in einer Phase rasanter, unübersichtlich gewordener, gesellschaftlicher Veränderungen. Einschlägige Literatur zu gesellschaftlichen Transformationsprozessen legt nahe, dass derart komplexe Phänomene für die betroffene Bevölkerung kaum hinreichend zu verstehen seien. Deren Auswirkungen auf ihren Alltag seien ebenfalls unklar, sodass leicht Gefühle der Unsicherheit entstehen (Bohle 2001; Dittrich 2004; Polanyi 1944/2001; Tröger 2003).

Eine gesellschaftliche Transformationsphase gilt insbesondere für ohnehin besonders verwundbare Teile der Bevölkerung als von erhöhter Unsicherheit gekennzeichnet. Es kommt mit den Verschiebungen von Werten zu Gewinnern und Verlierern, zur Inklusion und Exklusion, und traditionelle Sicherungssysteme fallen schneller weg als sich neue etablieren können. Jedoch ist eine gesellschaftliche Transformation nicht einseitig negativ zu betrachten. Mit der Intensivierung der globalen Vernetzung wandeln sich kulturelle Praktiken. Dabei können sich individuelle wie gesellschaftliche Handlungsspielräume weiten, beispielsweise in ökonomischer Hinsicht, die für eine Verbesserung der gesamt-gesellschaftlichen Situation sprechen.

\section{Zielsetzung und Aufbau der Arbeit}

Das Forschungsinteresse dieser Arbeit gilt nicht nur einem rein wissenschaftlichtheoretischen Erkenntnisgewinn, sondern erweitert sich um ethische und entwicklungsfördernde Dimensionen. Der Aufbau folgt weitestgehend klassischen humangeographischen Darstellungsprinzipien: vom Globalen zum Lokalen, vom Strukturellen zum Konkreten, vom Allgemeinen zum Speziellen, von der Theorie zur Praxis - und zurück. Aufgrund der besonderen Herangehensweise werden jedoch Abweichungen zu einem typischen Aufbau in Kauf genommen. Im Gegensatz zu (sozial-)theoriegeleiteter humangeographischer Forschung wird nicht von einem theoretischen Überbau großer (europäischer, männlicher, weißer) Denker aus die Forschung konzipiert. Stattdessen verlangt der ethnographische Zugang, den diese Arbeit für die interkulturelle Humangeographie zugänglich machen will, zuerst eine Erklärung der Methodologie, bevor eine theoretischkonzeptionelle Rahmung vorgestellt wird. Zudem soll über den Einbau kleiner

\footnotetext{
${ }^{4}$ Der Verstädterungsgrad Fidschis stieg in den letzten 50 Jahren von $18 \%$ auf $53 \%$, weltweit von $34 \%$ auf 54\%. Fidschis urbane Bevölkerung versiebenfachte sich, weltweit vervierfachte sie sich im Vergleichszeitraum (eigene Berechnungen nach UN Department of Economic and Social Affairs 2014: 7, 11; Faust 1996: 24; UN Statistics Division 2015).
} 
Exkurse zum kulturellen Setting und kritischer Selbstreflektion in Ich-Perspektive den partizipativen, ethischen wie ethnographischen Ansprüchen (HauserSchäublin 2003: 50f.; Reithofer 2009: 358) auch im Schriftbild Rechnung getragen werden.

In methodischer Hinsicht stellt sich die Frage, wie die Forschung konzipiert sein kann, damit sie den wissenschaftlichen, ethischen und entwicklungspraktischen Ansprüchen dieser Studie gerecht werden kann. Dieser Einleitung folgt diesbezüglich im ersten Kapitel zuerst die Vorstellung und Rechtfertigung der weitestgehend partizipativen Methodenkombination. Die Vorzüge und Beeinträchtigungen partizipativer Forschung werden hier ausgeführt. Um der Leserschaft die Möglichkeit einer Überprüfung der Stimmigkeit des Forschungsdesigns zu schaffen, wird die Forschungsmethodik entsprechend detailliert dokumentiert und begründet.

In dieser normativen Betrachtungsweise sind Zahlen und Fakten zu Tatbeständen der Lebenssituation, beispielsweise hinsichtlich Einkommen, Armut und deren Verteilung, sowie Bildungs- und Aufstiegschancen oder der Verfügbarkeit und des Zugangs zur Nutzung natürlicher Ressourcen eine nützliche Basis. Darüber hinaus bedarf es jedoch insbesondere eines normativen Maßstabs, die Qualität der Lebenssituation beurteilen zu können. Diese Maßstäbe und Werturteile entspringen dabei den Vorstellungen der untersuchten küstendörflichen fidschianischen Gesellschaft bzw. haben sich im partizipativen Miteinander in den besuchten fidschianischen Dorfgemeinschaften entwickelt.

Das inhaltliche Ziel dieser Arbeit ist es herauszufinden, was gutes Leben für fidschianische Küstendorfbewohner ausmacht und wie dieses erhalten und gesteigert werden kann. Daher werden Auswirkungen einer Transformation explizit auf die Lebenssituation menschlicher Individuen - und somit lokal - dargestellt und erklärt, sowie Praxisbeispiele von Steuerungsversuchen anhand soziokultureller Wertmaßstäbe evaluiert. Erreicht wird dies, indem - vor einem theoretischen Hintergrund zu gesellschaftlichen Transformationsprozessen - Verwundbarkeiten sowie Anpassungsmaßnahmen sozialer Sicherung zur Überwindung jener Verwundbarkeiten partizipativ herausgefunden werden.

Im Anschluss an die Methodologie folgen dafür zunächst drei Kapitel zur theoretisch-konzeptionellen Einbettung der Arbeit. Die induktive, ethnographische Forschung versteht sich als ein Prozess des Verstehens. Hierfür muss auch der theoretische Zugang zunächst offen gestaltet sein. Theorien können Potentiale einer Erkenntnis sowohl ermöglichen, als auch verstellen. Entsprechend konnten die letztlich verwendeten Konzepte zur Unterlegung der empirischen Befunde erst im Nachhinein nach kritischer Reflektion final ausgewählt und sinnentsprechend zusammengestellt werden. Nach Julia Verne hinterfrage diese Umkehrung, nicht von einem theoretischen Überbau ausgehend die Forschung zu konzipieren, das derzeit vorherrschende Ideal nach einer vorgeschalteten sozialtheoretischen Fundierung in der Humangeographie (2012: 192f.). Die Studie ist damit jedoch keineswegs theoriefrei. Im Gegenteil werden theoretische Erkenntnisse so nuanciert 
zusammengestellt, dass sie dem Verständnis der Sache dienen. Die theoretischen Konzepte dienen dem Ziel, die örtlichen Lebensrealitäten entsprechend der soziokulturell bedingten Eigen- und Fremdwahrnehmung interpretieren zu können. Dieses ethnographische Theorieverständnis wird in der geographischen Entwicklungsforschung zunehmend angewendet und auch diese Arbeit erhofft sich, die interkulturell angelegte humangeographische Forschung über den gewählten theoretischen Zugang zu bereichern.

Chronologisch wurden die Erkenntnisse im Sinne induktiver, nichteurozentristischer Forschung möglichst vorbehaltsfrei bzw. aus sich selbst heraus erzielt und anschließend bzw. parallel mit der Literatur diskutiert. Zum besseren Verständnis dieser Dissertation sieht die Gliederung vor, sich zuerst konzeptionell anzunähern, bevor darauf aufbauend die kulturspezifischen empirischen Befunde diskutiert und interpretiert werden. Der eigene Erkenntnisgewinn wird demnach aufgrund der induktiven, partizipativen Herangehensweise achronologisch widergespiegelt.

Die verwendete Literatur schließt explizit Werke nicht nordamerikanischer oder westeuropäischer Autoren ein und hinterfragt punktuell den wissenschaftlichen Mainstream. Ich möchte bestehendes eurozentrisches Wissen dekonstruieren. Diese Diskussion findet sich bereits im theoretisch-konzeptionellen Rahmen und setzt sich über die gesamte Arbeit fort. Der Erkenntnisgewinn ist von prozessualem Charakter und methodologisch benötigte es einen fortwährenden, kritisch reflektierenden Literaturabgleich, um die Erlebnisse wissenschaftlich aufbereiten zu können. Für diese Studie wird daher die Literatur diskutiert, welche am hilfreichsten erscheint, die Erfahrungen darzustellen und zu verarbeiten, wissenschaftlich einzubetten und zu verwerten. Tautologien, erzielte Ergebnisse seien aufgrund des theoretischen Zugangs vorweggenommen, wird Dank des ethnographischen Zugangs entgegengewirkt.

Zur theoretischen Aufbereitung des Begriffs des „guten Lebens“ greift diese Arbeit zentral auf das Lebenswerk des indisch-stämmigen Wirtschaftshistorikers und Philosophen Amartya Sen zurück, der diesbezüglich von ,Quality of Life“ bzw. „Lebensqualität" spricht. ${ }^{5}$ Zur Annäherung an die Grundthematik, wie Lebensqualität im Zuge gesellschaftlicher Veränderungen bewahrt und gesteigert werden kann, werden zuerst gesellschaftswissenschaftliche Grundbegriffe erklärt. Begriffe wie „Gesellschaft“, „Kultur“ und „Identität“ werden vorgestellt und deren Raumwirksamkeit herausgestellt. Klarheit über die eigene, kulturelle Identität kann demnach als eine Quelle von Sicherheit und Wohlbefinden angesehen werden. Menschen werden als soziale Wesen konzipiert, deren Lebensqualität entscheidend von ihrem sozialen Umfeld abhängt. Die Praxis der Gabe, welche explizit in

\footnotetext{
${ }^{5}$ Ich hielt mich zum ersten Mal im Rahmen meiner Magisterarbeitsstudie zu „Verwundbarkeit gegenüber Naturgefahren“ (Fink 2010a; Fink 2010b) auf den Fidschi-Inseln auf. Gutes Leben bzw. Wohlbefinden, verstanden als Lebensqualität, kristallisierte sich hier bereits als zentrales Themenfeld heraus. Im hiesigen Forschungsverlauf wurde dies beständig kritisch hinterfragt, jedoch gab es zu keiner Zeit einen Anlass, deren Bedeutung anzuzweifeln.
} 
pazifischen Kulturen angewendet wird und dort erstmals aus anthropologischer Perspektive theoretisch aufbereitet wurde, wird als eine soziale Wirtschaftsweise vorgestellt, die das Gemeinwesen stärkt. Darauf folgend wird theoretisiert wie Globalisierungs- und Transformationsprozesse auf soziale Räume wirken. Das für diese Studie entscheidende Charakteristikum ist das Einhalten marktwirtschaftlicher Wirtschafts- und letztlich Denkweisen. Der Einzug kapitalistischer Ideale in nicht-westliche Kulturen vollzieht sich dabei nicht konfliktfrei und kann dazu führen, dass Gesellschaften sich entzweien und ein soziales Miteinander konterkariert wird. Zuletzt entwickle ich ausgehend von Überlegungen zu „Entwicklung“ und über die Konzepte „Soziale Verwundbarkeit“ und „Menschliche Sicherheit“ ein integratives Konzept, welches soziale Sicherung als Handlungsstrategie zur Gewinnung von Lebensqualität begreift. Die Möglichkeiten zu öffentlichem Vernunftgebrauch - also soziale Praktiken der Interaktion und Kommunikation um Werte und Ansichten zu schärfen und zu teilen - stellen das zentrale Mittel dar, menschliche Sicherheit und Lebensqualität zu erwirken.

Nach der Methodologie und der theoretisch-konzeptionellen Einbettung folgt im dritten Abschnitt ein Überblick über das Forschungsgebiet. Einerseits werden Prozesse gesellschaftlichen Wandels aus einer vorwiegend historischen Perspektive analysiert und als Transformation charakterisiert. Andererseits werden naturräumliche Herausforderungsdimensionen hinsichtlich eines Klimawandels und Naturgefahren vorgestellt. Dieser Abschnitt umreißt damit die strukturellen Rahmenbedingungen, wie auf Fidschi dem Ziel einer Lebensqualität nachgegangen werden kann.

Basierend auf den theoretisch-konzeptionellen Vorüberlegungen und auf dem sozio-kulturellen Vorverständnis der Ausgangslage besteht der letzte Abschnitt aus drei Kapiteln zu den empirischen Befunden vor Ort. Zuerst werden die drei Hauptuntersuchungsdörfer vorgestellt. Das soziale Zusammenleben und diesbezügliche Facetten des Wandels werden im Hinblick auf die Schaffung kultureller Identität zur Sicherung von Lebensqualität erörtert. Zur Vertiefung des kulturellen Verständnisses wird danach der Begriff vanua als Schlüsselbegriff für die Beziehung der Menschen zu ihrer Umgebung ergründet. Ein tiefgreifendes Verständnis über das kulturell geprägte Raumverständnis der Fidschianer befähigt dazu, die Dimensionen der Chancen und Herausforderungen der Transformation ganzheitlicher zu begreifen: Wo liegen Gefahren und wo sind Handlungsräume zur Überwindung von Verwundbarkeiten verortet? Zuletzt wird explizit der Frage nachgegangen, was Lebensqualität für die Küstendorfbewohner bedeutet. Möglichkeiten, Lebensqualität zu erzeugen, werden gesellschaftsinhärent ausschließlich im sozialen Miteinander gesehen. Sämtliche Phänomene, ob Naturereignisse oder gesellschaftliche Veränderungen werden demnach nur zur Gefahr, sofern sie die Gemeinschaft bedrohen. Die Studie kommt zu dem Ergebnis, dass, unter der Voraussetzung der Wahrung eines festen Wertefundaments, die gesellschaftliche Transformationen aufgrund der Erweiterung der Handlungspotentiale eine Bereicherung für fidschianische Küstendorfgemeinschaften darstellt. 


\section{Ausblick, gebende Vorbemerkungen}

In erster Linie betreibt diese Studie Grundlagenforschung - Fragen nach „was“ und „warum“ stehen im Vordergrund. Das Wissen, welches diese Arbeit zu produzieren und reproduzieren erhofft, soll dabei indirekt Möglichkeiten einer praktischen Anwendung schaffen. Bestehende Handlungsstrategien werden analysiert und ihr Erfolg auf Basis emischer Wertmaßstäbe begründet. Die Studie zeigt damit nicht nur Potentiale zur gerechten Gestaltung der Globalisierung auf, sondern analysiert bereits existierende Strategien, die vor Ort angewendet werden und Erfolg erzielen. Ich tätige jedoch bewusst keine politischen Handlungsempfehlungen; Fragen nach einem „wie“ zur Umsetzung künftiger EZ wird nicht gesondert nachgegangen. Gerade auch im Hinblick auf die Gefahren des Eurozentrismus möchte ich mir dies im gewählten, komplexen, interkulturellen Umfeld nicht anmaßen, sondern übe ich mich im Rahmen einer Dissertationsschrift in Bescheidenheit. Als Wissenschaftler will, soll und muss ich zwar Verantwortung für mein Handeln übernehmen; dies beinhaltet jedoch nicht, anstelle legitimer Vertreter Politik zu betreiben.

Eine Darstellungsform, mittels derer ich Verantwortung für diese Arbeit übernehmen möchte, geschieht über die Verwendung der Ich-Perspektive. Im Rahmen partizipativer Studien bestimmt die Persönlichkeit des Autors die Erkenntnisse entscheidend mit (Hauser-Schäublin 2003: 50f.; Reithofer 2009: 360). Diese persönliche Note möchte ich nicht kaschieren, sondern über die Verwendung der Ich-Perspektive unterstreichen (Kretzenbacher 1995: 27-34; Wolfsberger 2010: 112-15). 


\section{A Methodologie}

In diesem Abschnitt werden nicht nur die einzelnen Methoden in einem geschlossenen Design partizipativer Methodik vorgestellt. Darüber hinaus werden explizit die dahinterliegenden methodologischen Forschungsprinzipien und Wissenschaftsperspektiven erläutert. Auf dieser Meta-Ebene können die ethischen Zielsetzungen der Forschungspraxis dargestellt werden. Da sowohl die forschungsethischen wie entwicklungspraktischen methodologischen Perspektiven partizipativer Forschung in der humangeographischen Literatur bisher wenig beleuchtet sind und partizipative Methoden allgemein noch nicht ganz im wissenschaftlichen Mainstream angekommen sind, wird die in dieser Arbeit verwendete Methodik besonders gründlich vorgestellt.

Das erste Kapitel zeigt einen praktischen Entwurf, wie partizipative Methoden für den fidschianischen, küstendörflichen Kontext so zusammengestellt wurden, dass sie sowohl ihren wissenschaftlichen, humangeographischen, als auch ethischen und entwicklungspraktischen Ansprüchen genügen sollen. Es versteht sich jedoch keineswegs als allgemeine Blaupause für partizipative Forschung. Die Offenlegung nicht nur des Forschungsprozesses liegt mir sehr am Herzen, sondern sofern für diese Studie von Relevanz - auch Einblicke in meine Persönlichkeit. So sollen die Leser dieses Werk möglichst umfassend und kritisch hinterfragen können, damit ein mehr an Wissen geschaffen werden kann.

Eine isolierte Behandlung der vier Abschnitte Methodologie und Theorie, kulturräumlicher Überblick und Empirie ist weder möglich noch erwünscht, da sie sich gegenseitig stimulieren. Inhaltlich werden daher Aspekte entwicklungstheoretischer Konzepte aufgrund inhaltlicher Interdependenzen und um Redundanzen zu vermeiden bereits in diesem Kapitel vorgezogen behandelt. 



\section{Partizipation als Forschungsprinzip in interkulturellen Kontexten}

Auf Grundlage ethischer Vorüberlegungen zum Forschungsverhalten in interkulturellen Kontexten wird in diesem Kapitel der Partizipationsbegriff vorgestellt. Die Entstehung partizipativer Methoden wird normativ erläutert und ihre Anwendung im Feld begründet. Anschließend werden die angewandten Methoden im Hinblick auf die Forschungsziele erläutert. Zuletzt wird die Methodik an ihrem eigenen Anspruch kritisch gemessen.

Die gewählte Methodik ist im Wesentlichen von Erkenntnissen partizipativer Forschung und von methodologischen Überlegungen der Ethnographie inspiriert. Diese Dissertation bedient damit Forderungen nach mehr Ethnographie in der Humangeographie, sowie speziell in der geographischen Entwicklungsforschung (Müller 2012: 179f.; Rothfuß 2012; Verne 2012). Im Gegensatz zu klassischen qualitativen oder quantitativen Forschungen existierten zu Beginn der Erhebungsphase weder eine fokussierte Forschungsfrage, die es zu beantworten galt, noch eine Hypothese, die es zu überprüfen galt, nicht einmal das Thema konnte genauer konkretisiert werden. Aufgrund des interkulturellen Kontextes wurde der Rahmen mittels Partizipation gemeinsam mit den Dorfbewohnern erarbeitet.

\subsection{Relevanz des Forschungsthemas, Auswahl der Methodik und des Untersuchungsgebietes}

Drei zentrale, logisch miteinander verknüpfte Anliegen zur Beurteilung des Sinns humangeographischer Forschung sind für gewöhnlich Fragen nach:

- der Bedeutung möglicher Erkenntnisgewinne des Themas für Wissenschaft und Praxis;

- der Sinnhaftigkeit des gewählten Untersuchungsstandortes zur Erreichung des Ziels;

- der Angepasstheit der hierfür gewählten Methodik auf das Ziel und den Standort.

Aufgrund der besonderen methodischen Herangehensweise dieser Arbeit können sie nur ungewöhnlich, weil einer anderen logischen Verknüpfung folgend, beantwortet werden.

Im Zuge meiner Forschung zur Magisterarbeit war ich vier Monate auf den Fidschi-Inseln und habe mithilfe des Methodenpakets participatory rural appraisal (PRA) in einem Küstendorf auf Gau Island geforscht (Fink 2010b). Daher rühren ein methodisches und kulturelles Vorverständnis, Empathie, Interesse und somit die Forschungsmotivation. Die methodische Vorüberlegung dieser Studie, das Forschungsthema gemeinsam mit den Dorfbewohnern partizipativ zu erschließen, garantiert ein Thema von hoher Relevanz für die örtliche Bevölkerung. Es interes- 
siert die Menschen vor Ort, ein gutes Leben zu führen, diesbezügliche Herausforderungen zu orten und Wege zu erkunden, wie diese bestmöglich angegangen werden können. Daher ist genau dies das Thema der Arbeit und verspricht wissenschaftliche wie praktische Relevanz. Die menschzentrierte und anwendungsorientierte - damit sinnhafte, Nutzen erzeugende - Forschung dieser Arbeit stimmt entsprechend mit Kernzielen humangeographischer bzw. gesellschaftswissenschaftlicher Arbeiten allgemein überein. Wissenschaftstheoretisch findet hier allerdings eine Umkehr statt, dass zunächst der soziale Raum und die Methodik vorkonzipiert wurden, und erst im nächsten Schritt das Thema erarbeitet wurde bzw. die Erarbeitung des Themas eine zentrale empirische Leistung darstellt.

\subsection{Ethik in der interkulturellen Forschung}

Ethische Aspekte werden in geographischen Lehrbüchern oder solchen der sozialwissenschaftlichen Datenerhebung mitunter gar nicht (Gebhardt et al. 2011a; Mattissek et al. 2013; Mayring 2002), oftmals nur allenfalls sporadisch oder als Randphänomen angesprochen und gerne getrennt von der eigentlichen Qualität bzw. Güte einer Erhebung betrachtet. Ihnen wird mitunter ein Gegensatz attestiert, dass höchste wissenschaftliche Güte ethische Dilemmata hervorbringen könnte (Heidemann 2011: 40-42). Diese Arbeit hingegen stellt ein Plädoyer dar, ethische Aspekte als elementaren Bestandteil der Qualität einer Forschung aufzufassen: Im Kontext interkultureller Forschung dieser Arbeit zeigt sich, dass die Einhaltung ethischer Grenzen zur qualitativen Aufwertung des Erkenntnisgewinns führt und umgekehrt ethisch verwerfliche Forschung qualitativ minderwertig sein kann. Dieses Kapitel soll Orientierung geben, Ethik und Wissenschaftlichkeit in der Forschung vereinen zu können. Ethik und Moral werden dabei in dieser Arbeit nicht als definierbare Endzustände verstanden. Moralisches Handeln kann nicht anhand eines finiten Ideals bewertet werden. Jedoch können komparativ Forschungsverhalten verglichen werden und Verbesserungen erzielt werden.

Zur Gewährung eines hinreichenden Einblicks in die Lebenswelt der Menschen, deren Interessen diese Arbeit zu vertreten gedenkt, durfte es zur Vorbeugung eines Eurozentrismus keine Anmaßung über die Relevanz der erörterten Themen meinerseits geben. Stattdessen ist es das Anliegen der Studie, auch vordergründig nicht-wissenschaftliche Beiträge nicht abzuqualifizieren, sondern über einen integrativen Ansatz in die Welt der Wissenschaft einzubetten. Demzufolge ist der Anspruch dieser Studie, einen Beitrag dazu zu leisten, die interkulturelle humangeographische Forschung um partizipative methodologische Denkweisen sowie transdisziplinäre, integrative Konzepte zu bereichern. Darüber hinaus arbeitet die Dissertation anwendungsorientiert zu einem Themenkomplex mit hoher Relevanz vor Ort, um einen praktischen Nutzen der gewonnenen Erkenntnisse für die fidschianischen Küstendorfbewohner zu generieren. Mittels einer kritischen Reflektion und Diskussion mit der Literatur enthält diese Dissertation dennoch zugleich grundlegende Erkenntnisse sowohl über methodologische For- 
schungsprinzipien als auch über Auswirkungen von Transformationsprozessen auf Lebensqualität, unabhängig der gewählten Region und Personengruppe.

\subsubsection{Vereinbarkeit von Ethik und Wissenschaft bei partizipativer Forschung}

Anleitungen zu partizipativen Methoden legen nahe, den drei methodologischen Anspruchsdimensionen dieser Studie nach wissenschaftlicher Güte, entwicklungspraktischer Anwendungsorientierung und ethischer Qualität könne man mittels partizipativer Forschung gerecht werden (Kumar 2002; Neubert 2001; Neubert et al. 2008). Zunächst werde ich die drei Postulate genauer vorstellen, auf deren Grundlage mein Forschungsprozess startete. Im weiteren Verlauf dieses Kapitels werden die Annahmen kritisch hinterfragt und zum Teil widerlegt. Entsprechend habe ich meine Methodik im Forschungsverlauf angepasst, um den ethischen Ansprüchen besser zu genügen. Zugleich soll diese Darstellungsform der Bedeutung der Flexibilität partizipativer und ethnographischer Forschung gerecht werden.

Erstens könne bereits während des Forschungsprozess ein Nutzen für die Partizipanten gewährleistet werden. Denn besonders verwundbare Menschen stehen in Mittelpunkt einer partizipativen Studie. Die Teilnehmer führen bei partizipativen Methoden den Großteil der Interpretation und Analyse der Daten eigenständig durch, übernehmen Eigentümerschaft ${ }^{6}$. Sie entwickeln nicht bloß einen Bezug zur Studie, sondern identifizieren sich mit dieser und übernehmen Verantwortung. Sie bestimmen die für sie wichtigen Themen, sodass eine höchstmögliche Relevanz der Forschung für die Partizipanten gewährleistet werden kann. Dabei agieren sie gemeinschaftlich in Gruppendiskussionen, womit Verzerrungen durch Einzelmeinungen minimiert werden. Sie reflektieren gemeinsam über die relevanten Themen und sind an der Qualität der Ergebnisse interessiert, investieren Zeit und Muße in die Studie, da ein Nutzen erkennbar und erwartbar ist, sodass bereits in der Erhebungsphase empowerment ${ }^{7}$ gesteigert wird. Damit hat alleine schon der partizipative Forschungsprozess unabhängig der aus ihm gewonnenen Daten einen entwicklungspolitischen Nutzen. Nicht nur haben die Ergebnisse der Studie einen Anwendungsbezug, sondern die Erhebungsphase selbst stellt einen entwicklungspraktischen Beitrag dar.

6 Ownership steht hier bewusst entgegen der landläufigen Übersetzung der Entwicklungszusammenarbeit nicht für Eigenverantwortung (Nuscheler 2008: 14-16), sondern für Eigentümerschaft. Ownership beinhaltet den Gedanken, etwas sein Eigen zu nennen, zu besitzen. Besitztümer möchte man in der Regel bewahren, pflegen und gegebenenfalls vermehren, da man sich einen Nutzen verspricht. Dieser positive Anreiz kommt nicht deutlich genug zum Vorschein, wenn man lediglich die aus dem Besitz indirekt resultierende Komponente der Verantwortung in den Mittelpunkt stellt, welche Pflichten anstelle des Nutzens suggeriert.

7 Der englische Begriff steht für die Stärkung von Macht und Einfluss lokaler Akteure, für einen autogenen Prozess des Zugangs zu Mitwirkungs- und Beteiligungsmöglichkeiten einer Person, der sie zur aktiven Gestaltung des eigenen Lebens sowie der Gemeinschaft befähigt (Holtz 2011: 45, Willis 2005:102f.). 
Zweitens würden partizipative Studien neue Einsichten für Außenstehende (wie mich oder - so der Anspruch - die Leser dieser Studie) in die Lebensrealität und den damit verbundenen Werteverständnissen und Prioritätensetzungen der Menschen gewähren. Hierfür übt sich der Forscher bei der Steuerung der Diskussion in Zurückhaltung, lenkt diese optimalerweise gar nicht, sondern nimmt allenfalls vermittelnde Positionen ein. Somit agieren die Teilnehmer möglichst unverzerrt, bearbeiten Themen eigenständig und setzen nach ihrem Ermessen Schwerpunkte. Diese Offenheit gewähre ungeahnte Einblicke in das sozio-kulturelle Setting der Studie und steigere das Verstehen der Ergebnisse der Analysen auf Seiten der Außenstehenden.

Drittens würden Offenheit, Eigentümerschaft und Verantwortung die wissenschaftliche Qualität der Studie heben. Denn im Verlauf dieser Studie sollen Risiken erkannt und Handlungsmöglichkeiten aufgezeigt werden, die zu Wohlbefinden und Lebensqualität beitragen, sodass die Teilnehmer Wert auf ein klares Verständnis des Forschers bezüglich ihrer Lebenssituation legen würden. Da der Forschungsprozess für die Teilnehmer hochrelevant sei, sie ihren Ausgang jedoch nicht kennen, seien sie sehr an einem ausgewogenen und ausgereiften, umfassenden Einblick interessiert, um den Nutzen der Studie bestmöglich zu gewährleisten. Mittels Triangulation ${ }^{8}$ würden alle Erkenntnisse vielseitig abgesichert. Es sei daher davon auszugehen, dass die vorgetragenen Ergebnisse eine hohe Validität haben.

\subsubsection{Forschung als persönlicher Prozess mit kritischer Selbstreflektion}

Kritische Selbstreflektion sowie das gemeinsame Reflektieren mit Vertrauenspersonen des kulturell fremden Kontextes sind geeignete Wege, ethisch fragwürdiges Vorgehen in Forschungssituationen frühzeitig zu realisieren und ihnen entgegen zu steuern (Hauser-Schäublin 2003: 50f.; Reithofer 2009: 358; Steinebach 2009: 373). Dieser methodologische Abschnitt reflektiert kritisch über die eigene Vorgehensweise um auf eigenes Fehlverhalten aufmerksam zu machen. Ich möchte den Lesern meine Intentionen, aber auch Fallstricke und den persönlichen Umgang mit diesen offenlegen. Da man insbesondere aus Fehlern lernt, möchte ich die Leserschaft an meinen Fehlern teilhaben lassen.

Die angewandte Methodik ist von prozessualem Charakter. Die Forschung ist nicht standardisiert, sondern zwischenmenschlich entstanden und damit von mir als Individuum und meiner Persönlichkeit geprägt. Im Laufe der Jahre der Feldforschung setzte eine Verbesserung in der Durchführung ein. Eine identische Wiederholung ist nicht möglich. Auf einer Metaebene verdeutlicht dies für partizipative Forschung: Vollständige methodische Vorkonzeptionen sind nicht möglich, Spontanität und Flexibilität sind zwingend erforderlich, um angepasst auf die lokalen Bedingungen zu agieren. Eine „perfekte“ Vorbereitung auf das lokalspezifisch

\footnotetext{
${ }^{8}$ Triangulation - vereinfacht: Gegenprüfung - meint einerseits die Anwendung verschiedener Methoden zu gleichen Themenstellungen, andererseits die Verwendung gleicher Methoden auf verschiedene Fokusgruppen und Individuen.
} 
einzigartige Untersuchungsdorf in dem Sinne, dass Irrtümer, ethisches Fehlverhalten und Fehlinterpretationen im Vorhinein ausgeschlossen seien, ist unmöglich. Dies würde einen anfänglichen Erkenntnisstand voraussetzen, der die eigentliche Forschung obsolet machen würde.

Nachvollziehbarkeit wird in dieser Arbeit als ein Grundpfeiler wissenschaftlichen Arbeitens verstanden (Flick 2007: 511f., 522; Steinke 2007: 324); ohne sie ist keine Wissenschaftlichkeit gewährt und gewonnene Erkenntnisse wären belanglos und die Arbeit insgesamt unbrauchbar. Umgekehrt kann Nachvollziehbarkeit allein jedoch kein ausreichendes Qualitätsmerkmal sein, sodass in diesem Abschnitt weitere Merkmale vorgestellt werden. Vorderstes Anliegen bleibt dabei eine möglichst lückenlose Offenlegung der methodischen Herangehensweise, was meiner Auffassung nach Vorgehensweisen, die sich im Nachhinein als nicht zielführend oder fehlerhaft erwiesen haben, nicht verschweigt oder beschönigt.

\subsection{Partizipation in der Forschung und Entwicklungspraxis}

Im Kontext dieser Arbeit bedeutet Partizipation die Teilhabe am öffentlichen Leben. In Form einer Beteiligung an Entscheidungsprozessen hat diese Teilhabe politische Dimensionen. Als Auslebung der eigenen Kultur verstanden umfasst Partizipation zugleich sozio-kulturelle Dimensionen. Die Teilhabe an Wirtschaftsaktivitäten schließt sozio-ökonomische Dimensionen ein. Partizipation beinhaltet sämtliche Sphären der Gesellschaft. Forschungsmethodisch verstanden umfasst Partizipation wiederum die Teilhabe des Forschers am gesellschaftlichen Leben der Menschen seines Forschungsumfeldes, sowie auch umgekehrt deren Mitgestaltung der Forschung. Teilnehmende Beobachtung, welche zum Teil synonym zu Ethnographie verstanden wird, ist die klassische Methode, bei der der Forschende am Alltagsleben der Untersuchungsgemeinde teilnimmt (Spradley 1979; Spradley 1980). Seit den 1970ern werden zudem unter dem Schlagwort „Partizipation“ als Entwicklungsideal in der EZ und ihr angrenzenden, zuarbeitenden Wissenschaften gruppenbasierte Methoden entwickelt, die die Teilnahme der Untersuchten am Forschungsprozess fördern (Kumar 2002: 29-34).

Prinzipiell gibt es höchst unterschiedliche Grade, wie aktiv die Teilhabe ausfällt, welche alle unter dem Partizipationsbegriff zusammenfallen können. Wie im theoretisch-konzeptionellen Rahmen genauer dargelegt wird, sind in dieser Studie Freiheit zur Selbstbestimmung und das Recht auf Partizipation hohe Werte, sodass Partizipation normativ positiv besetzt ist und eine aktive Mitgestaltung der Gesellschaft im Sinn hat. Das Höchstmaß an Partizipation stellt die SelbstMobilisierung von Gruppen anhand kollektiver - von allen Beteiligten akzeptierten und mitgetragenen - Entscheidungen und Handlungen unabhängig externer Institutionen dar. Niedrige Level hingegen können als passive Partizipation beschrieben werden. Menschen werden hierbei lediglich in Kenntnis gesetzt, was gesellschaftlich passieren wird oder bereits passiert ist (Kumar 2002: 24f.; Spradley 1980: 58-62). Als gar manipulativ zu bezeichnende Partizipation täuscht Mitgestal- 
tung vor, obwohl die politischen Entscheidungen unabhängig der Meinungen der Betroffenen gefällt werden. Im entwicklungspolitischen Kontext werden solche Formen als neue Tyrannei der EZ gesehen (Cooke \& Kothari 2004: 3f., 7f.), welche es aus normativer Sicht in jedem Falle zu vermeiden gilt.

Die EZ war lange Zeit und ist zum Teil bis heute von Machtasymmetrien zwischen den beteiligten Entwicklungsakteuren gekennzeichnet, was einen Eurozentrismus begünstigt. Dies äußert sich vor allem dann, wenn die Deutungshoheit, was Entwicklung sei, ausländischen Hilfsorganisationen obliegt, und diese maßgeblich den Maßnahmenkatalog zusammenstellen, wie diese Entwicklung im Zielort voranzutreiben sei. Um der Asymmetrie entgegen zu wirken, wird in der bi- und multilateralen staatlichen EZ der top-down Ansatz zunehmend um einen bottomup Ansatz ergänzt. Insbesondere NGOs befürworten Graswurzel-Bewegungen, also reine bottom-up Ansätze, in denen die lokale Bevölkerung ihre Probleme eigenständig definieren soll und deren Lösungen selbst bestimmt. Beiderseits findet die Bedeutung einer weitreichenden Partizipation der Ortsansässigen für eine erfolgreiche EZ wachsende Akzeptanz (Ebeling et al. 2007: 337; Nuscheler 2008: 12; Thiel 2001: 29).

Üblicherweise werden Sozialforschungsmethoden nach qualitativen und quantitativen unterschieden. Qualitative Sozialforschung zielt demnach auf das Verstehen gesellschaftlicher Phänomene ab. Ihr Interesse ist die Qualität des betrachteten Forschungsgegenstandes. Ihre induktive Herangehensweise wird häufig zur Bildung von Theorien oder Hypothesen herangezogen. Im Gegensatz hierzu zielt quantitative Sozialforschung auf die Überprüfung von Hypothesen und Theorien. In ihrer deduktiven Herangehensweise versucht sie Gesetzmäßigkeiten zu erkennen. Mittels quantitativer Angaben wie Mittelwerten, Verteilungen oder Wahrscheinlichkeiten sollen Gegenstände nicht nur beschrieben, sondern auch erklärt werden und Zusammenhänge erläutert werden (Meier Kruker \& Rauh 2005: 3-5). Das hiesige Verständnis von Partizipation stellt einen dritten methodologischen Weg dar, welcher die ethischen und wissenschaftlichen Schwächen beider Lager zu überwinden sucht, die bei ihrer Anwendung im interkulturellen Kontext auftreten.

Stellt man sich die Frage, wer Wissen schafft und für wen dieses Wissen geschaffen wird, so nimmt die Wissenschaftscommunity selbst eine prominente Stellung ein. Auch wenn die vorliegende Arbeit sich als Dissertationsschrift wieder an genau diese Wissenschaftswelt richtet, so versuchte die vorangegangene Forschung doch, diese Strukturen zu überwinden und hebt die untersuchte Gesellschaft selbst in die Rolle des Forschers und des primären Adressaten. So bin ich Initiator der Forschung, jedoch nicht alleiniger Forscher oder gar Experte. Den Großteil der Forschung übernehmen die Dorfbewohner und sie besitzen die Deutungshoheit.

Mir obliegen stattdessen in erster Linie die Initiierung und Koordination der Forschung, sowie die Zusammentragung und - nach Rücksprache und Revision die Verbreitung der Erkenntnisse. Die Aufbereitung der Forschung als vorliegen- 
de Dissertation konnte daher nicht im Zentrum des Forschungsinteresses oder dessen methodischer Konzeption gestanden haben, läuft dem Forschungsziel jedoch auch nicht entgegen. Es stehen die Belange der an der Forschung partizipierenden Gemeinden im Zentrum der Forschung und dieses Kapitel gibt Auskunft, wie dies mittels Partizipation erreicht wird. Um ihre Interessen in dieser Arbeit vertreten zu können, ist methodisch die Kommunikation zwischen den Forschern von entscheidender Bedeutung, bei der ich als Mediator fungierte.

\subsubsection{Intersubjektivität im interkulturellen Kontext}

Über Sozialisation erlernen wir die Zeichen- und Symbolsprache zu deuten, die die eigene Kultur ausmacht um uns so in dieser zu bewegen. Abhängig vom Kontext und über die Zeit können Handlungen und Symbole unterschiedliche Bedeutungen haben. Im interkulturellen Kontext gilt es, die komplex überlagerten oder ineinander verwobenen Deutungsvarianzen zu entschlüsseln. Ethnographie ist daher eine interpretierende Wissenschaft, um gesellschaftliche Ausdrucksformen in ihrem kulturellen Kontext zu erläutern (Geertz 1983: 9-15; Heidemann 2011: 20-25).

Mit der eigenen Interpretation geht - trotz eines Abgleichens mit bereits existierender ethnographischer Forschung auf den Fidschi-Inseln - eine gewisse Subjektivität einher. Dank der Teilhabe am öffentlichen Leben in fidschianischen Küstendörfern konnte ich gemeinsam Erfahrungen erleben, nahm diese jedoch individuell wahr und interpretiere diese subjektiv vor meinem eigenen Werte- und Erfahrungshorizont, welcher sowohl vom eigenen kulturellen Hintergrund als auch den Erlebnissen vor Ort beeinflusst ist. Darüber hinaus konnten die Dorfbewohner ihre Eigeninterpretationen mittels intensiver Kommunikation intersubjektiv für mich nachvollziehbar machen. Gleichfalls strebe ich nun an, diese Erkenntnisse für die Leserschaft intersubjektiv nachvollziehbar aufzubereiten. Objektivität wird also durch intersubjektive Nachvollziehbarkeit als wissenschaftliches Qualitätskriterium ersetzt. Ich möchte mich damit im Schatten Hannah Arendts bewegen, die Intersubjektivismus als Grundlage ihrer Forschung ansah, um der menschlichen Pluralität gerecht zu werden. Mittels intersubjektivem Nachempfinden positioniert sie sich als teilnehmende Denkerin anstelle einer distanzierten Wissenschaftlerin (Heuer 2006: 8-10). In der Ethnologie gehören Teilnahme und Intersubjektivität zu ethischen wie wissenschaftlichen Standards der interkulturellen Forschung (Heidemann 2011: 16f., 36-38).

\subsubsection{Verzerrungen entwicklungspolitischer Forschung}

Das dieser Arbeit zugrundeliegende Verständnis von Partizipation ist im Wesentlichen der praktischen EZ entsprungen. Partizipation bildet eine Antwort auf vielfältige Wahrnehmungsverzerrungen, denen die zumeist westlichen Entwicklungsakteure im Kontext kultureller Fremde leicht unterliegen. Ohne ein hohes $\mathrm{Maß}$ an Partizipation sind konzeptionelle Fehler wahrscheinlich, welche die zu- 
meist guten Absichten konterkarieren. In Anlehnung an Somesh Kumar (2002: 34-38) möchte ich mehrere Problemfelder, aus denen Verzerrungen bzw. Befangenheiten traditioneller „Entwicklungsexperten“ resultieren können, kurz vorstellen:

- Expertenhierarchie: Im Sinne der Kritik am Eurozentrismus gehen Experten oftmals „davon aus, dass es im Süden gesellschaftliche Probleme gibt, für die im Norden Problemlösungskompetenz vorhanden ist - nicht aber umgekehrt. [...] [Dieser Denkweise zufolge verfügen Experten] über privilegiertes Wissen über die Defizite der Lebensweisen Anderer“ (Müller \& Ziai 2015: 9). Als Experte beispielsweise für Gesundheitsaspekte rückt man ggfs. eigene Spezialgebiete in den Fokus und maßt sich Urteile unabhängig der Wahrnehmung der eigentlichen Zielgruppe an, was die örtlichen Bedürfnisse nicht angemessen widerspiegelt.

- Ortszugänglichkeit: Aufgrund von Logistik, Zeit- und Kosteneffizienz besteht ein Hang, gut erreichbare Forschungsstandorte zu wählen, welche die Problemkonstellationen im für die Studie beanspruchten Gesamtuntersuchungsgebiet nicht repräsentativ abdecken. Die Verwundbarsten - und damit zumeist die eigentlichen Hauptzielgruppen - bleiben möglicherweise außen vor.

- Arbeitszeiten: Finden Forschungen zu festen Arbeitszeiten statt, beispielsweise tagsüber, sind womöglich bestimmte Personengruppen nicht ansprechbar oder gar nicht erst wahrnehmbar und essentielle Herausforderungen der Menschen, die zu anderen Tageszeiten auftreten, können übersehen oder fehlinterpretiert werden.

- Saisonalität: Bestimmte Herausforderungen wie beispielsweise wetterbedingte Naturgefahren, aber auch Arbeitsmarktnachfrageschwankungen, treten oft saisonal auf und könnten bei kurzzeitigen Forschungsaufenthalten falsch bewertet werden.

- Persönlichkeit des Forschers: Persönliche Merkmale des Forschers können eine Reihe von Verzerrungen im Umgang mit der lokalen Bevölkerung hervorrufen:

o Geschlecht: Forscher stützen sich auf Gespräche mit Ortsvertretern des eigenen Geschlechts, ggfs. aufgrund gesellschaftlicher Vorbehalte gegenüber einer Interaktion beispielsweise zwischen einem fremdem, männlichem Forscher und Dorfbewohnerinnen, weshalb in einem solchen Fall die Bedürfnisse von Frauen untergehen können oder fehlinterpretiert werden.

o Sprache: Insbesondere wenn der Forscher der Landessprache oder dem örtlichen Dialekt nicht mächtig ist, stützt er sich leicht auf diejenigen, die des Englischen mächtig sind, deren individuelle Themen jedoch nicht zwingend die Gesamtheit abdecken.

o Örtliche Elite: Aufgrund möglicher Zugangsschwierigkeiten sprachlicher, kultureller oder zeitlicher Natur mag der Forscher lieber mit der 
Bildungselite vor Ort kommunizieren, da diese leichter verständlich erscheint. Jedoch vermag die Elite die Bedürfnisse der Verwundbarsten als wesentliche Adressaten der Studie ggfs. nicht adäquat wiederzugeben.

- Diplomatie: Themengebiete, die vom Forscher als sensibel empfunden werden, oder die er als von den Bewohnern als sensibel empfunden wahrnimmt, bleiben ggfs. unberücksichtigt. Umgekehrt möchten die Ortsansässigen den fremden Forscher nicht zwingend mit ihren persönlichen Problemen belasten. Auch auf spezifische Lebenssituationen von Menschen mit Behinderungen und deren Angehörigen wird nicht zwingend eingegangen.

- Projektbezogenheit: Insbesondere bei der Forschung in bereits laufenden Entwicklungsprojekten wird der außenstehende Forscher gerne zunächst mit den laufenden, als entwicklungsfördernd propagierten Maßnahmen konfrontiert, sodass er vorbelastet ins Feld zieht, die lokale Situation einseitig wahrnimmt und kein Korrektiv möglich ist. Dem Projekt nicht Wohlgesinnte verwehren ihm zudem möglicherweise entscheidende Auskünfte.

\subsubsection{Minimierung von Verzerrungen mittels Partizipation}

Mittels Partizipation können die genannten entwicklungspraktischen Verzerrungen reduziert werden, so konnten auch in der hiesigen Forschung viele Formen der Verzerrung im Vorfeld vermindert werden. Der erste von drei Forschungsaufenthalten erfolgte im Rahmen meiner Magisterarbeit „Verwundbarkeit ländlicher Küstenbewohner auf den Fidschi Inseln gegenüber Naturgefahren. Eine partizipative livelihood-Analyse" (Fink 2010b). Hier war ich im Forschungsprojekt „Vulnerability Mapping as a Policy Tool in Developing Countries“ des AsiaPacific Network for Global Change Research (APN) eingebunden (Fink 2010a). ${ }^{9}$ Die weitere Forschung zur vorliegenden Dissertationsschrift unterlag keiner externen Bindung, sodass das Problem der Projektbezogenheit aufgehoben ist. Dafür unterlag sie jedoch finanziellen Limitationen.

Aus Zeit- und Kosteneffizienzgründen sind in den drei Studienaufenthalten von insgesamt knapp 14 Monaten die Monate April bis Juli nicht abgedeckt und die entlegensten Inseln der Lau Gruppe wurden nicht persönlich aufgesucht, sodass Verzerrungen hinsichtlich Ortszugänglichkeit und Saisonalität nicht vollständig aufgehoben sind. Die zentralen Aufenthalte in den drei Dörfern sind bewusst

\footnotetext{
9 Trotz vieler Freiheiten war damit der Themenbereich der Forschung eingegrenzt, sodass kein Höchstmaß an Partizipation gegeben war. Umgekehrt wurde mir darüber erst der Zugang zur dörflichen Lebenswelt sowie in ein Netz von Experten ermöglicht und ich erhielt materielle wie immaterielle Unterstützung, für die ich sehr dankbar bin. Im Rahmen dieser ersten Studie reifte zudem der Gedanke, dass nicht Untersuchungen, die die Überwindung einer Verwundbarkeit gegenüber Naturgefahren ins Zentrum des Interesses setzen, sondern solche, die das sozio-kulturelle Miteinander in den Fokus rücken, entscheidendere Erkenntnisse zur Steigerung der Lebensqualität der Dorfbewohner liefern können.
} 
auf die Weihnachtszeit gelegt worden, sodass - da nicht jedes Dorf ein ganzes Jahr begleitet wurde - zumindest eine saisonale Einheitlichkeit der Untersuchungszeiten in den Vergleichsdörfern gewährleistet werden kann. Da es sich dabei um kleine Dörfer von unter 150 Einwohnern und jeweils fünfwöchige Daueraufenthalte in den Hauptuntersuchungsdörfern handelte, konnte jeder Dorfbewohner wahrgenommen werden. Probleme einhergehend mit festen Arbeitszeiten und örtlichen Eliten konnten so minimiert werden.

Wesensmerkmale partizipativer Forschung sind Offenheit in der Forschung, Zurückhaltung im Forschungsprozess und beständige kritische Selbstreflektion. Diese Themen werden in diesem Kapitel ausführlich behandelt um aufzuzeigen, wie darüber die vermeintliche eigene Expertise nicht zum Problem wird. Ein entscheidender Vorteil partizipativer Forschung ist zudem der Aufbau von Vertrauen während des Forschungsprozesses, wie in diesem Kapitel ebenfalls ausführlich erläutert wird. So wurden mir manche sensible Themen bewusst anvertraut, ohne dies forcieren zu müssen und damit ethische Dilemmata zu schaffen, sodass Verzerrungen durch Diplomatie reduziert sind.

\subsubsection{Umgang mit Verzerrungen aufgrund der eigenen Persönlicbkeit}

Manche persönliche Charakteristika wie Alter und Geschlecht eines Forschers sind nicht ausschaltbar. Über die Hinzunahme von mit der Kultur vertrauten Forschungsassistenten und einer partizipativen Arbeit in gemischtgeschlechtlichen Teams konnten Verzerrungen jedoch reduziert werden (Keck 2003: 207-209). Insbesondere Möglichkeiten offener und ehrlicher Kommunikation zwischen Forschern und Assistenten zur kritischen Reflektion des Erfahrenen halfen hierbei der Minimierung der Verzerrungen. Ich besitze nur Grundkenntnisse im Fidschianischen, was die Qualität der Studie beeinträchtigt (Beuselinck 2000: 88; Pole 2000: 106; Senft 2003: 55-61). In den Untersuchungsdörfern war jedoch jeder Jugendliche und Erwachsene des Englischen mächtig. Um niemanden aufgrund von Sprachschwierigkeiten auszuschließen, wurden entscheidende Methoden auf Fidschianisch abgehalten, wobei die Forschungsassistenten als Übersetzer fungierten. Zitate in dieser Arbeit unterliegen daher teils einer doppelten Übersetzung, bei welcher nicht zwingend der genaue Wortlaut wiedergegeben werden kann, wohl aber wurde sich um die inhaltlich korrekte Wiedergabe bemüht. Eine gänzliche Ausschließung persönlicher Merkmale auf das Ergebnis der Forschung scheint nicht möglich. Aufgrund ethischer Überlegungen ist dies jedoch auch nicht wünschenswert, da der Forscher seine Persönlichkeit nicht kaschieren sollte. Ein offener Umgang mit persönlichen Stärken und Schwächen steigert die Glaubwürdigkeit, schafft Vertrauen und festigt damit die Basis valider Forschungsergebnisse.

Für diese Arbeit wurde eine enge Zusammenarbeit aller Beteiligten angestrebt. Als Initiator, Koordinator und Mediator kommt mir dabei eine Schlüsselrolle zu. Der eigene Einfluss auf die Forschung bleibt zu jeder Zeit omnipräsent. Die Be- 
deutung der eigenen Persönlichkeit auf die hiesige Studie ist nicht zu unterschätzen. ${ }^{10} \mathrm{Da}$ ein gemeinsames Forschen mit den Menschen vor Ort erreicht wurde, unterlag die eigene Interpretation jedoch einer permanenten korrektiven Kontrolle.

Im Ergebnis steht insgesamt eine Studie, die sich im Bereich der Humangeographie einordnen lässt. Dies unterstreicht die Bedeutung der eigenen Person. Diese Erkenntnis schmälert nicht die Relevanz des Themas für die Ortsansässigenen, da es zusammen mit ihnen erarbeitet wurde. Lediglich kann und möchte sich diese Studie nicht anmaßen, sich dem einzig bedeutungsvollen Thema zu widmen, oder den Superlativ zu gebrauchen, das relevanteste Thema mit den und für die Menschen vor Ort ausfindig gemacht zu haben.

\subsection{Macht und Vertrauen in partizipativer Forschung}

Im Zuge der Vorarbeit im Rahmen der Magisterarbeit konnte ich feststellen, dass die partizipative Herangehensweise vielversprechend ist, da ich in dieser Zeit bereits eine Vertrauensbasis aufbauen konnte. Ohne ein solches gegenseitiges Vertrauen wären mir die Wünsche, Hoffnungen, Ängste und Herausforderungen nicht anvertraut, sowie ein gewissenhafter Umgang mit diesen nicht zugetraut worden. Bevor es zu irgendeiner Form der Datenaufnahme kommt, benötigt es zunächst einmal eines gegenseitigen Vertrauens (Meier Kruker \& Rauh 2005: 62f.). Über Partizipation als Teilhabe am öffentlichen Leben der Mitmenschen und Integration in die Gesellschaft kann der Forscher gesellschaftliche Akzeptanz bekommen und Vertrauen gewinnen auf welcher sich die Kooperationsbasis gründet. Die partizipative Herangehensweise ermöglicht so die Generierung eines Forschungsthemenkomplexes von beträchtlicher Relevanz. Diese Dissertation stellt nun meinen Versuch dar, dies der Leserschaft zu vermitteln und damit das in mich gesetzte Vertrauen zu bestätigen.

Die Schaffung dieser Vertrauensbasis ist dabei nicht allzu sehr mein Verdienst, sondern liegt vor allem in den kulturellen Werten der Fidschianer und deren Auslebung begründet. Respekt, Vertrauen und Toleranz Fremden gegenüber sind weitverbreitete Ideale (Ravuvu 1987: 18f.), wie auch im ersten Exkurs deutlich wurde. Eine mögliche Erklärung kann in der Abgeschiedenheit der Inselgruppen liegen, die zumindest unter vergangenen Transportbedingungen kulturellen Austausch erschwerte und die seltenen Gelegenheiten hierzu zu einem hohen Gut machten. Dies setzt die Erkenntnis voraus, dass über den Austausch Informatio-

\footnotetext{
10 Aufgrund von Persönlichkeitsmerkmalen war der Kontakt zu manchen sozialen Gruppen intensiver als zu anderen. So konnte ich generell mit der männlichen Jugend losgelöster sprechen und am besten sensible Probleme diskutieren. In Gruppendiskussionen wurde ansonsten oft ausweichend auf sensible Themen reagiert. In Einzelgesprächen erhielt ich zwar weitere Einblicke, diese sind jedoch eher individuell geprägt denn auf Gruppen verallgemeinerbar und mitunter nicht für eine Veröffentlichung bestimmt. Aus Datenschutzgründen erhalten sie daher nur geringfügigen Eingang in diese Studie.
} 
A: Methodologie

nen fließen und Fremde damit besonders gute Möglichkeiten für Erneuerungen, Verbesserungen, Fortschritt und Entwicklung schaffen. Neben Informationen fließen nach fidschianischem Brauch auch immer materielle Gastgeschenke, welche aufgrund der Begrenztheit lokaler Ressourcen ebenfalls bedeutend die Lebensqualität steigern (Veitayaki 1995: 121f.). Da ich Dörfer entsprechend der Traditionen stets mit einem dem Dorf vertrauten Fürsprecher betrat und die Willkommensbräuche beachtete, fiel das Vertrauen, welches meinem Fürsprecher entgegengebracht wurde, auch auf mich ab, sodass ich kulturell bedingt die Forschungsaufenthalte jeweils mit einem enormen Vertrauensvorschuss begann.

\subsubsection{Machtpolitische Dimensionen von Partizipation}

Sowohl in der qualitativen als auch quantitativen Forschung bestehen Machtungleichgewichte zwischen Forschern und Befragten. Bei strukturierten Befragungen mit strukturierten Fragebögen als quantitative Beispielmethode kennt der Forscher bereits die Fragen, hat diese selbst konzipiert und stets den alleinigen Überblick über den Interviewprozess und die Lenkungsmacht über den Ablauf. Auch bei nicht-standardisierten Interviews als qualitative Beispielmethode ist der Forscher alleiniger Fragesteller und Wissen wird einseitig zugunsten des Experten extrahiert. ${ }^{11}$ Im interkulturellen Kontext schadet beides ungemein dem Erkenntnisgewinn. Denn als externer Experte ist man für den Untersuchten zunächst einmal ein Fremder. Übt dieser Macht aus, welche ihm seitens der Befragten nicht legitim anvertraut wurde, sorgt dies für Irritationen und Misstrauen. Mangelndes Vertrauen in den Forscher und in dessen gewissenhaftem Umgang mit den von ihm verlangten Informationen führt berechtigterweise zu Zurückhaltung, Verweigerung oder Falschaussagen. Partizipative Forschung setzt sich daher zum Ideal, Machtungleichgewichte in der Forschungspraxis zu überwinden und eine Kommunikation auf Augenhöhe anzustreben. Dies bedeutet jedoch nicht, dass partizipative Forschung keine machtpolitischen Implikationen hätte.

Partizipative Methodensets wie die PRA-Toolbox finden in einem räumlich und zeitlich begrenzten Kontext statt. In nach sozialen Merkmalen gegliederen Gruppen erarbeiten die Teilnehmer Problemanalysen und erarbeiten Lösungsvorschläge. Entwicklungspolitisch soll dies die Betroffenen empowern und Eigentümerschaft in Entwicklungsprojekten erzeugen. Die Entscheidungsprozesse finden somit abseits bereits existierender staatlicher oder gesellschaftlicher institutionalisierter Ordnungen statt. Bestehende Hierarchien werden hierbei unterwandert um

\footnotetext{
${ }^{11}$ Die eigene Persönlichkeit wurde für die Studie nicht zurückgestellt. Auf Nachfrage wurden politische Ansichten und moralische Werte nicht verschwiegen, sich wohl aber bemüht, diese im Rahmen der örtlichen ethischen Kommunikationsgebote zu präsentieren. Ich versuchte hierbei Einblicke in meine Kultur und deren Historie zu geben, um vor diesem Horizont meine Wertemaßstäbe und Ansichten nachvollziehbar zu gestalten - Überzeugungsarbeit war nicht mein Ziel, sondern das Verständnis von Pluralität zu steigern. Diese Konversationen wurden gemeinhin als spannend und lehrreich empfunden und dankbar angenommen, sodass ich hierüber der generellen Wissensextraktion seitens der Forscher ein Stück weit entgegenwirken konnte.
} 
eine „Zivilgesellschaft““ aufzubauen (Korf 2009: 99). Partizipative Forschung stellt somit in jedem Falle eine Intervention in bestehende politische Strukturen dar. Die Forschungsmethoden können in illegitime Ausübungen von Macht ausarten, weshalb Partizipation leicht zu einer neuen Tyrannei der Entwicklungszusammenarbeit werden kann (Cooke \& Kothari 2004: 3f., 7f.).

Going native, das nahezu vollständige Aufgehen des Forschers in sein Forschungsumfeld, wird gemein hin als Problem in der Forschung verstanden, da der Forscher aufgrund der Identifikation mit den Untersuchten schnell einseitig und kurzsichtig argumentieren würde, da er die Weitsicht über übergeordnete Phänomene verliere (Müller 2012: 180). Dies steht in einem scheinbaren Widerspruch zu anderen Ideen partizipativer Forschung. Denn um das benötigte Vertrauen generieren zu können, ist eine Identifikation mit dem örtlichen Umfeld fundamental. So bescheinigt diese Arbeit einem Einleben in den neuen kulturellen Kontext elementare Bedeutung. Für partizipative Forschung ist ein emotionales Einlassen auf die Menschen vor Ort zwingend; ohne Emotionalität wäre echte Teilhabe undenkbar. Kritische Selbstreflektion sowie ein Abgleich mit forschungsrelevanter Literatur stellen jedoch Strategien dar, sich die Gefahren des going native bewusst zu halten. Weiterhin sollte es gerade aufgrund der Identifikation im Interesse des Forschers sein, die gewonnenen Erkenntnisse einem möglichst breitgefächerten und einflussreichen Publikum zu präsentieren, um Verbesserungen zu erzielen. Um das Interesse der Zuhörer zu gewinnen, bedarf es plausibler und ausgewogener Argumentationen und die Erkenntnisse müssen in die übergeordneten Themen und Diskurse der Wissenschaftswelt bzw. Politik eingebettet werden, ${ }^{12}$ weshalb Einseitigkeit und Kurzsichtigkeit nicht ans Ziel führen. Eine Stellungnahme bzw. Positionsbeziehung seitens des Forschers ist in solchen Fällen nicht verwerflich oder unwissenschaftlich, sofern die Position kenntlich gemacht wird, die Argumentation jedoch ausgewogen ist und letztlich die Position im Ergebnis begründet nachvollziehbar erscheint.

\subsubsection{Qualität von Gruppenarbeiten und Visualisierungen}

Methodisch bieten sich zur Reduktion der Machtamplitude anstelle von Interviews allenfalls Gruppendiskussionen an, die vom Forscher zwar initiiert, jedoch nicht gesteuert werden. Forscher sollten maximal vermittelnde und diskussionsanregende Stellungen beziehen, um die Meinungen der Diskutanten nicht zu beeinflussen. Diese Zurückhaltung soll eine Dynamik in der Diskussion fördern und insgesamt eine große Offenheit der Forschung gewährleisten (Flick 2007: 250252), eine der größten Stärken partizipativer Forschung. Denn gerade beim Forschen unter Bedingungen kultureller Fremdheit können sich beim Forscher schnell falsche Vorstellungen über den Untersuchungsgegenstand bilden, die aufgrund der Offenheit jedoch erkannt und revidiert werden können (Mayring 2002:

${ }^{12}$ So verzichtet auch diese Dissertation selbstverständlich nicht auf eine theoretisch-konzeptionelle Rahmung. 
27f.). Schon allein über die Bildung diskussionsfähiger Gruppen wird der Gefahr entgegengewirkt, dass die Erhebung einer stereotypen Einzelmeinung unterliegt. Zur Diskussionsfähigkeit bieten sich homogene Fokusgruppen an, die soziale Merkmale teilen und für die entsprechenden sozialen Gruppen stehen. So kann die gewünschte Dynamik erzielt werden und man erhält tiefergehende Informationen, auch über sensible Themen (Kumar 2002: 43-45).

Durch die Kommunikation in Gruppen zwischen den Dorfbewohnern sollen Meinungen im Alltag geäußert und ausgetauscht werden (Flick 2007: 251). Dadurch wird das vorhandene Wissen sichtbar und kann aktiv genutzt werden. Die Gemeindemitglieder werden so selbst zu Ethnographen antrainiert. Sie helfen nicht (nur) dem Forscher, sondern entscheiden aktiv, welche Daten gesammelt werden und interpretieren diese kollektiv (McQuiston 2005: 212). Das Teilen von Ansichten, Wissen und Erfahrung stärkt Vertrauen und fördert eine Generierung potentieller Lösungsvorschläge und kollektiver Handlungen zur erfolgreichen Handhabung der bewusstgewordenen Herausforderungen. Durch das Bewusstwerden alltäglicher Phänomene soll so empowerment stattfinden. Während des Forschungsprozesses sollen damit bereits Aktiva für die lokale Bevölkerung generiert werden, indem die Diskutanten anhand der Aufdeckung ihrer eigenen Stärken ihre Handlungspotentiale besser entfalten. Partizipative Methoden verwirklichen damit Leitgedanken der Entwicklungspolitik bereits in der Forschung, was eine Unterscheidung zwischen methodischen Prinzipien und entwicklungspolitischen Zielen erschwert (Neubert et al. 2008: 97f.) bzw. erübrigt.

Die Anwendung partizipativer anstelle qualitativer oder quantitativer Methoden liegt daher nicht einzig in der Güte der jeweiligen Methodik zur Datengewinnung begründet. ${ }^{13}$ Die Verschiebung der Forschungsmethoden erklärt sich stattdessen anhand der veränderten forschungsethischen und entwicklungspolitischen Zielsetzung. Nicht die Güte einer Methodik selbst, sondern ihr Ziel bestimmt die Auswahl der selbigen (Have 2004: 15), welches hier weit über reine Datengewinnung hinausreicht. Aufgrund der Einhaltung ethischer Prinzipien wird Vertrauen geschaffen. Dies gewährleistet die Ehrlichkeit der Aussagen und damit die Reliabilität der Daten. Diese Verlässlichkeit zeichnet die Qualität der gewonnenen Daten aus. Die Prioritätenverschiebung von einer engstirnig wissenschaftlich-technischen Ausrichtung auf methodische Güte hin zu Ethik in der Forschung erzielt höhere Verlässlichkeit, begründet die Brauchbarkeit der gewonnenen Daten und hebt somit umfassend die Forschungsqualität.

Partizipative Methodenpakete wie participatory rural appraisal (PRA), participatory learning and action (PLA) oder community action research (CAR) stellen die Vorteile von Visualisierungen in ihren gruppenbasierten Methoden heraus (Kumar 2002: 44;

\footnotetext{
13 Güte bezeichnet hierbei das Verständnis qualitativer und quantitativer Sozialforschung, wonach Methoden entsprechend ihres intrinsischen Potentials zur Erzielung möglichst genauer bzw. aussagekräftiger Daten bewertet werden. Als Bewertungsmaßstab dienen jedoch primär technische Eigenschaften einer Methode, während ethische Dimensionen unberücksichtigt bleiben.
} 
IFRC 2007a: 75; Slocum 2003: 30). Kartierungen, Zeichnungen und graphische Anordnungen heben Beziehungen vielfältiger Sachverhalte hervor. Zudem können sich weniger eloquente und nicht schreibsichere Teilnehmer einbringen. Da sie das Thema permanent vor Augen haben, arbeiten sie es zielstrebig, effizient, umfassend und mit Übersicht heraus. Weil die Visualisierung von den Teilnehmern übernommen wird, drückt das Medium ihre Ansichten, Prioritäten und Realitätswahrnehmungen aus. Dies steigert auch das kulturelle Verständnis des Forschers.

Triangulation ist ein weiteres Prinzip partizipativer Forschung, umfassende und verlässliche Ergebnisse zu erzielen. Das Triangulieren bezieht sich zum einen auf die Anwendung verschiedener Methoden zu gleichen Thematiken, zum anderen auf die Wiederholung von Methoden mit unterschiedlichen Fokusgruppen. Gerade die Kombination aus teilnehmender Beobachtung und partizipativer Methodenpakete birgt Potential, Unsicherheiten und Verzerrungen zu vermeiden.

Zur Bewertung der Forschungsqualität einer partizipativen Herangehensweise ist zu beachten, dass eine Wiederholbarkeit der Untersuchung nur bedingt möglich ist und die erzielten Ergebnisse nicht repräsentativ sind. Damit sind zwei Gütekriterien quantitativen wissenschaftlichen Arbeitens nicht erfüllt. Diesen Anspruch hat partizipative Sozialforschung aber niemals besessen bzw. kann und möchte bereits qualitative Sozialforschung ohnehin nicht leisten. Auch der Grad der Korrelation mit vorherigen Untersuchungen, ein typisches Gütekriterium quantitativer Forschung, stellt hier kein Qualitätsmerkmal dar. So will man in der Regel exakter als vorhergehende Untersuchungen einen Gegenstand erfassen; man möchte abweichende Ergebnisse erzielen. Zudem ist qualitative und insbesondere partizipative Forschung kontextgebunden, Ergebnisse sind situativ und verändern sich daher in Raum und Zeit (Mayring 2002: 140-142).

\subsection{Aufbau der Forschungsmethoden}

Ich habe über drei Forschungsaufenthalte verteilt zwischen Dezember 2009 und Februar 2013 insgesamt 14 Monate auf den Fidschi-Inseln verbracht. Über die Hälfte der Zeit habe ich in Dörfern gelebt, den Rest der Zeit verbrachte ich vornehmlich in der Hauptstadt Suva in engem Kontakt zur örtlichen Universität und zu Verwandten meiner Gastfamilien aus den Untersuchungsdörfern.

Zentral für diese Untersuchung sind Forschungserhebungen in drei Standorten. Zur Erfassung umfangreicher Erkenntnisse in die gesellschaftlichen Transformationsprozesse unterscheiden sich die drei Dörfer in ihrer Abgeschiedenheit vom urbanen, wirtschaftlichen und politischen Zentrum Fidschis, der Hauptstadt Suva. ${ }^{14}$ Der erste Standort ist Malawai auf der entlegenen Insel Gau Island, welche etwa einmal monatlich von der Hauptstadt Suva aus mit einem Transportschiff

\footnotetext{
${ }^{14}$ Diese Beschreibung ist eine Darstellungsform für die mit den lokalen Begebenheiten unvertraute Leserschaft. In der Wahrnehmung der Dorfbewohner ist die örtliche Beschaffenheit nicht adäquat mittels einer Lagebeziehung zur Hauptstadt Suva charakterisiert.
} 
angesteuert wird. Nabuna auf Koro Island ist der zweite Forschungsstandort. Die Insel liegt auf der mehrmals wöchentlich tätigen Fährverbindung zwischen der Hauptstadt Suva und Savusavu bzw. den beiden größten Inseln Fidschis, Viti Levu und Vanua Levu. Der dritte Standort ist Dravuni an der Ostküste der Hauptinsel Viti Levu, etwa eine Autostunde von Suva entfernt (siehe Karte 1).

Es wird zunächst unterstellt, je peripherer die Dörfer gelegen sind, desto geringer seien sie von medialen und nationalpolitischen Einflüssen gekennzeichnet; desto größer seien Transport- und Marktzugangsprobleme, sodass nur geringfügige monetäre Einkommen erzielt werden können; desto geringer seien also die Auswirkungen der gesellschaftlichen Transformation. Spannend ist demzufolge auch die Frage, ob sich in diesem Zusammenhang die soziale Kohärenz in den Dörfern unterscheide. Durch die zunächst postulierte Unterschiedlichkeit der Untersuchungsdörfer wird der Differenziertheit der Grundthematik sozialer Sicherung bei gesellschaftlicher Transformation innerhalb Fidschis in sozialen politischen, wirtschaftlichen und räumlichen Dimensionen Rechnung getragen.

Tabelle 1: Übersicht wichtiger Forschungsstandorte

\begin{tabular}{|l|l|l|}
\hline Ortsname & Lagecharakteristika & Aufenthalte \\
\hline Malawai, Gau Island & Küstendorf auf kleiner, entlegener & 20.12 .2009 bis 25.01 .2010 \\
& Außeninsel & 07.01 .2012 bis 15.01 .2012 \\
& & 10.01 .2013 bis 17.01 .2013 \\
\hline Nabuna, Koro Island & Küstendorf auf kleiner, ange- & 02.12 .2011 bis 05.01 .2012 \\
& schlossener Außeninsel & 30.08 .2012 bis 02.09 .2012 \\
& & 14.09 .2012 bis 21.09 .2012 \\
& & 25.01 .2013 bis 03.02 .2013 \\
\hline Dravuni, Viti Levu & Küstendorf auf Hauptinsel, mit & 28.11 .2012 bis 03.01 .2013 \\
& Hauptstadt vernetzt & 08.02 .2013 bis 15.02 .2013 \\
\hline Tabuya, Kadavu & Küstendorf, peripher auf großer & 25.01 .2012 bis 01.02 .2012 \\
& Außeninsel & 28.10 .2012 bis 05.11 .2012 \\
\hline Nukui, Viti Levu & Küstendorf auf Hauptinsel, an & 05.02 .2010 bis 19.02 .2010 \\
& Hauptstadt angeschlossen & 10.10 .2012 bis 15.10 .2012 \\
\hline Natavea, Viti Levu & Binnendorf auf Hauptinsel, an & 12.03 .2010 bis 17.03 .2010 \\
& Hauptstadt angebunden & \\
\hline
\end{tabular}

In den Hauptuntersuchungsdörfern startete die jeweilige Forschung mit einem fünfwöchigen Aufenthalt. In dieser Zeit lebte ich in Gastfamilien und betrieb in erster Linie ethnographische, teilnehmende Beobachtung. Es gab häufige Gelegenheiten, Nachbardörfer aufzusuchen, sodass gewonnene Eindrücke dort abgeglichen werden konnten, um möglichst fundierte Aussagen über das Leben in fidschianischen Küstendörfern insgesamt treffen zu können. Zu diesem Zwecke wurden auch weitere Dörfer aufgesucht und die Dörfer heterogen zusammengestellt (siehe Tabelle $1 \&$ Karte 1). 
Der Aufbau der Forschung in den drei Hauptdörfern ist vergleichbar. Aus ethischen und methodischen Gründen bietet sich solch eine Regelgeleitetheit (Mayring 2002: 145f.) an, bestimmte Reihenfolgen einzuhalten. Dies erhöht auch die Vergleichbarkeit der Dörfer. Auch der Zeitraum des jeweils ersten Forschungsaufenthalts wurde bewusst zur Weihnachtszeit gewählt um Verzerrungen zu reduzieren. Zuallererst stellte ein Fürsprecher mich und meine zuvor geschulten Forschungsassistenten der Dorfgemeinschaft in einem sevusevu (Willkommenszeremonie) vor. In der ersten Woche wurden Informationen ausschließlich durch teilnehmende Beobachtungen inklusive freundlicher, unstrukturierter Konversationen erzielt. Im Folgenden hatten Einladungen, an weiteren kulturellen Bräuchen wie Weihnachten, dem Neujahrsfest, Gottesdiensten, Hochzeiten und Beerdigungen teilzunehmen, stets Vorrang gegenüber sonstiger Methoden, da die kulturellen Einblicke in die soziale Gemeinschaft ein enormes Verständnis von sozialen Werten, Aktiva, Verwundbarkeiten und Problemdimensionen verschaffen. Zur Generierung von Alltagserfahrungen genossen Feldarbeit, Fischfang und Hausarbeiten ebenfalls hohe Priorität. Der Respekt für die kulturellen Werte wurde zudem sehr positiv aufgenommen, was die Eingliederung ins örtliche Leben erleichterte. An vielen kulturellen Ereignissen nahmen außerdem stets nahezu alle anderen Dorfbewohner teil, sodass die Bildung von Fokusgruppen zu diesen Zeiten schwierig zu gestalten gewesen wäre.

Ab der zweiten bis zur vierten Woche wurden zusätzlich verschiedene partizipative Methoden zumeist als Gruppendiskussionen mit Visualisierungen angewandt. Diese entlehnten sich zumeist dem PRA. Mehrere davon wurden in allen drei Dörfern angewandt, manche jedoch nur in ein oder zwei Dörfern. Da die jeweilige Methode einen unterschiedlichen inhaltlichen Schwerpunkt bearbeitet, liegt die flexible Handhabung in den unterschiedlichen Interessen, Herausforderungen und Spezifika des singulären Dorfes und seiner Einwohner begründet. Die einzelnen Methoden werden im nächsten Unterkapitel genauer vorgestellt.

In Nabuna und Dravuni wurde ab der vierten Woche zudem ein knapper, standardisierter Fragebogen zur Bedeutung sozialer Beziehungen für nachhaltige, menschenwürdige Entwicklung (siehe Anhang) mit 40 bzw. 20 Einzelpersonen bearbeitet. In den ca. 130 bzw. 70 Einwohner zählenden Dörfern wurde so jeder haushalt mit mindestens einer Person abgedeckt. Als Basis zur Erstellung des Fragebogens dienten die partizipativ erhobenen Erkenntnisse aus Malawai, ein working paper der Weltbank zur Messung von Sozialkapital (Grootaert et al. 2004) und Fragebögen zur Wahrnehmung vom Klimawandel und Naturgefahren im Pazifik $^{15}$. Das erste Interview wurde beide Male mit dem turaga ni koro (Ortsvorsteher) geführt, um sich dessen Segen für weitere Befragungen abzuholen. Die geführten Interviews hatten jedoch keinen standardisierten Charakter, vielmehr

\footnotetext{
${ }^{15}$ Diese wurden von Katharina Beyerl im Rahmen ihrer Promotion zur Wahrnehmung von Umweltveränderungen in pazifischen Inselstaaten und von Eberhard Weber zur Untersuchung von Überflutungen fidschianischer Küstenstädte erstellt (unveröffentlicht).
} 
wurden die Fragekategorien als Leitfäden für offene Interviews genutzt, sodass die Gespräche, je nach persönlicher Motivation und Zeit des Gegenübers, zwischen 40 Minuten und zwei Stunden dauerten. Diese Handhabung sollte den Respekt und die Empathie für den individuellen Gesprächsteilnehmer ausdrücken und damit die in den vorherigen Wochen geschaffene Vertrauensbasis aufrechthalten (Meier Kruker \& Rauh 2005: 62f.). Zudem liefert dieses Vorgehen entscheidende Hintergrundinformationen, da diese Studie nicht nur auf Beschreibungen aus ist, wie aktuelle Begebenheiten wahrgenommen werden, sondern vor allem erklären will, warum Phänomene entsprechend beurteilt werden.

Sämtliche Methoden wurden gemeinsam mit den Forschungsassistenten angewandt und regelmäßig Kurzreflektionen gehalten. In der fünften Woche fand eine Gesamtreflektion statt. Dabei herauskristallisierte Wissenslücken wurden in jener letzten Woche durch informelle Gespräche freundlichen Charakters über dieselbe Thematik mit Einzelpersonen verkleinert. Abschließend wurden die gesammelten Erkenntnisse der Dorfgemeinschaft im Rahmen eines Abschiedsfestes präsentiert und diskutiert.

Malawai und Nabuna wurden regelmäßig weitere Besuche abgestattet. Sämtliche der den fünfwöchigen Forschungsphasen nachfolgenden Aufenthalte fanden ohne Forschungsassistenten statt. PRA-Methoden wurden keine mehr angewandt und die persönliche Motivation überlagerte die wissenschaftliche. Diese Bestätigung des freundschaftlichen Interesses und der gewollte Wegfall künstlicher Forschungssituationen mittels PRA-Techniken und Forschungsassistenten festigte ungemein das Vertrauen in mich. Die Ehrlichkeit, dass ich zuvor keine unrealistischen Versprechungen über den Nutzen der Studie für die Dorfbewohner gegeben hatte, welche ich nicht einlösen konnte, und dass ich mein Wort hielt, weiterhin den Kontakt halten zu wollen, brachte mir viel Anerkennung. Bei den Wiederdaseinsfeiern und dem gemeinsamen Sinnieren über vergangene Aufenthalte, erhielt ich nebenbei Informationen und Perspektiven, die unabdingbar für die Ergebnisse der hiesigen Studie sind. Auf dieser persönlichen Erfahrung fußen die Erkenntnisse, dass zum einen die Identifikation des Forschers mit seinem Forschungsumfeld, und zum anderen die Unterordnung von Erhebungen unter ethische Gebote, enorm die Forschungsqualität in interkulturellen Kontexten stärken.

Zahlen zur ortsansässigen Bevölkerung sind schwankend, viele Verwandte wohnen in der Hauptstadt Suva, in Tourismusgebieten oder auch in Übersee. Speziell die Verwandten in Suva werden häufig besucht und es entstehen zirkuläre Wanderungen zwischen den Orten (siehe Kap. 6.5). Dadurch, dass ein Zeitraum von 38 Monaten in Malawai und 14 Monaten in Nabuna persönlich abgedeckt ist, sich abseits der Forschungszeiträume Freundschaften zu manchen in Suva lebenden Verwandten entstanden und Kontakte bis heute halten, können Dynamiken und Entwicklungen in beiden Dörfern wahrgenommen, nachvollzogen und in diese Studie eingearbeitet werden. Als Korrektiv dienen hier zusätzlich die zweifachen Aufenthalte in Nukui und Tabuya, mit einzelnen dauerhaft weiterbestehenden Freundschaften. Aufgrund der zeitversetzten Hinzunahme der Untersu- 
chungsdörfer war ein gleichwertiger Aufbau langfristiger Beziehungen zum neuesten Untersuchungsdorf Dravuni nicht möglich. Wegen der fortgeschrittenen Erfahrungen im ethischen Verhalten sind die dort geknüpften Freundschaften jedoch nicht weniger intensiv. Während die Abschlusspräsentationen am Ende der fünfwöchigen Aufenthalte speziell auf das jeweilige Dorf ausgerichtet waren, wurden am Ende der Gesamtuntersuchung nochmals alle drei Dörfer aufgesucht und die gewonnenen, vorläufigen Ergebnisse insgesamt vorgestellt und mehrstündig diskutiert.

\subsection{Angewandte partizipative (PRA-) Methoden}

Den Kern von PRA bildet die Gruppendiskussion über ein bestimmtes Thema bei gleichzeitiger Visualisierung. Anhand der Themenstellung und ausgewählten Methode ist die Diskussionsstruktur in Teilen vorbestimmt. Durch die Auswahl der Teilnehmer in Fokusgruppen wird ebenfalls Einfluss genommen. Die Inhalte der Diskussion werden jedoch ausschließlich von den Teilnehmern gefüllt. Die Fokusgruppen wurden im Sinne möglicher Verwundbarkeitsgruppen nach Alter oder Geschlecht unterschieden, zum Teil konnte zusätzlich nach Konfessions- und mataqali (Klan-)Zugehörigkeit differenziert werden. Sofern nicht anders erwähnt, wurden sämtliche Methoden mit zwei bis sieben Teilnehmern durchgeführt, wobei es mitunter zu Fluktuationen innerhalb der Fokusgruppe kam, was der Idee von PRA jedoch nicht entgegensteht. Zur Visualisierung wurden je nach Bedarf Papierbögen, Karteikarten unterschiedlicher Größe und bunte Stifte ausgeteilt. Die Digitalisierung der Visualisierungen in dieser Arbeit sollen die Werke der Partizipanten möglichst originalgetreu wiederspiegeln um Manipulationen zu vermeiden. Trotz mitunter guter Englischkenntnisse vor allem der jüngeren Bevölkerung wurden die Diskussionen nahezu ausschließlich auf Fidschianisch gehalten, um eine angenehme Atmosphäre zu kreieren. Da ich mir im Laufe der Zeit lediglich Grundkenntnisse aneignen konnte, vertraut diese Studie hier sehr auf die Fähigkeiten der Forschungsassistenten. Die folgenden Methoden wurden in Anlehnung an Somesh Kumars diesbezügliches Handbuch ausgewählt und praktiziert. Er unterscheidet die Methoden hinsichtlich einer Analyse des Raumes, der Zeit und von Relationen (Kumar 2002).

In jedem der drei Untersuchungsdörfer startete ich mit Problem-Scorings. Diese relationale Methode listet Herausforderungen im Alltag der jeweiligen Fokusgruppe und soll deren Bedeutung auf einer Skala messen. Dies verschaffte den Überblick über Problemdimensionen. Weiterhin sollte mit Pfeilen ein Trend angegeben werden, inwiefern sich das Problem in den letzten fünf bis zehn Jahren veränderte, sodass sich der Erkenntnisgewinn um zeitliche Dimensionen erweiterte. In Malawai initiierte ich zusätzlich rein ökologische Problem-Rankings, da ökologische Dimensionen zuvor weitestgehend außen vor blieben. Dies geschah noch im Rahmen meiner Magisterarbeit, weil ich hier ein Thema, „Verwundbarkeit gegenüber Naturgefahren", vorkonzipiert hatte. Wie auch bei jeder nachfol- 
gend beschriebenen Methode steht nicht nur das graphische Endprodukt, sondern genauso die Diskussion darüber im Fokus, warum Phänomene benannt und wieso sie entsprechend bewertet werden. Dies schafft parallel tiefere Einblicke in die kulturellen Wertvorstellungen.

Venn-Diagramme sozialer Institutionen als weitere relationale Methode wurden ebenfalls in allen Dörfern angewandt. Sie bilden die Bedeutung formeller und informeller Gliederungseinheiten des Sozialgefüges und jeweilige Zugangsmöglichkeiten ab. Für den Erhalt tiefergehender Einblicke in das soziale Leben wurden zudem Auflistungen bedeutender Einzelpersonen für die Dorfgemeinschaften angestoßen. Aus ethischen Gründen wurde auf die Erstellung einer Rangfolge der Personen verzichtet.

Zur Erfassung des Alltags in zeitlichen Dimensionen wurden Ehepartner gebeten, ihre Aktivitäten der letzten Woche aufzulisten. Dies sollte Einblicke in Arbeitsteilungen und Interaktionen von Eheleuten untereinander, aber auch mit der weiteren Gemeinschaft gewähren und Prioritätensetzungen im Zeitmanagement erklären. Das Leben gestaltet sich in den Dörfern zu unterschiedlich, als das die Erstellung typischer Tages- oder Wochenabläufe in Gruppendiskussionen als alternative Methoden sinnvoll erschien. Zur Erfassung historischer Dimensionen und als bedeutend wahrgenommener Ereignisse wurden in Malawai und Nabuna Zeitstrahle der Dorfgeschichte mit Dorfältesten entwickelt. In Dravuni sammelte ich bereits in alltäglichen Diskussionen umfangreiche Informationen zur dörflichen Historie, sodass zusätzliche PRA-Methoden dahingehend nicht forciert wurden.

Zur Erfassung räumlicher Dimensionen wurden in allen drei Orten Dorfkarten angefertigt. Diese wurden jedoch nicht mehrfach pro Dorf mit unterschiedlichen Fokusgruppen erstellt, sondern einmalig von mir und meinen Assistenten unter Rücksprache mit der Dorfjugend. Anschließend wurde die Dorfkarte weiteren Dorfbewohnern gezeigt und um deren Verbesserungsvorschläge ergänzt. Da weitreichende Familiennetzwerke eine große Bedeutung für das örtliche Leben haben, wurden diese mit in die Familien eingeheirateten Mitgliedern (zumeist Frauen) auf Landkarten nachgehalten. Diese Familiennetzwerk-Kartierungen wurden in Nabuna und Dravuni durchgeführt. Aufgrund mannigfaltiger Beziehungen Nabunas zu sämtlichen weiteren Dörfern Koro Islands, wurde eine Koro Island-Karte zur Klärung von Beziehungsformen und Intensitäten zwischen den Dörfern mit einem Dorfältesten erstellt.

In Malawai standen Naturgefahren zentraler im Fokus der Betrachtung als in den anderen Dörfern, wo ein offenerer Zugang gewählt wurde. Aufgrund der zu diesem Zeitpunkt mangelnden persönlichen Erfahrungen mit tropischen Zyklonen, ihren Auswirkungen und ihrer Bedeutung für das örtliche Leben, lies ich Impact-Diagramme tropischer Zyklone erstellen und zudem eine Risiko-Karte vom Dorf anfertigen. Letztere konnte triangulierend potenzielle Verzerrungen intersubjektiver Wahrnehmung hinsichtlich der selbsterstellten Dorfkarte negieren. 
In Nabuna und Dravuni wurden weiterhin zwei Methoden zur Erfassung kultureller Werte angewandt. In Werte-Priorisierungen sollte die Dorfjugend in Kleingruppen Werte niederschreiben und priorisieren. Anschließend stellten die Kleingruppen ihre Ergebnisse im Plenum vor und diskutierten diese. Dies gewährt spielerisch Einblicke in Werte und Formen von Konfliktlösungen. Kerekere beschreibt einen weitverbreiteten Brauch, Bitten nicht abschlagen zu dürfen (siehe Kap. 8.2). In entsprechenden kerekere-Diagrammen wurden Beispiele für Bitten gelistet und die Vorzüge und Nachteile dieses Brauches diskutiert.

\subsection{Kritisches Fazit zur Methodik}

Die Erkenntnis der Bedeutung ethischen Verhaltens auf die Qualität einer Datenerhebung ist erst während des Forschungsprozesses in vollem Umfang realisiert worden. So stand bei meinem ersten Gehversuch zur Magisterarbeit noch die reine Datenerhebung im Vordergrund. Dies führte zu ethischen Dilemmata und konterkarierte den wissenschaftlichen Erkenntnisgewinn. Mangelndes Gespür für günstige Zeitansetzungen von Gruppendiskussionen sorgte beispielsweise für dürftige inhaltliche Tiefe bei zwei Methoden mit Frauengruppen. Auch Vorstellungen über sensible Themen sprechen zu können, ohne sich hinreichend Mühe zu geben, deren erhoffte Bedeutung für die Studie ausführlich im Vorfeld zu begründen, resultierten zu Recht in Zurückhaltung. Glücklicherweise konnten die vier Forschungsassistenten korrektiv eingreifen, um mögliche negative Deutungen meines Verhaltens zu entkräften und den Sinn der Studie hervorzuheben. Gespräche mit den Assistenten beugten auch möglichen Fehlinterpretationen meinerseits hierbei beispielsweise über die Rolle von Frauen oder zwischengeschlechtlicher Kommunikation vor. Zudem halfen sie im Haushalt und der Landwirtschaft viel gekonnter und selbstverständlicher mit, als dies mir im unvertrauten Umfeld möglich gewesen wäre, womit sie das Wohlwollen und Vertrauen in die gesamte Forschungsgruppe förderten. Gerade im ersten Untersuchungsdorf war das eigene Verhalten noch sehr von kulturell unpassendem Verhalten geprägt, was Dank der grundsätzlichen Wohlgesinnung sowie der Arbeit meiner Fürsprecher und Assistenten als belustigend und nicht beleidigend aufgefasst wurde. Da ich nicht der erste Europäer war, der zu Forschungszwecken nach Malawai vermittelt wurde, existierte seitens der Dorfbewohner bereits ein ausgeprägtes Verständnis für interkulturelle Schwierigkeiten. Dies zeigt, wie abhängig die Qualität partizipativer Forschung in interkulturellen Kontexten gerade zu Anfang von der Wahl der Forschungsassistenten und Fürsprecher ist.

Auch die anfänglich gewonnenen Daten finden Einklang in diese Studie, werden jedoch nicht vorbehaltlos eingebaut. Generell finden die Interpretationen aller partizipativ erhobenen Daten vor dem Hintergrund der gesamten gemachten Erfahrungen statt. Aus Effizienzgründen wird hier nicht jede Erfahrung im vollen Umfang niedergeschrieben, sondern die Erkenntnisse gebündelt und geordnet komprimiert wiedergegeben. Aus moralischer Verschwiegenheit können manche 
Erklärungen zudem nicht in ihrer Tiefe ausgeschöpft werden; dabei soll jedoch die Nachvollziehbarkeit gewährleistet bleiben.

\subsubsection{Limitationen der Güte der Forschung}

Eine völlige Anpassung des Forschers an seine Umgebung ist nicht möglich (siehe Abb. 1), weshalb er sich den Verzerrungen, die er selbst unweigerlich hervorruft, bewusst sein muss, damit die Ergebnisse dies berücksichtigen. Der Einfluss der eigenen Person auf die Aussagen der Teilnehmer darf trotz der zurückhaltenden, lediglich vermittelnden Stellung nicht unterschätzt werden. Die Diskussionsteilnehmer sind stets mit einer gewissen Erwartungshaltung an meine Person in die Diskussion gegangen. Mir wurde mitunter hoher Respekt gezollt, der sich in Zurückhaltung äußerte. Zum Teil hielt eine Grundskepsis die gesamte Zeit über vor und die Menschen fragten nach, ob ihre Aussagen meine Erwartungen erfüllen. Dies steht den Ideen von PRA diametral entgegen.
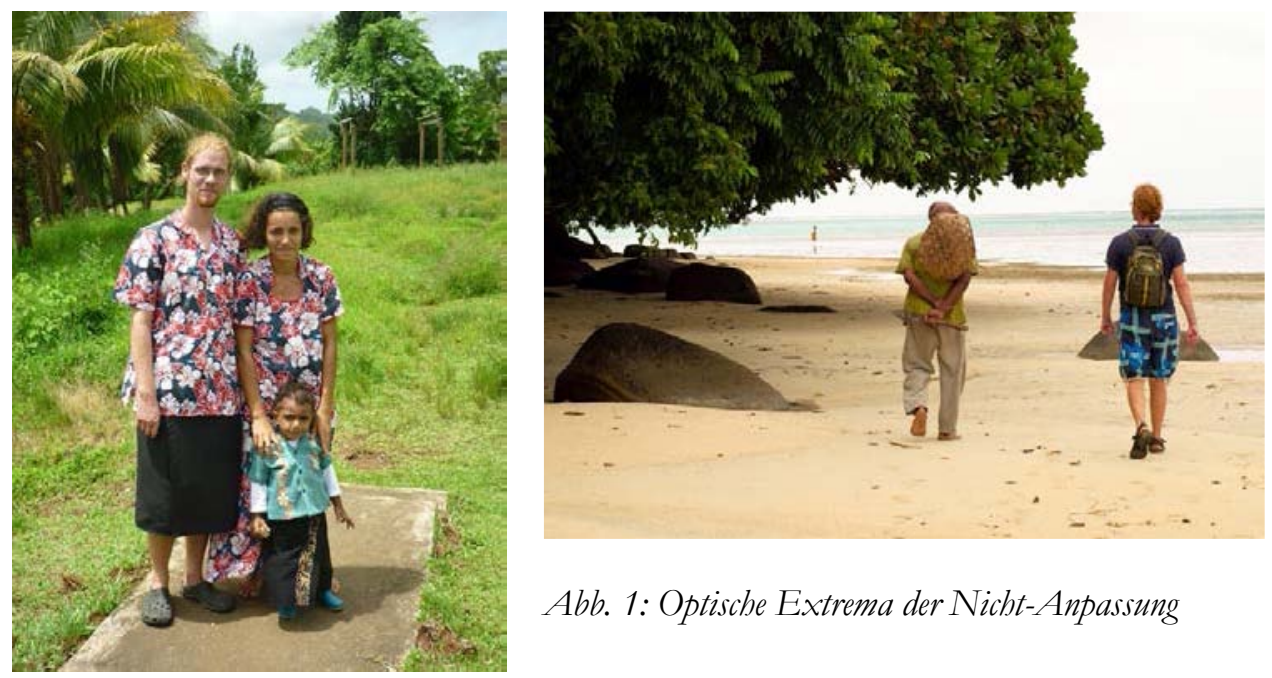

Abb. 1: Optische Extrema der Nicht-Anpassung

Eigene Meinungen seitens der Diskussionsteilnehmer zu bestimmten Themen bilden sich zum Teil erst im Laufe der Diskussion und sind daher abhängig von den Gesprächspartnern. Der Wunsch nach homogener Zusammenstellung in Gruppendiskussionen kann hier zu extremen Positionen führen, die sich im natürlicherweise heterogeneren Umfeld nicht gebildet hätten. PRA hat somit den Hang, dass Randpositionen überrepräsentiert sind und soziale Verwundbarkeit übertrieben wird, sodass der Forscher seine Ergebnisse diesbezüglich reflektieren muss. Man schafft zudem künstliche Situationen (siehe Kap. 7.1.1) Dennoch sollte die Homogenität in den Gruppen gewahrt bleiben, um diskussionsfähige Teilnehmer zu gewinnen, wobei man sich jedoch möglicher Schwachstellen des Ansatzes bewusst sein sollte. 
Es wurde nicht verschwiegen, dass die Ergebnisse der Studie auch im Rahmen einer Dissertation für ein größeres Publikum zugänglich gemacht werden sollen. Dies kann Erwartungen an die Studie wecken, die ich jedoch klein bzw. realistisch halten wollte. Unterschiedlichste Motivationen der Diskutanten können Einfluss auf die Erkenntnisdarstellung nehmen. So könnte es einen Wunsch geben, die sich bietende Plattform zur positiven Selbstdarstellung oder zur Frustbewältigung zu nutzen. Ebenso kann man, in der Hoffnung auf Besserung durch Auswärtige, Unstimmigkeiten skandalisieren, Herausforderungen überbetonen und eigene Bewältigungspotentiale kleinreden, oder aber genau umgekehrt der Gruppe Mut und Hoffnung in die eigenen Fähigkeiten zur Überwindung von Herausforderungen zusprechen wollen. Genauso gut kann es sein, dass Problemfelder unbehandelt bleiben, weil die Gruppenmitglieder dies untereinander nicht diskutieren wollen, dem Forscher bzw. der Öffentlichkeit private und intime persönliche Details verbergen wollen, oder einen Bezug zur Studie verkennen. Eine Klarheit über die Motivationen der Partizipanten bleibt dem Forscher in der Regel verwehrt. Eine generelle Unterstellung wiederum, der Forschungsprozess sei - unbewusst oder bewusst - beeinflusst bzw. manipuliert worden und die Resultate würden nicht die wahrgenommenen Realitäten wiederspiegeln, würde die Vertrauensbasis negieren. Dies ist ethisch nicht tragbar. Damit bleiben die explizit partizipativen Methoden mit Unsicherheiten versehen, welche jedoch mittels Triangulation gering gehalten werden.

\subsubsection{Machtungleichbeiten und mangelnder Partizipationsgrad bei Gruppendiskussionen und Visualisierungen}

Gerade die gängigen Schulen partizipativer Forschung wie PRA, PLA und CAR (Disaster Mitigation for Sustainable Livelihoods Programme 2008; IFRC 2007a; IFRC 2007b; Kumar 2002; McFadzien et al. 2009; Slocum 2003) werden meineserachtens ihren eigenen Ansprüchen an Machtegalität und dem Grad an Partizipation nicht gerecht. Beim praktischen Anwendungsbezug genießt die reine Datenerhebung hier noch zu hohen Stellenwert. Die ethischen Dilemmata, die dies hervorruft, bleiben unberücksichtigt. Dass die Dominanz des Forschers bei der Anwendung partizipativer Methoden durch dessen Zurückhaltung in inhaltlichen Diskussionen nivelliert wird, ist ein Trugschluss. Wenn die Datenerhebung im Vordergrund steht, muss der Forscher seine Intention unter Umständen verbergen, um differenzierte Antworten zu erhalten. Er hält bewusst Informationen beispielsweise aus vorherigen Methodenanwendungen mit anderen Fokusgruppen - zurück, um die Ergebnisse nicht zu beeinflussen. Sollte eine Methode mit einer weiteren Gruppe wiederholt werden, muss der Forscher verweigern, bereits erworbenes Wissen preiszugeben, um die Offenheit sicherzustellen. Dadurch bleiben ungleiche Machtverhältnisse bestehen.

Die Passivität des Mediators kann als Störfaktor wahrgenommen werden, Unsicherheiten hervorrufen, da eine Bestätigung der Richtigkeit und Verständlichkeit 
eigener Aussagen ausbleibt. Dies kann zur Zurückhaltung seitens der Diskutanten führen und damit dem Ziel der Methode entgegenstehen. Als Beobachter steht der Mediator außerhalb der eigentlichen Diskussion bzw. über dem Geschehen, symbolisch untermauert durch Notizblock und Stift. Partizipation wird in dieser Studie nicht nur als ein soziales Miteinander der Fokusgruppenmitglieder verstanden, sondern bezieht auch die Teilhabe des (Haupt-)Forschers ein. Übt er sich in Zurückhaltung, partizipiert er nicht im möglichen Umfang an der Methode. PRA wird daher den eigenen Ansprüchen an Partizipation nicht gerecht.

Zur Vermeidung von Verzerrungen und Unschärfen muss humanwissenschaftliche Forschung im natürlichen, alltäglichen Umfeld der Untersuchten durchgeführt werden (Mayring 2002: 22). Im Sinne der Hermeneutik gibt es keine absolute Objektivität in der Forschung, da die Realität unterschiedlich wahrgenommen wird. Laborsituationen würden diese Verzerrungen verstärken und die Schaffung künstlicher Situationen vor Ort tut dies ebenfalls. Gerade bei angeregten Gruppendiskussionen mit Visualisierungen sind sich die Partizipanten der Künstlichkeit der Situation bewusst; sie wissen, dass sie sich in einer Forschungssituation befinden, reflektieren dies und es beeinflusst ihre Handlungen. Einen weiteren Widerspruch zum Ziel, unverfälscht und egalitär Ansichten und Bedürfnisse der Teilnehmer erfassen zu wollen, stellt der Fakt dar, dass der Initiator der Forschung sowohl die Grundthematik diktiert, als auch die Form der Visualisierung beispielsweise als Karte, Tabelle oder Wirkungsdiagramm vorbestimmt und einleitet. Eine Künstlichkeit der Diskussionssituation bleibt daher bestehen und Machtungleichheiten werden so allenfalls gemindert, aber nicht nivelliert.

\subsubsection{Zur Rechtfertigung von Machtungleichgewichten in der Forschung}

Ich habe im Rahmen dieser Studie viel mit Gruppendiskussionen und Visualisierungen gearbeitet, ehe ich mir der Tragweite dieser Machthierarchien hinreichend bewusst wurde. Eine Konsequenz wäre es daher auf Gruppendiskussionen und Visualisierung zu verzichten und mittels teilnehmender Beobachtung gänzlich abzuwarten, bis man wichtigen Diskussionen beiwohnt, welche im natürlichen Miteinander der Dorfbewohner entstehen. Aus folgenden Gründen wurde dennoch nicht darauf verzichtet:

Nach meinem Selbstverständnis und dem Verständnis der Dorfbewohner, bin ich als Forscher angereist, habe diese Absichten erläutert und die Einwilligung der Menschen vor Ort bekommen, die Forschung zu betreiben. Damit einhergehend sind mir Rechte eingeräumt worden, Fragen stellen zu dürfen und Gruppendiskussionen zu erbitten. Gruppendiskussionen und Visualisierungen wurden nie in der ersten Woche im Dorf abgehalten, sondern erst, nachdem gewisse persönliche Beziehungen aufgebaut waren und sich das gegenseitige Vertrauen manifestierte. Der Sinn meines Aufenthaltes war zu diesem Zeitpunkt den meisten Beteiligten soweit klar, dass sie gerne an der Forschung teilnahmen. Den Beitrag der jeweiligen Methode zum Forschungsziel hatte ich nach bestem Wissen versucht deutlich 
zu machen. Ich habe stets herausgestellt, dass es der Studie um ihren Lebensalltag geht und sie auf diesem Gebiet die Experten sind, ich selbst inhaltlich wenig beisteuern könnte und daher auf ihre Partizipation angewiesen sei. Je mehr ich mir der trotzdem bestandenen Künstlichkeit und Machtungleichheit bewusst wurde, desto intensiver bin ich auf die methodologischen Intentionen der Zurückhaltung und Offenheit eingegangen. Diese umfassende Herangehensweise schaffte das nötige Verständnis für die Methoden und die Passivität meiner Rolle. Dies schuf Akzeptanz, sodass die Teilnehmenden die Machtungleichheiten tolerierten und legitimierten, sodass das Vertrauen nicht erodierte, sondern sich dank meiner Ehrlichkeit im Gegenteil steigerte. Gegen Ende der Forschungsaufenthalte im jeweiligen Dorf war das Vertrauen in mich und die Studie jeweils so groß, dass ich in zwei Dörfern auch knappe standardisierte Einzelinterviews ausprobierte. Bei entsprechender Anleitung, die auf bestehende Machtungleichheiten in der Interviewsituation einging, stießen sie durchweg auf Verständnis und Akzeptanz, weshalb sie vermutlich keine forschungsethischen Verwerfungen hervorriefen.

Mit dem Ergebnis, dass Machtungleichheiten nicht zwangsläufig ethisch verwerflich sind, geht eine allgemeine machttheoretische Erkenntnis einher. Menschen können Macht von anderen Menschen legitim erhalten, sodass Machtunterschiede einen gesellschaftlich erwünschten Zustand beschreiben können. Macht als solche ist normativ nicht negativ zu bewerten, lediglich ein Missbrauch dieser Macht, beispielsweise zur persönlichen Bereicherung auf Kosten anderer.

Bezogen auf die vorliegende Dissertationsschrift habe ich letztlich trotz intensiver Rücksprachen mit den Menschen vor Ort den alleinigen Gesamtüberblick. Obwohl die Dorfbewohner ihre Erkenntnisse visualisierten obliegt mir die Darstellungshoheit und eine erhebliche Deutungs- bzw. Interpretationsmacht. Solch eine Schlüsselposition als Wissenschaftler mit erhöhter Macht muss jedoch nicht zwingend als Problem gewertet werden. Es kann im Gegenteil einen Wunschzustand seitens der mitforschenden Dorfbewohner darstellen, sofern man mich für fähig hält, das mir anvertraute Wissen und die mir verliehene Macht nach ihrem Willen bzw. unserem gemeinsamen Willen zu nutzen.

Ich teile entsprechende Forderungen, partizipative Forschung müsse auf eine Kommunikation auf Augenhöhe abzielen. Dies impliziert, die ungleichen Machtverhältnisse zu reduzieren, die die Beziehungen zwischen Experten und Befragten nicht-partizipativer Studien kennzeichnen, nicht jedoch ihre Nivellierung.

Auch eine Neutralität stellt kein Qualitätsmerkmal des Praktizierenden dar. Gegenseitiges Vertrauen setzt Empathie und Emotionalität voraus. Entwicklungspraktische, emanzipatorische und damit politische Zielsetzungen, beispielsweise hinsichtlich der Stärkung von empowerment, Eigentümerschaft, Demokratisierung und Bürgerschaft - in dieser Studie vor allem als öffentlicher Vernunftgebrauch aufgefasst (siehe Kap. 4.4) - sind legitime Bestandteile partizipativer Forschung und setzen eine begrenzte Einmischung voraus (Hickey \& Mohan 2004: 6-9; Waddington \& Mohan 2004: 220). 


\subsection{4 Überwindungsversuche des mangelnden Grades an Partizipation}

Zur Lösung des forschungsmethodischen und ethischen Dilemmas mangelnder Partizipation bei visualisierten Gruppendiskussionen muss der Initiator den Sinn seiner passiven Rolle und seiner Zurückhaltung bei inhaltlichen Diskussionen erklären, um Verständnis seitens der Diskutanten zu kreieren. Die hiesige Studie unterlag diesbezüglich einer Qualitätssteigerung im Verlauf des Forschungsprozesses.

Die alltägliche Teilhabe am öffentlichen Leben ist partizipativer und ethisch hochwertiger als PRA-Techniken. Diese Studie begreift Menschen als soziale Wesen. Ihr Wunsch, am Alltag ihres Umfeldes teilhaben zu dürfen, ist normativ verständlich und akzeptiert. Hierbei kann, soll und darf man seine Persönlichkeit einbringen. Dies schafft Freiheiten für den Forscher und ehrliche Einblicke in dessen Persönlichkeit. Können dessen Motive als ehrbar empfunden werden, so genießt er Vertrauen und Anerkennung. Aufgrund unüberwindbarer optischer und kultureller Unterschiede (siehe Abb. 1) und der zeitlichen Begrenzung seines Aufenthalts, bleibt der Forscher zu einem gewissen Grade Außenseiter. Auch wenn er sich mit dem Umfeld identifiziert, bleibt er in vielerlei Hinsicht bezuglos. Diese semi-neutrale Außenperspektive kann seitens seines Umfeldes begrüßt und geschätzt werden. Er sollte sich daher in der alltäglichen Kommunikation nicht zurückhalten - dabei jedoch natürlich kulturelle Gebote angemessener Kommunikation achten -, sondern seine eigenen Erfahrungen und daraus gewonnenen, kulturell geprägten Wertvorstellungen einbringen. ${ }^{16}$ Eine solche Aufrichtigkeit und Emotionalität schafft Vertrauen. Aufgrund von Sympathien mit dem Forscher und der Forschung hilft man ihm und gewährt ihm Einblicke in die Kultur und die damit verbundenen Wertvorstellungen. Solche explizite Gesten zur gegenseitigen Bewusstmachung der eigenen Kultur verschaffen beiden Seiten tiefe Einblicke. Diese Spiegelfunktion des Forschers hilft den Dorfbewohnern, Werte und Praktiken der eigenen Kultur kritisch zu reflektieren und implizites, kulturelles Wissen aktiv nutzbar zu machen, sodass entwicklungspraktisch empowerment gesteigert wird. Umgekehrt bekommt der Forscher ein ungemeines Verständnis örtlicher Begebenheiten, was seiner Integration und seiner Forschung gleichermaßen hilft.

Im Hinblick auf den Eurozentrismus birgt die persönliche Vernetzung jedoch auch Gefahren. So kann die weitreichende Partizipation dazu genutzt werden, eigene, eurozentristische Ideale von Entwicklung umzusetzen. Ich hoffe, Gespräche, in denen ich meine Wertvorstellungen implizit oder explizit erläutert habe, wurden in Sinne von Pluralität als Bereicherung empfunden. Auch aufgrund der Freiwilligkeit, auf dessen Basis unser Zusammenleben stattfand, denke ich, keine Zwänge und Fehlentwicklungen erzeugt zu haben.

\footnotetext{
${ }^{16}$ Ich wurde beispielsweise häufiger zu meiner Meinung zum politischen Systems Fidschis befragt. Hierzu verwies ich auf geschichtliche Erfahrungen meiner Kultur, vor dessen Hintergrund sich meine grundlegende Skepsis gegenüber Diktaturen erklärt.
} 
Die Partizipation am Alltag, sowie insbesondere an kulturellen Ereignissen dient hervorragend einer Forschung über Lebensqualität und Wohlbefinden in interkulturellen Kontexten. Die Stärken partizipativer Methodenpakete liegen hingegen in ihrer Effizienz, schnell und materialarm vor Ort Erkenntnisse zu erzielen. Mit ihrer Hilfe kann man insbesondere Überblicke und Dimensionen erfassen. Ohne eine Teilhabe am öffentlichen Leben und der dadurch kreierten persönlichen Identifikation bliebe ein emotionaler Bezug jedoch außen vor, sodass eine Tiefe an Erkenntnis und ein Urteilsvermögen über jeweilige Tragweiten verwehrt bliebe. Von Forschungsaufenthalt zu Forschungsaufenthalt reduzierte ich daher die Verwendung von PRA-Methoden zugunsten teilnehmender Beobachtung.

Abschließend betrachtet, steht Partizipation in dieser Arbeit nicht für eine einzelne Methode oder aber eine Forschungsmethodik im Sinne eines MethodenSets. Anstelle eines Werkzeugs, Forschung zu betreiben steht Partizipation viel ganzheitlicher für einen „way of life“ (Pratt 2003: 168), in welchem Forschungsinteressen in den alltäglichen Einstellungen und dem Verhalten des Praktizierenden aufgehen.

Dieses Kapitel diente der Offenlegung des Forschungsprozesses. Dies soll die Grundlage darstellen, die wissenschaftliche Qualität der vorliegenden Arbeit beurteilen zu können. Es wurde begründet, warum die Forschung eine persönliche und emotionale Note tragen darf und mitunter tragen soll. Auch im weiteren Verlauf der Arbeit sollen persönliche Perspektiven nicht verschleiert werden, sondern durchscheinen, um die Argumentationen mittels der Kenntnis dahinterliegender Motivationen bestmöglich verstehen und qualitativ einschätzen zu können. 



\section{B Theoretisch-konzeptioneller Rahmen}

In diesem Abschnitt werden die Vorüberlegungen der Einleitung anhand theoretischer Konzepte untermauert. Diese werden auf die Grundthematik der Arbeit soziale Sicherung im Zeichen gesellschaftlicher Transformation - so zurechtgeschnitten, dass sie einer erkenntnisgewinnversprechenden empirischen Anwendung im fidschianischen Kontext unterzogen werden können. Im methodologischen Abschnitt wurde die Bedeutung der eigenen Person herausgestellt. Auch in die Collage dieses Abschnittes fließt sie ein Stück weit mit ein. Idealerweise sind die verwendeten Konzepte, wie schon die Methodik, zum Teil der Entwicklungspraxis entsprungen, sodass die Erkenntnisse auf einen möglichen Anwendungsbezug hoffen lassen.

Die im weiteren Verlauf verwendete Terminologie entstammt natur- und sozialwissenschaftlichen Disziplinen sowie der praktischen Anwendung (Thywissen 2006: 448-52). Da diesen mitunter grundlegend verschiedene Weltanschauungen zugrunde liegen und zudem hauptsächlich in englischer Sprache diskutiert wird, führt dies zu Verständigungsproblemen. Durch Begriffserklärungen und sprachliche Genauigkeit schaffe ich im Folgenden Abhilfe und verdeutliche die eigene Perspektive. 



\section{Die Gabe - Einführung in kulturtheoretische Grundbegriffe}

Im Fokus dieser gesellschaftswissenschaftlichen Studie stehen Menschen. Sie werden einerseits als Individuen betrachtet, andererseits als Gruppen bzw. Teile von Gruppen. Aus humangeographischer Perspektive heraus interessiert in dieser Studie, wie Individuen über ihr Alltagshandeln kulturelle Gemeinschaften produzieren und reproduzieren, wie sich dies im Räumlichen widerspiegelt und wie sich Identitäten verorten lassen können. Zunächst führe ich daher in zentrale Begriffe der Studie ein.

\subsection{Relationales Raumverständnis}

Diese Studie arbeitet problemorientiert bzw. problemlösungsorientiert. Frei nach dem Motto „Der Raum hat keine Probleme!“ sind Menschen ins Zentrum der (human-)geographischen Analyse gerückt. Konsequenterweise stehen nicht Raumtheorien, sondern Gesellschaftstheorien im Fokus der Arbeit. Es wird nach gesellschaftlichen Zusammenhängen geschaut, welche auch anhand ihrer räumlichen Manifestierung begründet werden (Werlen 2008: 278-280). Räumliche Ordnungen sind jedoch mehr als ein Abbild gesellschaftlicher Strukturen. In der räumlichen Sphäre werden soziale Strukturen hergestellt bzw. Räume „produzieren“ gesellschaftliche Verhältnisse (Lefebvre 1972; Rothfuß 2012: 20f.). Räumliche Strukturen sind Ausdrücke menschlichen Handelns in historischer Dimension. Sie sind Produkte früherer Geschichte und Basis neuer Gesellschaftsformen in denen sich eine (scheinbare) Widersprüchlichkeit einerseits von Verstetigung akkumulierten menschlichen Handelns und andererseits von gesellchaftlichem Wandel widerspiegelt. Im Folgenden werden für diese Studie bedeutende gesellschaftliche Schlüsselbegriffe vorgestellt und in Relation zum Raum gesetzt.

\subsubsection{Gesellschaft und Kultur}

Der Begriff der Gesellschaft bezeichnet Menschen, die in einem sozialen Zusammenhang zu einander stehen. Diese Beziehung drückt sich in gemeinsamen Überzeugungen und Normen aus, welche durch Institutionen geregelt und aufrechterhalten werden. Über soziale Praktiken im Alltag wird der soziale Zusammenhang beständig reproduziert.

Die Gesellschaft steht dabei im Bezug zum Raum. Das Geflecht sozialer Beziehungen lässt sich räumlich verankern. Die Menschen nehmen den Raum wahr und grenzen Zugehöriges und Fremdes mehr oder weniger durchlässig voneinander ab. Beispielsweise in Form von Zäunen können Grenzen eine materielle Form im physischen Raum annehmen, häufiger jedoch haben Grenzen wie Staats- oder Gemeindegrenzen eine immaterielle Form, sind also im physischen Raum nicht sichtbar, sondern berufen sich auf gesellschaftliche Übereinkünfte. Räume sind Vorstellungswelten, denen soziale, politische, kulturelle oder ökonomische Eigen- 
schaften zugewiesen werden. Daher verschieben sich je nach zugrunde gelegter Kategorie die Muster, nach denen Räume strukturiert werden. Politische Grenzziehungen beispielsweise haben keine zwingende Aussagekraft über kulturelle, ökonomische oder sonstige gesellschaftliche Zusammenhänge. Stattdessen existieren vielfältige grenzüberschreitende Verbindungen und Bezüge. Lagebeziehungen beziehen sich daher nicht auf physische Distanzen, sondern drücken Wahrnehmungskategorien aus, zumal physische räumliche Distanzen mit fortschreitender Entwicklung von Kommunikations- und Transporttechnologien zeitlich betrachtet schnell überwunden werden können (Freytag 2014: 15-20).

Der Begriff der Kultur verweist auf die Symbol- und Sinndimension gesellschaftlicher Strukturen und Prozesse (Lossau 2014: 26). Gemeinschaften sind dabei kleine Einheiten einer Gesellschaft, innerhalb derer jeder jeden kennt, beispielsweise in Form von Dorfgemeinschaften. Während „Gesellschaft“ begrifflich übergeordnete Kollektive behandelt, durchmischen sich in der gelebten Praxis die Ebenen. Gemeinsame, die Individuen miteinander verbindenden Alltagserfahrungen erzeugen soziale Gemeinschaften, die abstrakt als kulturelle Gemeinschaften eine Gesellschaft bilden. Die gelebte Erfahrung akkumuliert sich und manifestiert sich symbolisch und materiell im Raum als Kulturlandschaft, und konstituiert sich im sozialen Miteinander über Werte, Traditionen und Rituale. Die Routinen und Narrative kennzeichnet eine Verstetigung, welche als kulturelle Muster verstanden werden können. Durch die Kultur wird so ein gemeinsamer Erfahrungs-, Erwartungs- und Handlungsraum gebildet, dessen Verbindlichkeit Vertrauen und Orientierung erzeugt und Zugehörigkeit bzw. Identität stiftet (Assmann 2002: 16). Identität entsteht also aus der Zugehörigkeit zu einer kulturellen Gruppe und lässt sich über die ihr zu Grunde liegende Raumvorstellung verorten. Mittels der imaginierten Verortung verleiht die kulturelle Identität gefühlte Stabilität und Sicherheit. Die imaginierten Räume und Identitäten konstituieren sich hierbei wechselseitig.

\subsubsection{Kulturelle Identität}

Assoziiert mit Begrifflichkeiten wie Vertrauen, Orientierung, Stabilität und Sicherheit ist die kulturelle Identität normativ betrachtet zunächst ein förderungswürdiges Gut. Vorsicht ist jedoch geboten, wenn solche Essentialismen zur Diskriminierung der Nichtmitglieder und zur Homogenisierung der zugehörigen Gruppe führen. Denn Kultur ist kein statisches Set von Denk- und Handlungsweisen, sondern ein aktiver, fortdauernder Prozess. Von einer geschlossenen Definition von Kultur wird daher abgesehen, da sie einengen würde und ihrer intrinsischen Pluralität nicht gerecht würde (Heidemann 2011: 18-20). Ebenso wenig stellen Räume ein Nebeneinander geschlossener Container dar und es ist trügerisch, Gesellschaften oder Gemeinschaften als homogene und statische Zusammenschlüsse zu begreifen. Die Dynamik und Komplexität wird deutlich, wenn man bedenkt, dass jedes Mitglied einer kulturellen Gemeinschaft seine gelebten Erfahrungen zwar vor einem gemeinsamen Erfahrungsschatz, jedoch individuell interpretiert 
(Giesen \& Seyfert 2013: 39). Ideen, Werte und Ideale werden vor dem Hintergrund normativer Ziele "guter" Kulturen, teils aktiv und bewusst, sowohl von Mitgliedern als auch Nicht-Mitgliedern der Gemeinschaft, gesteuert und verschoben. Kulturen unterliegen somit konfliktreichen, immerwährenden Veränderungsprozessen. Werte und Normen sind im Alltag innerhalb von Kulturen konflikthaft permanenten Aushandlungsprozessen unterworfen. Man kann entsprechend verschiedene Akteure und Strömungen innerhalb der Gemeinschaften ausmachen, die mit unterschiedlichem Erfolg ihre Ziele gesamtgemeinschaftlich oder gesellschaftlich durchzusetzen vermögen.

Derartige Strömungen lassen sich beispielsweise in „konservativ“, der Bewahrung des Bestehenden verschrieben, oder „progressiv“, dessen Veränderung suchend, einteilen. Individuen nehmen hierbei nicht ausschließlich eine Haltung ein, sondern können je nach Thema, sowie innerhalb eines Themengebietes, sowohl konservative als auch progressive Strömungen vertreten, sowie keine klare Meinung herausgebildet haben, entgegen ihrer Wertanschauung handeln, oder aber ihre Meinung ändern. So bilden sich innerhalb einer Kultur verschiedene Lebensweisen heraus, die als Subkulturen erfasst werden können. Einen einzelnen Menschen kennzeichnen hierbei plurale Zugehörigkeiten. In unterschiedlichen sozialen Kontexten nimmt man an vielseitigen Identitäten teil, ohne dass diese sich widersprechen müssen (Sen 2007: 33-35).

Weder Kulturen noch Identitäten, noch Strukturen, Systeme oder Traditionen werden in dieser Arbeit als statisch betrachtet. Struktur ist prozessual zu verstehen, als eine dynamische Entwicklung kultureller Kategorien und Beziehungen in einem System der Generierung und Regenerierung über Raum und Zeit (Sahlins 1981: 110f.) Tradition wird ebenfalls als ein Prozess verstanden. Dieser ermöglicht es über die Zeit, Veränderungen, den Wegfall oder die Hinzunahme von Verhaltensweisen als kulturelle Elemente in eine Gesellschaft zu integrieren, ohne dass damit ein Identitätsverlust einhergeht. Da verschiedene Identitäten sich nicht zwingend widersprechen, können auch aktuelle Wandlungen theoretisch nahezu widerspruchsfrei adaptiert werden (Sen 2007: 42f.). So konnte der christliche Glaube ein fester Bestandteil der fidschianischen Kultur werden, ohne ältere Bräuche zwingend zu verdrängen (Toren 1988: 697f.).

Neben solchen nichtkontrastierenden Identitäten gibt es auch kontrastierende. Die Auswahl ist dabei eigen- und fremdgesteuert. Wir entwickeln eigene Interessen, welche vom sozialen Umfeld beeinflusst werden. Die Stärke und Richtung der Beeinflussung ist dabei jedoch nur bedingt vorhersehbar: Wir können in ein privilegiertes Leben hineingeboren sein, dieses gutheißen und weiterführen, oder aber Ungerechtigkeiten entdecken und diesen entschieden entgegentreten. Manche Identitäten, wie die Staatsbürgerschaft, sind nicht frei wählbar, sondern werden einem aufoktruiert, jedoch kann man zu einem gewissen Grade entscheiden, wie wichtig diese Identität für einen ist (Sen 2007: 38-43).

Menschen schließen sich entsprechend ihrer Zugehörigkeiten zu Gruppen zusammen. Die Individuen gehören zeitgleich mit unterschiedlicher Stärke verschie- 
denen Subkulturen an und grenzen sich von anderen ab bzw. werden ausgegrenzt. Manche Subkulturen definieren sich bewusst als Gegenkultur zum sogenannten Mainstream. Konflikte entstehen dabei in und zwischen den Gruppen. Konflikt wird in dieser Arbeit daher als sozialer Fakt definiert. Konflikte von Menschen über Werte und entsprechend dieser ausgeführte oder ausgebliebene Handlungen sind elementarer Bestandteil menschlichen Zusammenlebens. Daher ist Konflikt nicht negativ besetzt, wohl aber kann es bei Versuchen der Konfliktlösung zu ethisch verwerflichen Handlungen kommen. So wie es einer Kultur zur Herausbildung gesellschaftlicher Werte bedarf, bedarf es einer Konfliktkultur zum adäquaten Umgang mit Konfliktkonstellationen.

Weder lassen sich alle Menschen klar und ausschließlich einer bestimmten Gruppe innerhalb einer Kultur zuordnen, noch sind scharfe Trennlinien zwischen verschiedenen Kulturen stets einwandfrei zu zeichnen. Da sich Normen und Werte sowie die daraus abgeleiteten Lebensweisen auf einem Kontinuum zwischen Extrempositionen bewegen, beispielsweise zwischen den vermeintlichen Gegenpositionen individualistischen und gemeinschaftlichen Verhaltens, Egoismus und Altruismus, oder Freiheit und Kontrolle, und konstant neu ausgehandelt werden und sich verschieben, ist eine disjunkte Klassifizierung müßig. Umgekehrt gibt es jedoch sehr wohl Menschen, deren gelebte Erfahrungswelt, deren Werte und Normen und daran orientierten Lebensweisen größere Gemeinsamkeiten aufweisen als zu anderen Menschen, sodass der Kulturbegriff keinesfalls obsolet ist, es jedoch zu kulturellen Überlappungen kommt. Von anderen Kulturen unbeeinflusste Gesellschaften sind auf unserer Erde kaum auffindbar, sodass Kulturen als Hybride verstanden werden können. Wenn wir uns die Geschichte Fidschis anschauen, verortete Abel Tasman im Jahre 1643 die Fidschi-Inseln für Europäer auf Karten, 1774 setzte James Cook als wohl erster Europäer seinen Fuß auf fidschianisches Gebiet, 1835 gründete der erste christliche Missionar eine Mission und 1874 wurde Fidschi britische Kolonie. Alle Eckdaten könnten als Beginn der Hybridisierung der fidschianischen Kultur gesehen werden. Andererseits wurden die Fidschi-Inseln von Nachbarterritorien aus erstbesiedelt und standen seither in Kontakt zu weiteren pazifischen Inselstaaten, sodass eine „Reinkultur“ stets Imagination ist.

\subsubsection{Transkulturalität statt Multikulturalität}

Disjunkte Verortungen von Kulturräumen sind nicht möglich, für Orte der Überschneidung hat der Begriff der Multikulturalität Einzug erhalten. In der Migrationsforschung haben sich für multikulturelle Orte die Metaphern der Salatschüssel oder der Pizza (Zutaten behalten ihren Eigengeschmack, erzeugen jedoch nur in Kombination die Lieblingsspeise) etabliert (Heidemann 2011: 220f.). Diese zeigen einerseits das eigenständige Nebeneinander, andererseits im Zusammenspiel die Bereicherung für die Gesellschaft. Normativ soll Multikulturalität das friedliche Zusammenleben fördern. Praktisch wirkt der Begriff jedoch trennend, polarisie- 
rend, da die Eigenständigkeit und Unterschiedlichkeit einzelner kultureller Bestrebungen sich manifestiert, eine Statik suggeriert wird und Gemeinsamkeiten verschleiert werden (Sen 2007: 174; Aigner 2013: 21-23). Mögliche Konflikte werden dann zwischen den Kulturen verortet und „Lösungen“ werden als Bevor- bzw. Benachteiligungen antipiziert, sodass sich Konfliktpotentiale verschärfen.

Der Begriff der Transkulturalität hingegen, zu dem die Methaper des Schmelztiegels passt, zielt darauf ab, dass Kulturen ineinander aufgehen. Für die gesellschaftliche Übernahme und Adaption bestimmter kultureller Praktiken und ihrer Rekontextualisierung im neuen sozio-kulturellen Umfeld birgt ein Verständnis von Prozessen einer Transkulturation Erklärungen. Das Phänomen ist bisher jedoch aus wissenschaftlicher Sicht wenig beleuchtet; Transkulturationskonzepte haben aber gerade in ozeanischen Gesellschaften aufgrund ihrer kulturellen Vernetzung ein enormes Potential, zumal die Gesellschaften selbst ihre kulturellen Austausche verinnerlichen und über diese reflektieren (Hermann 2007: 257-259). Der Begründer des Konzeptes der Transkulturation, Fernando Ortiz, schreibt, dass der Prozess zwingend zu einem Verlust oder einer Entwurzelung der vorherigen Kultur führe (1947/1995: 102). Ich möchte jedoch mit diesem Begriff Hoffnungen hervorheben, dass das Zusammenspiel verschiedener kultureller Strömungen an einem Orte alle Beteiligten bereichert. Die transkulturelle Gesellschaft zeichne sich demnach nicht durch einen Verlust ihrer Historie und kultureller Identitäten aus. Stattdessen kennzeichne die Mitglieder ein breiter, gemeinsamer kultureller und historischer Schatz an Erfahrungen und Werten. Dieser Auffassung zufolge durchdringen und befruchten sich die Kulturen bereits gegenseitig, eine künstliche Trennung ist weder möglich noch wünschenswert. Friedvolle Lösungen möglicher Konfliktkonstellationen können angestrebt werden, die sowohl die Gesellschaft insgesamt als auch ihre Individuen tragen können.

Für ein umfassendes Verständnis von Transkulturalität bedarf es nicht nur einer Untersuchung des Zusammenspiels verschiedener Kulturen an nur einem Ort. Zur transkulturellen, gegenseitigen Einflußnahme gehören ebenso die Wirkungen, die auf die Ursprungsregionen zurückstrahlen. Räumlich basiert diese Studie jedoch auf einer lokal begrenzten Untersuchung fidschianischer Küstendorfbewohner. Einer umfassenden Erforschung von Transkulturalität möchte diese Arbeit daher nicht hinreichend gerecht werden, da der Fokus einseitig auf die Beeinflussung der fidschianischen Kultur gelegt wird. Wie umgekehrt fidschianische kulturelle Konzepte Wirkungen auf andere Kulturen ausüben, liegt nicht im Zentrum des Interesses dieser Arbeit. Im empirischen Abschnitt begnüge ich mich daher vorwiegend, der Hybridität der fidschianischen Gesellschaft und ihrer Bedeutung auf gutes Leben nachzugehen.

\subsubsection{Transdisziplinäre Erfassung des Wertefundaments kollektiver Identitäten}

Insbesondere die Erfassung, Definition und Abgrenzung einer Gesellschaft gestaltet sich als schwierig. Ihr wird allgemein eine kollektive Identität zugesprochen, 
die eine Ähnlichkeit im Wertefundament der Angehörigen gegenüber Außenstehenden suggeriert. Dennoch gibt es keinen absoluten Wertekonsens, selbst die Formulierung eines Minimalkonsenses würde stets streitbar sein. Begriffe wie Freiheit, Solidarität, Teilhabe und Gerechtigkeit besitzen keinen statischen Bedeutungsinhalt und bedürfen daher stetiger, konflikthafter Aushandlungsprozesse. Kollektive Identitäten sind daher von vagem, unaufhebbar uneindeutigem und intransparentem Charakter, in deren Zentrum ein ,leerer Signifikant" steht (GieBen \& Seyfert 2013: 39-43; Karakayali 2013: 21). Diese signifikante Leere ist paradoxerweise jedoch nicht belanglos, sondern erzwingt ein unaufhörliches Wiedererzählen bei individuellem Neuauslegen unter den jeweils aktuellen Begebenheiten, die dem Signifikanten Stabilität und der jeweiligen Gesellschaft ihre Identität verleiht.

Versuche, den Wertekodex einer Kultur zu beschreiben, sind daher zwingend unvollständig und verzerrt. Um das Undarstellbare dennoch (zumindest ansatzweise) fassen zu können, bedarf es eines Rückgriffs auf darstellbare Indikatoren der Kollektiven Identität (Gießen \& Seyfert 2013: 39f.). Im Bezug zum gelebten Alltag stehende Ursprungsmythen und Legenden, Rituale und Lieder, Ikonen und Denkmäler werden in dieser Arbeit daher zur Erfassung einer postulierten kollektiven Identität der fidschianischen Kultur zu Rate gezogen - und in Gesprächen mit den Dorfbewohnern gemeinsam interpretiert. Hierbei tritt eine Bedeutung des Spirituellen, von Zauber und Magie zu tage. Über den freien Einbezug dieses Emotionalen, Nicht-Quantifizierbaren und wissenschaftlich nicht verortbaren mittels der Beteiligung wissenschaftsexterner Akteure (Bogner et al. 2010: 13f.) erhält die Studie somit transdisziplinäre Dimensionen. ${ }^{17}$

\subsubsection{Soziales Handeln}

Menschliches Handeln bezieht sich auf die Fähigkeit, unabhängig Entscheidungen trefen zu können und struktuelle Bedingungen ändern zu können. Dieses Verständnis sieht Menschen als aktive und bewusste Gestalter ihrers Lebensraumes an und nicht als passiv reagierend auf externe Einflüsse, wie es statische Modelle menschlichen Verhaltens suggerieren (Castles et al. 2014: 31; Werlen 2008: 278290, 356). Der Begriff des Sozialen hat in der Wissenschafts- wie in der Alltagssprache verschiedenste Konnotationen. Auf einer rein beschreibenden Ebene wird jede menschliche Interaktion als soziale Interaktion gewertet. Weiterhin hat jede menschliche Handlung, sowie auch jede unterlassene Handlung, Konsequen-

\footnotetext{
${ }^{17}$ An meinem Verständnis von der Aufklärung, speziell von der Entstehung des westlichen Wertegerüstes mit den Stützpfeilern Freiheit, Gerechtigkeit und Demokratie hat Amartya Sen großen Anteil. Ich finde es erstaunlich, dass mir ein Inder die Exegese meines eigenen Werteverständnisses besser veranschaulichen kann als europäische Philosophen. Ich bin dankbar und beeindruckt, wie Sen meine leeren Signifikanten historisch auslegt und mit Inhalt füllt und so - trotz weitreichender Kritik - insgesamt meine Identifikation mit meiner kulturellen Herkunft stärkt. Ohne mir Anmaßen zu wollen, jemals die Qualitäten Amartya Sens erreichen zu können, stimmt mich diese Erkenntnis jedoch positiv, sinnbringend von Werten in der Kultur Fidschis erzählen zu dürfen.
} 
zen - sie ist also von zwischenmenschlichem Charakter, stellt eine Interaktion dar. Ergo ist alles, was Mensch tut - oder nicht tut - sozial. Um von dieser allumfassenden Grunddefinition des Sozialen zu einem für diese Arbeit handhabbaren Konzept zu gelangen, wird diese heruntergebrochen.

Wenn über verschiedene Gruppen innerhalb einer Gesellschaft gesprochen wird, wird oft von „sozialen“ Kategorien gesprochen, die Gruppen voneinander abzugrenzen. Soziale Gruppen können sich demnach beispielsweise nach ethnischer oder religiöser Zugehörigkeit, politischer Gesinnung, Alter oder Geschlecht unterscheiden. Man hat Akteursgruppen bzw. methodologisch Fokusgruppen im Sinne, deren Mitglieder wesentliche Einstellungsmerkmale miteinander teilen. Karten stellen oftmals die räumliche Verteilung von Menschen entsprechend sozialer Kategorien dar.

Diese humangeographische Studie möchte jedoch das Soziale in erster Linie als ein besonderes Charakteristikum menschlichen Handelns verstehen. Das heißt, diese Arbeit ist menschzentriert und legt dabei ein besonderes Augenmerk auf räumliche Dimensionen menschlichen Handelns und akkumulierter menschlicher Handlung. Soziales Handeln wird daher zunächst künstlich von sonstigem menschlichen Handeln getrennt behandelt, um das Soziale für diese Arbeit handhabbar zu machen.

Mitunter werden die Worte „sozial“ und gesellschaftlich“ als Synonyme verstanden, in der Wissenschaft spricht man von Sozial- bzw. Gesellschaftswissenschaften. Häufiger wird jedoch das Soziale als eine Unterkategorie des Gesellschaftlichen gesehen. Eine gängige, künstliche Untergliederung des Gesellschaftlichen ist die Einteilung in „sozial“, „ökonomisch“ und „politisch“. So haben sich Soziologie, Wirtschaftswissenschaften und Politikwissenschaften als weitestgehend eigenständige wissenschaftliche Disziplinen entwickelt. Jeweilige Teilgebiete wie Wirtschaftssoziologie, Wirtschaftspolitik und Sozialpolitik zeugen jedoch von der Verwandtschaft, die auch heute noch den Disziplinen anhaftet.

In der westlichen Welt haben sich die Unterscheidungen nach sozial, politisch und wirtschaftlich so stark verselbstständigt und veralltäglicht, dass die Künstlichkeit der Trennung kaum reflektiert wird und die Trennung als Fakt akzeptiert ist. Über die letzten Jahrhunderte haben sich Idealtypen herauskristallisiert, was unter sozialem Handeln im Gegensatz zu wirtschaftlichem und politischem Handeln zu verstehen sei. Wirtschaftliches Handeln wird in den Wirtschaftswissenschaften vielfach als „,rationales“ Handeln deklariert. Ihr Idealtypus, der bomo oeconomicus, zielt demnach ausschließlich auf die Maximierung des persönlichen Nutzens ab (Rogall 2013: 110f.; Sen 2010: 206). In neoklassischen Grundmodellen hält monetär ausdrückbarer Reichtum als bester Indikator für persönliches Wohlbefinden her und vollständige Information wird vorausgesetzt. Genuin politisches Handeln wird hingegen von den Politikwissenschaften meist machttheoretisch und konfliktorientiert angelegt. Es zielt demzufolge auf die Mehrung oder den Erhalt von Macht oder die Beeinflussung von Machtverhältnissen ab, um Konflikte zu regeln, wobei Kampf und Gewalt probate Mittel darstellen (Macchiavelli ca. 1513/2004; 
Rüb 2014: 362-365). Soziales Handeln hingegen zielt in dieser Sichtweise auf zwischenmenschlichen Ausgleich von Macht und Reichtum ab, basierend auf normativen Gerechtigkeitsverständnissen. Hier steht nicht der individuelle Nutzen, sondern altruistisch der gesellschaftliche Nutzen im Vordergrund.

Soziales Handeln kann daher im Widerspruch zu rationalem oder politischem Handeln stehen. Wirtschaft und Politik seien im Kern unsozial und damit normativ negativ besetzt. Streng rationales Handeln sei zwingend egoistisch und „das Politische [als] ein anderer Name für Konflikt, Macht, Widerstand, Dominanz und Unterordnung" (Marchart 2008: 16) zu verstehen. Menschliches Handeln entspricht jedoch nach Ansicht dieser Arbeit in der Praxis keiner dieser Reinformen. Ich zeige auf, dass ein Hochhalten solch künstlicher Trennungen menschlichen Handelns in politische, wirtschaftliche und soziale Sphären die Gesellschaft entzweit.

Das abendländliche Verständnis von Politik als Herrschaft und seiner impliziten Gleichsetzung von Politik, Kampf und Gewalt stützt sich auf die modernen Politikbegriffe, wie sie beispielsweise Max Weber prägte. Er schreibt, „daß der moderne Staat ein anstaltsmäßiger Herrschaftsverband ist, der innerhalb eines Gebietes die legitime physische Gewaltsamkeit als Mittel der Herrschaft zu monopolisieren mit Erfolg getrachtet hat" (Weber 1919: 402) und der Kampfbegriff zieht sich als Wesensmerkmal politischen Handelns durch seine Schriften (Palonen 1998: 167). Auch nach Karl Mannheims Politikverständnis sind „die Machtkompetenzen im staatlichen wie im zwischenstaatlichen Leben (...) im irrationalen Kampfe errungen“" (Mannheim 1929/1952: S. 100, zitiert nach Rüb 2014: 370).

Hannah Arendt bricht mit diesem Politikverständnis. In ihrer Perspektive des Handelns basiert Politik auf menschlicher Pluralität. Politisches Handeln liegt in der Beziehung zwischen Menschen und ist offen und horizontal angelegt (Heuer 2006: 8f.). Es ist demnach genuin zwischenmenschlich und sozial in dem Sinne, dass es der Erreichung eines gesellschaftlichen Wertes abzielt. Freiheit ist der Sinn des Politischen. Menschen können nur in Bezug aufeinander frei sein, nicht in Isolation. Abseits eines Raumes der Freiheit menschlicher Pluralität gibt es keine Politik im eigentlichen Sinne (Arendt 1959/2000: 201f.; Arendt 1993: 52). Das politische Handeln zeigt sich in ,der menschlichen Fähigkeit [...] sich mit anderen zusammenzuschließen und im Einvernehmen mit ihnen zu handeln" (Arendt 1970: 12). Während man über Kooperation zu Macht als Bestandteil politischen Handelns und Basis zur Übernahme von Verantwortung kommt, stellt Gewalt das Gegenteil von Macht dar, da sie den Menschen ihrer Freiheit und Souveränität beraubt und die Gesellschaft apolitisiert (Heuer 2006: 11f.). Diese Studie orientiert sich an solchen Sichtweisen einer Verbundenheit der politischen und sozialen Sphäre. Denn, dem Begriff „Politik“ ein Freund-Feind-Bild (Schmitt 1932/1963: 26) zu Grunde zu legen, in dem es um Macht als Gewaltherrschaft und Unterdrückung geht, trägt zur Entzweiung menschlicher Gesellschaften bei. Dass derartige Dichotomien menschlichen Handelns gerade in dem außereuropäischen Kontext dieser Arbeit nur bedingt hilfreich sind, möchte ich insbesondere hinsichtlich des 
Begriffspaares „sozial“ und „wirtschaftlich“ am Beispiel der „Gabe“ verdeutlichen.

\subsection{Die Praxis der Gabe}

Marcell Mauss Studie über „Die Gabe“ (1923-25/1990) ist ein Klassiker der soziologischen und ethnologischen Forschung. Für die vorliegende Studie zuträglich, basiert Mauss Analyse auf einen Vergleich pazifischer Kulturen. ${ }^{18}$ Auch wenn Mauss das Prinzip der Gabe als vormodern bezeichnet, werde ich im Verlauf der Arbeit das Phänomen als bedeutende Komponente gesellschaftlicher Interaktion im heutigen Fidschi herausarbeiten.

Das banal anmutende Geben eines materiellen Gegenstandes steht im Zentrum der Untersuchung: Jemand gibt jemand anderem etwas. In einem engeren ökonomischen Sinne handelt es sich um die freiwillige Fortgabe eines Besitztums. Geht man vom Nutzenbegriff gemäß der Wirtschaftstheorie rationaler Wahl ${ }^{19}$ aus, so ist die Handlung zunächst unlogisch, da sie keine Gegenleistung fordert; der eigene Besitz, das eigene Wohlbefinden wird geschmälert. Sie steht damit in direktem Gegensatz zum ökonomischen Prinzip des Eigennutzenmehrens. Dieser Besitzwechsel, ein ökonomisch klar fassbarer Vorgang, ist rational nicht zu erklären. Genauso wenig lässt sich die Gabe politisch erklären, da mit der Abgabe von Besitz kein Machtgewinn oder Macherhalt einhergehen muss, das Gegenteil wahrscheinlicher erscheint. Auch orientiert sich die Gabe nicht sozial nach Gerechtigkeitsprinzipien wie Verteilungsgerechtigkeit, dass sich die Gabe fair nach Maximen wie Bedürftigkeit, Nutzen oder Gleichheit ausrichten würde.

Dass Gaben dennoch einen persönlichen Nutzen zu haben scheinen, zeigt beispielsweise folgende immaterielle Gabe: Werden wir von einem Fremden nach dem Weg zum Bahnhof gefragt, helfen wir gerne. Die Auskunft kostet im mindesten Zeit und eine Gegenleistung vom Gegenüber ist nicht zu erwarten, auch unser eigenes soziales Umfeld wird nicht davon erfahren, sodass wir dort nicht an Achtung gewinnen; und dennoch erfreuen wir uns an unserer guten Tat. Jedoch sind wir keine bloßen Altruisten, sondern erhoffen diese soziale Praxis der Hilfe bei

\footnotetext{
${ }^{18}$ Trotz der mitunter einem kolonialen, imperialistischen und - nach heutigen Maßstäben - rassistischen Sprachgebrach entnommenen Charakterisierungen als „primitive“, „,niedere“, ,rückständige“ oder ,archaische“ Gesellschaft (Mauss 1923-25/1990: 20f.), zeugen Mauss“ Analysen von kultureller Empathie und ihre Tiefe und Klarheit findet bis heute Würdi $\neg$ gung (Adloff \& Mau 2005: 10). ${ }^{19}$ Auch wenn „rational“ gerne mit „,vernünftig“ gleichgesetzt wird, so wird das Wort hier im ökonomischen Sinne der Rational Choice Theorie gebraucht. Kluge Maximierung des Eigennutzens deren Definition von Rationalität - zeugt jedoch von einem extrem beschränkten Verständnis von Vernunft. Auch wenn Eltern sich besser fühlen, wenn sie ihre Kinder aufziehen, so kann Eigennutz allein nicht die Liebe und damit verbundenen umsorgenden Gesten erklären. Genauso kann der Kampf gegen Ungerechtigkeiten nicht generell mit persönlichem Streben nach privatem Gewinn erklärt werden, dass Personen einzig ihren eigenen Schmerz beim Anblick von Ungerechtigkeit lindern wollen. Rationalität ist nicht zwingend vernünftig und eine Annahme, Menschen würden rational Handeln, ist schon gar nicht vernünftig, da Gewohnheit, aber auch Leidenschaft und Impulsivität das intelligente Verfolgen des Selbstinteresses konterkarieren (Sen 2010: 203-211).
} 
eigener Desorientierung in fremden Städten. Hierbei wird die Betrachtungsebene einer Transaktion zwischen zwei direkt beteiligten Personen ausgeweitet auf die Gesellschaft.

Mauss zeigt in seiner Studie die weiteren gesellschaftlichen Implikationen des Warentransfers auf und interpretiert die Geste als totales, gesellschaftliches Phänomen (1923-25/1990: 17). Er weist ihr so eine Handlungslogik nach. Das theoretisch freiwillige Geschenk ist ihm zufolge ein komplexes Phänomen, in dem gleichzeitig alle Arten von Institutionen zum Ausdruck kommen, - ökonomische, religiöse, mythologische, rechtliche, moralische, ästhetische und soziomorphologische, - dem im Ergebnis ein zwanghafter und eigennütziger Charakter innewohnt (Evans-Pritchard 1990: 10; Mauss 1923-25/1990: 17f.). So erfolgt eine Gabe nicht wertneutral zwischen zwei Personen, sondern ist in ein gesellschaftliches System eingebettet (Hedtke 2014: 60f.). Die jeweiligen Personen vertreten Kollektive - sei es eine Familie oder ein Klan - und sind als Repräsentant moralische Instanz. Jeder materiellen Gabe folgt eine immaterielle Gegengabe: Der Beschenkte erwidert die Leistung im Mindesten ritualisiert oder in Form von Höflichkeiten; er erkennt durch die Annahme die Leistung an. Der Gebende erhält Prestige und Ehre innerhalb seines Kollektivs und - sofern nicht identisch - des Kollektivs des Beschenkten, sodass eine Gegenleistung erbracht wird (Mauss 1923-25/1990: 22). Da die Nichtannahme ein Akt der Verweigerung der Zugehörigkeit darstellen würde, der einer „Kriegserklärung“ (Mauss 1923-25/1990: 37) gleichkäme, besteht ein Zwang zur Annahme. Abgesehen von Gaben als Almosen aus Motiven der Nächstenliebe, geht zudem oftmals eine materielle Gegengabe einher. Menschen, die einen beschenken und einem somit offensichtlich wohlgesonnen sind, ist man im Regelfall ebenfalls wohlgesonnen. Nicht nur aus Pflichtgefühl, sondern vor allem aus dem Wunsch, ebenfalls etwas Gutes zu leisten, erfolgt eine Gegengabe. Dieses Prinzip der Gegenseitigkeit heißt Reziprozität. Selbst Almosen werden vom Empfänger mit Dankbarkeit entgegengenommen, eine persönliche Beziehung mit immateriellem Rückfluss wird aufgebaut, sodass eine Gabe ohne Rückgabe, ein anonymisiertes Geschenk wohl allenfalls in der Theorie existieren kann (Eisenstein 2011: 8).

\subsection{Gabenökonomie als Wirtschaftsweise}

Allgemein ist es hinderlich, sich die Gabe als Tauschhandel vorzustellen. Gaben sind in erster Linie kein lineares Phänomen, kein abgeschlossener Handel zwischen Individuen. Sie zirkulieren (Eisenstein 2011: 6). Die Gabe stellt lediglich eine Momentaufnahme in einem größeren System des Gebens dar. Dies stellt Mauss besonders beindruckend beim von Bronisław Malinowski beschriebenen Kula-Handel auf den Trobriand-Inseln des heutigen Papua-Neuguinea fest (Mauss 1923-25/1990: 44f., 53-73): Gaben sind über Inseln und Stämme hinweg in einem System des Gabentauschs eingebettet, welches das gesamte wirtschaftliche und soziale Leben durchdringt. Halsketten werden kreisförmig von Insel zu Insel wei- 
terverschenkt, Armreife wandern entgegengesetzt, alles findet sich beständig im Umlauf.

Das ganze Stammesleben ist durchdrungen von einem beständigen Geben und Nebmen; jede Zeremonie, jede legale und konventionelle Handlung wird von materiellen Gaben und Gegengaben begleitet; der gegebene und genommene Reichtum ist eines der Hauptinstrumente der sozialen Organisation, der Macht der Häuptlinge, der Bande der Blutsoder Heiratsverwandschaft. (Malinowski 1922: 167, zitiert nach Mauss 192325/1990: 70)

Wenn eigene Gaben keine direkte Gegengabe implizieren, sondern sich Kreisläufe des Gebens bilden, bekommt man seine Gaben höchst indirekt und zeitversetzt zurück, sodass die einzelne Gabe als Geschenk aufgefasst werden kann und die Geste eine moralische Aufwertung erhält. Reziprozität verläuft nicht nur direkt oder kreisförmig, sondern kann auch intergenerationelle Phänomene beschreiben. Die Fürsorge, die wir von unseren Eltern bekommen haben und vor allem das „Geschenk“ des Lebens, können wir ihnen niemals gleichwertig zurückgeben, wohl aber können wir uns um die eigenen Kinder bzw. allgemein um die nachfolgenden Generationen kümmern.

Den eigentlichen Wert einer Gabe stellt nicht der materielle Gegenstand dar, ihren Wert erhält sie durch die gebende Person und wird damit „beseelt“. Aufgrund der persönlichen Beziehung, innerhalb welcher der Gegenstand abgetreten wird, ist dieser nicht leblos, sondern ein Teil vom Geber (Därmann 2010: 16f; Mauss 1923-25/1990: 32-34). Geschenke werden nicht nur aufgrund ihres praktischen Nutzens geschätzt, über die Geste des Schenkens entwickeln wir einen persönlichen Bezug zum Gegenstand bzw. zur Person, die sie uns gibt. Damit ist der profane Gegenstand nicht beliebig austauschbar, sondern einzigartig. Das Unikat besitzt soziale und historische Dimensionen. Aufgrund seiner Einzigartigkeit und Individualität ist es nicht vergleichbar, unersetzbar und bei Verlust nicht zu kompensieren. Finanzielle Werte beruhen auf Vergleich, geschenkte Gegenstände sind einmalig und somit nicht vergleichbar. Sie sind jenseits weltlicher Wertmaßstäbe wie Preiskategorien und damit letztlich unendlich wertvoll bzw. heilig. Folgende westafrikanische Parabel verdeutlicht anschaulich, wie ein Gegenstand über die Weitergabe als Geschenk eine persönliche, immaterielle Komponente bekommt und ihm eine immaterielle, einzigartige Seele eingehaucht wird.

Ein Junge möchte seiner Lehrerin ein Geschenk machen. Da er kein Geld hat, kann er ihr nichts kaufen. Also beschließt er, einen mehrstündigen Fußmarsch zum Meer zu tätigen. Dort packt er etwas Sand in ein Gefäß und schenkt dieses am nächsten Schultag der Lehrerin. Diese wundert sich und sagt: „Das Meer ist aber sebr weit weg von hier." Der Junge antwortet: „Frau Lehrerin, der Weg ist Teil des Geschenks. “

Betrachtet man nun nicht einzelne Gaben als losgelöst voneinander, bildet sich über den reziproken Gabentausch ein sozio-ökonomisches System, die Gaben- 
ökonomie. Dieses System ist dabei nicht rein wirtschaftlicher Natur, sondern durchdringt alle Sphären menschlichen Handelns.

Gerade vor dem Hintergrund der aktuellen weltweiten Wirtschafts- und Finanzkrisen finden alternative Wirtschaftsmodelle auch in Europa Zulauf. ${ }^{20}$ Die Gabenökonomie wird dann als Gegenentwurf zur Marktwirtschaft interpretiert. Marktwirtschaft wird diesen Darstellungen zufolge zumeist auf neoliberale, kapitalistische Ideale beschränkt. ${ }^{21}$ Denen wiederum liegt das Konzept selbstregulierender Märkte zugrunde, welches Nutzen- bzw. Wohlstandsmaximierung garantieren soll. Deregulierung, Privatisierung und Liberalisierung von Märkten seien dann die Kernprinzipien, mit denen der Idealzustand der Wohlfahrt für alle erreicht werden könne. Weiterhin entspräche dem marktwirtschaftlichen Ideal eine entpersönlichte Beziehung, ein kommerzieller Tausch losgelöst von allen sonstigen Beziehungen. Marktwirtschaft ist dann eine Wirtschaftsweise, die auf die Maximierung des persönlichen Nutzens ausgelegt ist und Aushandlungsprozesse bedarf, in denen beide Seiten einen maximal möglichen Gewinn auf Kosten des anderen anstreben. Nach vereinfachten, idealisierten Wirtschaftsmodellen erzielen dabei immer beide Seiten ein mehr an Nutzen, da sonst kein Handel zustande käme (Varian 2004: 295). Durch rein wirtschaftliche, unpersönliche Beziehungen bleibt der Nutzen beidseitig, zudem ermöglichen sie einen zeit- und kostenextensiven Austausch (Rogall 2013: 77). Konkurrenz, möglichst vollständige, ist ein weiteres Ideal des Kapitalismus, welches in der Theorie optimale, das heißt geldmaximierende, Wirtschaftsbedingungen verspricht (Varian 2004: 14-17, Rogall 2013: 74f., 80).

Auf Basis solcher Darstellungen von Marktwirtschaft setzen Fundamentalkritiken an. Bei ungleichen Startvoraussetzungen wie ungleichem Markt- und Informationszugang (Varian 2004: 686-704) neigt dieses System zur Ausbeutung und Unterdrückung, in der sich auf Kosten anderer bereichert wird, sodass sich de facto Gewinner und Verlierer herauskristallisieren (Peet \& Hartwick 2009: 166-172). Ozeanische Länder könnten in internationalen Wirtschaftsbeziehungen aufgrund ihrer infrastrukturellen, demographischen und industriellen Ausgangslage zu letzteren gehören.

Damit ein kommerzieller, entpersönlichter Tausch zustande kommen kann, müssen Waren einen finiten Wert besitzen. Das Absprechen der Einzigartigkeit und Heiligkeit eines Gegenstandes erreicht man über Standardisierung und Ent-

\footnotetext{
${ }^{20}$ Insbesondere verbunden mit den Begriffen „Postwachstum“ und „Degrowth“ entstehen weltweit Bewegungen, die alternative Wirtschaftsweisen erforschen, propagieren und praktisch umzusetzen versuchen (Eisenstein 2011, Schmelzer 2014). Auch die Deutsche Forschungsgemeinschaft fördert seit 2011 ein wissenschaftliches Kolleg zur Erforschung von künftigen Postwachstumsgesellschaften (DFG 2014).

${ }^{21}$ Der Kalte Krieg schuf demnach einen Nährboden für plakative, simplizistische und ideell aufgeladene Darstellungen des Gegensatzpaares Kapitalismus und Sozialismus bzw. Markt- und Planwirtschaft. Der Zusammenbruch der planwirtschaftlichen Sowjetunion steigerte die Wirkmächtigkeit des Neoliberalismus und erlangte u.a. durch die Interventionen von IMF und Weltbank im Sinne des Washington-Consensus weltweit Vormachtstellung (Block 2001: xx; Rogall 2013: 101-108).
} 
persönlichung der Ware (Eisenstein 2011: 37-39). Somit könne kapitalistische Marktwirtschaft im Vergleich zur Gabenökonomie kein gesamtgesellschaftliches und individuelles Maximum an Wohlstand hervorbringen, da die Standardisierung und Verpreislichung von Waren ihren Wert mindert. ${ }^{22}$ Für die Fidschi-Inseln ist anzunehmen, dass sie als hybride Gesellschaft mit beiden Systemen bekannt sind und die jeweiligen Vor- und Nachteile Auswirkungen auf ihr „gutes Leben“ haben.

Der Mensch wird in dieser Arbeit als soziales Wesen aufgefasst, bzw. im Sinne menschlicher Pluralität (Arendt 1959/2000: 201f.; Arendt 1993: 52; Heuer 2006: 8f.). werden Menschen als soziale Wesen aufgefasst. Zwischenmenschlichkeit stellt nicht nur eine Bereicherung für alle Beteiligten dar, sondern ist essentiell für menschliches Leben. Rein marktwirtschaftliche Interaktionen verwerfen dieses Element. Eine künstliche Entzweiung von Wirtschaft und Gesellschaft wird in dieser Arbeit daher als Versuch der Entmenschlichung aufgefasst und bildet die Hauptkritik an kapitalistischer Marktwirtschaft. Mit der Verdrängung menschlicher Beziehungen durch Dienstleistungen verfällt menschliches Leben.

Ähnlich formuliert auch Karl Polanyis, dessen Erkenntnissen ich mich anschließen möchte, dass menschliche Wirtschaftsweisen in sozialen Beziehungen eintauchen: Menschen handeln nicht wirtschaftlich, um in den Besitz materieller Güter zu kommen, sondern zur Sicherung und Verbesserung ihres sozialen Status, ihrer sozialen Ansprüche und Zugänge. Sie schätzen materielle Güter nur, wenn diese ihrer nicht-ökonomischen Motive als nützlich erscheinen (1944/2001: 48). Entpersönlichte Wirtschaftsbeziehungen können daher kein menschliches Ideal darstellen. Diese Arbeit folgt dem Verständnis, die Wirtschaft sei in die Gesellschaft eingebettet. Die Entzweiung von Wirtschaft und Gesellschaft ist eine nicht zu erreichende Utopie des Kapitalismus:

Our thesis is that the idea of a self-adjusting market implied a stark utopia. Such an institution could not exist for any length of time without annibilating the buman and natural substance of society; it would have physically destroyed man and transformed his surroundings into a wilderness. (Polanyi 1944/2001: 3)

\subsection{Soziale Beziehungen und Sozialkapital}

Eine strikte Trennung menschlichen Handelns in wirtschaftlich, politisch und sozial ist für diese Studie weder möglich noch erstrebenswert. Dennoch können

\footnotetext{
22 Dieser Absatz soll nicht als technologiefeindlich verstanden werden. Technischer Fortschritt ermöglicht und beschleunigt kulturelle Erzeugnisse und einbringliche interkulturelle Austausche; er kann Lebensqualität und Wohlbefinden erhöhen. Massenproduktion und Skaleneffekte erhöhen ungemein die Produktivität des Einzelnen. Nichtsdestotrotz wird das produzierte Einzelstück beliebig austauschbar und Menschen verlieren den persönlichen Bezug zu ihm. Das individuelle Einzelstück verkommt zur Ware und der soziale Bezug zum Produzenten der Ware entfällt. Kauft man nahezu anonym anstatt beschenkt zu werden, erodieren die sozialen wie historischen Dimensionen und Produkte reduzieren sich auf ihren materiellen Gegenwert in Form eines Preises.
} 
wirtschaftliche und politische Teilaspekte menschlicher Interaktion festgestellt werden, sodass die Begriffe keinesfalls obsolet sind. Entsprechend können Erkenntnisse aus Werken, die menschliches Handeln als rein politisch oder aber rein wirtschaftlich motiviert deuten, zwar nicht in ihrer Absolutheit für diese Studie übernommen werden, wohl aber können ihnen nützliche Erklärungen menschlicher Interaktion entnommen werden. Zur Würdigung des ökonomischen Mehrwerts gemeinschaftlichen Zusammenlebens und sozialen Handelns hat sich in der Literatur ein Diskurs über Sozialkapital herausgebildet, dem für diese Arbeit nützliche Erkenntnisse entnommen werden können.

Über das Konzept von Sozialkapital wird der Nutzen von Beziehungen herausgearbeitet. Kapital ist akkumulierte Arbeit, Sozialkapital entsprechend akkumulierte Beziehungsarbeit. Die Schaffung von Sozialkapital erfordert im Mindesten Zeit, jedoch müssen im Regelfall auch andere Kapitalien wie Finanz- oder Sachkapital (Geld und Waren) investiert werden. Sozialkapital umfasst Ressourcen, die aus der Zugehörigkeit zu einer bestimmten Gruppe gewonnen werden. Es existiert auf der Grundlage materieller oder symbolischer Austauschbeziehungen innerhalb der Gruppe. Für den Einzelnen fungiert es als Multiplikator seiner eigenen Kapitalien, da es auf dem verfügbaren Gesamtkapital der Gruppe beruht. Für jedes Mitglied geltend, hat Sozialkapital so auch einen Multiplikatoreffekt auf das Gesamtkapital der Gruppe. Basierend auf Vertrauen verleiht das Gesamtkapital der Gruppe so jedem einzelnen Mitglied Sicherheit und Kreditwürdigkeit (Bourdieu 1983a: 190-194).

Bourdieu legt dem Sozialkapitalbegriff ein umfassendes Verständnis von Kapital zugrunde. Es existiert sowohl in materieller, als auch ,in verinnerlichter, inkorporierter' Form“ (1983a: 183). Er möchte so der „immanenten Struktur der gesellschaftlichen Welt" (Bourdieu 1983a: 183) gerecht weden und ihr Funktionieren ergründen können. Damit positioniert er sich explizit gegen einen „,wirtschaftswissenschaftliche[n] Kapitalbegriff, [welcher] die Gesamtheit des gesellschaftlichen Austauschverhältnisse auf den bloßen Warenwert [reduziere], der objektiv und subjektiv auf Profitmaximierung ausgerichtet und vom (ökonomischen) Eigennutz geleitet [sei]“ (Bourdieu 1983a: 184). Wirkmächtig - außerhalb Frankreichs - wurde der Sozialkapitalbegriff jedoch erst, als er seitens der Wirtschaftswissenschaften und in der praktischen EZ wieder auf materiell quantifizierbare und damit ökonomisch meßbare Formen heruntergebrochen wurde. So definiert Coleman Sozialkapital als die Beziehungen zwischen Personen, die produktive Aktivitäten generieren (1988: 100f.). Robert Putnam machte unter dem Begriff Sozialkapital Betrachtungen vom gesellschaftlichen Nutzen menschlicher Beziehungen für ein größeres Publikum zugänglich (1993, 1995). Auch wenn er das Konzept ebenfalls breiter anlegte, nahmen internationale Organisationen den Begriff auf, um quantitativ meßbaren wirtschaftlich-materiellen Nutzen sozialer Beziehungen bestimmen zu können. Insbesondere die Weltbankgruppe inkorporiete eine rein ökonomische Auffassung von Sozialkapital und feierte diese schnell als „missing link:“ (Weltbank 1997: 77) zur Armutsbekämpfung. Aufgrund einer 
engen Verknüpfung zu den neu aufkommenden entwicklungspolitischen Ansätzen von guter Regierungsführung und Partizipation erlangte diese Konzeption den Durchbruch (Harriss 2002: 76-79). Die Förderung von Sozialkapital könne sich positiv auf die Übernahme von Eigentümerschaft von Entwicklungsprojekten auswirken und empowerment stärken.

Hier setzt zugleich jedoch fundamentale Kritik am Sozialkapital-Konzept an. Ein zu enger Fokus auf die marktwirtschaftliche Inwertsetzung menschlicher Beziehungen sei unsozial und entpolitisierend, da soziale, kulturelle und politische Aspekte menschlicher Beziehungen ignoriert blieben. John Harriss macht die Weltbank als Speerspitze eines postkolonialen westlich-kapitalistischen Imperialismus aus und deklariert Sozialkapital als trojanisches Pferd zur Eroberung bzw. kapitalistischen Infiltrierung der restlichen Welt (2002). Mit Sozialkapital als Credo wird soziales Handeln einzig an seinem wirtschaftlichen Nutzen bewertet, ein weiterer gesellschaftlicher Mehrwert ausgeblendet und entwicklungspraktisch einzig solches menschliches Handeln gefördert, welches ökonomische Gewinne verspricht. Die Möglichkeit einer Generierung gesellschaftlichen Nutzens abseits marktwirtschaftlicher Prinzipien würde bei der dogmatischen Wertung von sozialem Handeln nach kapitalistischen Maßstäben verneint werden. Aus antieurozentristischer Perspektive ist Sozialkapital demnach ein verfehltes Konzept für außereuropäische Kulturen.

Unabhängig eines Eurozentrismus kann die Förderung menschlicher Beziehungen innerhalb exklusiver Netzwerke - Sozialkapital - auch normativ als negativ betrachtet werden. Denn Personen, die nicht Mitglieder eines sozialen Netzwerkes sind, sind per Definition ausgeschlossen (Harriss 2002: 9f.). Doch auch für Mitglieder kann die Zugehörigkeit Zwängen unterliegen und zu Verpflichtungen und Beschränkungen führen, die als repressiv empfunden werden (Bohle 2005: 70). So ist der Nutzen des Sozialkapitals abhängig davon, wie es eingesetzt wird und auch Bourdieu spricht bereits von den inhärenten Gefahren einer „Zweckentfremdung“" (1983a: 194). Es kann einerseits die kommunale Entwicklung fördern, andererseits ein Mittel zur Exklusion, Unterdrückung oder Behinderung sein (Kay 2005: 165).

Während Bourdieus ursprüngliches Verständnis von Sozialkapital für die Untersuchung gesellschaftlicher Strukturen auf den Fidschi-Inseln einen Mehrwert verspricht, verneint diese Arbeit eine ökonomisierte Betrachtung menschlichen Sozialverhaltens. Aufgrund der Vereinnahmung des Kapitalbegriffs seitens der Ökonomie, verwende ich im empirischen Abschnitt keine Kapitalbegriffe um Missverständnissen vorzubeugen. Ebenso können Konzepte wie politisches Kapital (Baumann \& Sinha 2001) oder Naturkapital (siehe Kap. 4.1.3) nicht anstandslos genutzt werden.

Dennoch können aus den Debatten und kritischen Auseinandersetzungen mit den Kapitalkonzepten für diese Arbeit nützliche Erkenntnisse gewonnen werden. So hilft die Forderung einer Erweiterung der Sozialkapital-Debatte um politische Dimensionen, die Qualität menschlichen Sozialverhaltens ganzheitlicher zu be- 
greifen. Als konstruktive Erkenntnis eignet sich die Betrachtung von Netzwerken zur Erfassung menschlicher Beziehungen. Strukturtheoretische Ansätze unterscheiden zwischen den horizontalen bonding und bridging-Netzwerken, die für Beziehungen in Verwandtschafts- oder zwischen Interessengruppen stehen, sowie vertikalen linking-Netzwerken, mit denen Beziehungen zu Personen und Institutionen mit Macht und Einfluss bewertet werden (Harriss 2002: 10, 85f.). Insbesondere die vertikalen Beziehungen geben Hinweise auf die politischen Mitgestaltungsmöglichkeiten einer Person und sind für dessen Lebensqualität bedeutend, wie ich in Kapitel 4 über Verwirklichungschancen und menschliche Sicherheit genauer erläutern werde. Auch hier sind nicht quantifizierbare Messgrößen wie die Anzahl an Netzwerken oder deren Größe von entscheidender Aussagekraft, sondern die Stärke bzw. Qualität des ihm zugrunde liegenden Vertrauens.

Räumliche Ausdehnungen sozialer Beziehungen hingegen können indirekt hervorragende Indikatoren für das aus ihnen gewonnene Wohlbefinden darstellen. So ist ein vertrautes, freundlich gesinntes nahes Umfeld wichtig für die Identität und Lebensqualität. Zusätzlich jedoch ist anzunehmen, dass eine Streckung enger Beziehungen über gemeinschaftliche und kulturelle Raumdimensionen hinweg Informationen, Inspirationen und Ideen befördert und zur Umsetzung und damit politischer Mitgestaltung anregt. Diese Studie beinhaltet daher eine Untersuchung räumlicher Komponenten sozialer Beziehungen.

In die kritische Diskussion um Sozialkapital ist zudem die für den Kontext dieser Arbeit brauchbare Erkenntnis eingeflossen, dass nicht nur strukturtheoretische, sondern auch handlungstheoretische Ansätze berücksichtigt werden müssen, welche zeigen, wie Sozialkapital erarbeitet und eingesetzt wird (Bohle 2005: 68f.). So können in dieser Studie aussagekräftige Analysen über die Qualität und die Nutzendimensionen von Beziehungen getätigt und fundierte Rückschlüsse auf die Lebensqualität der teilnehmenden Bevölkerung geschlossen werden. ${ }^{23}$

Resümierend bestärkt die Sozialkapital-Debatte die Erkenntnisse der Gabenökonomie insofern, als dass soziale Beziehungen positive wirtschaftliche Implikationen haben können und Vertrauen deren Basis bildet. Neben materiellem Wohlstand birgt ein hohes $\mathrm{Ma} ß$ an Vertrauen in die Mitmenschen vor allem jedoch eine Quelle für Wohlbefinden und stiftet Identität (Groome Wynne 2007: 119-21), zwei Komponenten von Lebensqualität, die nicht rein ökonomisch fassbar sind. Reziprozität schafft hierbei das entscheidende, normative Fundament für die Bildung von Vertrauen in sozialen (Austausch-)Beziehungen. Mauss attestiert reziprokes Handeln zunächst dem pazifischen Raum der Vormoderne. In den empirischen Kapiteln der vorliegenden Studie wird deutlich, dass Reziprozität auch im heutigen kulturellen Wertegerüst und in der Alltagspraxis der Fidschianer einen bedeutenden Stellenwert hat. Bevor ich zu den empirischen Kapiteln komme,

\footnotetext{
${ }^{23}$ In die bisher einzige Studie, die explizit Sozialkapital auf Fidschi untersucht (Sano 2008a; Sano 2008b), ist keine der genannten Kritiken eingeflossen. Für die hiesige Untersuchung bietet sie leider keinen Mehrwert.
} 
möchte ich mich zunächst jedoch konzeptionell den vor Ort entscheidenden globalen Phänomenen der Globalisierung und Transformation widmen. 



\section{Globalisierung und gesellschaftliche Transformation}

We believe that the central challenge we face today is to ensure that globalization becomes a positive force for all the world's people. For while globalization offers great opportunities, at present its benefits are very unevenly shared, while its costs are unevenly distributed. We recognize that developing countries and countries with economies in transition face special difficulties in responding to this central challenge. (UN-Millenniumserklärung 2000)

In den letzten Jahrzehnten sind weltweit gesellschaftliche Diskurse entstanden, die sich kontrovers dem Begriff „Globalisierung“ widmen. In diesem Kapitel wird der vage Begriff von verschiedenen Standorten aus beleuchtet. Die vielseitigen Debatten über die Globalisierung können als Ausdruck dafür verstanden werden, dass sich die Welt in einem raschen Wandel befindet (Dittrich 2004: 1). Für außereuropäische Gesellschaften ist dieser Wandel ähnlich umfassend wie die große gesellschaftliche Transformation Westeuropas und Nordamerikas im Zuge der Industrialisierung. In diesem Kapitel werden die Konzepte von Globalisierung und Transformation pointiert so zusammengestellt, dass sie einem Verständnis gesellschaftlicher Veränderungsprozesse auf den Fidschi-Inseln dienen. Verweise auf die Diskontinuität und Unübersichtlichkeit sowie auf Gefahrenpotentiale eines Wandels helfen dem Verständnis, dass diese Prozesse Unsicherheiten und Ängste in der lokalen Bevölkerung hervorrufen können. Insbesondere der globale Klimawandel stellt in diesem Kontext ein bedrohliches Phänomen dar. Kleine Inselökonomien wie Fidschi verursachen diesen nicht. Gleichwohl gelten kleine Inselstaaten des Südpazifiks als Hauptleidtragende der Auswirkungen globaler Umweltveränderungen.

\subsection{Globalisierung}

Als Startpunkt kann unter Globalisierung ein sich beschleunigender Prozess der zunehmenden internationalen Verflechtung von Ökonomie, Ökologie, Gesellschaft, Politik und Kultur verstanden werden. Räumlich kann Globalisierung als Rekonfiguration von Geographie aufgefasst werden, sodass entfernte Lokalitäten derart verlinkt sind, dass lokale Ereignisse von weit entfernten Geschehnissen beeinflusst werden (Giddens 1999: 84-86). Globalisierung wirkt komplex auf lokale Wirtschafts-, Sozial- und Raumstrukturen. Sie drückt sich dabei in einer Gleichzeitigkeit integrierender und fragmentierender Prozesse aus. Ambivalenzen, widersprüchliche Prozesse und Paradoxien zeichnen sie aus (Dittrich 2004: 9-19). Transkulturalität (siehe Kapitel 2.1.3) wäre in diesem Sinne eine positiv konnotierte kulturelle Auswirkung der Globalisierung. 


\subsubsection{Raumzeitliche Schrumpfung}

Technische Innovationen in den Bereichen der Kommunikation und des Transports der letzten Jahrhunderte ermöglichten die räumliche Ausdehnung menschlicher Handlungsräume. Die Erhöhung von Geschwindigkeiten sorgt relativ gesehen für eine Verkürzung von Distanzen, welche als raumzeitliche Schrumpfungsprozesse wahrgenommen werden können. Mit der nahezu ortsunabhängigen Verfügbarkeit des Internets und der Mobilfunktelekommunikation kann eine räumliche und zeitliche Aufhebung traditioneller Lebensformen einhergehen (Werlen 2014: 59-62).

Wenn die Welt im Zuge der Globalisierung hinsichtlich der Verfügbarkeit von Informationen zu einem ,globalen Dorf" zusammenschrumpft, können sich Moden, Trends und Muster der Lebensführung global verbreiten, sodass Menschen Lebensstile unabhängig ihrer kulturellen Herkunft entfalten. Alternativ zum Bild des raumzeitlichen Schrumpfungsprozesses fasst Giddens die Globalisierung umgekehrt auf als ein „Dehnungsvorgang, und zwar insoweit, als die Verbindungsweisen zwischen verschiedenen gesellschaftlichen Kontexten oder Regionen über die Erdoberfläche als Ganze hinweg vernetzt werden“ (1999: 85).

\subsubsection{Wirtschaftliche Globalisierung}

Nicht nur Wissenschaftler, sondern verschiedene staatliche und zivilgesellschaftliche Akteure wie Regierungen, Medien und NGOs befassen sich mit dem Phänomen der Globalisierung. In den seltensten Fällen werden dabei neutrale Positionen eingenommen, sondern politische Grabenkämpfe ausgefochten. Oftmals werden speziell wirtschaftliche Aspekte der Globalisierung herausgegriffen, um damit Meinungen zu neoliberaler Marktwirtschaft zu rechtfertigen. So versteht der vielzitierte Wirtschaftsgeograph David Harvey unter Globalisierung eine aktuelle Form der „kapitalistischen Produktion und Rekonstruktion von Raum“ (2001: 24). Im Sinne von Marx wird dem Kapitalismus hierbei Krise als Wesensmerkmal attestiert; permanentes wirtschaftliches Wachstum als Ziel und Grundpfeiler des Kapitalismus führen demnach zwangsläufig über die Zeit zur Überakkumulation, verstanden als ein Überschuss an Kapital und Arbeit, welcher marktwirtschaftlich nicht produktiv genutzt werden kann, sodass im Kapitalismus ein Widerspruch bzw. eine Krise innewohnt. Aufgrund des Überschusses kommt es zu massiver Abwertung von Kapital und Arbeit, die sich in Form von Inflation, Bankrotten, Finanz- und Wirtschaftskrisen, Stilllegungen von Produktionsstätten und Massenarbeitslosigkeit äußert. Am Ende steht solch einer Darstellung zufolge die Selbstzerstörung des Kapitalismus fest (Leggewie \& Welzer 2011: 51f.). Zeitlich hinausgezögert wird dieses Ende durch die Ausweitung des Kapitalismus auf zuvor nicht-kapitalistische Bereiche wie der marktwirtschaftlichen Durchdringung staatlicher und öffentlicher Güter und Institutionen. Weiterhin kann sich der Kapitalismus räumlich auf nicht-kapitalistische Territorien ausweiten, worunter eben die heutige Globalisierung gefasst werden kann. In seiner radikalsten Form hält die 
Vernichtung von Menschenleben, Gütern und Produktionsstätten durch Kriege und sonstige soziale Katastrophen, bei anschließendem Wiederaufbau, den Kapitalismus aufrecht (Harvey 2001: 26).

Aus dieser neo-marxistischen, ökonomischen Perspektive heraus kann Globalisierung also als Prozess globalen Ausmaßes kapitalistischer Durchdringung menschlicher Wirtschafts- und letztendlich Lebensweisen verstanden werden. Vorläufer finden sich dann im Imperialismus und dessen politischen Systems der Kolonialisierung, beginnend mit der „Entdeckung“ Amerikas 1492. Denn im Zuge dessen wurden neue Wirtschaftsräume als Produktionsstätten und Absatzmärkte erschlossen und speziell in Krisenzeiten der Mutterländer "nicht verwertbares“ Kapital und „überflüssige“ Arbeitskräfte in diese exportiert (Harvey 2001: 25f.; Peet \& Hartwick 2009: 161-166). Dieser Darstellung zufolge ist diese Frühform der Globalisierung untrennbar mit Sklaverei, der Ausbeutung und Unterdrückung ganzer Völker und Kulturen und rassistischen Rechtfertigungs- und Beschönigungs-Argumentationsmustern verbunden.

Dependenztheorien und postkolonialistische Konzepte spinnen diese Gedanken weiter. Die Geschehnisse haben sich über die Jahrhunderte fest in die Identitäten der beteiligten Gesellschaften verankert und klingen bis heute nach (Said 1978). Denn auch nach der offiziellen Unabhängigkeit der jeweiligen unterdrückten Staaten -, welche zumeist nur aufgrund der Kolonialisierung in nationalstaatsähnliche Korsette gezwängt wurden, - wirken Abhängigkeiten nach (Heidemann 2011: 136-42; Peet \& Hartwick 2009: 166-72, 208-12). Unter der Kolonialpolitik habe sich eine wirtschaftliche Elite im Land etabliert, die von ausbeuterischen Wirtschaftsstrukturen profitiere. Nach der Unabhängigkeit könne diese im Regelfall ihre wirtschaftlichen und politikbeeinflussenden Positionen beibehalten. Einseitige Abhängigkeiten bleiben bestehen. Dieser Argumentationslogik zufolge spaltet die Globalisierung menschliche Gesellschaften, verschärft wirtschaftliche Ungleichheit und verursacht Armut. Diese und ähnliche Argumentationsmuster werden sowohl von weiten Teilen westlicher als auch der nicht-westlicher Gesellschaften geteilt, sodass es eine Vielzahl gesellschaftlicher Akteure gibt, die sich als Gegner der Globalisierung sehen (Mander \& Goldsmith 2002).

Befürworter der Globalisierung hingegen sehen in einem weltumspannenden Transfer von Technologie und Wissen ein ungemeines Potential, dass alle menschlichen Gesellschaften Nutznießer der Globalisierung sein könnten. Gestaltet man diese richtig, so führe der Abbau von Handelsbeschränkungen und Hindernissen der Kommunikation und des Transportes hinsichtlich Raum und Zeit zu einer wirtschaftlichen Annäherung, mildere wirtschaftliche Ungleichheiten ab und bekämpfe Armut (Rogall 2013: 429-433).

Diese Dissertation widmet sich nicht der Frage, welche Position die Realität besser widerspieglt. Stattdessen möchte ich ein Paradoxon aufzeigen: Unabhängig ihrer intrinsischen Wirkungsrichtung stellt die globale Verbreitung von Informationen um Gefahrenpotentiale der Globalisierung ein eigenes Gefahrenpotential dar. Denn die Fidschianer nehmen ihre Gesellschaft als von weltweiten Gescheh- 
nissen beeinflusst wahr. Das Wissen, dass dieses als Globalisierung benennbare Phänomen zu ihrem Nachteil führen könne, kann schnell Ängste und Unsicherheiten schüren. Schlägt sich dies in pauschalen Ressentiments gegen jegliche ,äuBerliche" 24 gesellschaftliche Veränderungen nieder, wird die Gefahr der Globalisierung zur selbsterfüllenden Prophezeiung.

\subsubsection{Sozio-kulturelle Globalisierung und neo-traditionelle Gegenbewegungen}

Die Globalisierung beeinflusst menschliche Gesellschaften nicht nur in wirtschaftlicher Hinsicht, sondern äußert sich ganzheitlicher. Aufgrund der Mobilität des Kapitals verlagern sich Produktionsstandorte beständig. Neue Boom-Regionen können buchstäblich aus dem Nichts entstehen, genauso schnell können etablierte Standorte niedergehen. Auch innerhalb einzelner Regionen, sogar einzelner Städte können gegensätzliche wirtschaftliche Entwicklungen gleichzeitig ablaufen, HighTech-Viertel schießen aus dem Boden, während die direkte Nachbarschaft verslumt (Dittrich 2004: 56f., 71f.). Ohne effektive Formen der Steuerung fragmentieren Gesellschaften zusehends in profitierende und exkludierte Gruppen. Das Auseinanderdriften von Arm und Reich auf engstem Raum kann schnell zu gesellschaftlichen Spannungen führen, die sich gewaltsam entladen.

Aufgrund der durch die technologische Globalisierung geschaffenen einzigartigen Möglichkeiten der Information, Kommunikation und des Transports sehen viele Millionen Menschen weltweit Migration in die globalen Wirtschaftszentren und deren Hinterhöfe als Anpassungsstrategie. Migration steigt trotz zunehmender politischer Hinderung an und kann die jeweiligen Heimatorte entlasten. Damit einhergehend kommt es zur Entterritorialisierung sozialer Kontakte und zu transkulturellem Austausch. Insbesondere neue Kommunikationsplattformen wie soziale Netzwerke im Internet lassen transkulturelle, globale Beziehungsgeflechte entstehen. Hier besteht die Gefahr, dass auf Kosten dieser globalen Nachbarschaften direkte Nachbarschaften verwaisen (Beck 1997: 93).

Weitere ambivalente Trends der Globalisierung sind einerseits die Vereinheitlichung der Kultur, gleichzeitig jedoch eine Re-Lokalisierung. Über das Internet und die Mobilfunktechnologien vereinheitlichen Kommunikations- und Informationsmuster. Globale Unternehmen und deren Markennamen lenken weltweit Modetrends, Ernährungs- und Konsumgewohnheiten und wirken tief in die Freizeitgestaltung ein. Mit den jeweiligen Produkten kann - bewusst und unterbewusst - eine Übernahme der Werte stattfinden, die diese Produkte ausstrahlen.

Gleichzeitig bilden sich lokale Initiativen, die die Globalisierung im Allgemeinen und die Verwestlichung der Werte im Besonderen kritisch hinterfragen und bewusst ablehnen. Konservative Strömungen gewinnen Zulauf, die emotional bis ideologisch argumentierend die Bewahrung gegenwärtiger kultureller Eigenheiten

\footnotetext{
${ }^{24}$ Definitionen, was als extern oder eigen bestimmt wird, unterliegen - gemäß eines transkulturellen Verständnisses von Gesellschaften und einer Akzeptanz des Faktes einer globalen Vernetzung einer Willkür. Diese Willkür mag politischen Zielen geschuldet sein.
} 
und Kulturlandschaften oder die Wiederkehr vergangener Gegebenheiten fordern. Dies kann als Neotraditionalismus gedeutet werden (Abramson 2004). Die Globalisierung führt damit nicht nur zu einer Homogenisierung oder gar Nivellierung kultureller Unterschiede, sondern parallel zum Erstarken des spezifisch Lokalen. Im Extremum bilden sich ethnisch-nationalistische Bewegungen, die Angst vor kulturellem Wandel schüren und gewaltsam gegen Fremde vorgehen. Migration stellt daher einerseits eine Anpassungsstrategie an die Verschiebungen des Kapitals dar. Im neuen Umfeld kann es andererseits jedoch zu beträchtlichen sozialen Spannungen kommen, sofern dort, zumeist mittels politischer Rhetorik geschürt, die Ablehnung einer multikulturellen Gesellschaft verbreitet ist und transkulturelle Vorteile nur bedingt öffentlich wahrgenommen werden.

Lokale Räume werden daher nicht einseitig von der Globalisierung vereinheitlicht. Bei der Raumproduktion spielen lokalspezifische soziokulturelle Eigenheiten eine ebenso bedeutende Rolle, sodass glokale Räume entstehen, in denen globale wie lokale Kräfte in komplexen Zusammenspielen wirken (Robertson 1995: 2835). Als Glokalisierung verstanden kommt es im Zuge der Globalisierung nicht zu einer Homogenisierung von Lebenswelten im Sinne einer globalen Moderne, sondern zu heterogenen Komplexen, in denen transkulturelle wie lokalspezifisch einzigartige Denk- und Lebensweisen ambivalent aufeinander einwirken. Im empirischen Abschnitt wird dieses Geflecht für drei Küstendörfer Fidschis erörtert.

\subsection{Klimawandel als glokales Risiko}

Eng mit der Globalisierung verknüpft treten seit mehreren Jahrzehnten globale Risiken auf, die die Menschheit insgesamt bedrohen. Verortet werden diese gerne in der Atomkraft, dem (Selbstmord-)Terrorismus, den globalen Finanzmärkten und dem Klimawandel (Beck 2007: 37). Gemäß der Risikoformel (UNDP-BCPR 2004: 136; Villagran de León 2006: 311f.) stellt die Größe des Risikos eine Kombination von Schadensausmaß und Eintrittswahrscheinlichkeit dar. Bei den genannten globalen Risiken ist beides nicht einschätzbar. Anstelle einer naturwissenschaftlich-technischen Perspektive hilft die sozialwissenschaftliche Auffassung von Risiko als gesellschaftliches Konstrukt. Ein Risiko existiert demnach nur, wenn die Gefahr wahrgenommen wurde. Die Grenzwerte von Eintrittswahrscheinlichkeiten und Schadenshöhen, die das mögliche Ereignis zum Risiko machen, werden subjektiv gesetzt, das heißt sie unterliegen einer kulturellen Wahrnehmung, Inszenierung und Bewertung (Beck 2007: 36; Dikau \& Pohl 2007: 1034; Wisner et al. 2003: 14). „Risiko bedeutet die Antizipation der Katastrophe“ (Beck 2007: 29). Die Wahrnehmung als Risiko wird damit zur Voraussetzung für Strategien zur Reduzierung von Verwundbarkeit (Krüger \& Macamo 2003: 47).

Menschen in Wirtschaft, Wissenschaft und Politik müssen Entscheidungen treffen, deren Auswirkungen und Reichweiten - räumlich wie zeitlich - in dieser komplexen, dynamischen und global vernetzten Welt nicht einschätzbar sind, sondern in hohem Maße spekulativ. Alle Menschen leben daher in permanenter 
Unsicherheit und sind sich dieser weitestgehend bewusst. Ulrich Beck sieht die Menschheit in einer „Weltrisikogesellschaft“" vereint (2007).

Wie sich die Auswirkungen der globalen Risiken lokal artikulieren ist unklar. Für Fidschi scheinen insbesondere die lokalen Auswirkungen des globalen Risikos Klimawandel von Interesse. Der Klimawandel ist über die klassischen Sinne nur bedingt wahrnehmbar und als komplexes Phänomen der menschlichen Erfahrung nicht unmittelbar zugänglich, sodass die Gefahr unterschätzt wird (Beyerl 2010: 250f.). Dies mag einer effektiven Bekämpfung des Klimawandels entgegenstehen, stärkt jedoch das Sicherheitsgefühl. Im Folgenden möchte ich zunächst Grundlagen des Klimawandels näher vorstellen, bevor dieser näher im Lichte eines glokalen Risikos, also einer lokalspezifisch wahrgenommenen Bedrohung durchleuchtet wird.

\subsubsection{Wissenschaftliche Grundlagen des Klimawandels}

$\mathrm{Ob}$ es einen Klimawandel gibt, und wenn ja, ob dieser menschgemacht ist, lässt sich nach heutigem Wissensstand und heutigen Wissenschaftskriterien nicht absolut einwandfrei klären. Es gibt jedoch messbare Phänomene auf dieser Erde, die sich bisher ausschließlich über einen Klimawandel in Richtung Erwärmung erklären lassen und bisherige Erklärungen des Ausmaßes dieses Klimawandels lassen ausschließlich auf menschlichen Einfluss als wichtigste Erklärungsvariable schlieBen (IPCC 2007a; Rahmstorf \& Schellnhuber 2007: 27-52). Statistische Berechnungsverfahren sind jedoch zwingend Wahrscheinlichkeits-Äußerungen, da bei jeder Modellierung Fehlertoleranzen auftreten. Daher kann seriöse, quantitative, wissenschaftlichen Kriterien folgende Forschung keine absoluten Aussagen treffen; die verschiedenen, teilweise unabhängigen Forschungen liefern allerdings sehr wohl übereinstimmende Darstellungen, die mit hoher Wahrscheinlichkeit korrekt sind.

Klimaveränderungen können für die gesamte Erdzeit attestiert werden, die „natürliche“ Entwicklung würde aktuell jedoch wahrscheinlicher auf eine neue Eiszeit hinauslaufen, denn auf Erwärmung (Rahmstorf \& Schellnhuber 2007: 21f.), sodass menschliches Eingreifen als Erklärung eines Klimawandels in Richtung Erderwärmung angebracht scheint. Ich möchte hier nicht den KlimawandelDiskurs, seine wissenschaftlichen und unwissenschaftlichen Facetten und die dahinterliegenden politischen Motivationen aufschlüsseln, sondern möchte die Grundlagen der Wahrscheinlichkeit einer Erderwärmung mittels dreier Grundaussagen und einer darauf aufbauenden Frage verdeutlichen.

Bei der Verbrennung fossiler Brennstoffe entsteht Koblenstoffdioxid (CO2). Fossile Energieträger enthalten Kohlenstoff, dieser reagiert beim Verbrennen mit dem Sauerstoff in der Luft zu CO2. Optisch anschaulich sei hier der CO2 enthaltende Rauch aus Schornsteinen und Auspuffen erwähnt.

Es existiert ein physikalischer Effekt mit wärmeerzengender Wirkung, der als Treibhauseffekt bezeichnet wird. Es bedarf zweier Komponenten zu einer für diese Studie hinrei- 
chenden Erklärung des mit physikalischen Gesetzen übereinstimmenden Effekts. Stoffe können erstens kurzwellige Lichtstrahlung in langwellige Wärmestrahlung umwandeln, dunkle Stoffe können dies besonders gut, wie beispielsweise an der Hitze schwarzer Autodächer im Hochsommer gut feststellbar ist. Zweitens können Stoffe wie Glas durchlässig für Licht, aber vergleichsweise undurchlässig für Wärme sein. So kann ein Treibhaus- bzw. Glashauseffekt entstehen, indem Lichtstrahlung durch Glas auf einen Gegenstand fällt, der diese in Wärmestrahlung umwandelt, welche wiederum nicht durch das Glas entweichen kann. Besonders anschaulich und nachvollziehbar wird dieser Wärmeeffekt an der Nutzung von Glas-, bzw. Gewächs- bzw. Treibhäusern im Gartenbau.

CO2 und manche andere Gase haben eine Treibhauswirkung. In Laborexperimenten kann die Treibhauswirkung von Gasen, also Lichtstrahlungsdurchlässigkeit und Wärmestrahlungsundurchlässigkeit, nachgewiesen werden. Auch die unterschiedliche Stärke des Treibhauseffekts verschiedener Gase kann angegeben werden, so ist Methan (CH4) etwa 25mal wirksamer als CO2 (IPCC 2007a).

Die entscheidende Frage ist nun, wie sehr sich die anthropogen erzengten Treibhausgase entsprechend der Laborexperimente auf ein so komplexes System wie die Erdatmosphäre auswirken. Diese Frage lässt sich nur mit gewissen Unsicherheiten beantworten. Die nicht-anthropogene Konzentration an Treibhausgasen hat Modellrechnungen zufolge einen Wärmeeffekt auf die globale Mitteltemperatur von $+33^{\circ} \mathrm{C}$, von $18^{\circ} \mathrm{C}$ auf $+15^{\circ} \mathrm{C}$. Der zusätzliche, anthropogene Treibhauseffekt hat höchstwahrscheinlich zu einer zusätzlichen Erwärmung um $0,7^{\circ} \mathrm{C}$ in den letzten Jahrzehnten geführt und wird bis Ende des Jahrhunderts auf $1,1^{\circ} \mathrm{C}$ bis $6,4^{\circ} \mathrm{C}$ ansteigen(IPCC 2007a: 237, 749). Unsicherheiten existieren nicht, ob es eine Erwärmung gibt, sondern wie stark diese ausfällt und in Zukunft ausfallen wird. Schwierig zu modellieren sind insbesondere regionale Verteilungen sowie die genauen Auswirkungen von Rückkopplungseffekten.

Positive (sich selbst verstärkende) Rückkopplungseffekte sind beispielsweise das Austreten von Methan beim Auftauen von Permafrostböden. Weiterhin reflektiert Eis einen Großteil kurzwelliger Lichtstrahlung, schmilzt dieses, treten dunklere Oberflächen mit höherer Wärmeabsorption zutage. Einen negativen (sich selbst abschwächenden) Rückkopplungseffekt könnte eine vermehrte Wolkenbildung durch höhere Verdunstung darstellen, da Wolken zu einem gewissen Grad Sonnenlicht ins Weltall reflektieren.

Es ist weiterhin davon auszugehen, dass mit einer anthropogenen Erderwärmung im Zuge des Klimawandels Veränderungen von atmosphärischen Zirkulationen und Niederschlagsverteilungen einhergehen. So könne sich die Sahelzone ausweiten, der indische Monsun verschieben oder der Golfstrom abschwächen. Weiterhin drohen eine Versauerung der Ozeane und die Erhöhung des Meeresspiegels. Mehreren Modellrechnungen und Observierungen zufolge hebte sich der globale Meeresspiegel im 20. Jahrhundert bereits um 13 bis $30 \mathrm{~cm}$ an (Bittermann et al. 2013: 4). Bis Ende diesen Jahrhunderts wird er selbst bei optimistischen Annahmen von $1,5-2^{\circ} \mathrm{C}$ Erderwärmung um weitere $75-80 \mathrm{~cm}$ angestiegen sein, 
sowie aufgrund der Trägheit des Klima- und Meeressystems unabhängig weiterer Erderwärmung bis ins 23. Jahrhundert weiter steigen (Schaeffer et al 2012: 867f.). Was diese klimatischen Veränderungen für Menschen und ihre Umwelt bedeuten und wie sich die globalen Klimatrends lokal auf menschliche Lebensweisen auswirken, bedarf noch erheblicher Forschung (Gemenne et al 2014: 6).

\subsubsection{Auswirkungen des Klimawandels auf menschliche Gesellschaften}

Sollte die globale Mitteltemperatur um mehr als $2^{\circ} \mathrm{C}$ gegenüber vorindustriellem Niveau steigen, so wird mit nicht mehr kompensierbaren Schäden für die Menschheit gerechnet. Die $2^{\circ} \mathrm{C}$-Leitplanke wird nicht nur auf Grundlage naturwissenschaftlicher, ökologischer und physikalischer Erkenntnisse getroffen, sondern stellt ein politisches Statement dar. Die Festlegung von Kipppunkten in Ökosystemen unterliegt nicht nur biologischen Parametern, sondern ist gleichsam Ausdruck von Wissen und Erfahrung, Ethik und Risikobereitschaft. Kipppunkte können sich daher über die Zeit verändern, insbesondere wenn Anpassungsmaßnahmen greifen, die Toleranzen verschieben (Adger et al. 2009: 6f.). Entsprechend bestehen kulturelle Dimensionen, wie sehr der Klimawandel Gesellschaften und ihre Wertvorstellungen bedroht (Adger et al. 2013: 112).

Die genauen physischen Auswirkungen eines ungebremsten Klimawandels können mit hohen Wahrscheinlichkeiten abgeschätzt werden und lassen drastische Veränderungen vermuten wie einen meterhohen Meeresspiegelanstieg, Zusammenbrüche überregionaler Systeme wie der thermohalinen Zirkulation, des Monsuns oder ENSO (El Nino Southern Oscillation) (Barnett \& Adger 2007: 640). Eine Skizzierung dieser Auswirkungen auf menschliche Lebensweisen erscheint unmöglich, jedoch existieren Schreckensszenarien, deren Realismus nicht einschätzbar ist, sollte die planetare Leitplanke gerissen werden. Doch auch seriöse Quellen beschreiben real existierende Brennpunkte den Klimawandels. Der WBGU geht davon aus, dass sich der Klimawandel auf Länder mit fragiler Staatlichkeit besonders negativ auswirkt, da diese Länder aufgrund mangelnder politischer Stabilität keine hinreichende Anpassung betreiben. Er geht von einer Negativspirale aus, dass die Auswirkungen des Klimawandels die Regionen zusätzlich destabilisieren (2007: 139f; 169-89). Auf den Fidschi-Inseln gab es in den letzten 30 Jahren vier Putschversuche. Ein Anstieg des Meeresspiegels macht sich in kleinen Inselstaaten besonders deutlich merkbar. Zudem ist Fidschi klimabedingten Naturgefahren wie Zyklonen ausgesetzt, die der Klimawandel intensivieren könnte (siehe Kap. 5.3). Im Gegensatz zu Verwundbarkeiten ist über kulturelle, lokalspezifische Anpassungspotentiale bisher wenig erforscht (Adger et al 2013: 114). Eine positive Ausnahme bilden Lata \& Nunn (2011), die zur Erklärung von Wahrnehmungen und Anpassungspotentialen im Fidschianischen Rewa-Delta explizit culturelle Dimensionen wie traditionelle Häuptlingssysteme als Entscheidungsstrukturen und Glaubensvorstellungen hinterfragen. Bei der Frage, was gutes Leben im 
Inselstaat beeinflusst, scheinen das politische System und der Klimawandel im Zusammenspiel wichtige Faktoren zu bilden.

Klimabedingte Untergangsszenarien sind medial global verbreitet. Als globales Risiko verstanden, lässt sich der Klimawandel lokal nicht steuern. Einseitig einer Gefahr ausgeliefert zu sein, erzeugt Unsicherheit. Diese wiederum kann schnell die Ursache von Angst werden, welche sich gewaltvoll gegenüber vermeintlichen Verursachern des Klimawandels entlädt; oder auch als Mob angestachelt gegenüber allem Fremden und Unbekannten. Damit kann die Antizipation der Katastrophe zur Katastrophe führen und die schlimmsten Horrorszenarien werden zu selbsterfüllenden Prophezeiungen. Die globale Verbreitung von Wissen um Gefahren, eine Errungenschaft der Globalisierung, führt zu weiteren, schlimmeren Gefahren - ein weiteres Beispiel für die paradoxen und unkalkulierbaren Auswirkungen der Globalisierung.

Während es reichhaltige Literatur zu den physischen Auswirkungen des Klimawandels gibt, besteht seitens der Sozialwissenschaften noch erheblicher Forschungsbedarf auf die Auswirkungen auf die Menschen. Insbesondere Anpassungspotentiale werden unterschätzt, wenn die Dynamiken und Reflektionfähigkeiten von Kulturen unberücksichtigt bleiben. Der Klimawandel ist oftmals als globales Problem porträtiert, während die Autoren ethnozentrische Weltanschauungen unterliegen. Dissonanzen entstehen bei Handlungsempfehlungen, wenn dabei ortsbasierten Identitäten und lokalen, kulturellen Wertforstellungen nicht entsprochen wird (Adger et al 2013: 112-114).

Direkte Zusammenhänge zwischen gewaltsamen Konflikten und dem Klimawandel sind empirisch nicht belegt und Potentiale friedvoller Anpassung und Kooperation unterschätzt (Gemenne et al. 2014: 2-6; Barnett 2010). Denn neben Schwarzmalerei bieten sich jedoch genauso gut auch hoffnungsvolle Zukunftsentwürfe an. So steckt in jedem Risiko auch eine Chance; im Chinesischen haben beide Wörter den gleichen Wortstamm 机. Dank der Globalisierung rücken Kulturen näher zusammen, ein Bewusstsein über globale Interdependenzen lokaler Handlungen entwickelt sich und entsprechende Verantwortungen können übernommen werden. Dank technologischer Entwicklungen im Kommunikationsbereich können Wissen und Erfahrungen in noch nie dagewesener Stärke ausgetauscht werden und Lernprozesse beschleunigen sich, sodass sich die Kulturen gegenseitig befruchten. Die kommunikativen wie räumlichen Hindernisse zur Kooperation sind gering wie noch nie und das Wissen um die Vielfalt an Handlungsoptionen, effektive Strategien zur Lösung von Problemen zu generieren, ist so ausgeprägt wie noch nie in der Geschichte der Menschheit. Gesellschaftliche Missstände können besser hinterfragt werden, Unrechtszustände überwunden werden und das allgemeine wie individuelle Wohlbefinden ausgebaut werden, da auch autokratische Regime den Informationstausch nicht vollständig unterbinden können. Internationale Plattformen können erstarken und lokale wie globale Herausforderungen können gemeinsam angepackt und gelöst werden. Ethnische wie 
nationale Engstirnigkeit können dank transkultureller Prozesse überwunden werden und ein Klima der Offenheit und Toleranz Fremdem gegenüber erblühen. Globale Sicherheitsrisiken wie der Klimawandel bieten Gelegenheitsfenster zur Stärkung einer internationalen Gemeinschaft.

In der Theorie existiert zusammengefasst also ein hoher Grad an Beliebigkeit, wie sich die Globalisierung und der Klimawandel lokal bis global auswirken können. Diese Studie geht im Sinne der Glokalisation davon aus, dass Menschen nicht einheitlich (re)agieren, sondern lokalspezifisch aufgrund sozio-kultureller Eigenheiten höchst unterschiedlich erfolgreich Anpassungs- und Bewältigungsstrategien entwickeln. Diese Arbeit wird ein Einzelfallbeispiel herausgreifen und für fidschianische Küstendorfbewohner Ursachen wie Wirkungen der Globalisierung und des Klimawandels aufarbeiten, sowie bestehende Versuche der Steuerung skizzieren.

\subsection{Gesellschaftliche Transformation}

Karl Polanyi analysierte mit „The Great Transformation“ (1944/2001) brillant die gesellschaftlichen Veränderungen, die Gesellschaften im 19. Jahrhundert mit dem Einzug der Industrialisierung und damit einhergehenden Marktliberalisierung durchliefen. Ausgehend von Großbritannien weiteten sich die Prozesse auf das europäische Festland, Nordamerika und später Japan aus. Aufgrund der Interdependenz von Wirtschaft und Gesellschaft hatte dieses primär ökonomische Phänomen weitreichende, extreme soziale Auswirkungen und wird daher als "Große“ - weil sämtliche Bereiche gesellschaftlichen Lebens umfassende - Transformation verstanden. Der so verwendete Transformationsbegriff bezieht sich auf den Wandel einer Gesellschaft zur Moderne. Ähnlich stellt Anthony Giddens fest, dass diese Umgestaltung der Gesellschaft vom Traditionalen zum Modernen wesentlich extremer abläuft, als alle vorangegangenen historischen Übergänge der Menschheitsgeschichte. Diese Radikalität bzw. Diskontinuität drückt sich erstens in der schieren Geschwindigkeit des Wandels aus. Zweitens ist die geographische Reichweite dieses Wandels neu. Drittens ist die Tiefe des gesellschaftlichen Wandels mit dem Aufkommen moderner Institutionen und damit neuer sozialer Ordnungen einzigartig (Giddens 1999: 12-15). Um begrifflich die neuen Dimensionen eines gesellschaftlichen Wandels zur Moderne gegenüber sonstigen Phänomenen gesellschaftlicher Umgestaltungsprozesse zu verdeutlichen, spreche ich von einer Transformation.

Konzeptionell kann der tiefgreifende Wandel durch Koevolution erklärt werden, dass sich Wirtschaft, Wissenschaft, Kultur und technischer Fortschritt wechselseitig und dynamisch so beeinflussen, dass sie in Kombination einen gesellschaftlichen Umbruch herbeiführen, ohne dass dieser gezielt gesteuert wird. Dieser Übergang hat dabei nicht zwingend ein eindeutiges Zentrum, kann sich „schleichend“ über Jahrzehnte hinweg manifestieren und der Wandel kann diskontinuierlich von Rückschlägen sowie zeitlichen Versetzungen und wechselnden 
Tempi geprägt sein; nichtsdestotrotz entsteht eine Häufigkeitsverdichtung von miteinander konform gehenden Veränderungen, sodass der Radikalität und Stoßrichtung des Umbruchs kein Abbruch getan wird (Grin et al. 2010, WBGU 2011: 89-95).

Ein Grundpfeiler der Großen Transformation im Westen war technischer Fortschritt. Mit der Erfindung der Dampfmaschine und der Erschließung fossiler Energien revolutionierten Dampfschiff und Eisenbahn den Transport. Zuvor war man von Wind, eigener Muskelkraft und Nutztieren abhängig. Neben Schienennetzen wurden Kabel über Fernstrecken verlegt, die Langstreckenkommunikation über Telegramme und später das Telefon ermöglichten. „Durch die technische Möglichkeit der Gleichstellung von Uhren entstand das Zeitalter der Fahrpläne, Zeittaktungen und der Beschleunigung von Arbeitsabläufen" (WBGU 2011: 95). Die Folge waren neue Zeit- und Raumverständnisse. Die neuen Technologien zu Kommunikation und Transport intensivierten Technologietransfers und internationalen Handel, mit dem ein kultureller Austausch einherging.

Parallel vollzog die Gesellschaft einen Wandel von einer ländlichen Agrargesellschaft zu einer urbanen Industriegesellschaft, mit neuen sozialen Strukturen, Kunstformen und Lebensstilen. Die Handlungsmöglichkeiten erweiterten sich und die Gesellschaften wurden ausdifferenzierter. Die Aufklärung schritt immer weiter voran und kulturelle Leitbilder von Vernunft und Freiheit gewannen Zuwachs. Insbesondere für diesen kulturellen Wertewandel brauchte es Vordenker wie Immanuel Kant, Karl Marx, Jean-Jacques Rousseau oder Adam Smith die auch wenn sie noch so unterschiedlich argumentieren - Narrative für gesellschaftlichen Wandel entwarfen und Werte schufen. Ihre Ideen klingen bis heute nach und geben weiten Teilen der Gesellschaft Orientierung. Allgemein konnten politische Strukturen, die dem Adel von Gott gegebene Privilegien, Macht und Gewalt zuschrieben, kritischer hinterfragt werden. Ein Wandel von einer Stände- zu einer Klassengesellschaft sowie von Monarchien zu demokratieähnlichen Herrschaftsformen vollzog sich. Umgekehrt bildeten sich neue Unfreiheiten. „Der industrielle Arbeitstag mit seinem abstrakten Zeitregime standardisiert die Handlungsmöglichkeiten und Handlungen des Einzelnen viel stärker, als das unter agrarischen Produktionsverhältnissen sinnvoll und notwendig gewesen war" (Leggewie \& Welzer 2011: 179).

Als ein elementares Wesensmerkmal und Motor der Transformation attestiert Polanyi das Vordringen kapitalistischer Denk- und Wirtschaftsweisen in die Gesellschaft. Wissenschaft und Wirtschaft vernetzten sich, sodass eine Bereitschaft der Vermögenden entstand, Kapital in Technologien zu investieren. Medizinischer Fortschritt führte zu Bevölkerungswachstum, der Migrationsprozesse in die Städte förderte, wo Fabriken Arbeit nachfragten. Nicht nur internationaler Handel sondern vor allem auch Binnenhandel intensivierten sich, da Fabrikarbeiter keine Selbstversorger waren und aufkommende Mittelschichten die Nachfrage nach Gütern des gehobenen Bedarfs steigerten. Das Narrativ vom Wohlstand festigte sich. 
Nach post-marxistischem, antikapitalistischem Verständnis fußen die kapitalistischen Produktionsweisen und unternehmerischen Gewinne dabei auf der Ausbeutung der Arbeiterklasse und dem Abbau fossiler Energieträger. Nach Karl Polanyis Lesart wurden Privatisierung, Deregulierung und Marktliberalisierung idealisiert und die menschliche Gesellschaft zunehmend einem selbstregulierenden Markt untergeordnet. Dies unterwanderte zwischenmenschliche Austauschbeziehungen und entmenschlichte die Gesellschaft. ${ }^{25}$ Neben Ausbeutung als einer Form von Ungerechtigkeit unterstellt Max Weber dem Kapitalismus dabei zusätzlich eine Unterminierung weiterer Leitbilder jener Gesellschaften:

\begin{abstract}
Es ist höchst lächerlich, dem heutigen Hochkapitalismus, wie er jetzt nach Rußland importiert wird und in Amerika besteht, - dieser »Unvermeidlichkeit «nserer wirtschaftlichen Entwicklung, - Wablverwandtschaft mit »Demokratie« oder gar mit »Freibeit« (in irgend einem Wortsinn) zuzuschreiben, während doch die Frage nur lauten kann: wie sind, unter seiner Herrschaft, alle diese Dinge überbaupt auf die Daver smöglich«? Sie sind es tatsäcblich nur da, wo dauernd der entschlossene Wille einer Nation, sich nicht wie eine Schafherde regieren zu lassen, dabinter steht. (Weber 1917: 63f.)
\end{abstract}

\title{
3.3.1 Globalisierung als Motor heutiger Transformationsprozesse
}

In außereuropäischen, ehemals vormodernen, von Gabenaustausch geprägten Gesellschaftssystemen wie auf den Fidschi-Inseln haben im Zuge der Globalisierung bzw. seit der Kolonialisierung gesellschaftliche Umbruchprozesse eingesetzt. Diese weisen Analogien zur Großen Transformation auf, sodass der Transformationsbegriff beibehalten wird.

Wieder modifizieren sich Wirtschaft, Wissenschaft, Kultur und technischer Fortschritt koevolutionär und die Veränderungen verdichten sich dynamisch zu einem gerichteten, wenn auch nur bedingt gesteuerten, umfassenden und komplexen Wandel. Weltweit erhält die liberale Marktwirtschaft Einzug in außereuropäische Kulturen und das Narrativ vom Wohlstand verbreitet sich. Fortschritt und Entwicklung sind global auserkorene Ideale, die mit der Wirtschaft verzweigte Wissenseliten produzieren; Bildung wird institutionalisiert. Globale soziale Beziehungsgeflechte lassen multi- und transkulturelle Gesellschaften entstehen. Manche Werte finden global Zustimmung. Exemplarisch zeigen dies die Universelle Menschenrechtserklärung (UN 1948), die aus der UN-Millenniumserklärung hervorge-

\footnotetext{
${ }^{25}$ Für ein Verständnis von Transformationsprozessen auf Fidschi nicht entscheidend, aber zur kritischen Hinterfragung dieser Darstellung möchte ich erwähnen, dass Polanyi aus seinem Zeitgeist heraus argumentiert. Er sieht den Wandel zur liberalen Marktwirtschaft besonders kritisch und benutzt Begriffe wie „debumanization“ (Polanyi 1944/2001: 102, 300) oder „annibilating the buman and natural substance of society" (Polanyi 1944/2001: 3). Denn er möchte erklären, dass aus der Großen Transformation eine zweite hervorging: der Faschismus. Neben Polanyi sehen auch Soziologen und Politologen gerade im Vormarsch der (Post-)Moderne und damit verbundenen Individualisierung, Entwurzelung und Kontaktlosigkeit die Möglichkeiten der Entstehung eines Totalitarismus gegeben (Arendt 1955: 507f.; Behrends 2010: 40-42; Giddens 1999: 17; Luhmann 2010: 257f.).
} 
gangenen Millenniumsentwicklungsziele (UN 2000) oder die Welterklärung zur Demokratie der Interparlamentarischen Union (IPU 1997: 10f., Annex I, Annex VII), welche stets von allen Mitgliedern ohne Gegenstimme akzeptiert wurden. Ermöglicht werden diese Entwicklungen durch neue Transport- und vor allem Kommunikationstechnologien, die Raum und Zeit neu konfigurieren. Immer noch fußt die Entwicklung auf der Nutzung fossiler Rohstoffe, sowie nach postmarxistischem Verständnis auf postkoloniale Ausbeutung ehemaliger, dennoch abhängiger Kolonien (Peet \& Hartwick 2009: 208-214).

\subsubsection{Transformation als Unsicherheit}

Während menschliche Gesellschaften permanent Veränderungen unterliegen, zielt der Transformationsbegriff auf einen beschleunigten, sprunghaften, umfassenden und radikalen Umbruch ab. Neue Freiheiten und Handlungsmöglichkeiten können dazu genutzt werden, sich sozialer Kontrolle zu entziehen und kulturellen Werten zu entsagen - beispielsweise in der Anonymität einer Großstadt. Durch die rapide Veränderung einer Kultur, ihrer Werte sowie ihres Erfahrungs- und Handlungsraumes, der die eigenen Mitglieder nicht Schritthalten können, schwindet der Erwartungshorizont und Vertrauens- und Identifikationsbasen werden unterminiert; das Eigene wird als fremd empfunden. Unsicherheit verbreitet sich. Die gehäuft auftretenden Veränderungen, egal ob singulär betrachtet vermeintlich positiver oder negativer Wirkung, können Gesellschaften destabilisieren.

Unter dem Schwinden von Erwartungen an und Vertrauen in seine Mitmenschen leidet sozialer Zusammenhalt. Wenn soziale Gefüge zusammenbrechen, indem soziale Beziehungen wegbrechen oder aber sich derart modifizieren, dass Erwartungen an sie nicht erfüllt werden, brechen materielle wie psychologische Sicherheiten weg. Die Gabe als Kernelement von sozialem Ausgleich, kulturellen Gerechtigkeitsverständnissen und damit einhergehender sozialer Sicherung wird nicht mehr praktiziert, wenn individuelle Hoffnungen des Gebers auf Reziprozität - sei es in Form materieller Gegengabe, Status, Macht oder Anerkennung - nicht mehr erwartet werden können.

Gerade in Zeiten der Transformation brechen tradierte Eliten weg. Neuen Eliten fehlt hingegen noch die breite Zustimmung im und Legitimation durch das Volk. Sie agieren losgelöster vom gesamtgesellschaftlichen Wertekodex und nutzen ihre Machtpositionen daher ggfs. weniger als vorherige Eliten zur Förderung von als verwundbar anerkannten und als förderungswürdig erachteten Gruppen. Stattdessen betreiben sie zur Aufrechterhaltung ihrer Macht einen neuen Klientelismus im Vergleich zu alten Eliten, sodass bestimmte soziale Gruppen und Minderheiten profitieren, andere hingegen leiden. Lokal wie national entmachten gesellschaftliche Regulierungen zur Verhinderung extremer Formen von Ungerechtigkeit (Tröger 2003: 24), wenn traditionelle Sicherungssysteme nicht mehr greifen. Gerade bei beschleunigten Veränderungen, wie beispielsweise die von IMF und Weltbank geforderten ökonomischen Reformen in Strukturanpassungsprogram- 
men, sprengen aufgrund der Interdependenz des Sozialen und Ökonomischen soziale Kohäsionen und führen gar zu Gewalt (Stiglitz 2001: x-xiii).

Jedoch sind auch positive gesamtgesellschaftliche Veränderungen im Zuge einer Transformation denkbar. So können bestehende Unrechtszustände überwunden werden und die Transformation weckt Hoffnungen auf ein humaneres Gesellschaftssystem (Giddens 1999: 16). Der WBGU sieht in der Transformation eine Chance, diese aktiv zur Bekämpfung globaler Umweltprobleme zu steuern und Gesellschaften entsprechend des Ideals der Nachhaltigkeit zu gestalten (2011: 1). Das empirische Kernanliegen dieser Arbeit ist eine Analyse, inwieweit das Postulat der Unsicherheit im Zeichen von Transformation auf die sozio-kulturellen Spezifika Fidschis zutrifft, und dabei Praktiken zu erkennen, die soziale Sicherheit trotz gesellschaftlicher Transformation bewahren und sogar ausbauen. 


\section{Lebensqualität - Soziale Sicherung durch Verwirklichungschancen}

Diese Arbeit möchte einen Beitrag zur Steigerung von Wohlbefinden und Lebensqualität fidschianischer Küstendorfbewohner leisten. Nach Möglichkeit soll dies nicht auf die primäre Zielgruppe beschränkt bleiben, sondern auch das Leben weiterer menschlicher Gesellschaften bereichern, keinesfalls jedoch auf Kosten anderer passieren. Als Ausgangspunkt zur Überlegung, was Wohlbefinden und Lebensqualität theoretisch ausmacht, starte ich mit Konzepten über Entwicklung, sozialer Verwundbarkeit und menschlicher Sicherheit. Daran anschließend wird ein integrativer, wertebasierender Ansatz entwickelt, um die Lebensrealitäten auf Fidschi bestmöglich erfassen zu können. Dies bildet die theoretische Grundlage für das kulturelle Wertefundament der Menschen, die im Zentrum dieser Studie stehen. Im empirischen Teil der Studie wird dieses mit konkreteren Inhalten gefüllt.

\subsection{Eurozentristische Vorstellungen von Armut und Entwicklung}

Diese Arbeit lässt sich der geographischen Entwicklungsforschung zuordnen, welche sich stets kritisch mit ihrem eigenen Verständnis von Entwicklung auseinandergesetzt hat (KORF \& ROTHFUSS 2016: 164-166). Zunächst möchte auch ich den Entwicklungsbegriff daher kurz skizzieren. Am Ende des Zweiten Weltkriegs, mit dem der Niedergang der Kolonialreiche und eine Welle an Unabhängigkeitsbestrebungen einsetzten, gewann international die entwicklungspolitische Zusammenarbeit an Interesse. Die beiden Bretton-Woods-Organisationen Internationaler Währungsfonds (IWF) und Weltbank wurden 1944 gegründet. Mit dem Ziel wirtschaftlicher Entwicklung unterentwickelter Länder wurde zunächst der Wiederaufbau nach dem Krieg angekurbelt, später standen jene afrikanischen, lateinamerikanischen und asiatischen Länder im Fokus, die sich im Zuge des Kalten Krieges zum Westen bekannten.

\subsubsection{Entwicklungstheorien}

Theoretisch untermauert wurde der Entwicklungsbegriff über die Modernisierungs- und Dependenztheorien. Die Modernisierungstheorien bescheinigen weniger entwickelten Ländern eine wirtschaftliche und kulturelle Rückständigkeit, die mittels nachholender Entwicklung im Sinne eines Nachahmens westlicher Wirtschaft und Kultur beseitigt werden könne. Finanzarmut bestimmt dabei den Gradmesser für Unterentwicklung; das Bruttoinlandsprodukt ist die Bewertungsgrundlage (Engelkamp \& Sell 2013: 195-211; Rogall 2013: 210-221). Als Entwicklungsideal wird die westliche Massenkonsumgesellschaft gepriesen, Entwicklung selbst sei ein gradliniger Prozess. Internationaler Handel sei der Schlüssel zum 
Erfolg. Lässt man das ethnozentristische, diskriminierende Moment kultureller Rückständigkeit außen vor, so ist die Theorie von einem großen Optimismus geprägt, dass sich alle Menschen, Länder und Regionen der Welt positiv entwickeln und das Narrativ vom Wohlstand - diesen westlich-eurozentrischen Grundwert - für sich alsbald in Anspruch nehmen können.

Die Dependenztheoretiker hingegen sahen im Vormarsch kapitalistischer Wirtschaftsweisen, insbesondere des internationalen Handels, ein globales System der Ausbeutung von Entwicklungsländern. Sie bescheinigten den neuen, formal unabhängigen Staaten eine einseitige wirtschaftliche Ausrichtung auf das ehemalige Mutterland. Aufgrund der ungleichen Handelsvoraussetzungen, einem reichen Mutterland und einer armen ehemaligen Kolonie, dominiert das Mutterland die Wirtschaftsbeziehungen und stürzt den Handelspartner über die ungleichen Machtstrukturen in neue Abhängigkeiten. Aufgrund der einseitigen Exportorientierung bei Wirtschaftsbahnen, die Güter wie Gewinne aus dem Land extrahieren, verarmt das Entwicklungsland. Der Westen entwickle sich aktiv auf Kosten der ehemaligen Kolonien, forciere Unterentwicklung. Im Vergleich zum Denkmodell des Wohlstands für alle der Modernisierungstheoretiker kennzeichnet die Dependenztheorien eine pessimistische marktwirtschaftliche Weltanschauung. Eine möglichst gänzliche Abkopplung vom Westen sei die einzige Möglichkeit, ein bisschen Wohlstand behalten zu können.

Beide Großen Theorien gelten als gescheitert. Experten aus Wissenschaft und Entwicklungspraxis wenden sich seit den 1980er Jahren zunehmend von solchen Pauschallösungen für die spezifischen Probleme einzelner Länder und Regionen bzw. ihrer Bewohner ab (Thiel 2001: 9-11) und wollen stattdessen individuelle Strategien entwickeln. Dementsprechend wird zuallererst das dieser Arbeit zugrundeliegende Verständnis von Entwicklung erläutert.

Entwicklung bezeichnet sowohl einen Prozess als auch einen Zustand. Als Prozess verstanden ist Entwicklung in erster Linie nicht geradlinig und einheitlich, sondern vor allem zirkulär, sprunghaft und inkonstant. Entwicklung ist nicht unilinear, sondern von mehrdimensionalen, teils gegensätzlichen Dynamiken und zumindest scheinbaren - Rückschritten gekennzeichnet. Wird normativ in Retrospektive von Entwicklung besprochen, so ist das Gesamtbild positiv. Als Zustand verstanden ist Entwicklung daher ein Idealzustand. Zustand und Prozess sind dann gerne verstanden als ,,menschenwürdige und nachhaltige Entwicklung“ (Uwe Holtz zitiert nach Deutscher \& Ihne 2010: 13).

Vor allem ist Entwicklung kein ausschließlich wirtschaftliches Phänomen, sondern zieht alle Bereiche menschlichen Lebens in Betracht. Idealerweise soll jede Gesellschaft den Zustand und den Weg dorthin selbstbestimmt definieren. Erschwerend kann dabei jedoch die Gesellschaft, die die jeweilige Definitionsmacht innehat, nicht eindeutig bestimmt, geschweige denn als homogene Masse verstanden werden (siehe Kap. 2). Entwicklung ist auch innerhalb ihrer Deuter ein Streitthema, deren Ausarbeitung politischen Aushandlungsprozessen unterliegt bzw. unterliegen soll. 


\subsubsection{Nachbaltige Entwicklung}

Der Begriff der Menschenwürde wird im Verlauf dieses Kapitels noch genauer konzeptionell untermauert und im empirischen Teil der Arbeit mit Inhalten gefüllt. Auf das Konzept von Nachhaltigkeit möchte ich an dieser Stelle bereits eingehen. Gängige Definitionen verstehen unter nachhaltiger Entwicklung eine Entwicklung, die die Bedürfnisse der heutigen Generation befriedigt, ohne die Möglichkeiten der Bedürfnisbefriedigung zukünftiger Generationen zu beeinträchtigen (Brundtland 1987: 37). Auf der UNO-Konferenz 1992 in Rio de Janeiro wird Nachhaltigkeit zum Leitbegriff erhoben und seither in vier Dimensionen gedacht:

- Wirtschaftliche Nachhaltigkeit: ein Einkommen und Arbeitsplätze schaffendes Wirtschaftswachstum;

- Soziale Nachhaltigkeit: gerechtes und sozial verträgliches intra- wie intergenerationelles Wirtschaften;

- Politische Nachhaltigkeit: eine der Partizipation, der Demokratie und den Menschenrechten verpflichtete politische Ordnung; und

- Ökologische Nachhaltigkeit: Umweltverträglichkeit. Vollständige ökologische Nachhaltigkeit meint eine Ressourcennutzung, bei der nur so viele Rohstoffe verbraucht werden, wie nachwachsen, sodass eine Nutzung endlicher Ressourcen dem Konzept entgegensteht.

Das Konzept, welches insbesondere die ökologischen Dimensionen von Entwicklung hervorhebt, gilt als holistisches Konzept, welches auf nachfolgenden Weltkonferenzen viel Bestätigung und Anerkennung bekam (Holtz 2011: 115-117). Es gibt jedoch berechtigte Kritik, dieses westliche Konzept würde anderen Kulturen aufgedrängt, infiltriert diese und beraubt Länder ihrer Souveränität. Nachhaltigkeit ist in ihrer Umsetzung ein technokratisches, marktwirtschaftliches Konzept die Umwelt zu managen, weshalb vorweg die Weltanschauung steht, Gesellschaft und Natur seien getrennt zu betrachten (Escobar 1996: 327-332). Genauso hat sich abermals die künstliche Spaltung des gesellschaftlichen in sozial, wirtschaftlich und politisch etabliert. Diese Dichotomien unterwandern das Selbstverständnis von Menschen nicht-westlicher Kulturen. Der globale Diskurs trägt demnach eine eurozentristische Note.

\subsubsection{Mensch-Natur Dichotomie}

Im Zuge der Aufklärung und seither über Generationen und mit Abstrichen über Kontinente hinweg hat sich der dichotome Gedanke von Mensch und Natur verankert. Der Mensch stünde demnach getrennt von der Natur bzw. normativ gesehen über ihr. Sie sei ihm feindlich gesinnt, und man müsse gegen sie arbeiten. Im hobbesschen Sinne sei menschliches Leben im Naturzustand „einsam, armselig, ekelhaft, tierisch und kurz" (Hobbes 1651/2004: 111) und zivilisatorischer Fortschritt die Loslösung des Menschen von der Natur. Dieses Ideal schwingt in westlichen Entwicklungsvorstellungen zumeist implizit mit. So bändige der Mensch 
über Umwelt-Management die Natur und könne sie als Dienstleister wirtschaftlich nutzen. Diese Arbeit jedoch schätzt Menschen als plurale Wesen und folgt einem anderen Narrativ.

Dass „die Natur“ nicht zu bändigen ist, zeigen die jüngsten katastrophalen $\mathrm{Na}$ turereignisse wie der Zyklon Pam im März 2015 über Vanuatu, Super-Taifun Haiyan Ende 2013 auf den Philippinen, das Erdbeben und der anschließende Tsunami im März 2011 vor Fukushima, Japan, das Erdbeben in Haiti Anfang 2010, Hurrikan Katrina in New Orleans Ende August 2005, oder der Tsunami Weihnachten $2004 \mathrm{im}$ Indischen Ozean, zumal auch die technisch hochentwickeltsten Nationen nicht gefeit sind. Das Herausgreifen solch seltener Extremereignisse verstärkt allerdings den Fehleindruck, die Natur wäre uns feindlich gesinnt. Entsprechende Literatur zu Verwundbarkeit und Naturgefahren fördert oftmals diese verzerrte Weltanschauung.

Anerkannte Betrachtungsweisen legen umgekehrt nahe, Böden vulkanischen Ursprungs seien besonders fruchtbar und die regelmäßige Überschwemmung des Nils hätte wesentlich zur Herausbildung einer frühen Hochkultur beigetragen. Ohne das wärmende Licht der Sonne und die Reinigung von Luft und Wasser seitens der biologischen und abiologischen Natur wäre menschliches Leben nicht denkbar. Außereuropäische Gesellschaften können die Ansicht einer Trennung von Mensch und Natur als losgelöst voneinander nicht zwingend nachvollziehen. Auch eine Stigmatisierung der Natur und ein Arbeiten gegen sie mag normativ auf Unverständnis stoßen. Eine Unterordnung der Natur, die der ManagementGedanke enthält, kann auf Ablehnung stoßen. So kann es ein gesellschaftliches Ziel sein, im Einklang mit ihr zu leben, damit Naturereignisse keine (sozialen) Katastrophen darstellen. Auch ist „Mutter“ Natur kein Dienstleister, da sie gibt, ohne Gegenleistungen zu verlangen. Normativ sollte nicht Ausbeutung und Gegenarbeiten, sondern Dankbarkeit, ggfs. Reziprozität unser Handeln bestimmen. Insbesondere unter dem Stichwort „Gaia“ werden zunehmend auch in der westlichen Wissenschaftswelt Konzepte diskutiert, die die Natur bzw. Leben ganzheitlicher in der Verflechtung mit der biologischen wie nicht-biologischen Umwelt betrachten wollen (Herrmann-Pillath 2011; Kleidon 2010; Lovelock 1991: 10-13; Sutton 2004: 77f.).

Ein primär wirtschaftliches Verständnis von Natur als zu managende Umwelt, die durch Arbeit in Naturkapital umzuwandeln sei, greift zu kurz um Herausforderungen zu meistern. Gerade die Ökonomisierung des Nachhaltigkeitsgedankens führt zu Formulierungen wie „Ein wichtiges Ziel nachhaltiger Entwicklung ist der Erhalt der Leistungsfähigkeit der Ökosysteme“" (BMZ 2013: 102, eigene Hervorhebung). Dies reduziert den Wert der Natur auf ihr Potential, wirtschaftlich genutzt werden zu können und kann für viele Kulturen eine Zumutung darstellen. Der Einbezug der Natur in marktwirtschaftliche Systeme kann Werte verletzen und Glaubensvorstellungen diskriminieren. Versuche einer Standardisierung und Verpreislichung können Phänomenen ihre Einzigartigkeit, Heiligkeit und Wertigkeit rauben. Da der globale Nachhaltigkeits-Diskurs vom Westen und dessen 
Weltanschauungen beherrscht wird, müssen nach Arturo Escobar nicht-westliche soziale Bewegungen und Gemeinschaften alternative Strategien artikulieren, um nicht von konventionellen, kapitalistischen Entwicklungspfaden überrannt zu werden (1996: 339). Der Einhalt westlicher Vorstellungen von Wirtschaft und Entwicklung und daraus abgeleitete Maßnahmen können demnach das Wohlbefinden schmählern.

Unterscheidungen in dieser Dissertationsschrift zwischen dem „Westen“ auf der einen Seite und dem „globalen Süden“ bzw. den „Entwicklungsländern“ bzw. schlicht dem „Rest der Welt“ auf der anderen Seite sollen kein verkürztes, dichotomes Weltbild zeichnen (Bhabha 1994). Wir haben es mit hybriden, vielfältigen Gebilden zu tun, innerhalb derer und - für die hiesige Argumentation wichtiger zwischen denen kulturelle Unterschiede bestehen, trotz mannigfaltiger, gegenseitiger Befruchtung und Überschneidung. Im kulturellen Rahmen Fidschis - ohne diesem Rahmen klare physische oder immaterielle Grenzen ziehen zu wollen findet das primär westliche Konzept nachhaltiger Entwicklung graduelle Entsprechungen. Dafür muss ich keinesfalls in neokolonialer Weise Leitbilder zur Gestaltung der Umwelt von Ländern aufoktruieren, die die heutigen ökologischen Problemlagen durch teils 200jährige, ressourcenvernichtende Industrialisierung erst geschaffen haben (Gebhardt et al. 2011b: 23).

In Fidschi existieren Werte und Normen, wie Nächstenliebe, der schonende, respektvolle, sparsame und bedachte Umgang mit Gottes Schöpfung, oder die Übernahme von Verantwortung für nachfolgende Generationen (Vielzahl von Einzel- und Gruppengesprächen in allen Untersuchungsdörfern), die Koinzidenzen mit dem Konzept von Nachhaltigkeit aufweisen. Zudem sind ihnen als hybride Kultur im Transformationsprozess Ansätze zum Umwelt- und RessourcenManagement nicht unvertraut. Im weiteren Verlauf dieser Arbeit wird der Begriff der Nachhaltigkeit in Ermangelung sprachlicher Alternativen daher übernommen (Genauere Erläuterungen zu Werten in der fidschianischen Kultur und einer nachhaltigen Alternativ-Strategie ökologischen Handelns finden sich in Kapitel 7.4.).

\subsubsection{Kultur, Entwicklung und die Entzauberung der Welt}

Aus der bisherigen Argumentation wird deutlich, dass Entwicklung in einem engen Zusammenhang mit Kultur steht. Entwicklung kann nicht als ein primär ökonomisches Ziel verstanden werden, Finanzarmut zu überwinden, sondern ist ein ganzheitliches, normativ besetztes gesellschaftliches Phänomen. So besteht der Sinn von Entwicklung keineswegs darin, Menschen reicher zu machen, sondern menschliches Leben zu bereichern. Und hierfür muss man auf Werte schauen, welche wiederum kulturell unterlegt sind.

Ob Literatur, Musik, Kunst oder sonstige kulturelle Ausdrucksformen; diese schätzen wir und sie bereichern unser Leben. Finanzieller Wohlstand kann lediglich als einer von vielen Indikatoren herhalten, Möglichkeiten zu schaffen, Le- 
B: Theoretisch-konzeptioneller Rahmen

bensqualität zu verwirklichen. Doch selbst für den Erfolg von Entwicklung als ökonomische Bestimmungsgröße stellen kulturelle Faktoren eine entscheidende Grundvoraussetzung dar. Ein Umfeld gegenseitigen Vertrauens und hoher Verlässlichkeit, sowie die Abwesenheit von Korruption und organisierter Kriminalität stellen sozio-kulturelle Grundpfeiler wirtschaftlicher Entwicklung dar. Ferner führt eine Kultur der Angst und Unterdrückung zu Skepsis und Apathie, die Entwicklung in wirtschaftlicher wie ganzheitlicher Hinsicht lähmen. Umgekehrt ist eine Kultur der öffentlichen Diskussion, Freiheit und politischer Partizipation entscheidend für die Chancen auf Selbstverwirklichung, Lebensqualität bzw. Entwicklung (Ostrom 2000: 140-145; Sen 2004a: 39-43; Sen 2004b).

Max Weber sieht den (wirtschaftlichen) Rationalismus als ein abendländliches kulturelles Konstrukt an. Rationalität und mathematische Logik im Zuge der Aufklärung sei demnach lediglich die Ersetzung einer Glaubensvorstellung durch eine andere. Diese führe nicht zwingend zu einer Verbesserung der Lebensqualität, sondern beraube der Welt ihren Zauber:

\begin{abstract}
Die zunehmende Intellektualisierung und Rationalisierung bedeutet also nicht eine zunehmende allgemeine Kenntnis der Lebensbedingungen, unter denen man steht. Sondern sie bedeutet etwas anderes: das Wissen davon oder den Glauben daran: daß man, wenn man nur wollte, es jederzeit erfahren könnte, daß es also prinzipiell keine geheimnisvollen unberechenbaren Mächte gebe, die da hineinspielen, daß man vielmehr alle Dinge - im Prinzip - durch Berechnen beherrschen könne. Das aber bedeutet: die Entzauberung der Welt. Nicht mehr, wie der Wilde, für den es solche Mächte gab, muss man zu magischen Mitteln greifen, um die Geister zu beherrschen oder zu erbitten. Sondern technische Mittel und Berechnung leisten das. (Weber 1919: 488)
\end{abstract}

\title{
4.2 Soziale Verwundbarkeit
}

Entwicklung und nachhaltige Entwicklung sind Konzepte, die sehr ökonomisch angedacht sind. Betrachtet man die Entwicklungspolitik in Entwicklungsdekaden, so stehen die 1970er Jahre für einen „Krieg gegen die Armut“, der vom damaligen Weltbankpräsidenten Robert McNamara eingeleitet wurde. Über Wirtschaftswachstum soll Armut bekämpft und somit Entwicklung geschaffen werden. Die folgende Dekade gilt als „,verlorenes Jahrzehnt“ (Nuscheler 2005: 79-81), da Wirkungsevaluierungen der Entwicklungszusammenarbeit keine Verbesserungen auf der Makro-Ebene feststellen konnten. Insbesondere die Entwicklungspolitik der 1980er Jahre war von der neoliberalen Wirtschaftspolitik des WashingtonConsensus geprägt. Bis heute bleiben makroökonomische Verbesserungen aus, die mittels Entwicklungszusammenarbeit erklärt werden könnten (Faust 2010: 41-43).

Die Reduzierung von Armut, verstanden als Finanzarmut, begreift unter Entwicklung lediglich wirtschaftliche Facetten. Amartya Sen erzielte in seiner Studie über Verfügungsrechte (1981) bahnbrechende Erkenntnisse, indem er die Sicht auf die Armen als passive Opfer struktureller Hindernisse (DeHaan \& Zoomers 2003: 3) überwand und zwischen eindimensionaler (Finanz-)Armut und Ver- 
wundbarkeit als komplexem Phänomen unterschied. Entsprechend nutzt diese Arbeit den Begriff der Verwundbarkeit, um Missstände ganzheitlich angehen zu können und sich nicht auf marktwirtschaftliche Denkmodelle begrenzen zu müssen.

Als Startpunkt der Überlegungen bezeichnet Verwundbarkeit die Anfälligkeit gegenüber Gefahren. Darin kommen - als Doppelstruktur betrachtet - zunächst zwei Perspektiven zum Ausdruck. Auf der einen Seite ist man externen Stressoren gegenüber exponiert. Die interne Seite hingegen zielt auf die Bewältigungsmöglichkeiten bzw. deren Mangel ab (Bohle 2001: 3f.). Ist man Gefahren, Stressoren oder negativen Trends ausgesetzt und kann mit diesen nur unbefriedigend umgehen, ist man verwundbar. Das Konzept der sozialen Verwundbarkeit lenkt dabei die Aufmerksamkeit auf bestimmte soziale Gruppen, die eine erhöhte Verwundbarkeit aufweisen. Dies folgt der Erkenntnis, dass bis in die lokale Ebene Verwundbarkeit nicht jeden gleichermaßen betrifft, Gemeinschaften keine homogene Masse bilden, sondern eine Verwundbarkeitsamplitude zwischen einzelnen Personen oder Personengruppen von je gleichen sozialen Merkmalen existiert.

Für eine Annäherung an den Verwundbarkeitsbegriff ist das Modell der Doppelstruktur hilfreich. Bei genauerer Betrachtung wird die Externalisierung von Stressoren der Realität jedoch nicht unbedingt gerecht. Jede Gefahr ist menschgemacht. Naturereignisse wie Dürren, Überschwemmungen, Erdbeben und Vulkanausbrüche werden nur dann zur Gefahr, wenn Menschen sich ihnen aussetzen, und werden erst durch Ungleichheit, Marktversagen oder politischem Versagen zu Hungersnöten (Barnett \& Adger 2007: 641; Sen 1981). Noch deutlicher wird dies bei durch menschliches Eingreifen in die Natur entstandenen Gefahren wie Erosion durch Entwaldung oder Umweltbelastungen und Bodendegradation durch land- und viehwirtschaftliches Missmanagement oder eben dem anthropogenen Klimawandel. Herausforderungen durch politische Spannungen, Bevölkerungsdruck oder Migration sind noch weniger extern zu verankern. Hier muss man eine individuelle oder lokal begrenzte Betrachtungsebene einnehmen, um entfernte Akteure oder großräumige Prozesse zu externalisieren.

Verwundbarkeitsstressoren setzen sich aus verschiedensten Komponenten zusammen. Für diese Arbeit ist es müßig, diese eher der Umwelt, oder eher innergesellschaftlich zuzuordnen. Eine Unterscheidung bzw. Internalisierung und Externalisierung von Gefahren stellt keinen Mehrwert dar, sondern untergräbt stattdessen das hiesige Verständnis, Menschen als Teil der Umwelt bzw. Teil der Natur zu konzeptualisieren. Verwundbarkeit ist allenfalls an der Schnittstelle von Mensch und Umwelt anzusiedeln, jedoch interagieren beide Sphären so stark miteinander, dass die Dichotomie unzulänglich ist.

\subsubsection{Anpassung und Bewältigung}

Menschliches Handeln bzw. deren Unterlassung erzeugt Verwundbarkeit. Menschen sind nicht passive Opfer einer feindlich gesinnten Umwelt, sondern elemen- 
tare Bestandteile der Natur, welche sie aktiv prägen und mitgestalten und lediglich Fehlverhalten kreiert Verwundbarkeit. Auch wenn die Natur dieser Auffassung zufolge keine Gefahr darstellen kann, sondern lediglich Naturereignisse zu sozialen Gefahren werden können, behalte ich sprachlich den Begriff der Naturgefahr bei, um mich im wissenschaftlichen Diskurs über Naturgefahren-vorsorge und Klimawandelanpassung (Birkmann \& Teichman 2009: 6-14; Gero et al. 2010: 8) verorten zu können. Positiv betrachtet wird klar, dass über Veränderungen im menschlichen Handeln Verwundbarkeit überwunden werden kann, Menschen nicht Spielbälle sondern Spieler sind. Solche Handlungen lassen sich sinnvoll in Anpassung und Bewältigung unterscheiden. Bewältigung beschreibt hier die Möglichkeiten mit Herausforderungen umzugehen. Sie konzentriert sich auf Aktivitäten, die während und nach einem Ereignis unternommen werden und ist eine kurzfristige Antwort. Anpassung hingegen bezieht sich auf das Potential, sich den Gefahren nicht nur widersetzen zu können, sondern mögliche Auswirkungen zu verinnerlichen. Langfristige Strategien, die vor, während und nach dem Ereignis angewandt werden, um negative Auswirkungen zu reduzieren, werden unter diesem Begriff vereint (Thywissen 2006: 454). Anpassung bedarf einer Inkorporation der Handlungen als Auslebung der Kultur. ${ }^{26}$ Ein Anpassungsgrad zeigt sich in der Einbettung der Strategien in die Gesellschaft, wobei die Einbettung der Gesellschaft in die Natur eine erfolgsversprechende Grundstrategie scheint. Für soziale Verwundbarkeit bedeutet dies, dass die Anpassungspotentiale gesellschaftlich inhärent sind und sich ihre Stärke in der Fähigkeit zeigt, gemeinschaftlich zu handeln, sodass besonders verwundbare Gruppen nicht ausgeschlossen werden (Adger 2010: 327f.).

Kritische Betrachtungen legen daher nahe, dass eine Anpassung gesellschaftlichen Grenzen unterliege, sofern es gesellschaftsinhärente Einschränkungen gebe (Adger et al. 2009: 337-42). Aufgrund der permanenten Reproduktion, Rekonfiguration und Dynamik, die jeder Kultur innewohnen, sind mögliche soziale Limitationen aus meiner Sicht jedoch überwindbar. Kurz- bis mittelfristig können sich gesellschaftliche Praktiken und Routinen ändern. So hegt diese Arbeit die Hoffnung, zu einer Überwindung des kulturell fest verankerten Eurozentrismus beitragen zu können.

\subsubsection{Resilienz und menschliche Sicherheit}

Als positiven Gegenbegriff von Verwundbarkeit wird oftmals Resilienz gebraucht. Es ist die Eigenschaft einer Gruppe oder eines Systems, einem Schock zu widerstehen und sich aufrecht zu halten. Es beschreibt die Qualität der Absorption, Mitigation, Selbstorganisation und Adaption. Zudem ist es die Fähigkeit sich zu

26 Im Zusammenhang von Naturgefahren plädiert Jörn Birkmann daher für ,the promotion of a culture of disaster resilience" (2006: 9). In dieser Arbeit werden solche Aussagen mit Vorsicht betrachtet: Als Mitglied einer Gesellschaft kann und soll man die eigene Kultur mitgestalten, als Vorwand zur Intervention in fremde Kulturen wäre dies jedoch verwerflich. 
erholen und könnte anhand der Genesungsgeschwindigkeit gemessen werden (Adger 2006: 268-69; Alongi 2008: 2; IPCC 2007b: 86; Kaly et al. 2002: 4; Thywissen 2006: 469). Der Begriff entstammt Konzepten über die Widerstandsfähigkeit von Ökosystemen und ist nun auf menschliche Gesellschaften übertragen worden. In diesem Sinne verstanden ist Resilienz jedoch lediglich re-aktiv. Es ist demzufolge die Fähigkeit zum Ausgangszustand zurückzukehren bzw. in einem stationären Gleichgewicht zu verharren. Resilienz kann daher negative Seiten aufweisen, wenn der status quo problematisch ist. Zur Überwindung von Verwundbarkeit sind jedoch fortlaufende Anpassungen essentiell, die zu einem Wandel der Ausgangslage führen müssen.

Diese Problematik des Resilienzbegriffes ist auch von anderen Autoren erkannt und erste Versuche wurden übernommen, den Begriff neu zu konzipieren. So wird beispielsweise das Konzept als soziale Resilienz erweitert (Keck \& Sakdapolrak 2013), oder aber das Moment der Handlung als entscheidendes Kriterium herausgestellt, wirklich aktiv Verwundbarkeit zu überwinden (Bohle et al. 2009). Die erstgenannte Neukonzeption möchte, als soziale Resilienz verstanden, die Kapazitäten an Anpassung und Bewältigung um Transformationskapazitäten erweitern. Wandel und Krise werden so als der Resilienz inhärente Wesenszüge inkorporiert und weiterhin werden sozio-politische Dimensionen eingearbeitet, um die Bedeutung gesellschaftlicher Aushandlungsprozesse ins Konzept zu integrieren (Keck \& Sakdapolrak 2013.: 10-12). Die zweitgenannte Erweiterung um Handlungsdimensionen kommt zum Ergebnis: „An agency-based framework measures resilience [...] in terms of their [peoples'] human security." (Bohle et al. 2009: 12).

Die Konzeption sozialer Resilienz überwindet entscheidende Schwachstellen des Resilienzbegriffes. Sprachlich präferiert diese Arbeit jedoch, den Ausdruck „soziale Resilienz" nicht zu gebrauchen. Stattdessen nutze ich direkt buman security, menschliche Sicherheit, als Gegenpol zu Verwundbarkeit (Brklacich et al. 2010: 37). Denn menschliche Sicherheit wie ich sie im Folgenden nach Amartya Sen vorstelle, bildet zugleich den konzeptionellen Ausgangspunkt, was unter „gutem Leben“"verstanden werden könne.

\subsection{Menschliche Sicherung durch Verwirklichungschancen}

Der hier verwendete Sicherheitsbegriff grenzt sich bewusst von klassichen nationalstaatlich-territorialen Sicherheitsverständnissen ab. Er stellt menschliche Individuen in den Fokus. Zu Generierung menschlicher Sicherheit steht nicht die Förderung von Anpassungshandlungen im Fokus, sondern die Schaffung von Handlungspotentialen bzw. nach Amartya Sen „Verwirklichungschancen“ (1979: 217-219), negative Auswirkungen möglicher Gefahren zu reduzieren oder ganz zu vermeiden. Individuelle Verwirklichungschancen generieren sich dabei nicht nur aus materiellen Gütern, sondern fußen ebenso auf der körperlichen wie geistigen Verfassung des Akteurs. Insbesondere aber sind Zugangsmöglichkeiten zu Aktiva und Institutionen, und damit gesellschaftliche Komponenten entscheidend für die 
eigene Verwirklichung zur Absicherung gegenüber Verwundbarkeiten. Maßgeblich sind Rechte und Freiheiten, die zu eigenverantwortlichem Handeln frei von sozialen Barrieren befähigen und aufmuntern. Die Basis, auf der Handlungen zur individuellen wie gesellschaftlichen Verwirklichung stattfinden können, bilden Zugang zu und Mitbestimmung von gesellschaftlichen Strukturen und Prozessen, angelegt sowohl in institutionellen, politischen, rechtlichen, ökonomischen und kulturellen Sphären, als auch auf räumlichen und zeitlichen Skalen. Nicht nur die individuelle Ausstattung, sondern auch die Zugangsmöglichkeiten, diese Aktiva sinnvoll verwenden zu können, bestimmen die Befähigung zur Selbstverwirklichung.

Verwirklichungschancen richten sich nicht nur auf die Reduzierung von Verwundbarkeit, sondern zielen aktiv auf die Befähigung, ein erfülltes Leben zu gestalten. Das Konzept menschlicher Sicherheit weist den Weg für ein gutes Leben in Selbstrespekt und Würde, mit Glück und Wohlbefinden. Lebensqualität stellt hierfür den Oberbegriff dar.

Im Sinne der Commission on Human Security (CHS), geleitet von Sadako Ogata und Amartya Sen, versteht sich das Konzept menschliche Sicherheit als die Kombination von drei verschiedenen Freiheiten: Freiheit von Not, Freiheit von Angst und Freiheit zu selbstbestimmtem Handeln (CHS 2003: 10-11). Die ersten beiden Typen werden durch die Gewährleistung der Grundbedürfnisse sowie dem Schutz vor Gewalt geschaffen, letzterer durch empowerment. Im Gegensatz zu klassischen nationalstaatlichen und verkürzten individuellen Sicherheitsverständnissen beschränkt er sich nicht auf passive Freiheitsrechte („Freiheit von“), sondern schließt das aktive Freiheitsrecht („Freiheit zu“) ein. Dieser Freiheitsbegriff wird zudem sehr ernst genommen: man hat die Freiheit zu handeln, jedoch besteht kein Zwang, tatsächlich Handeln zu müssen. Entsprechend zielen Verwirklichungschancen einzig auf die Befähigung zu Handeln ab; ob oder wie stark diese Möglichkeiten ausgeschöpft werden, steht dem Akteur frei. Ähnlich ist empowerment als Selbstermächtigung ein innerer Prozess, von außen kann lediglich ein Beitrag dazu geleistet werden, den Prozess zu unterstützen. Das hiesige Sicherheitsverständnis reduziert sich demnach bewusst nicht auf eine Abkehr und Kontrolle von Bedrohungen bzw. Verwundbarkeiten, wie es international seit den Terroranschlägen vom 11. September 2001 zunehmend Verwendung findet (Klingebiel 2015: 17). Im Gegenteil hat menschliche Sicherheit vielmehr das aktive Freiheitsrecht zur individuellen Selbstverwirklichung im Sinne.

Ein gewalt- und angstfreies, selbstbestimmtes Leben, gekennzeichnet durch einen Freiraum zur offenen und aktiven Lebensgestaltung eröffnet demnach ein Leben in Würde. Als soziale Wesen verstanden, werden Menschen dabei ihre Freiheiten auch dazu nutzen, in Gesellschaft zu leben, zu interagieren, kooperieren und dabei Konflikte zu lösen. Menschenwürdige Entwicklung hat diese Freiheitskonzeption im Sinn. Diese Arbeit hat sich im Zuge des partizipativen Dialoges diesen Idealen von Freiheit und Selbstverwirklichung verschrieben, sodass nicht aktiv Handlungen eingefordert werden. Stattdessen soll lediglich ein Betrag dazu geleistet werden, Rahmen und Strukturen so zu verändern, dass Handlungsmög- 
lichkeiten zur Selbstverwirklichung erweitert werden; bzw. so zu erhalten, dass bestehende Handlungsmöglichkeiten nicht wegfallen.

Auch ist diese Arbeit eindeutig menschzentriert und dabei akteursorientiert. Akteursorientierung bedeutet, dass die Handlung des Akteurs im Vordergrund steht. Im Gegensatz zu akteurszentrierter Forschung werden dabei Rahmen und Strukturen, in denen die Handlung eingebettet ist, berücksichtigt, sodass die Dichotomie von Struktur und Handlung überwunden wird. Pierre Bourdieu führte als Vermittlungsfunktion den Habitusbegriff ein (1983b). Ein Individuum verinnerlicht kollektive Einstellungen, sodass in den Handlungen des Individuums Kollektives in Form von Kultur wiederzufinden ist. Durch die individuelle Einverleibung gesellschaftlicher Strukturen werden im Habitus diese Strukturen einerseits permanent reproduziert, andererseits erst generiert. Ähnlich rückte Anthony Giddens Theorie der Strukturierung (1988) Individuen in den Mittelpunkt, die nicht nur routiniertes Alltagsverhalten kennzeichnet, sondern - wenn auch begrenzt - ihre Aktivitäten und strukturellen Rahmenbedingungen an einem spezifischen Ort reflektieren und über gezieltes Handeln aktiv verändern. Die Handlung des Subjektes steht in Relation zur Gesellschaft. Durch die Interpretation ihres sozialen Raumes erfahren die Individuen einen gesellschaftlichen Handlungssinn (Dörfler et al 2003: 15-18), wobei Handeln selbstbestimmt zweckorientiert ist (Tröger 2003: 27). Die Strukturen sind dabei rekursiv in soziale Systeme einbegriffen und durch einen Dualismus gekennzeichnet, wonach einerseits die Strukturen das Handeln produzieren, andererseits durch dieses reproduziert und verändert werden. Die Struktur schränkt daher Handeln nicht nur ein, sondern ermöglicht es auch und stellt demnach keinen Zwang dar (Giddens 1988: 78f.). Strukturelle Rahmenbedingungen werden im Sinne der Strukturierung vielmehr als Angebot denn als Hindernis aufgefasst und schaffen Handlungsfreiheiten (Giddens 1988: 215, 227) - also die Befähigung zu handeln. Der Erkenntnis folgend, dass Strukturen einer Wahrnehmung und Interpretation bedürfen, hegt diese Studie methodologisch - ganz im Sinne der Hermeneutik - keinen Anspruch absoluter Objektivität; die Realität unterliegt der Wahrnehmung des Forschers, also meiner Interpretation im Zusammenspiel mit den weiteren Beteiligten.

Diese Arbeit möchte daher nicht nur die Struktur menschlicher Sicherheit betrachten, also den Zustand menschlicher Sicherheit beschreiben. Vielmehr möchte ich darüber hinaus die Handlungen analysieren, die diesen Zustand beeinflussen. Im Fokus steht Sicherung, nicht Sicherheit. Da Menschen soziale Wesen sind, beinhaltet menschenwürdige Entwicklung gesellschaftliche Komponenten. Losgelöst vom sozialen Umfeld ist das erwünschte, menschlich sichere Leben nicht zu erreichen. Lebensqualität äußert sich demnach in der Befähigung zu sozialer Sicherung.

Aus einer psychosozialen Perspektive ist Sicherheit ein Gefühl. Sie unterliegt subjektiver Wahrnehmung. Gefahren beeinflussen das Sicherheitsgefühl nur, wenn die jeweilige Gefahr antizipiert wird, also zum Risiko wird (Beck 2007: 29). Vor dem 11. September 2001 waren sich Fluggäste sicher, dass ihr Flugzeug nicht 
als Waffe für ein Selbstmordattentat missbraucht wird, da dieses Gefahrenpotenzial nicht in Betracht gezogen wurde. Heute ist diese Gefahr wahrgenommen und durch die omnipräsenten Sicherheitskontrollen an Flughäfen wird das Risiko ins Bewusstsein eingebrannt, sodass man sich unsicherer fühlt, obwohl die Gefahr minimiert wurde. In der heutigen, globalisierten Wissensgesellschaft leben wir in dem Paradox, dass die weltweite Aufklärung über Gefahren in kognitiver Hinsicht nicht zur Sicherheit, sondern zur Unsicherheit beiträgt.

Nur im Zusammenspiel mit den Mitmenschen ist Sicherung möglich. Menschliches Leben kann - und das nicht nur aus rein biologischen Gründen - nur in Gemeinschaft fortbestehen. Sowohl kollektiv als auch individuell ist das subjektive Gefühl von Vertrauen anstelle von Angst, zu schaffen durch Interaktion, Kooperation und Reziprozität, zur Sicherung entscheidend. Ich spreche daher von sozialer Sicherung als Schlüssel für Wohlbefinden und Lebensqualität. Diese Studie überprüft, inwieweit eine gesellschaftliche Transformation, die das Vordringen eines marktwirtschaftlichen Konkurrenzgedankens kennzeichnet, soziale Sicherung beeinträchtigt. Im Zentrum stehen Menschen der Fidschi-Inseln. Darüber hinaus werden solche Handlungsstrategien vorgestellt, die Beeinträchtigungen minimieren oder gar ins Gegenteil verkehren.

\subsection{Ein integratives Konzept zu sozialer Sicherung}

Diese Arbeit bricht mit vielen klassischen Grundanschauungen der Wissenschaftswelt. Dichotome Denkkategorien wie Mensch-Natur, IndividuumKollektiv, Handlung-Struktur, Wirtschaft-Politik-Gesellschaft, sowie methodologisch, Forscher und Beforschter bzw. Laie und Experte werden aufgelöst. Den subjektiven Empfindungen der Partizipanten der Studie wird mehr Gewicht eingeräumt als „harten“ Zahlen und Fakten. Dies geschieht, weil die Lebensrealität der Menschen vor dem Hintergrund gesellschaftsinhärenter kultureller Werte betrachtet wird. Letztere können sich wesentlich besser über Narrative, also idealisierten Erzählungen, darstellen lassen, als anhand von demographischem oder ökonomischem Zahlenmaterial. Oftmals nehmen Menschen die Welt anders wahr und kommen zu anderen Schlüssen und Handlungen, als klassische wissenschaftliche Perspektiven vermuten ließen.

Eine Beschreibung von Lebenssituationen ist belanglos, solange Maßstäbe fehlen, anhand derer die Qualität dieser Lebenslage bestimmt wird. Solche sind jedoch sozio-kulturell bestimmt. Die zentralen Erkenntnisse dieser Studie sind daher nicht quantifizierbar, sondern sie unterliegen stattdessen intersubjektiver Wahrnehmung.

\subsubsection{Wertebasierter Ansatz}

Die Frage, wie man mit gesellschaftlichen Transformationsprozessen umgeht, wird anhand von Werten beantwortet. Welche Prinzipien sind von intrinsischem Wert, welchen Blickwinkeln unterliegen diese und wie sollen Anpassungsmaß- 
nahmen entsprechend dieses Wertekodexes aussehen? Eine Messbarmachung bzw. Monetarisierung von Werten trifft nicht den Kern der Frage. Das Leben, ob menschlich, pflanzlich oder tierisch, hat heilige Dimensionen und kann und darf keinen Marktprinzipien untergeordnet werden. ${ }^{27}$ Große Signifikanten wie - in eurozentrischer Sprache ausgedrückt - Freiheit, Gerechtigkeit, politische Mitbestimmung, genauso aber auch Nachhaltigkeit, Nächstenliebe, Vertrauen und Respekt sind alle von intrinsischem, immateriellem Wert und bedürfen einer Konsistenz in Zeiten des gesellschaftlichen Wandels. Weitere Werte werden über die Narrative als stetige Werteinterpretationen behandelt, sodass nicht auf bloße Zahlen, sondern vielmehr auf Geschichten, Mythen und Legenden, sowie Ritualen und alltäglich gelebter Praxis zurückgegriffen wird. Somit werden möglichst ganzheitlich die für die Lebensqualität der lokalen Bevölkerung ungemein wichtigen Dimensionen ihres alltäglichen Lebens partizipativ in diese Studie integriert. Dieser transdisziplinäre Charakter der Arbeit versucht damit, der Forderung nach einem integrativen, wertebasierten Ansatz (O’Brien \& Wolf 2010; Ukowitz 2014) gerecht zu werden.

Die zu behandelnden Narrative stellen Ideale dar, die in Reinform nicht existieren. Somit werden wahrgenommene „ist“-Zustände dargestellt, deren Qualität auf der Annäherung an ideale „soll“-Zustände ${ }^{28}$ sowie dem Vergleich von ,ist"Zuständen basiert. Werte unterliegen Aushandlungskonflikten. Ihre Priorisierung erfolgt individuell, wenn auch beeinflusst von gesellschaftlichen Strukturen. Ferner existiert kein die gesamte Gesellschaft abdeckendes Ideal und es wird nicht angestrebt, ein solches zu kreieren. In einer Gesellschaft bedarf es jedoch einer Kommunikation, mittels derer Werte und Normen, Entwicklung und sonstige Ideale ausgehandelt werden. Zur Bewertung von Gerechtigkeitszuständen benötigt es dabei allerdings keines allgemeinen Gerechtigkeitsideals. Denn mittels des Vergleichs können sehr wohl Unterschiede festgestellt werden. Als Maßstäbe können Werte herhalten, die vernünftig als sinnvoll und nachvollziehbar verstanden werden können, sodass Komparatistik zur Bewertung von Gerechtigkeitszuständen hinreicht (Sen 2010: 44-46).

\subsubsection{Demokratie als öffentlicher Vernunftgebrauch}

Gemäß der Annahme pluraler Ansichten innerhalb menschlicher Gruppen existiert ein permanenter Konflikt über Werte. „Public reasoning“ bzw. „Öffentlicher

\footnotetext{
${ }^{27}$ Dies ist nicht bloß die Vorwegnahme eines Ergebnisses der Empirie, als dass die Natur im fidschianischen Kollektiv einen intrinsischen Wert besitze. Gestützt wird diese These auch dadurch, dass selbst in Mitteleuropa der Natur eine Würde zugeschrieben wird, beispielsweise in der Schweiz verfassungsrechtlich verankert als „die Würde der Kreatur“, wobei „Kreaturen“ als „Tiere, Pflanzen und andere Organismen" spezifiziert werden (Sitter-Liver 2012: 376). Am prominentesten ist der intrinsische Wert der Natur allerdings wohl in der ecuadorianischen Verfassung verankert (Arsel 2012; Salleh 2010: 216).

28 Aufgrund der unterstellten Inkohärenz innerhalb kultureller Gesellschaften wird im Plural gesprochen.
} 
Vernunftgebrauch" steht im Zentrum der Konfliktlösung und sozialen Sicherung (Sen 2004a: 53f., Sen 2010). Zur gewaltfreien, menschenwürdigen Aushandlung von Konflikten bedarf es der Möglichkeit, öffentlich eigene Meinungen äußern und begründen zu können. Weiterhin bedarf es des Willens der Adressaten zuzuhören und des Versuchs, vorgetragene Argumente nachzuvollziehen. Auf dieser Grundlage können, sofern erforderlich, Gegenargumente vorgetragen und begründet werden, welche ebenfalls auf eine offene Zuhörerschaft angewiesen ist. Eine barrierefreie Kommunikation, welche wiederum auf gegenseitiges Vertrauen und einem Wunsch nach sozialer Nähe und Kooperation fußen kann, stellt die Grundlage einer öffentlichen, vernunftgesteuerten Diskussion zu menschlich sicherer Konfliktlösung dar. In Anlehnung an Amartya Sen stellt diese Arbeit die These auf, öffentlicher Vernunftgebrauch sei der Kern des Ideals der Demokratie (Sen 2010: 347-364).

Auch wenn es komplexe Verständnisse von Demokratie gibt, so reichen für eine staatstheoretische Minimaldefinition und eine gleichzeitige Abgrenzung zu Nicht-Demokratien zwei Kriterien aus: ,public contestation and the right to participate" (Dahl 1971: 5). Anders formuliert müssen ein aktives und passives Wahlrecht, freie und faire Wahlen, Assoziations- und Koalitionsfreiheit, Konkurrenzkampf sowie Informationsfreiheit gegeben sein (Merkel 1999: 31). Sind diese Kriterien nicht komplett erfüllt, so kann ein Regime nicht als „reine“ Demokratie gelten sondern ist allenfalls eine „defekte“ oder „unvollständige“ Demokratie. Sie ist ein „hybrides Regime“ oder „Grauzonenregime“ zwischen Demokratie und Autokratie (Merkel et al. 2003: 30f.). Sind die Kriterien völlig beschädigt oder finden gar keine Wahlen statt, so ist das Regime entsprechend als Autokratie zu bezeichnen. ${ }^{29}$

Machttheoretisch hingegen lässt sich Demokratie politisch definieren als Herrschaft aller bzw. Herrschaft des Volkes. Wer oder was der demos bzw. das Volk ist, ist jedoch unklar. Der Volksbegriff stellt einen leeren Signifikanten dar, der sich nahezu beliebig füllen lässt (Gießen \& Seyfert 2013: 41f.). Nach heutigem Verständnis dürfen in der Regel nur erwachsene Staatsbürger partizipieren. Doch das Ausmaß der Partizipation ist auch in anderer Hinsicht begrenzt: „Der Wille der Mehrheit stößt dann auf Grenzen, wenn er Grundlagen der Demokratie selbst missachtet. So stehen die Menschenrechte nicht zur Disposition der Mehrheit.“ (Lauth 2002: 111.) Die Demokratie als Herrschaft aller mit uneingeschränkter Volkssouveränität ist also ein Idealbild, das real nicht existiert.

Den staats- und machttheoretischen Begriffsdimensionen von Demokratie gemein ist das Recht ihrer Mitglieder auf Partizipation am gesellschaftlichen Leben. Freiheit zu öffentlichem Vernunftgebrauch ist damit nicht nur die Grundlage, sondern zugleich die Form der praktischen Auslebung der Demokratie. Öffentli-

\footnotetext{
${ }^{29}$ Nach altertümlichem Verständnis ist bereits die Demokratie - neben anderen - eine verfehlte Herrschaftsform; „,die Demokratie [sei] eine solche zum Nutzen der Armen. Keine aber denkt an den gemeinsamen Nutzen Aller.“ (Aristoteles ca. 335-323 v. Chr. /2004: 55).
} 
cher Vernunftgebrauch ist daher ein elementares Freiheitsrecht zu sozialer Sicherheit. Anstelle eines westlichen Demokratieverständnisses als gesellschaftlichen Wert werde ich von nun an sprachlich von öffentlichem Vernunftgebrauch reden, um eurozentrische Weltbilder und Wertvorstellungen zu vermeiden und offener kulturelle Ideale nach gewaltfreier, gesellschaftlich akzeptierter Konfliktlösung benennen zu können.

Die in diesem Abschnitt dargelegte wertebasierte theoretisch-konzeptionelle Rahmung vervollständigt den integrativen, partizipativen und ergebnisoffenen theoretischen und methodologischen Ansatz. Um die Qualität menschlichen Lebens aufrecht zu halten, oder die Transformation als Chance zu nutzen, Lebensqualität zu steigern, müssen die Narrative unter den sich verändernden Rahmenbedingungen neu erzählt, neu ausgehandelt werden. Aushandlungsprozesse bedürfen einer Interaktion im Sinne eines öffentlichen Vernunftgebrauches. Über solch einen breiten, wertebasierenden und integrativen Ansatz können die Lebensrealität sinnvoll erfasst und gesicherte, qualitative Schlussfolgerungen gezogen werden (O’Brien \& Wolf 2010). 



\section{Kulturräumlicher Überblick}

Wie nahezu jede sozialwissenschaftliche Studie besitzt auch diese eine geschichtliche Dimension, da die getätigten Aussagen nicht losgelöst werden können vom Kontext, in welchem die Erkenntnisse erzielt wurden (Lund 2014: 225). Dieser Abschnitt legt daher das historische Fundament, auf dem die Studie basiert. Gleichzeitig hat diese Studie einen regionalen Fokus. Wie in den vorherigen Abschnitten erörtert, liegt dem Begriff eines Kulturraumes dabei kein statisches und geschlossenes Verständnis eines Containers zugrunde. Bestimmte Identitätsmerkmale, Sinn- und Symboldimensionen können jedoch kulturell und materiell räumlich verortet werden, auch wenn Grenzen sich überlappen und fließend sind. Ein - wie auch immer gearteter - ganzheitlicher Überblick über den Kulturraum ist demnach nicht möglich. Stattdessen werden zielgerichtet Aspekte der Kultur und ihrer Historie aufgegriffen, welche für das Verständnis der heutigen Lebenswelt der Fidschianer, ihrer Werte und Wünsche, von Bedeutung sind.

Diese Herangehensweise stellt keinen Versuch dar, gesellschaftliche Dimensionen zu verschleiern, welche die Aussagen der hiesigen Studie konterkarieren würden. Im Gegenteil strebt dieser Abschnitt eine ausgewogene Mischung an, einerseits einen möglichst weitgefächerten Überblick über das Untersuchungsgebiet zu geben um der Leserschaft Möglichkeiten zu geben, eigenständig und informiert die örtlichen Lebensrealitäten zu interpretieren. Andererseits soll diese Studie nicht ausufern, sondern in diesem Abschnitt stringent den ethnohistorischen Hintergrund skizzieren, mit Hilfe dessen das Leitthema der Arbeit gesellschaftliche Transformation - aufgeworfen wird und begründet wird, warum dies treffend als Spannungsfeld charakterisiert werden kann. Dies geschieht in erster Linie aus struktureller Perspektive über eine Diskussion mit der Fachliteratur. An geeigneten Stellen werden die Aussagen allerdings mit empirischen Erkenntnissen unterfüttert. 



\section{Fidschi im Zeichen gesellschaftlicher Transformation}

Fidschi liegt im Südpazifik und besteht aus mehreren hundert Inseln. Kulturräumlich unterscheidet man die pazifische Inselwelt grob in Melanesien, Polynesien und Mikronesien. Fidschi liegt im Grenzgebiet zwischen Melanesien und Polynesien. Betrachtet man die Pazifikstaaten abzüglich Papua Neuguinea und Hawaii, so stellt Fidschi mit ca. 900.000 Einwohnern mehr als 25\% der pazifischen Gesamtbevölkerung. Der Agglomerationsraum um die Hauptstadt Suva bildet mit ca. 250.000 Einwohnern die einzige pazifische Großstadt mit sechsstelliger Einwohnerzahl (SPC 2013). Fidschi nimmt eine Primatstellung im Pazifik ein.

Dieses Kapitel startet mit einem pointierten Abriss der Geschichte Fidschis, der sowohl auf Erkenntnissen von Archäologen und Historikern, als auch auf Entstehungsmythen fußt. Dank der gewählten Erzählform der Geschichte lassen sich so bereits kulturelle Werte ableiten. Zur fundierten Erfassung der Lebenssituation fidschianischer Küstendorfbewohner wird anschließend der Naturraum des heutigen Staatsgebiets mit Blick auf Klimawandel und Naturgefahren skizziert. Zum Schluss des Kapitels stehen aktuelle gesellschaftliche Trends und Dynamiken im Zentrum, welche schlussendlich als Transformationsprozess bewertet werden. Hierbei wird klar, dass der Wandel sowohl Risiken birgt, als auch Chancen eröffnet. Der Umgang der Bevölkerung mit diesen wird in den nachfolgenden EmpirieKapiteln lokalspezifisch analysiert.

\subsection{Historischer Abriss Fidschis}

Die genaue Abfolge der Erstbesiedlung vieler Pazifikinseln ist bis heute ungeklärt. Die Erstbesiedlung der Fidschi-Inseln erfolgte wahrscheinlich vor rund 3.500 Jahren von Westen her. Nach einer melanesischen Erklärungsvariante kamen Seefahrer aus Neu-Guinea und den Salomonen nach Fidschi. Eine polynesische Variante legt die Besiedlung der Inselregion um die heutigen Staaten Fidschi, Samoa und Tonga von Taiwan und den Philippinen her nahe. Diese Schätzungen beruhen maßgeblich auf Ausgrabungen von Keramiken, welche sowohl mittels 14C-Methode als auch über eine Einbettung der Fundstücke in die Historie der Keramik-Kunst - Lapita genannt - des gesamten Südpazifikraums datiert werden (Nunn 1998: 230-236). Von dieser Inselregion aus bereitete sich eine polynesische Kultur weiter nach Osten aus, sowie um 400n.Chr. nach Norden bis Hawaii und um 1000n.Chr. nach Süden bis Neuseeland. Eine frühe Prägung Fidschis durch Polynesier ist zudem über Sprachverwandtschaften offensichtlich, zudem halten sich gerade auf den östlichen Inseln bis heute für Polynesien typische Häuptlingssysteme. 
C: Kulturräumlicher Überblick

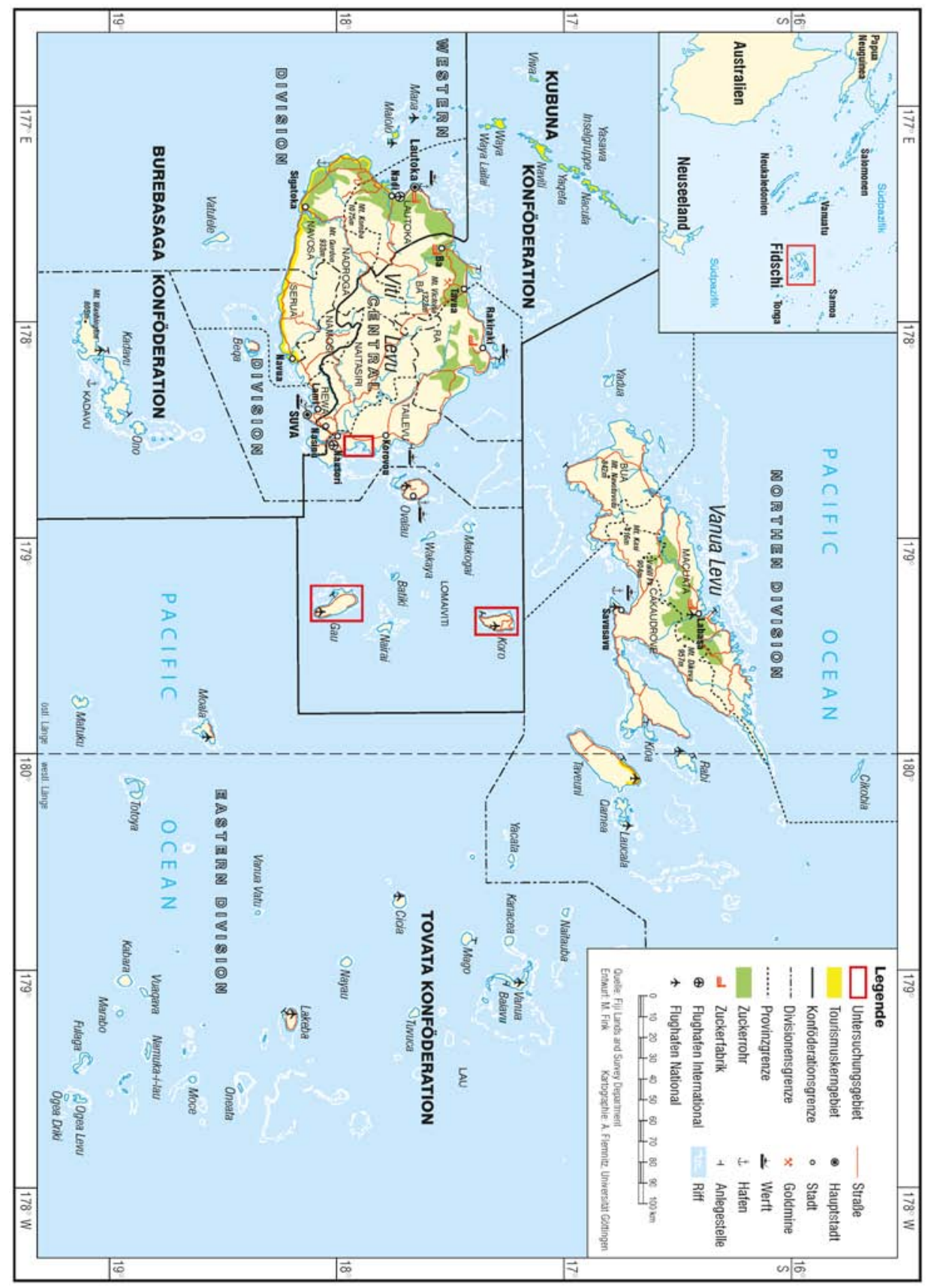

Karte 1: Übersichtskarte Fidschi 
Neben der Besiedlungsvariante über Taiwan besteht die Theorie einer langsameren Wanderung vom Asiatischen Kontinent aus über Indonesien und die melanesischen Inseln Neuguinea, Salomonen und Vanuatu nach Fidschi. So unterliegen die westlicheren Inseln Fidschis sowie die Westseite der Hauptinsel Viti Levu stärker melanesischen Einflüssen mit kleinräumigeren Ordnungsprinzipien. Eine weitere Besiedlungsvariante des Südpazifiks von Amerika gilt zwar als unwahrscheinlich, ist jedoch theoretisch möglich. So baute Thor Heyerdahl ein Floß auf Basis des Wissensstandes amerikanischer Ureinwohner und segelte vom heutigen Peru aus mit der Humboldtströmung nach Polynesien.

Die Dreigliederung in melanesische, polynesische und mikronesische Kulturen wurde Ozeanien im 19. Jahrhundert seitens der Europäer aufgesetzt. Inzwischen hat sie das kulturelle Bewusstsein der Inselbewohner durchdrungen. Da sie zur Beschreibung Fidschis jedoch nur bedingt hilfreich ist (Nunn 2007: 31), spreche ich lieber allgemein von Ozeanien oder spezifiziere räumlich den Südwestpazifik. Festzuhalten ist, dass die Inseln wohl kaum zufällig erstbesiedelt wurden, wie manche, von imperialistischen oder neokolonialen Denkweisen in die Irre geführte Beschreibungen suggerieren (Burns 1963: 25). Vorstellungen wurden verbreitet, Fischer seinen beispielsweise womöglich vom Kurs abgekommen - Wie es der Zufall will glücklicherweise samt Frauen, Kindern, Haustieren und Samen? -, seien auf unbekannten Inseln gestrandet und hätten sich in Isolation eine neue Heimat aufgebaut, aus der ein neues Volk entstand. Stattdessen standen die Menschen der heutigen Fidschi-Inseln wohl lange vor Ankunft der ersten Europäer in weitreichenden Austauschbeziehungen mit Samoa, Tonga, Rotuma, Niue, Tokelau, Tuvalu, Futuna und Uvea; weiterhin gelangten die Seefahrer aufgrund ihrer Fähigkeiten bis Kiribati, den Solomonen, Vanuatu und Neu Kaledonien, sowie schlussendlich in der Nord-Süd-Ausdehnung bis Hawaii und Neuseeland (Hau'ofa 1993: 9). Fasst man Ozeanien als einen zusammenhängenden Raum auf, so erstreckt sich dieser über rund 40\% der Erdoberfläche und ist entsprechend reich und divers an kulturellen Errungenschaften. Dieser Reichtum drückt sich unter anderem in kulturellem Wissen und Erfahrungen aus, verarbeitet in Mythen und Legenden.

\subsubsection{Entstehungsmythos}

Nach der in Fidschi am weitesten verbreiteten Entstehungslegende - einer Pflichtlektüre im Schulunterricht (Reed \& Hames 1967) - landete Degei, der Hauptgott der Fidschianer aus dem das fidschianische Volk hervorging, gemeinsam mit Lutunasobasoba an der Westküste Viti Levus und gründete dort die Siedlung Vuda („unser Ursprung“). Diese Siedlung wurde jedoch aufgegeben und Degei zog nach Nakauvadra im Nordwesten Viti Levus. Degei wird mal in Menschengestalt dargestellt, mal in Form einer Schlange oder formlos als Geistgestalt. Im Zorn verursachte er einst eine große Flut, welche seine Nachfahren über ganz Fidschi verbreitete. Lutunasobasoba und Degei kamen ursprünglich aus dem Theben des 
heutigen Ägyptens, segelten den Nil aufwärts und vom heutigen Tansania aus nach Fidschi.

Die heute bekannteste Version um Degei gewann 1892 ein Preisausschreiben zur Einsendung von Entstehungsgeschichten einer fidschianischen Zeitung. Zu dieser Zeit existierten hunderte weitere, teils voneinander unabhängige Entstehungsmythen, manche halten sich bis heute. Seit Mitte des 19. Jahrhunderts wurde aus kolonialistischen und nationalistischen Motiven heraus eine Entstehungslegende gesucht, die Fidschianer als Nationalvolk zu einen. Geschrieben wurde sie von einem Absolventen einer christlichen Missionsschule (Gravelle 1988: 6f.). Der hybride Charakter der Kultur und die weitreichende Nachwirkung einer Kolonialisierung auf eine traditionelle Kultur werden hier besonders deutlich. Die heute in der breiten Bevölkerung akzeptierte Entstehungslegende über den Ursprung der fidschianischen Kultur, also ein Kernelement des Narrativs, wo traditionelle Werte herrühren und was die eigene Identität ausmacht, entstand erst in Retrospektive und aus kolonialistischen und christlichen Motiven heraus.

Genauso hebt der Entstehungsmythos auch die Wichtigkeit hervor, Tradition als einen Prozess zu verstehen. Da der christliche Glaube über die Zeit zu einem Fundament im Selbstverständnis der Fidschianer geworden ist (Toren 1988: 697f.), macht gerade die Vereinbarkeit mit dem christlichen Glauben die Stärke dieser Legende aus. Sie wirkt identitätsstiftend, über die Verortung der Ahnen in Theben wird ein historischer Bezug zur Welt des Alten Testaments konstruiert. Viele Fidschianer erfreuen sich an der so entstandenen Möglichkeit, sich gar als verlorener Stamm Israels zu sehen (Vielzahl informeller Gespräche).

Dadurch, dass traditionelles geschichtliches Wissen bis zur Einführung der Schrift seitens der Europäer auf mündliche Überlieferungen beruhte, konnten Entstehungsmythen besonders gut im Zeitgeist so erzählt werden, dass sie stets angepasst einen Mehrwert zum Verständnis gegenwärtiger Strukturen beitrug. Insbesondere mündliche Überlieferungen helfen dem Selbstverständnis und der sozialen Verortung kultureller Gemeinschaften und ihrer individuellen Mitglieder; jedoch sind sie im Vergleich zu schriftlichen Überlieferungen besonders anfällig für politisch motivierte Manipulation. Dieses Kapitel stellt daher weniger den Versuch dar, die Geschichte Fidschis „objektiv“ tatsachenbasiert darzustellen, sondern vielmehr Elemente herauszuarbeiten, die in meinen Untersuchungsdörfern praktisch genutzt werden. Deutlich spürbar ist der europäische Imperialismus und seine Nachwirkungen bis in die heutige Zeit; über die Zeit hinweg konnten jedoch oftmals Handlungsmuster etabliert werden, die es ermöglichen, die weitreichenden Veränderungen in die örtlichen Lebensweisen zu integrieren, sodass Schäden abgewendet werden konnten und sich der Wandel punktuell sogar positiv auf die Lebensqualität niederschlug. 


\subsubsection{Präkoloniale Ära}

1643 wurden mehrere Fidschi-Inseln von Abel Tasman gesichtet, 1774 betrat sie James Cook. Nach der Meuterei auf der Bounty durchquerte Willam Bligh 1789 notgedrungen fidschianische Gewässer in seiner Barkasse auf dem Weg nach Timor. Seit Anfang des 19. Jahrhunderts ließen sich vermehrt zwielichtige Europäer als Strandpiraten, Farmer und Händler insbesondere an den Küsten Viti Levus und Ovalaus nieder. Seither stehen die Fidschianer in Kontakt mit Europa. Erste wichtige Handelsgüter waren Sandelholz und Seegurken nach China, bevor Mitte des Jahrhunderts Baumwollplantagen errichtet wurden (Burns 1963: 55, 77f.; Gravelle 1988: 42, 147f.; Weber 2007: 208). An den eingeschleppten Krankheiten, zu Beginn vor allem die Ruhr, verstarben viele Fidschianer. Insbesondere die mangelnden Heilungserfolge seitens der Priesterklasse boten einer christlichen Missionierung Chancen (Scarr 1984: 9-15).

Die ersten christlichen Missionare landeten 1835 auf Lakeba in der LauInselgruppe und hatten in den Anfangsjahren Anlaufschwierigkeiten. Weitere semi-erfolgreiche Missionsstationen folgten kurze Zeit später, unter anderem auf der Hauptinsel Viti Levu in Rewa oder auf Taveuni. Reverend John Hunt etablierte schließlich ab 1842 erfolgreich die methodistische Kirche auf Viwa. 1845 wurde von ihm der erste Häuptling missioniert und 1847 das Neue Testament in den Bau-Dialekt des Fidschianischen übersetzt, welcher so zur Schriftsprache avancierte (Burns 1963: 57-64; Gravelle 1988: 53-55).

Die Fidschianische Gesellschaft war zu dieser Zeit in verschiedene Stammesverbände unterteilt, die sich oftmals feindlich gegenüberstanden. Über Heiratsallianzen verbanden sich mehrere Herrscherfamilien, andere wurden durch kriegerische Auseinandersetzungen unterworfen. In den östlichen Inseln bildeten sich drei Stammeskonföderationen heraus, Tovata, Kubuna und Burebasaga, die das Gebiet des heutigen Fidschis unter sich aufteilten. Sie wurden von ParamountHäuptlingen geführt. ${ }^{30}$ Viwa liegt strategisch günstig östlich Viti Levus vorgelagert und benachbart zu Bau Island, dem politischen Zentrum der KubunaKonföderation. Ihr Häuptling, Ratu Seru Epenisa Cakobau, entsagte unter dem Einfluss von Viwa dem Kannibalismus und konvertierte 1854. Bereits in den 1870er Jahren war die Missionierung weiter Bevölkerungsteile Fidschis abgeschlossen, jedoch ohne die alten Glaubensvorstellungen dabei zwingend zu verdrängen. Vielmehr wurden diejenigen christlichen Praktiken und Glaubensvorstellungen übernommen, die mit den bisherigen zusammenfielen - beispielsweise dass Engel bzw. Geister Verstorbener existieren und Gottesdiensten beiwohnen (siehe Kap. 7) - und die Konvertierung wurde von der Hoffnung genährt, dass der

\footnotetext{
${ }^{30}$ Es ist unklar, wie sehr ein Vasallenstatus im Alltag spürbar war, da Inselreiche aufgrund mangelnder Kontrollinstrumente und logistischer Hindernisse schwer zu beaufsichtigen waren. Gelegentlich wurde die Treue jedoch durch Abgaben maritimer und agrarische Handelsgüter sowie dem Bereitstellen von Kriegern eingefordert (Bayliss-Smith 1976). Zweifelsfrei war die sozio-politische Macht der Stammeshäuptlinge am jeweiligen Wohnsitz am größten.
} 
christliche Gott die neuen, unbekannten Krankheiten heilen könne (Scarr 1984: 12-21).

Cakobaus Vorgänger als Häuptlinge von Bau boten bereits europäischen Strandräubern Unterschlupf. Auch Dank deren Schusswaffen erhob sich die Konföderation Mitte des 19. Jahrhunderts kriegerisch zu einer Vormachtstellung innerhalb Fidschis (Burns 1963: 51-56), wobei die traditionellen Wege der Bündnissuche über Heiratsallianzen strategisch bedeutender waren (Scarr 1984: 11f.). Die ausartenden Kriege zwischen und innerhalb der Konföderationen hatten einen staatsbildenden Charakter für die fidschianische Gesellschaft (Weber 2007: 208f.). Nicht zuletzt durch seine Konvertierung intensivierte Cakobau die Beziehungen zu Europa und den USA, und zeigte hierbei besonderes Handelsgeschick über Kriegswaffen. 1871 ließ er sich zum Tiu Viti (König von Fidschi) krönen, ein zuvor nicht existierender Titel, der außerhalb des Einflussgebietes Baus fidschiweit kaum auf Anerkennung stieß, jedoch seitens der imperialistischen Mächte Beachtung fand. Aufgrund hoher Schulden im Zuge der Kriegsführung drohten ihm militärische Auseinandersetzungen insbesondere mit den USA und Tonga. ${ }^{31}$ Um den Konsequenzen zu entfliehen und die Macht der Kubuna-Konföderation innerhalb Fidschis zu erhalten, suchte er nach Schutz bei den Briten, indem er diesen zweimal anbot, Fidschi als Kolonie ins britische Königreich aufzunehmen. Beim zweiten Angebot 1874 willigten die Briten ein, da die staatsbildenden Kriege abebbten, ein Bedarf nach Ordnung und Sicherheit für die wachsende Zahl der europäischen Siedler beanstandet wurde und eine stabile (Kolonial-)Regierung wirtschaftliches Potential versprach (Burns 1963: 90-96).

\subsubsection{Anfänge der kolonialen Ära - Landrecht und indische Kontraktarbeiter}

Um Fidschi als Kolonie aus Sicht der Briten möglichst gewinnbringend zu betreiben, benötigte es eines effizienten Verwaltungsapparates. Einerseits wollte man die eigene Machtposition sichern und ausbauen, um durchsetzungsfähig zu sein, andererseits sollten die Herrschaftsstrukturen kostengünstig und damit schlank gestaltet werden. Die Fidschianer wollte man daher weitestgehend einer Selbstverwaltung überlassen, mit der ein hoher Grad an Autonomie einhergehen sollte. Zudem fühlte sich die Kolonialregierung einer Bewährung fidschianischer Kultur verpflichtet (Jolly 1992: 330f.) So wurden Regierungs- und Verwaltungsstrukturen geformt, die sich an den drei östlichen Konföderationen anlehnten.

Ratu Cakobau dankte 1874 als Tui Viti ab und Königin Victoria sowie die ihr nachfolgenden britischen Monarchen beerbten den Titel. Bereits 1876 wurde der

\footnotetext{
${ }^{31}$ Das heutige Tonga kann als eine vierte Konföderation gesehen werden, die in der ersten Hälfte des 19. Jahr-hunderts unter Siaosi Taufa' ahau Tupou vereint wurde und sich unter seinem Nachfolger Enele Ma'afuotu'itoga, in Fidschi schlicht als Ma'afu bekannt, in kriegerischen Auseinandersetzungen mit den anderen Konföderationen befand (Burns 1963: 72-74, 78). Auch aktuell werden noch offizielle Forderungen geäußert, die Lau-Gruppe aufgrund der gemeinsamen Vergangenheit in Tonga einzugliedern (Gopal 2014).
} 
Große Häuptlingsrat (Great Council of Chiefs, GCC) geschaffen, ein die Kolonialregierung beratendes Gremium, das sich aus Häuptlingen der drei östlichen Konföderationen zusammensetzt. Es sollte die gesamte koloniale Ära überdauern und wurde auch im unabhängigen Fidschi beibehalten, ehe es 2012 aufgelöst wurde (Tuiwavu 2012). Insbesondere zu Bestimmungen, die die Lebensweisen der Indigenen beeinflussen, sollte der GCC befragt werden und dieser entsandte Repräsentanten für indigene Belange ins koloniale Parlament. An der Festschreibung des Landrechts zeigt sich besonders anschaulich die Verzahnung britischer Rechtstraditionen mit den Vorstellungen des GCC.

Marktwirtschaftliche Betrachtungsweisen von Land als ökonomisch nutzbarer Ressource, welche man besitzen könne, finden keine Entsprechung in traditionellen fidschianischen Denkweisen. Land, vanua, stellt demnach keine nichtmenschliche Umwelt dar, sondern ist integraler Bestandteil der Gesellschaft. Im vanua wirken die Ahnen. Deren mana, eine göttliche Kraft, macht die Umgebung fruchtbar und wohlwollend für ihre Nachfahren. Verwandtschaftsgruppen fühlen sich bestimmten Gebieten zugehörig. Die Zugehörigkeiten der Erblinien und die Gebietsausdehnungen unterliegen dynamischen Aushandlungsprozessen. Land wurde daher nie ausschließlich in seiner physischen Dimension gedacht, sondern ist Kulturlandschaft par excellence, mit sozialen und spirituellen Dimensionen versehen (siehe Kap. 7).

Die fidschianische Gesellschaft gliedert sich oberhalb der Haushalts- bzw. Familienebene in folgende soziale Einheiten: tokatoka (Großfamilie), mataqali (Klan), yavusa (Stamm), vanua (Stammeszusammenschluss) (Ravuvu 1987: 16). Im Rahmen dieses Häuptlingswesens wurde der Zugang zu Land für die Gemeindemitglieder geregelt. Zur kolonialen Ressourcenausbeutung waren diese Vorstellungen untauglich, über klare Besitzverhältnisse wollte man die Plantagenwirtschaft antreiben. Landbesitz wurde der mataqali-Ebene zugeschrieben, sodass Land nun als Gemeinschaftsbesitz ins britische Rechtverständnis aufgenommen wurde. Im Sinne lokaler Selbstverwaltung konnten die Gemeinden individuelle Zugänge zu Land jedoch selbst ausgestalten. Die Kolonialregierung schuf damit einen Rechtspluralismus (Benda Beckmann 2005: 3f.), in dem staatliche Landgesetze auf traditionelle Zugangsrechte stießen.

Ländereien, die vor dem Zeitpunkt der Festschreibung 1876 von weißen Siedlern bereits als Plantage genutzt wurden oder ihnen vertraglich von Fidschianern zugesichert wurden, wurden hingegen als Individualbesitz gewertet. Gebiete, auf die keine Ansprüche gemeldet wurden, sowie Gebiete um die aufkommenden Städte, wie die spätere Hauptstadt Suva im Südosten Viti Levus oder Levuka auf Ovalau, verfielen der britischen Krone. Das kommunale Land der Fidschianer durfte nicht verkauft, sondern lediglich verpachtet werden. ${ }^{32}$ Insgesamt verblieben nach dieser bis heute andauernden Regelung jedoch über $80 \%$ des Landes als

32 Lediglich zwischen 1905 und 1909 kam es zu einer Aufweichung des Verbots Kommunalbesitz zu verkaufen und ca. 8.000ha gingen in Individualbesitz über. 
Gemeinschaftsbesitz den fidschianischen Klans (Burns 1963: 107, 137-39; Lawson 2004: 525f., Weber 2007: 215). Die Pachteinnahmen verteilten sich nach einem Schlüssel nicht egalitär auf die Mitglieder des mataqali, neben Verwaltungskosten gingen gesonderte Beträge an die Häuptlinge, nicht nur auf mataqali-Ebene, sondern auch an die Oberhäupter des yavusa und vanua. Ein solcher Verteilungsschlüssel für Pachteinnahmen bestand bis in die jüngste Vergangenheit Fidschis; 2004 sah der Schlüssel noch vor, dass 15\% auf Verwaltungskosten und 30\% auf die Häuptlinge entfielen (Weber 2007: 215). Dieser Verteilungsschlüssel diente entscheidend als Einnahmequelle dem Machterhalt der Häuptlingsfamilien, die diese Regelungen im GCC ab 1876 mitbestimmten.

Ende des 19. Jahrhunderts etablierte sich Zuckerrohr zum wichtigsten Anbauprodukt der Plantagenwirtschaft. Aufgrund verschiedener Epidemien, die von den Kontakten mit den weißen Siedlern ausgelöst wurden, verstarb im 19. Jahrhundert mindestens ein Viertel der fidschianischen Bevölkerung, sodass nach dem Ausbruch der Masern 1875 die Population Ende des Jahrhunderts auf einem historischen Tiefpunkt stand (Burns 1963: 100f.; Gravelle 1988: 135-39). Neben dem Problem der geringen Bevölkerungsdichte stellte eine weit verbreitete Auffassung einen Ungunstfaktor für die Plantagenwirtschaft dar, welche besagt, dass Fidschianer - gemäß dem Zeitgeist entsprechender, rassistisch-diskriminierender, teilweise geodeterministisch aufgeladener, imperialistischer Theorien - weder willig noch fähig seien, auf Plantagen produktiv zu arbeiten (Burns 1963: 82; Gördes 1916: 17-20, 28-31; Graeffe 1868: 18f.). Auch aufgrund der Epidemien sollten die Fidschianer in ihren Dörfern verbleiben, wenig reisen und daher keiner Lohnarbeit auf Plantagen nachgehen (Gravelle 1988: 147). Als Alternative warb man für Kontraktarbeiter, zwischenzeitlich aus umliegenden Inselnationen, vor allem aber aus Indien. Zwischen 1879 und 1916 wurden über 60.000 indische Kontraktarbeiter zunächst für fünf oder zehn Jahre übergesiedelt, von denen die meisten nach Ablauf des Vertrages blieben. Nach Ablauf der Kontraktarbeit pachteten viele Land bei den Fidschianern, da sie im bestehenden Landrechtssystem kaum Chancen auf individuellen Landbesitz hatten. Mitte des 20. Jahrhunderts stellten IndoFidschianer aufgrund höherer Geburtenraten die Bevölkerungsmehrheit in Fidschi (Burns 1963: 110-12; Weber 2007: 209f.).

Die einheimischen Fidschianer nennen sich selbst bezeichnenderweise „iTaukel", eine Kurzform von „Taukei ni qele“, was mit „Besitzer des Bodens“" bzw. „Landbesitzer“ übersetzt werden könnte (Burns 1963: 1; Ravuvu 1983; Scarr 1984: ix). ${ }^{33}$ Dies zeigt die Bedeutung des Landtitels für das Selbstverständnis. Auch wenn die Festschreibung des Landrechts als Gemeinschaftsgut auf mataqaliEbene die ehemaligen, flexiblen und an sich verändernde soziale Konstellationen

\footnotetext{
${ }^{33}$ Ein Wort für „Fidschianer“ existiert im Fidschianischen nicht. In Tonga wurde James Cook von Feejee berichtet, woraus sich das englische Fiji ableitet. Namensgebend ist die Hauptinsel „Viti Levu“. Ich benutze die im Deutschen gängigste Schreibweise. Alle Staatsangehörigen werden als Fidschianer bezeichnet. Sofern eine Unterscheidung nötig ist, werden die indisch-stämmigen Fidschianer, als Indo-Fidschianer ausgewiesen und die Einheimischen als iTankei oder autochthone Fidschianer.
} 
anpassbaren Glaubensvorstellungen von vanua (Williams 1858: 15f.) massiv beeinträchtigte, konnte man sich im Laufe der Generationen nicht nur mit den Konzepten von (gemeinschaftlichem) Landbesitz arrangieren, sondern diese gar zu einer Quelle der eigenen kulturellen Identität machen. Dies bedeutet jedoch nicht, dass individueller Zugang zu Land innerhalb der Gemeinden konfliktfrei geregelt sei (siehe Kap. 7.3.4). Weiterhin kann iTaukei zur Abgrenzung von den vulagi, Gästen, zu denen dieser Sprachkonstellation entsprechend die Indo-Fidschianer zählen, politisch missbraucht werden (siehe Kap. 5.2.1).

\subsubsection{Ethnische Spaltungen im Zuge der Kolonialpolitik}

Die Politik der Kolonialregierung sorgte für ein Nebeneinander der beiden Ethnien. Interaktionen waren gering. Die Indo-Fidschianer, die auf Zuckerrohrfeldern arbeiteten, lebten in eigenen Siedlungen. Viele Indo-Fidschianer zogen zudem in die Städte, um dort einer Lohnarbeit nachzugehen. So konnte sich eine kleine wirtschaftliche Elite unter den Indo-Fidschianern herausbilden. Bis in die 1960er waren die $i$ Taukei hingegen reisebeschränkt und blieben weitestgehend in ihrem dörflichen Umfeld; eine Gesetzgebung, die ihren einstigen Sinn in der Bekämpfung der Epidemien des vorherigen Jahrhunderts hatte. Politische Ambitionen, Integration und kulturellen Austausch zu fördern, waren gering. ${ }^{34}$

Die Rahmenbedingungen der Unterbringung auf überfüllten Schiffen während der Überfahrt und großer Arbeitskasernen zur Plantagenarbeit haben dazu geführt, dass das Kastenwesen in Fidschi nicht aufrecht erhalten blieb. Mangelnder Kontakt zum Heimatland aufgrund der geographischen Distanzen beförderte im Laufe der Jahrzehnte eine eigenständige Weiterentwicklung zu einer indofidschianischen Kultur (Burns 1963: 111f.; Voigt-Graf 2008: 84-91). Aufgrund oftmals desaströser Arbeitsbedingungen, unter denen die Indo-Fidschianer auf den Plantagen arbeiten mussten, organisierten diese sich bereits Anfang des 20. Jahrhunderts gewerkschaftlich bzw. parteipolitisch. Auch die Vorauswahl der Kontraktarbeiter, diese aus möglichst diversen sprachlich-kulturellen Regionen Indiens und unterschiedlichsten Kasten herzulocken, um gemeinsame Zusammenschließungen zu erschweren, konnte einen organisierten Widerstand in Form von Streiks nicht unterbinden. Aufgrund des hohen Grades politischer Organisation und der noch bestehenden kulturellen Nähe zu den Unabhängigkeitsbewegungen in Indien um Gandhi kamen erste Unabhängigkeitsbestrebungen vor allem aus dem Indo-Fidschianischen Lager. In den 1930ern wurden dabei Vorstellungen eines unabhängigen indischen Staates mit ethno-fidschianischer Minderheit, oder aber die Eingliederung in eine Indische Union geäußert (Scarr 1984: 149).

Diese Träume waren jedoch fernab jeglicher Realität, da die indofidschianische Bevölkerung anfänglich gar nicht und später äußerst unterrepräsentiert an der Politik des Kolonialstaates beteiligt war. Insbesondere auf nationaler

${ }^{34}$ Eine Ausnahme wäre beispielsweise das seit 1956 bestehende, jährliche Hibiscus Festival zur Förderung eines interkulturellen Austauschs (Burns 1963: 8f.) 
Ebene fand politisches Denken und Handeln in ethnischen Blöcken statt. Der Legislativrat, kolonialer Vorläufer eines Parlaments, setzte sich zusammen aus nominierten und gewählten Vertretern nach regionalem und ethnischem Proporz. Anfänglich mussten sämtliche gewählten und der Großteil der vom Exekutivrat bestimmten Mitglieder eine europäische Herkunft nachweisen. Zwei nominierte Angehörige des Legislativrats mussten autochthon sein und erst ab 1916 wurde ein Vertreter der indisch-stämmigen Gemeinde nominiert. Ab 1929 wurde jener Repräsentant von den Indo-Fidschianern gewählt (Burns 1963: 126f.). Die Spaltung der Politik nach ethnischen Merkmalen zur Kolonialzeit beförderte das Nebeneinander der Ethnien anstelle einer Politik des Gesamtwohls des Nationalvolkes $^{35}$ und eines transkulturellen Zusammenlebens.

Belange der autochthonen Fidschianer regelte zusätzlich ein Department für Einheimische Angelegenheiten (Native Affairs Department, später umstrukturiert als Fijian Administration), welches ihnen eine privilegierte Stellung sichern und ihre kulturellen Werte bewahren sollte. In den 1910er Jahren wurden hierzu bereits vereinzelt kritische Meinungen geäußert, die politische Doppelstruktur zur Festschreibung der fidschianischen Kultur verhindere Möglichkeiten einer Veränderung und Entwicklung (Burns 1963: 132-34). Ein Beispiel für die Schäden die ein konservatives Verharren auf vermeintlich kulturelle Werte verursachen kann, sind die Reisebeschränkungen, die einst u.a. zur Bekämpfung von Epidemien ins Leben gerufen wurden. Sie blieben bestehen, als sie schon lange nicht mehr zeitgemäß waren, um nunmehr uminterpretiert die „dörfliche“ Kultur Fidschis zu wahren und erschwerten Chancen auf institutionalisierte Bildung und ökonomischen Aufstieg - zweier (nunmehr) hochgeschätzter sozio-kultureller Werte. Dies zeigt idealtypisch das Spannungsfeld gesellschaftlicher Transformation in dem sich die Fidschianer seit der Einflussnahme durch die Europäer befinden. Weder ein blindes, zügiges Assimilieren an die europäischen Kulturen, noch ein Versteifen auf angeblich ureigene kulturelle Werte kann dauerhaft Lebensqualität erzeugen. Stattdessen muss Tradition als ein Prozess verstanden werden, der über die Zeit Veränderungen in Wertvorstellungen und Anpassungen an sich verändernde gesellschaftliche Umstände zulässt. Der hybride Charakter der Kultur als Mischung vorkolonialer und kolonialer Praktiken, Werte und Glaubensvorstellungen muss dann nicht als verwässert interpretiert und als Hemmnis der Lebensqualität gedeutet werden. Stattdessen weist die einzigartige Durchmischung eine Eigenständigkeit und Flexibilität auf, aus der eine Anpassungsfähigkeit als Aktivum zur Erhöhung der Lebensqualität generiert werden könnte. So könnte die Wahrung „dörflicher" Werte vereinbar mit Bildungschancen und ökonomischem Erfolg werden.

\footnotetext{
${ }^{35}$ Im deutschen Sprachgebrauch existiert leider keine Unterscheidung des Volksbegriffes nach politischen oder ethnischen Gesichtspunkten. Im antiken Griechenland hingegen wurde schon zwischen demos und ethnos unterschieden. Ich verwende hier den Begriff des Nationalvolkes um deutlich zu machen, dass es aus politischer Perspektive sehr wohl ein „Volk“ geben kann, welches sich nicht nach ethnischen Merkmalen definiert.
} 
Im Zuge des politischen Aufbegehrens der Indo-Fidschianer verweigerte diese Bevölkerungsgruppe nahezu kollektiv den Militärdienst im Zuge der Weltkriege (Gravelle 1988: 188-92), sodass sich das Militär bis heute überwiegend aus iTaukei zusammensetzt. ${ }^{36}$ Im Zweiten Weltkrieg dienten über 11.000 Männer den fidschianischen Streikkräften. Im August 1943 waren zeitgleich 8.513 Menschen beim Militär, davon 6.371 iTaukei - was rund 12\% der männlichen Bevölkerung entsprach (Scarr 1984: 145) - gegenüber 264 Indo-Fidschianern (Burns 1963: 146). Während Ratu Sir Lala Sukuna - als Kriegsheld des ersten Weltkriegs, OxfordAbsolvent, hoher Häuptling und später erster fidschianischer Vorsitzender des Legislativrates der Kolonialregierung einer der größten Staatsmänner Fidschis den Freiwilligendienst erfolgreich bewarb, um Fidschi internationale Anerkennung zu verschaffen, verdeutlichten die Zahlen der Indo-Fidschianer die ethnische Spaltung und schürten Ressentiments gegen diese Bevölkerungsgruppe. In politischer Rhetorik der Nachkriegszeit seitens vieler iTaukei wurde die knappe Bevölkerungsmehrheit, die die Indo-Fidschianer seit der Volkszählung 1946 innehatten, als „Indischer Kollossus“ dargestellt, welcher nach alleinigem Machtanspruch greife, um die mehrheitlichen Bestrebungen nach Gleichberechtigung zu diffamieren (Scarr 1984: 158f.).

Ein Wunsch nach Reformen, mehr Mitbestimmung und Verantwortung, sowie letztlich eine Unabhängigkeit von Großbritannien ging vor allem von den indofidschianischen politischen Organisationen aus und zeigte sich öffentlich vor allem in Streiks. Die $i$ Taukei hingegen wurden apolitisiert bzw. fühlten sich über das Häuptlingswesen politisch repräsentiert und wurden kaum parteipolitisch aktiv. Der GCC stand einer Unabhängigkeit mitunter skeptisch gegenüber, da die Mitglieder Einschränkungen ihres eigenen Einflussvermögens befürchteten. Begleitend von einer dem kolonialem Imperialismus kritisch gegenüberstehenden, an Einfluss gewinnenden, internationalen Öffentlichkeit, setzten sich ab den 1960er Jahren Reformbewegungen durch. Diese führten schrittweise zu mehr demokratischer Selbstverwaltung der Kolonie, einem Frauenwahlrecht und mehr Einflussmöglichkeiten der indisch-stämmigen Fidschianer und mündeten in die Unabhängigkeit am 10. Oktober 1970.

\subsection{Gesellschaftliche Spannungsfelder und politische Konfliktlinien seit der Unabhängigkeit}

Die Kolonialzeit hat die fidschianische Kultur zutiefst beeinflusst. Auch die kulturpolitische Ausrichtung der Kolonialregierung, die Kultur Fidschis bewahren zu wollen, änderte nichts daran. Im Gegenteil stellte der Versuch einer Bewahrung des Traditionellen eine extreme Form der Intervention dar. Die Forcierung eines

\footnotetext{
36 Zur Volkszählung im Jahre 2007 wurden 4359 Menschen auf Fidschi als Streitkräfte registriert. Von ihnen waren 4325 iTaukei gegenüber 28 Indo-Fidschianern. 86 Armeeangehörige waren Frauen (FIBOS 2012: 105). Fidschianische Soldaten, die zum Erhebungszeitpunkt in Übersee stationiert waren, fallen vermutlich aus dieser Statistik.
} 
Stillstandes als ethnisch homogene Dorfkultur behindert Anpassungsprozesse Die kulturelle Sphäre einer Gesellschaft kann nicht losgelöst von den sonstigen gesellschaftlichen Dimensionen betrachtet werden. Politischer und ökonomischer Wandel, sowie Veränderung sozialer und religiöser Praktiken, welche die Kolonialzeit zweifelsohne mit sich brachten, wirkten sich zwangsläufig auf die Kultur aus. Kultur zu depolitisieren und wie unter einer Haube konservieren zu wollen, ist ein hochgradig politischer wie kulturbeeinflussender Akt. Eine Kulturpolitik der Stagnation zu betreiben, während die Gesellschaft einen tiefgreifenden Wandel unterläuft, ist aufgrund des prozesshaften Charakters von Kultur mittel- bis langfristig ein hoffnungsloses Unterfangen. Hinsichtlich einer Konfliktlösung ist sie kontraproduktiv. Sie verstärkt gesellschaftsinhärente Spannungen, die ein Transformationsprozess ohnehin mit sich bringt, da nötige Anpassungen an ein sich veränderndes sozio-kulturelles Umfeld gestaut werden. Kulturpolitischer Traditionalismus befördert eine Ungleichzeitigkeit gesellschaftlicher Entwicklungen. Im unabhängigen Fidschi entladen sich die verschleppten Konflikte zum Teil explosionshaft.

\subsubsection{Ethnische Konflik.te in Fidschi}

Im Laufe der kolonialen Ära hat sich die Zusammensetzung der fidschianischen Bevölkerung nach ethnischen Merkmalen erheblich verändert. Es kam zur Zuwanderung von Europäern, welche Schlüsselpositionen in Wirtschaft und Politik bekleideten. Hinsichtlich eines Konfliktpotentials bedeutsamer ist jedoch, dass die Nachfahren der indischen Kontraktarbeiter zum Zeitpunkt der Unabhängigkeit die größte Bevölkerungsgruppe stellten und dies mittels politischer Rhetorik seitens vieler $i$ Taukei als Bedrohung wahrgenommen wurde und wird.

Befördert von der Kulturpolitik der Kolonialregierung genossen die ethnischen Fidschianer Rechte und Privilegien, auf die viele bis heute Ansprüche melden und diese nicht veräußern wollen. Dies betrifft insbesondere das Landrecht, sowie den GCC als Vertretung einheimischer Interessen, welcher im Zuge der Unabhängigkeit mit einem Vetorecht bezüglich politischer Entscheidungen, die die fidschianische Kultur berühren, ausgestattet wurde. In der Verfassung wurde verankert, dass das Landrecht nur bei einer zwei Drittel Mehrheit im Parlament sowie einer dreiviertel Mehrheit im Großen Häuptlingsrat geändert werden kann (Lal 2003: 344; Weber 2007: 213). Da die Mitglieder des Häuptlingsrats ökonomisch gesehen aufgrund des Verteilungsschlüssels der Pachteinnahmen persönlich die größten Nutznießer des bestehenden Landrechts sind, sind Veränderungen nahezu ausgeschlossen.

Das Wahlsystem im unabhängigen Fidschi behielt den Zwang regionaler wie ethnischer Proporze im Parlament bei. Die Parteien stellten sich im Wesentlichen nach ethnischen Merkmalen auf und betrieben Klientelpolitik. Aufgrund der Unterstützung der europäisch-stämmigen Bevölkerung gewann die ethnofidschianische, national-konservative Alliance Party um Ratu Sir Kamisese Mara 
die ersten Wahlen im unabhängigen Fidschi. Ratu Mara war drei Legislaturperioden lang bis 1987 Premierminister, ehe er von Timoci Bavadra abgelöst wurde, welcher mit der vorwiegend indo-fidschianisch ausgerichteten Labour Party die Wahlen gewann. Er konnte sich jedoch nur einen Monat im Amt behaupten. Mittels zweier unblutiger Militärputsche hievte der hochrangige Militär Sitiveni Rabuka seinen Häuptling Ratu Mara zurück ins Amt. Öffentlich gerechtfertigt wurden die Coups mit der Wahrung fidschianischer Interessen. Die politische Rhetorik der Wahl-Verlierer skizzierte einen Angriff auf den „Fïjan Way of Life" (vaka i tanker).

Der vaka i taukei stelle - so die ethnokulturelle Propaganda - eine Symbiose von Entwicklung und Moderne mit der Aufrechterhaltung kultureller Werte und Traditionen dar. Er fordert damit Wachsamkeit gegenüber importierten, kulturellen Praktiken, die in diesem Sinne eine Degradation der hochwertigen eigenen Tradition, Seele und des kulturellen Erbes darstellen (Mückler 1998: 411-12, Ravuvu 1983). iTaukei sollen sich demzufolge als Haus- und Landbesitzer und Gastgeber interpretieren. Normativ soll ihr Verhalten von Höflichkeit, Respekt und Gastfreundschaft geprägt sein gegenüber den Indo-Fidschiandern, welche als vulagi, Gäste, dargestellt werden. Diese unterliegen normativ ebenfalls Rechten und Pflichten gegenüber ihrer Gastgeber. Dieses dichotome, mitunter diskriminierende Verständnis des eigenen Nationalvolkes als in zwei Klassen gespaltet ist tief in das Selbstverständnis der autochthonen Fidschianer eingegangen (Newland 2007: 304-306).

Auch wenn Timoci Bavadra iTaukei ist, so wurde der Wahlerfolg 1987 der Labour Party als Attacke gegen die „legitime“ Herrschaftsordnung zwischen Gästen und Gastgebern dargestellt. Die Absetzung Ratu Maras durch die vulagi sei demzufolge eine Anmaßung. ${ }^{37}$ Der Regierungssturz durch das Militär zum Zwecke der Reinstallation Ratu Maras wurde von weiten Teilen der Bevölkerung toleriert und in den Städten kam es zu Übergriffen und Plünderungen indo-fidschianischer Geschäfte. Von einer Verfolgung der Straftäter wurde abgesehen. Viele IndoFidschianer verließen daraufhin fluchtartig das Land und seither gibt es eine konstant hohe Abwanderungsrate seitens der Indo-Fidschianer. Zwischen den Jahren der Volkszählung 1986 und 2007 verließen über 80.000 Indo-Fidschianer das Land und ihr Bevölkerungsanteil sank von 48,7\% auf 37,5\%. Da vor allem die produktivsten Menschen auswandern, die andernorts verhältnismäßig gute Chancen auf dem Arbeitsmarkt haben, schlägt er sich im Mangel an qualifizierten Arbeitskräften, vor allem im Ausbildungs- und Gesundheitssektor des Landes nieder (FIBOS 2008: 3, 17; Lal 2003: 346, Reddy et al. 2004: 1450-53). Aufgrund ihrer pazifischen Identität kehren die meisten nicht etwa nach Indien „zurück“, sondern

\footnotetext{
${ }^{37}$ Als eine weitere Konfliktdimension kommt erschwerend hinzu, dass nach traditionellem Werteverständnis im Häuptlingssystem Oberhäupter zwar über Wahlen oder sonstige, demokratieähnliche Verfahren auf ihre Position kommen können (siehe Kap. 6); eine Absetzung ist jedoch nicht vorgesehen und würde eine Beleidigung darstellen. Die Abwahl eines Staatsoberhauptes verletzt demnach kulturelle Werte.
} 
wandern in die wirtschaftsstarken pazifischen Anrainerstaaten Australien, Neuseeland, Kanada oder in die USA aus (Voigt-Graf 2008: 83, 95f.). Zudem ist das natürliche Bevölkerungswachstum der Indo-Fidschianer seit dem ersten Putsch drastisch gesunken, was nicht nur auf den sich selbst verstärkenden Rückkopplungseffekt eines selektiven Fortzug von Menschen im reproduktionsfähigen Alter, zurückzuführen ist. Auch die Fertilitätsrate ist zwischen 1986 und 2007 von 2,7 auf 1,7 Kinder pro Frau gesunken (Lewai 2010: 86), also unter dem Reproduktionsniveau, was ein (zeitverzögertes) Schrumpfen der Bevölkerung bedeutet; während die Fertilitätsrate der $i$ Tankei hingegen bei über 3 Kindern pro Frau verblieb.

Trotz Verfassungsänderungen, die die Rechte des GCC stärkten und die festgeschriebene Mehrheit von iTaukei im Parlament ausbauten, gewann 1999 überraschend die Labour Party die Wahlen und der Indo-Fidschianer Mahendra Chaundry wurde Premierminister. Nach nur einem Jahr im Amt wurde durch den Zivilisten George Speight ein dritter Putschversuch unternommen. Unterstützt von einer Eliteeinheit des Militärs stürmte er das Parlament und hielt die Regierungsbeamten 52 Tage lang als Geiseln. Auch wenn hier persönliche Motive wegen Differenzen zwischen Speight und Chaundry offenkundig waren (Weber 2007: 211), versuchte er den Putsch mit der Wahrung fidschianischer Interessen zu rechtfertigen. Der Großteil der Streitkräfte stellte sich jedoch hinter ihren obersten Militärkommandeur, Ratu Josaia Voreqe Bainimarama und damit gegen die Putschisten. Während der Zeit der Geiselnahme installierte Bainimarama eine Übergangsregierung unter Laisenia Qarase, einem Vertrauten des GCC. Dieser gründete eine ethno-fidschianische, konservative Partei, mit der er die Neuwahlen 2001 gewann. Für Speight schlug der Putsch zwar fehl, er und ein Großteil seiner Gefolgsleute wurden inhaftiert, im Sinne der Bewahrung des "Fijian Way of Life“ war er jedoch ein Erfolg. Details, wie Bainimarama Präsident Mara dazu verleitete, von der Verhängung des Kriegsrechts Gebrauch zu machen und wie er ihn vom Rücktritt als Präsident überzeugen konnte und so den Weg für eine militärische Übergangsregierung freigab, sind bis heute un-bekannt (Field 2007: 178; Weber 2007: 212). Manche sehen die Umstände als einen Putsch innerhalb eines Putsches (Watson 2005: 362).

In der Zeit um Speights Putschversuch kam es erneut zu Pogromen gegen die Indo-Fidschianer, woraufhin viele fluchtartig das Land verließen. Die neue Regierung unter Qarase heizte die Resentiments mitunter an, so plädierten sie in der Öffentlichkeit, auslaufende Pachtverträge mit Indo-Fidschianern nicht zu verlängern. Als Qarases national-konservative Partei die ordentlichen Wahlen 2006 gewann, putschte Bainimarama seinen ehemaligen Schützling aus dem Amt und installierte eine Militärdiktatur. Dieser Putsch wurde als Kampagne gegen rassistische Tendenzen und gegen Korruption begründet (Bendt 2006: 2-4; Ratuva 2007: 
27, 35-37)..$^{38}$ Obwohl dieser als pro-indo-fischianisch - oder zumindest als Versuch einer Annäherung und Normalisierung - angesehen werden kann, gehen offizielle Schreiben davon aus, dass auch seit der letzten Volkszählung 2007 eine Emigration seitens der Indo-Fidschianer weiterhin anhält (FIBOS 2012: 3). Der heute im Exil lebende Indo-Fidschianer und Historiker Brij Lal drückt die Dimensionen dieser Tragödie in folgenden, symbolträchtigen Zahlen aus: Einst kamen 60.000 Inder als Kontraktarbeiter nach Fidschi, seither haben 120.000 das Land wieder verlassen (Lal 2012).

\subsubsection{Konflikte entlang der Häuptlingskonföderationen}

Neben den ethnischen Konflikten, welche sich in der jüngeren Geschichte Fidschis mehrfach gewaltsam niederschlugen, schwelen unterschwellig weitere Konflikte. So existiert eine zweite Konfliktlinie, die innerfidschianisch entlang der drei Konföderationen Tovata, Kubuna und Burebasaga ausgetragen wird (Lawson 2004: 520). Ratu Mara war als Tui Lau und Tui Nayau oberster Häuptling der Tovata-Konföderation. Zugleich war er mit verheiratet mit Ro Litia Cakobau Lalabalavu Katoafutoga Tuisawau, dem Oberhaupt der BurebasagaKonföderation. Die Bedeutung solcher Heiratsallianzen auf das Machtgefüge zwischen den jeweiligen Konföderationen wird kontrovers diskutiert. Als Ratu Mara das Amt des Premierministers 1987 an Timoci Bavadra verlor, putschte in Person von Rabuka ein Adliger der Tovata-Konföderation seinen ParamountHäuptling zurück an die Macht. 1992 wurde Rabuka zum Premierminister gewählt und Ratu Mara übernahm das Amt des Präsidenten.

Von den drei großen Konföderationen fühlen sich in erster Linie die iTaukei der östlichen Inseln repräsentiert. Bavadra wurde in Lautoka, dem Westen Fidschis, geboren. Während im Westen sowohl durch den Tourismus als auch durch den Goldabbau und die Zuckerrohrwirtschaft das ökonomische Zentrum liegt, ist die Region politisch unterrepräsentiert. Es gibt daher Bestrebungen, eine vierte, westliche Yasayasa-Vaka-Ra-Konföderation zu errichten. George Speight hätte einen Häuptling der Burebasaga-Konföderation als Premierminister vorgesehen, sowie den Turaga Taukei Naua, einen Häuptling aus Nadi, dem Westen Fidschis, als Präsidenten. Die Staatsstreiche zielten somit auch auf Machtverschiebungen zwischen den Politischen Größen der iTaukei ab.

Mit den Verfassungsänderungen der 1990er Jahre sollte eine indofidschianische Machtbasis illusorisch werden. Nur weil sich die ethnofidschianisch aufgestellten Parteien aufgrund von Grabenkämpfen entlang ihrer Konföderationslinien selbst schwächten und viele iTankei die Labour Party wählten, konnte 1999 ein Indo-Fidschianer Premierminister werden. Während Bainimarama Mitglied der Kubuna-Konföderation ist, verhalf er zunächst im Zuge

\footnotetext{
${ }^{38}$ Eine gewisse Ironie birgt der Umstand, dass Bainimarama den Putsch zuvor mehrfach angedroht hatte, da er die Regierung als zu nachlässig ansah, Rechtsstaatlichkeit zu gewährleisten und künftige Putschversuche zu verhindern.
} 
der Geiselnahme seitens George Speight einem weiteren Häuptling der TovataKonföderation, Qarase, zum Premierminister. Ratu Mara dankte derweil als Präsident ab und dessen Vize Ratu Josefa Iloilovatu Uluivuda von der KubunaKonföderation wurde sein Nachfolger. Nach dem Coup 2006 war Bainimarama selbst Premierminister und auch als Ratu Epeli Nailatikau 2009 die Nachfolge als Präsident antrat, verblieben die beiden höchsten Staatsämter in der KubunaKonföderation.

Inwiefern die politischen Machtspiele zwischen und innerhalb der Konföderationen anstelle der ethnischen Konfliktdimensionen ausschlaggebend für die verschiedenen Putsche waren, kann nicht abschließend geklärt werden. Innerhalb der iTaukei identifiziert man sich stark mit dem Häuptlingssystem. Dennoch ist dieses nicht frei von Kritik und der TLTB-Verteilungsschlüssel von Pachteinnahmen sowie politische Positionierungen der großen Häuptlinge werden nicht vorbehaltslos hingenommen, wie insbesondere ein Wahlverhalten losgelöst von der Konföderationszugehörigkeit zeigt.

\section{Bevölkerung nach Religionen}

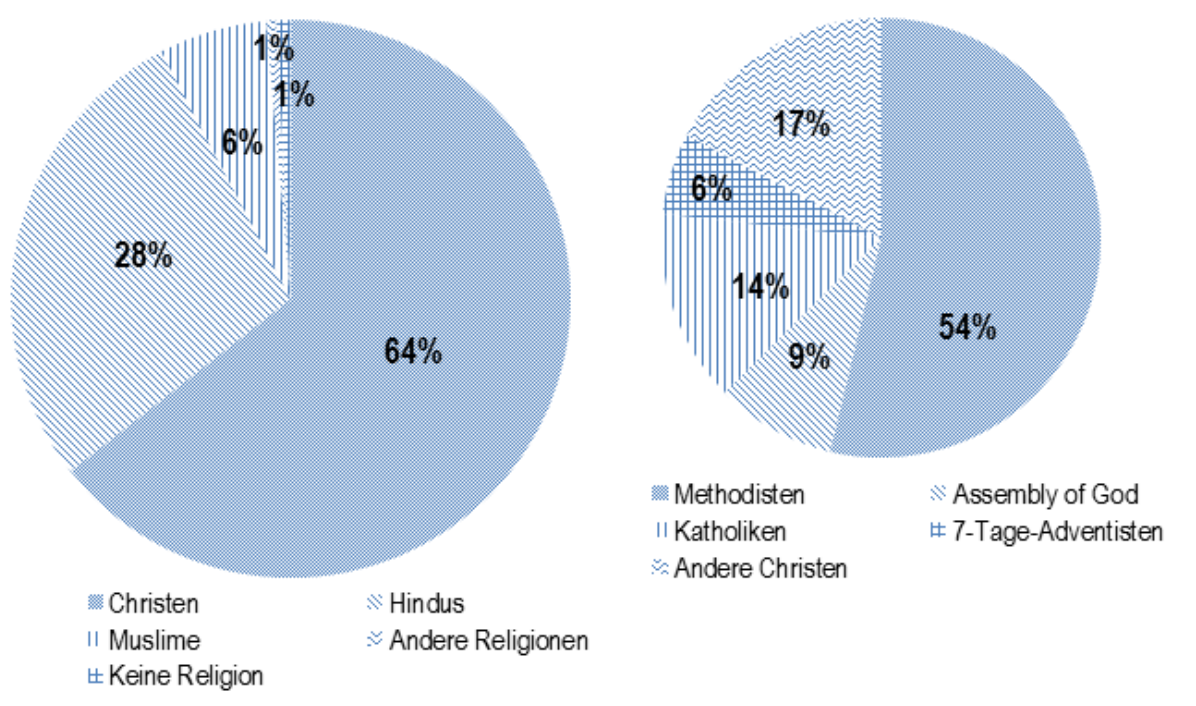

\section{Zusammensetzung Christen}

Abb. 2: Bevölkerungszusammensetzung binsichtlich Religionen - Stand 2007 (Eigene Darstellung nach FIBOS 2012: 10)

Weiterhin könnten Interessen ausländischer Akteure die politische Instabilität Fidschis entscheidend verursacht haben. So setzte sich das Opfer des ersten Putsches, Timoci Bavadra, aktiv gegen Atomwaffenversuche im Pazifik ein und stand damit vor allem den Interessen der USA entgegen, während Ratu Mara 1983 die fidschianischen Hoheitsgewässer als atomwaffenfreie Zone aufgehoben hatte. 
Zudem wollte Bavadra die Goldmine verstaatlichen, welche in australischem Besitz war und seit der Unabhängigkeit Fidschis 1970 bis 1986 mit ca. 1Mio FJ-\$ nur etwa ein halbes Prozent ihrer Exporterlöse als Steuern abgab (Weber 2007: 21113), sodass die australische Wirtschaft vom ersten Putsch profitierte.

Weitere Konfliktdimensionen existieren im religiösen Bereich. Auch wenn auf Fidschi Menschen der drei Weltreligionen Christentum, Hinduismus und Islam zusammenleben (siehe Abb. 2), so geschieht dies weitestgehend friedlich. Die Interaktionen sind jedoch begrenzt, auch wenn einzelne religiöse Feste von den jeweils anderen Glaubensanhängern gerne mitg-feiert werden. Bestrebungen einzelner christlicher Strömungen, das Christentum zur Staatsreligion zu erheben (Newland 2009: 188; Weir 2015: 167, 172-74), verliefen bisher im Sande und konnten das friedliche Nebeneinander nicht nachhaltig stören. Lediglich bei Eheschließungen zwischen Menschen unterschiedlicher Religionen werden mitunter Ressentiments deutlich. Bedeutender sind hingegen religiöse Spannungen innerhalb der verschiedenen christlichen Glaubensrichtungen, welche eng mit den Häuptlingskonflikten verbunden sind.

Die $i$ Taukei sind fast ausschließlich Christen, über die Hälfte von ihnen gehören der methodistischen Denominierung an. Während sich die katholische Kirche ebenfalls frühzeitig etablierte, gewinnen seit mehreren Jahrzehnten Freikirchen an Einfluss. Die methodistische Kirche verstand es von Anfang an, sich im fidschianischen Häuptlingswesen zu bewegen. Sie deklarieren Häuptlinge als göttlich legitimiert, festigen deren Position in der Gesellschaft und werden dafür umgekehrt in ihren Anliegen gefördert. Bis heute arbeiten die obersten Häuptlinge aller Konföderationen eng mit den Oberhäuptern der Methodisten zusammen, üben zum Teil Doppelfunktionen aus (siehe Kap. 6.3.3), und unterstützen sich gegenseitig, sodass die methodistische Kirche starken politischen Einfluss ausüben kann und dies auch tut. Dabei vertritt sie insbesondere die Interessen ihrer Klientel, national-konservative iTaukei (Newland 2007: 302f., 308f.; Newland 2009: 188-190; Trnka 2011: 73f.).

\subsubsection{Konfliktfelder und Lösungsansätze in der Militärdiktatur}

Die gesellschaftspolitischen Konflikte auf den Fidschi-Inseln sind sehr vielschichtig und komplex miteinander verwoben. Es existieren nicht nur Spannungen zwischen Ethnien und Religionen, sondern vor allem innerhalb der jeweiligen Entitäten. Viele Ursachen der heutigen Problemdimensionen liegen in der kolonialen Vergangenheit, doch auch seit der formalen Unabhängigkeit ist der äußere Einfluss enorm. Der Putsch 2006 unter Bainimarama hatte offiziell das Anliegen, endgültig ,aufzuräumen“ und einen Schlussstrich unter die hemmende, rassistische Klientelpolitik und Putsch-Kultur zu ziehen (Ratuva 2013: 171). Er wurde 
ausgerufen als "coup to end all coups"39 (Fraenkel \& Firth 2009a: 4, 8; Fraenkel \& Firth 2009b: 455).

Eine Beurteilung der Militärregierung unter Bainimarama seit 2006 ist vor diesem Hintergrund schwierig. Man kann Bainimarama einfach als machthungrigen Diktator hinstellen. Sein anfängliches Auftreten als Militärgeneral verstärkte das Bild eines keinen Widerspruch duldenden Hardliners. Die Machtkonzentration auf seine Person zeigt sich daran, dass er neben seiner Funktion als Premierminister zusätzlich zeitgleich sechs von zwanzig Ministerien leitete (Wise 2014). Hinzu kommen Wortbrüche, Neuwahlen nicht 2009 sondern erst 2014 ausrichten zu wollen sowie das Verhängen einer Pressezensur (Davis 2009). Gerade letzteres lässt eine freie Meinungsbildung kaum zu und erschwert eine kritische Diskussion der politischen Ziele und Leistungen der Militärregierung. Ich werde hier einige kontrovers aufgefasste Aspekte aufgreifen, um sein ambivalentes Bild in der Bevölkerung zu vermitteln.

Nach einer anderen Lesart ist das Militär Fidschis weltweit im Kampf gegen scheiternde Staaten aktiv und hoch angesehen (Prasad 2009: 59), sodass Bainimarama sich als eigentlicher Verfechter von Rechtsstaatlichkeit und Ordnung im eigenen Lande zum Eingreifen genötigt sah (Bainimarama 2007: 1f.). Da Fidschi jedoch nicht den Kriterien eines fragilen Staates entspricht (Morcos \& Röder 2007: 290f.), ist der militärische Einsatz im eigenen Land nicht mit dieser Begründung zu entschuldigen. Die gestürzte Regierung ist jedoch einem Wahlrecht entsprungen, welches nach westlichem Demokratieverständnis nicht dem Grundsatz des gleichen Stimmrechts entsprach und sie hätte wohl eine Politik betrieben, die eine Eskalation der ethnischen Konfliktlinie angeheizt hätte. Die gestürzte Regierung entsprach damit ebenfalls keinem westlich-demokratischen Werteverständnis.

In der internationalen Politik reichten die Reaktionen von Reisewarnungen für deutsche Urlauber über das vorübergehende Aussetzen von Entwicklungsgeldern der USA bis hin zum Ausschluss aus dem Commonwealth (Bendt 2006: 4). Diese Maßnahmen suchen nicht die Diplomatie sondern betreiben eine Isolation. Einer Ursachenforschung für die prekäre politische Situation auf Fidschi wird nicht nachgegangen, die Verweigerung eines Dialogs trägt stattdessen zur Instabilität des Landes bei. Auch die Handelsbeziehungen und der Tourismus brechen nach jedem Coup ein, was jedes Mal zu Einbrüchen einer ansonsten florierenden Wirtschaft führte (Wolf \& Waibel 2010: 49f.). Es sind insbesondere Wirtschaftssektoren betroffen, in denen überdurchschnittlich viele Frauen arbeiten, sodass vor allem diese unter den Folgen zu leiden haben (Kotoisuva 2010: 241f.)

\footnotetext{
${ }^{39} \mathrm{Im}$ Wortlaut erklärte Bainimarama in einer Rede vor der UN: ,,To remove this coup culture and to commit to democracy and the rule of law, policies which promote racial supremacy, and further the interests of economic and social elites, must be removed once and for all. Racism, elitism and disrespect for the law are undemocratic. They lead to hatred, violence, poverty and moral bankruptcy. We saw that in the years leading to World War TWO. We saw the genocide, the concentration camps, the rampant imperialism which resulted in turn, in the creation of the United Nations" (2007: 5).
} 
Inwiefern die politische Praxis der Militärdiktatur ein politischer Klientelismus ist, oder aber dem Gesamtwohl dient, liegt mitunter im Auge des Betrachters. So hat Bainimarama den GCC nach gut 130 Jahren Existenz aufgelöst. Mit der Abschaffung der zweiten Volkskammer zementiert er die Macht des Premierministers, also seine eigene. Er begründet sie jedoch folgendermaßen:

The Great Council of Chiefs is a product of our colonial past and Fiji must now focus on a future in which all Fijians are represented on the same basis. (...) Fiji's iTaukei heritage is a distinct and fundamental aspect of Fiji (and) this cannot be denied. However, as an institution, the Great Council of Chiefs perpetuated elitism and fed into the divisive politics which plagued our country. We must now look to our commonalities as citizens of the same nation, not to what separate us as individuals or groups. (Bainimarama zitiert nach Tuiwavu 2012)

Nimmt man das vorgetragene Anliegen ernst, befreite er Fidschi von einer kolonialen Altlast, die viele der aktuellen Konfliktdimensionen ursächlich mitbegründet hat. Die ökonomisch untermauerte Machtposition der privilegierten Paramount-Häuptlinge wurde weiterhin geschwächt, indem der Verteilungsschlüssel der Pachteinnahmen aufgelöst wurde. Abzüglich einer Verwaltungskostenpauschale an das TLTB werden die Einnahmen nunmehr paritätisch auf sämtliche Mitglieder eines mataqali aufgeteilt (Nasiko 2012). Zusammengenommen bilden beide Maßnahmen einen extremen Umbruch im Häuptlingssystem. Auch wenn die Richtung der Veränderung durchaus einen positiven Beitrag zum Gesamtwohl der fidschianischen Bevölkerung vermuten lässt, so sind sie in ihrer Totalität und Geschwindigkeit zu radikal, als dass die resultierenden gesellschaftlichen Veränderungen keine Unsicherheiten nach sich ziehen würden.

So beklagten viele autochthone Fidschianer in Einzelgesprächen die Anfälligkeit des Häuptlingswesens und dessen Finanzierung für Korruption und Vetternwirtschaft. Gleichzeitig ist das fidschianische Häuptlingswesen jedoch eine entscheidende Quelle ihrer kulturellen Identität. Jeglicher Eingriff, vor allem aber Schwächungen des Häuptlingswesens werden daher als potentielle Gefahr wahrgenommen, den gesellschaftlichen Zusammenhalt und die eigene Position in der Gesellschaft zu untergraben. Die Militärregierung trennt hier zwischen dem Häuptlingswesen auf lokaler Ebene, welche es als kulturelles Erbe und Wertebasis zu erhalten und teilweise zu stärken gilt, gegenüber einem destruktiven Elitismus auf nationaler Ebene; eine Unterscheidung, die in der öffentlichen Wahrnehmung nur begrenzt geteilt wird. Gerade der GCC galt als Garant für die Unantastbarkeit des kollektiven Landbesitzes, dessen Wegfall nun Ungewissheiten verursacht. Auch wenn die Militärregierung beständig versichert, die Landbesitzfrage nicht antasten zu wollen, ist die individuelle Wahrnehmung seit Jahrzehnten geschult, Veränderungen im Landrecht und im Häuptlingswesen (unabhängig räumlicher Bezugsebenen) als Angriffe auf das kulturelle Selbstverständnis zu werten. An derartigen Maßnahmen der Regierung scheiden sich die Meinungen und die autochthone Gesellschaft entzweit sich. 
Ähnlich dichotom reagiert die Bevölkerung auf die Haltung der Militärregierung zur Kirche. Bainimarama, selbst Methodist, jedoch katholisch erzogen und verheiratet mit einer Katholikin, versucht durch die Beschneidung der politischen Macht der obersten Häuptlinge, gleichzeitig die Macht der methodistischen Kirche zurückzudrängen (Weir 2015: 168f.). 2009 verbot er die methodistische Kirchenkonferenz - eine seit 160 Jahren bestehende Institution, die zu politischen Verlautbarungen genutzt wurde - und sagte auch den nationalen Chor-Wettbewerb $\mathrm{ab}$, eine der beliebtesten und größten religiös-kulturellen Veranstaltungen Fidschis. Rebellierende Geistliche wurden mit der Begründung inhaftiert, sie würden ihre persönlichen politischen Ambitionen über den Frieden und die Stabilität der Nation stellen (Trnka 2011: 77). Auch hierbei ist eine Bewertung schwierig, gerade aufgrund der Pressezensur, die keinen öffentlichen Vernunftgebrauch zulässt und somit freie Meinungsbildung verhindert. So gibt es zwar Befürworter, die einen säkularen Staat wünschen, in welchem sich die (methodistische) Kirche nicht in den politischen Alltag einmischt. Gegenteilige Extrempositionen hingegen sehen in dem Umsturz 2006 einen katholischen Putsch (Newland 2009: 196-201).

Am 17. September 2014 wurden Wahlen abgehalten. Die zuvor ausgearbeitete Verfassung beendete das Zensuswahlrecht nach Region und Ethnie; jeder Erwachsene Nationalstaatsangehörige hat nunmehr das gleiche Stimmrecht. Bei den Wahlen erhielt Bainimaramas Partei FijiFirst 60\% der Stimmen. Fünf der sechs weiteren zugelassenen Parteien bemängelten Irregularitäten bei der Stimmauszählung (Naleba 2014) und Wettbewerbsnachteile wurden angekreidet, internationale Wahlbeobachter sprechen jedoch von weitestgehend fairen Wahlen. Eine Einschätzung käme noch zu früh, ob Fidschis schwierigste politische Zeiten nun zurückliegen und die Bewohner selbstbestimmt ihre gewünschten Handlungspfade einschlagen können.

\subsection{Exposition gegenüber Klimawandel und Naturgefahren}

Klimatische Veränderungen gelten als ein möglicher Auslöser gesellschaftlicher und kultureller Veränderungen. Klimahistoriker vermuten, schnelle Klimaveränderungen könnten Gesellschaften zum Kollaps gebracht haben (Diamond 2005). Auslöser der Erstbesiedlung des Pazifiks könnte ein Meeresspiegelanstieg gewesen sein, der Küstengebiete Südostasiens unbewohnbar machte; Phasen des Absinkens wiederum erhöhten die Bewohnbarkeit vieler Inseln (Nunn 2007: 31f., 40-42, 53-58). Beim Blick auf gesellschaftlichen und kulturellem Wandel und diesbezüglichen Auswirkungen auf menschliche Sicherheit sollte daher eine Betrachtung des regionalen Klimas, klimabedingter Naturgefahren und ihrer Veränderungen einbezogen werden.

Die Fidschi Inseln liegen etwa von $177^{\circ}$ östlicher bis $175^{\circ}$ westlicher Länge, und von $15^{\circ}$ bis $22^{\circ}$ südlicher Breite. Die je nach Zählweise 300 bis 900 tropischen Inseln sind zumeist vulkanischen Ursprungs und stark reliefiert. Die Hauptinsel Viti Levu vereinnahmt 68\% der Landesfläche von insgesamt etwa 
$18.300 \mathrm{~km}^{2}$. Die Niederschlagsmengen schwanken aufgrund des vulkanischen Reliefs erheblich. So ist die Ostseite Viti Levus regenreich, die Westseite und die kleineren Inseln sind hingegen vergleichsweise regenarm, auch wenn aride Monate auch hier die Ausnahme bilden, da der Regen ganzjährig mit einem Peak im Sommer fällt (Terry 2007: 9f.). Tropischer Regenwald bildet die natürliche Vegetation, sie ist jedoch durch die Kultivierung stark zurückgedrängt (Faust 2006: 39f.). Die meisten Agrarprodukte lassen sich ganzjährig anbauen, Zuckerrohr als wichtigstes Agrarexportgut gedeiht am besten in den regenärmeren Gebieten, die kulturell bedeutsame Pfefferwurzel yaqona, gleichnamig mit dem aus ihr gewonnenen Getränk, wächst besonders gut an Hängen in der Lomaiviti Inselgruppe, zu der auch Gau und Koro Island gehören, sowie auf Kadavu und Taveuni. Die Sommermonate von November bis April sind mit Durchschnittstemperaturen von $26^{\circ} \mathrm{C}$ bis $27^{\circ} \mathrm{C}$ keine $3^{\circ} \mathrm{C}$ wärmer als die Wintermonate (Agrawala et al. 2003: 11). Dennoch macht dieser Gradient den Sommer zur Zyklon-Saison.

Naturgefahren lassen sich in zwei Gruppen unterteilen, tektonische und klimabedingte. Tektonisch ausgelöste Naturgefahren wie Erdbeben oder Tsunamis werden in dieser Arbeit nur nachrangig berücksichtigt, auch wenn die Region aufgrund der Grenzlage zwischen pazifischer und australischer Platte tektonisch aktiv ist. So hinterließ ein durch ein Seebeben vor der Küste Samoas ausgelöster Tsunami im Oktober 2009 auch auf den nordöstlichen Inseln Fidschis Spuren. In Gesprächen hatten jedoch lediglich die Ältesten Kindheitserinnerungen an einen Tsunami Anfang der 1950er, den sie als Flutwelle bezeichneten. Größere Erdbeben wurden dagegen keine erfahren. Daher konzentriert sich diese Arbeit auf klimabedingte Naturgefahren und hierbei insbesondere auf die vielfach erfahrenen Zyklone.

\subsubsection{Tropische Wirbelstürme}

Ein tropischer Zyklon kombiniert äolische und hydrologische Prozesse und schlägt sich in Form von Stürmen, Starkniederschlägen mit diesbezüglichen Landabrutschen und Sedimentation, Überschwemmungen, Sturmfluten und Küstenerosion nieder. Oftmals ist die Zeit nach dem Zyklon von Trockenheit gekennzeichnet. Andere Naturgefahren kulminieren daher in ihm, weshalb er als pars pro toto für klimatische Naturgefahren insgesamt betrachtet werden kann. Betroffen sind ländliche sowie maritime Ressourcen, die physische Infrastruktur sowie teilweise die Unversehrtheit der Menschen selbst. Mit 122 Zyklonen, denen die Region Fidschi-Samoa-Tonga in 26 Jahren ausgesetzt war (Chand \& Walsh 2009: 3878), stellt diese Naturgefahr eine stetige Bedrohung der Fidschianer dar.

Zur Entstehung eines tropischen Zyklons benötigt es mehrerer Vorbedingungen. Damit es zu Verwirbelungen kommen kann muss ein Abstand von mindestens $3^{\circ}$ Entfernung zum Äquator gegeben sein, da sonst die Coriolis-Kraft nicht ausreicht. Zusätzlich benötigt ein Zyklon eine geringe Windscherung zwischen den bodennahen Luftschichten. Weiterhin benötigt es großer Meeresoberflächen 
mit Wassertemperaturen von über $26,5^{\circ} \mathrm{C}$ in bis zu $50 \mathrm{~m}$ Tiefe als Vorraussetzung für ein Tiefdruckgebiet mit hoher Luftfeuchtigkeit als Quelle latenter Wärmeenergie bei geringem Reibungswiderstand (Chand \& Walsh 2009: 3878, Terry 2007: 15-18).

Die Erfassung eines Zyklons, insbesondere die Früherkennung und Kategorisierung fällt Meteorologen trotz enormer technischer Fortschritte schwer (Landsea et al. 2006: 453) - so war Zyklon Tomas am 14. März 2010 instrumentell gerade wieder als tropische Depression herabgestuft, als parallel erste Dörfer im Nordosten Vanua Levus von diesem Zyklon der Stufe 4 (zweithöchste Kategorie) verwüstet wurden (NEOC 2010a; NEOC 2010b; OCHA 2010; sowie eigene Verfolgung diverser Radio- und Fernsehberichte). Auch die Instandhaltung der Hochtechnologie scheint nicht immer gewährleistet, so war Anfang der 1990er das für 12 pazifische Inselstaaten ausgerichtete Radar in Nadi, Fidschi drei Jahre lang defekt, - so auch während Zyklon Kina im Januar 1993, mit 21 Toten und einer Schadenssumme von 165 Millionen FJ-\$ einer der verheerendsten Zyklone Fidschis, - ehe es ersetzt wurde (Jagmohan 1993; Tiffany 1993a, Tiffany 1993b). Insbesondere Vorhersagen über einen Zyklon-Pfad sind immer noch mit enormen Unsicherheiten versehen, sodass Frühwarnsysteme mitunter erst wenige Tage oder nur Stunden vorher die Gefährdeten alarmieren, auch weil zu häufiger Fehlalarm die Handlungsbereitschaft senkt - so teilte kaum jemand meine Besorgnis hinsichtlich einer Tsunamiwarnung am 06. Februar 2013, auch wenn die Frühwarnung per SMS, Radio und Fernsehen rechtzeitig griff, sodass viele informiert waren.

\subsubsection{Physische Auswirkungen des Klimawandels}

Erhöhte Meeresoberflächentemperaturen beeinflussen mit hoher Wahrscheinlichkeit tropische Zyklone. Die Meeresoberflächentemperaturen und Lufttemperaturen der Fidschi Inseln sind seit 1910 um 0,6 bis $1,0^{\circ} \mathrm{C}$ gestiegen. Zudem haben sich die Anzahl und das Ausmaß von Zyklonen der höchsten Kategorien (4-5) erhöht und schwere Niederschläge zugenommen. Klimaszenarien sagen eine Verschärfung dieser Trends voraus (PACC 2009: 3-9). Die Auswirkungen von Zyklonen auf die Menschen sind abhängig von ihrer Häufigkeit, Lebensdauer und Intensität. Die Intensität beschreibt die höchsten bodennahen Windgeschwindigkeiten im Auge des Zyklon, mit denen Reichweite, Wellenhöhen und Niederschlagsmengen korrelieren (Mataki et al. 2008: 264, 267). Aufgrund der Schwierigkeiten bei ihrer Erkennung existieren Unsicherheiten, in wie fern sich Häufigkeit und Lebensdauer erhöhen werden (Caron \& Jones 2007: 82f., 91-93), ihre Intensität und somit ihr Zerstörungspotential - nimmt mit steigenden Temperaturen jedoch voraussichtlich zu. Die „Assessments of Impacts and Adaptation to Climate Change" modellieren eine Steigerung der Intensität um 2,5\% bis 10\% pro Grad Celsius (AIACC 2007: 13). Ein anderes, viel diskutiertes (Anthes et al. 2006: 62426; Emanuel 2005b: E13; IPCC 2007a: 304f., Landsea 2005: E11f.; Pielke 2005: 
E11) Modell suggeriert 5\% pro Grad und erhöhte Lebensdauer (Emanuel 2005a: 687f.). Ob sich die Zyklon-Saison im Zuge der globalen Erwärmung ausdehnt, ist nicht beschrieben.

Neben Temperaturerhöhungen potenziert auch der Anstieg des Meeresspiegels im Zuge des Klimawandels die Zerstörungskraft von Zyklonen. Durch höheren Wellengang auf höherem Ausgangsniveau sowie stärkerer Windgeschwindigkeiten verschlechtert sich die Ausgangsbasis gegenüber Sturmfluten. Während man global betrachtet von einem Anstieg von 13 bis $30 \mathrm{~cm}$ im letzten Jahrhundert ausgeht (Bittermann et al. 2013: 4), konnte eine Messstation in Suva aufgrund regionaler tektonischer Senkungsprozesse in den letzten Jahren jedoch bereits einen Anstieg von 0,57cm pro Jahr feststellen (AIACC 2007: 9f.).

Temperaturerhöhungen und Meeresspiegelanstieg setzen zudem die Korallen unter Stress. Diese reagieren sensitiv auf Temperaturveränderungen. Ihr Toleranzbereich liegt zwischen $22^{\circ} \mathrm{C}$ und $28^{\circ} \mathrm{C}$, im Sommer $1997 / 98$ wurden daher 60\% der pazifischen Korallen von der Bleiche erfasst, im Zuge des El Nino 2002 starben nahezu alle Korallen aufgrund der Rekordtemperaturen von über $30^{\circ} \mathrm{C}$ (seit Beginn der Temperaturaufzeichnungen von 1870). Ab 2040 wird es vermutlich jährlich zur Korallenbleiche kommen (Reeh \& Kreisel 2002: 23-25). Dadurch gehen die Fischbestände zurück, denen Korallenriffs als Lebensraum dienen und Tourismus und Fischerei leiden, zwei wichtige Wirtschaftszweige Fidschis. Die im Zuge der Industrialisierung erhöhte Konzentration von $\mathrm{CO}^{2}$ in der Atmosphäre von derzeit ca. 380ppm sorgt für eine Versauerung der Ozeane, die den Lebensraum der Korallen für Algen attraktiver machen. Bei 500ppm, was zu einer Temperaturerhöhung von etwa $3^{\circ} \mathrm{C}$ führen wird, werden Algen Korallen als dominante Spezies ersetzen. Doch auch bei geringeren Temperaturerhöhungen kann das Korallenwachstum unter Stress nicht mit dem Meeresspiegelanstieg mithalten und es kommt zur Bleiche (Hoegh-Guldberg et al. 2007: 1740f.).

Auch Mangrovenwälder, die Fischen zum Laichen dienen und einen geschützten Lebensraum für Jungfische bieten, aber den Menschen auch zur Gewinnung von Feuerholz, Bauholz oder zu medizinischen Zwecken dienen, stehen bei ansteigendem Meeresspiegel unter Stress, können diesen aber durch Sedimentakkumulation und ggf. Inlandswanderung weitestgehend kompensieren (Alongi 2008: $2-4,9)$. Unabhängig vom Klimawandel beeinträchtigen die Menschen ihre Korallenküsten durch die Abholzung der Mangrovenwälder. Zudem entsteht ein erhöhter Sedimentationseintrag im Zuge der Landwirtschaft und Abfallwirtschaft, der neben den Korallen auch als Nahrungsmittel dienenden Seegräsern zusetzt (Lauer \& Aswani 2010: 988).

Korallenriffe fungieren als Wellenbrecher, Mangroven festigen die Küste und dämpfen die Energie von Wellen (Alongi 2008: 6f., Field 1995: 75). Beide sensiblen Ökosysteme dienen somit als natürlicher Küstenschutz. Ihre Schwächung hat eine erhöhte Erosionsanfälligkeit zur Folge, die ohnehin auch eine direkte Folge des Meeresspiegels ist. Zyklone richten mehr Schaden an, was in einer Negativspirale zu vermehrter Sedimentation führt. Es kommt häufiger zu Überschwemmun- 
gen und zur Bodenversalzung mit entsprechend negativen Auswirkungen auf die Landwirtschaft und die Mangrovenwälder selbst (Agrawala 2003: 32-36). Insbesondere an Flussmündungen hingegen kommt es parallel zu vermehrtem Süßwassereintrag, der Korallen und Seegräsern derart zusetzt, dass diese mehrere Monate zur Erholung benötigen (Fischer, 19, Nukui).

Werden keine Maßnahmen ergriffen, sorgt der Klimawandel für eine erhöhte Exposition gegenüber Naturgefahren. In einem gesellschaftspolitischen Klima der Unsicherheit, geprägt von einer Transformation der Gesellschaft und keiner stabilen politischen Lage sind langfristige Anpassungsstrategien an unvermeidbare Veränderungen schwierig, welche der Klimawandel zweifelsohne mit sich bringen wird. Das sozio-politische Umfeld bestimmt demnach die Anfälligkeit gegenüber Naturgefahren. Ob im Zuge von Anpassungs- und Bewältigungsstrategien eine Diskriminierung nach ethnischen oder anderen Kriterien stattfindet, ist mir nicht bekannt.

\subsection{Gesellschaftliche Trends und Dynamiken}

Die fidschianische Gesellschaft befindet sich in einem beschleunigten Prozess des Wandels. Die einst lokale dörfliche Wertegemeinschaft, verortet über ein indigenes Häuptlingssystem und traditionellen Konzeptionen von Land als vanua, ist heutzutage einem globalisierten Wettbewerb ausgesetzt. Alleine in den letzten 40 Jahren ist das Bruttonationaleinkommen preisbereinigt ${ }^{40}$ seit 1974 von ca. 2.381US-\$ auf 3.899US-\$ pro Kopf gestiegen (eigene Berechnungen nach FIBOS 2014a; UN Statistics Division 2015). Land-, Forst- und Fischereiwirtschaft trugen 2013 insgesamt nur noch 12,6\% dazu bei, der Bergbau war mit 0,4\% unbedeutend. Das verarbeitende Gewerbe machte $18,7 \%$ aus, sodass mehr als zwei Drittel des BNE auf den Dienstleistungs- und Informationssektor fielen (FIBOS 2014a). Die internationale Vernetzung zeigt sich an der Außenhandelsquote, die bei 120,6\% (FIBOS 2014b) liegt. ${ }^{41}$

Diese Wirtschaftszahlen suggerieren, Fidschi entwickle sich von einer Agrarzu einer Dienstleistungs- und Wissensgesellschaft. Doch nur 13,9\% der Gesamtbevölkerung bzw. 35,5\% der Erwerbsbevölkerung gehen überhaupt einer Lohnarbeit nach (FIBOS 2009: 4f., 10; FIBOS 2012: 105). Der überwiegende Teil der arbeitenden Bevölkerung ist in semi-subsistenter Landwirtschaft aktiv. Etwa 40.000 Fidschianer sind in der Zuckerrohrwirtschaft beschäftigt, jedoch größtenteils selbstständig. Es existieren knapp 20.000 unabhängige - bzw. zumeist in Korporativen vernetzte - Zuckerrohrfarmen von einer Durchschnittsgröße von 4,6ha, die vor allem für Indo-Fidschianer die Basis des Lebensunterhalts darstellen. Obwohl Zuckerrohr nur 6\% des BNE ausmacht, ist mehr als ein Viertel der

\footnotetext{
40 Preise bereinigt von Schwankungen des Verbraucherpreisindexes bzw. Inflation in Werten von 2005.

${ }^{41}$ Die deutsche Außenhandelsquote (Importe plus Exporte im Vergleich zum BNE) lag 2013 zum Vergleich bei 72,7\% (GTAI 2014: 3).
} 
Bevölkerung auf dieses Standbein angewiesen (Chand 2011: 349; Narayan \& Prasad 2003: 16f.).

Städtisches Leben und Arbeiten übt auf viele Fidschianer, insbesondere Frauen, einen hohen Reiz aus. Gerade im Tourismusbereich, mit etwa 25,1\% des BIP im Jahre 2013 Fidschis größter Wirtschaftszweig (eigene Berechnung nach FIBOS 2014a, FIBOS 2014c), sind überdurchschnittlich viele Frauen beschäftigt. Seit den 1960er Jahren, also mit der Aufhebung von Binnenwanderungsbeschränkungen, verzeichnen die ländlichen Regionen ein negatives Wanderungssaldo. Insbesondere in entlegenen Regionen wie der Lau-Gruppe ist die Abwanderung höher als das natürliche Bevölkerungswachstum, sodass die Bevölkerung der Provinz schrumpft (Bedford 1985: 333f., FIBOS 2008: 2f.) Mit Wachstumsraten städtischer Bevölkerung von 2,6\% bzw. 1,6\% pro Jahr während der letzten beiden Dekaden verzeichnet Fidschi eine weltweit hohe Verstädterungsrate und die Quote stieg von 40\% im Jahre 1986 auf 51\% im Jahre 2007 (Faust 1996: 32; FIBOS 2008: 3, 28). Die Binnenmigration schlägt sich in den Altersstrukturen der jeweiligen Provinzen nieder. Da vor allem junge Erwachsene - und dabei überproportional viele Frauen - migrieren, gibt es in ländlichen Räumen ein Bevölkerungsdefizit dieser Bevölkerungsgruppe bzw. einen Überschuss selbiger in städtischen Regionen (siehe Abb. 3).

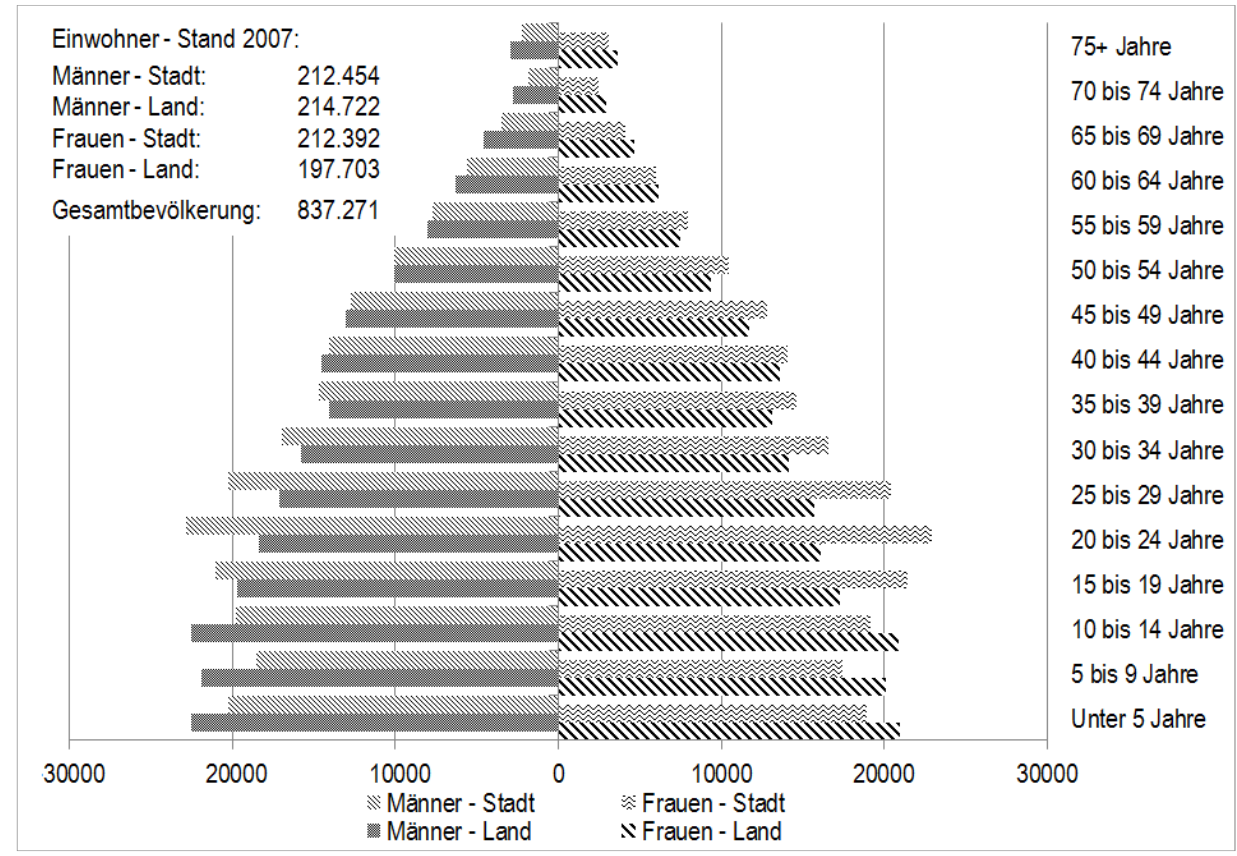

Abb. 3: Vergleich ruraler und urbaner Bevölkerungspyramiden (eigene Darstellung nach FIBOS 2008: 47) 
Nichtsdestotrotz erfüllt sich nicht für alle der Traum eines besseren Lebens in der Stadt. Insgesamt gelten 8,6\% bzw. nach ILO-Kriterien 11,9\% der Bevölkerung als arbeitslos. Die städtische Arbeitslosenquote liegt bei 10,5\% bzw. bei 13,8\%, bei städtischen Frauen gar bei 14,8\% bzw. 20,0\% (FIBOS 2009: 5). Vor 20 Jahren noch eher als Randphänomen wahrgenommen (Faust 1996: 25), prägen informelle Siedlungen zusehends das Stadtbild. Als negativ bewertete Begleiterscheinungen städtischer Lebensweisen wie Obdachlosigkeit, Verwahrlosung von Kindern und Senioren, Drogenmissbrauch oder organisierte Prostitution und Kriminalität sind weitestgehend neu und unbekannt für die zumeist dörflich geprägten Menschen, sodass der Gesellschaft der Umgang mit diesen jungen Phänomenen schwerfällt (Taylor 2005: 119f.). Die Urbanisierung führt in diesem Zuge zu einer Stärkung christlicher Institutionen, da religiöse Gemeinschaften in den Städten Halt bieten als Ersatz für den Wegfall dörflicher Vertrautheit (Newland 2009: 188f.).

Viele Fidschianer scheitern in der Stadt oder benötigen mehrere Anläufe, sodass viele immer wieder in ihre Heimatdörfer zurückziehen und die Migration als Zirkulationsbewegung (Zelinsky 1971: 225-226) verstanden werden muss. Auch mit dem Ausscheiden aus dem Erwerbsalter ziehen viele in ihr Heimatdorf zurück. Aufgrund des einfacheren Zugangs zu Land können sich insbesondere männliche iTaukei im Falle eines Scheiterns in der Stadt in die dörfliche Subsistenzwirtschaft zurückziehen. Die Dörfer dienen damit als Auffangbecken und stellen das zentrale soziale Sicherungsnetz dar. Bei Bedarf könnten die Subsistenzgemeinschaften autark das (Über-)leben sichern, sodass die Dörfer als Rückzugsräume abseits eines globalisierten Wettbewerbs als Alternative gegen Arbeitslosigkeit (Stiglitz 2001: xi) dienen. Auch wenn die Subsistenzwirtschaft nur mit 2,7\% im BNE veranschlagt ist (FIBOS 2014a), bildet sie mit 230.410 Beschäftigten (FIBOS 2011: 15) weiterhin das Rückgrat der Gesellschaft, insbesondere der iTaukei. Da gemäß globalisierter Wettbewerbslogik Arbeitslosigkeit außerhalb der Kontrolle und Einflussnahme des einzelnen Indiviuums einer städtischindustriellen Gesellschaft liegt, ist die Zirkulation in den Wanderungsbewegungen als Stärke zu betrachten. Die sozialen Netzwerke schaffen eine Diversität an Handlungsmöglichkeiten, sich sowohl in globalisierten markwirtschaftlichen Verhältnissen durchzusetzen versuchen zu können, als auch ins Heimatdorf zurückkehren zu können und daher stets nur weich Fallen zu müssen. Dies erklärt, warum zwar über 31\% der Gesamtbevölkerung bzw. 43\% der ländlichen Bevölkerung unter der Armutsgrenze lebt (FIBOS 2011: 87), Fidschi jedoch frei von Hunger und sonstigen Kennzeichen extremer Verwundbarkeit ist.

Trotz der Attraktivität einer Urbanität wird daher gerade seitens der iTaukei das Dörfliche nicht nur als Ideal hochgehalten, sondern seitens Auswärtiger bewusst gestärkt. Der Kontakt zur Heimat wird aufrechterhalten und die Beziehungen beständig über Gaben bzw. Rücküberweisungen reproduziert. Gerade auch Fidschianer in Übersee halten die Bande aufrecht. Leistungen und Gaben fließen dabei reziprok. So werden auch im Dorf hergestellte Gaben, die oftmals einen hohen symbolischen Wert besitzen (beispielsweise handgeflochtene Matten oder 
yaqona), an die außerhalb lebenden verschickt. ${ }^{42}$ Viele Städter lassen - auch abgesehen von möglichen ökonomischen Zwängen - ihre Kinder im Dorf aufwachsen, damit diese in den Genuss des „wahren“ Fidschis kommen und sich ihrer Identität bewusst werden, was sich ebenfalls in den Alterskohorten ländlicher und städtischer Bevölkerung (siehe Abb. 3) ausdrückt.

Der Abhängigkeitsindex gibt das Verhältnis nicht-arbeitsfähiger zu arbeitsfähiger Bevölkerung an. Da das Renteneinstiegsalter bei 55 Jahren liegt, gelten einer vereinfachten Rechnung zufolge über 55jährige und unter 15jährige als abhängig. Während in den Städten jeder Arbeitsfähige 0,60 Menschen mitversorgt, müssen auf dem Lande 0,76 Menschen pro Arbeitsfähigem versorgt werden (eigene Berechnung nach FIBOS 2008: 47). Da solche Abhängigkeitswerte als Indiz für Verwundbarkeit gelten (Adger et al. 2004: 69; Sofer 2009: 354), sehen ländliche Regionen innerhalb Fidschis besonders verwundbar aus. Dies wäre jedoch eine Fehlinterpretation. Stattdessen zeugen die Zahlen aufgrund der starken StadtLand-Interaktionen wie Zirkulationsbewegungen und Rücküberweisungen für Handlungsstrategien zur Gewinnung menschlicher Sicherheit sowohl der Dorfgemeinschaften als auch der städtischen Bevölkerung.

In diesem Übersichtskapitel wurden in einer plakativen Zusammenstellung Konstanten und Veränderungen der Gesellschaft Fidschis als kulturell bedingt einzigartige Mischung dargestellt. Die Tiefe und Komplexität des Wandels wird dabei als Transformationsprozess verstanden. Im nachfolgenden empirischen Teil der Arbeit werden die Auswirkungen der Transformation auf die Handlungsmöglichkeiten der iTaukei zur Sicherung ihrer Lebensqualität lokal anhand Studien dreier Dörfer erörtert.

\footnotetext{
${ }^{42}$ Eine groß angelegte Haushaltsstudie über den Zeitraum 2008-2009 erhob die Höhe von ausländischen und inländischen Geldsendungen auf 3,8\% bzw. 1,1\% und materielle Gaben (nicht nach Inund Ausland differenziert) auf 3,5\% des durchschnittlichen Haushaltseinkommens. Weitere Berechnungen legen jedoch nahe, dass ihr Gesamtwert insgesamt nicht bei etwa 8,4\% des Einkommens liege, sondern um bis zu 19 Prozentpunkte unterschätzt wird (FIBOS 2011: 13-25).
} 



\section{Empirie - Darstellung und Analyse}

Dieser Abschnitt ist das empirische Herzstück der Dissertation. Er widmet sich der Vorstellung und Analyse der vorwiegend partizipativ erhobenen Befunde aus drei Untersuchungsdörfern. Über einen kritischen Abgleich mit relevanter Literatur werden die eigenen Interpretationen trianguliert und abgesichert.

Die beleuchteten Aspekte der Lebenswelt fidschianischer Küstendorfbewohner wurden so ausgewählt, dass zunächst ein Einblick in die örtliche Lebensrealität gewährt wird. Aus dieser werden dann Ideale herausgezogen, die die kulturelle Gemeinschaft über ihre Alltagspraxis verwirklichen will. Darauf aufbauend können Bedrohungen des Sicherheitsgefühls veranschaulicht werden. Jedes Kapitel zeigt dabei Handlungsmuster auf, wie die lokale Bevölkerung ihre Lebensqualität $\mathrm{zu}$ sichern und $\mathrm{zu}$ steigern versucht. 



\section{Einführung in die untersuchte Lebenswelt - kulturelle Identität}

Die Empirie startet mit einer Vorstellung des politisch-administrativen Ordnungssystems Fidschis. Dies kennzeichnet eine konfliktbehaftete Parallelstruktur von Häuptlings- und Regierungswesen. Anschließend werden die drei Hauptuntersuchungsdörfer strukturiert nach ihrer soziokulturellen, politisch-administrativen und marktwirtschaftlichen Einbettung vorgestellt. Dabei wird untersucht, ob und wie sich ein gesellschaftlicher Wandel gestaltet und dieser sich auf die kulturelle Identität als Quelle von Lebensqualität auswirkt. Drei Exkurse zu Weihnachtsfesten in den jeweiligen Dörfern sowie ein Exkurs zu Familienstrukturen runden das Bild ab.

\subsection{Politisch-administrative Ordnungseinheiten und soziale Identitäten}

Fidschi ist von einem institutionellen Pluralismus geprägt. Das Häuptlingswesen und die staatliche Regierung gliedern Fidschi in politisch-administrativer Hinsicht. Beide Systeme existieren weitestgehend losgelöst von einander. Paradoxerweise führt das Nebeneinander jedoch nicht zu einer friedlichen Koexistenz. Auf unterschiedlichsten Ebenen überschneiden sich stattdessen Zuständigkeiten, sodass sich die beiden Systeme dialektisch gegenüber stehen.

\subsubsection{Häuptlings- und Staatswesen}

Der Nationalstaat mit zentraler Regierung unterteilt sich in vier Divisionen, welche sich in 14 Provinzen untergliedern. Jede Provinz wiederum besteht aus zahlreichen Distrikten. Eine im Regelfall einstellige Zahl an Dörfern bildet den Distrikt. Der gewählte Dorfvorsteher, turaga ni koro genannt, kommuniziert im Namen des Dorfes mit den staatlichen Institutionen wie die Distriktverwaltung und regionalen Vertretern der Ministerien.

Die obersten Häuptlinge einer jeden Provinz bilden den Großen Häuptlingsrat, eine Kammer der Nationalregierung, welche jedoch 2009 seitens der Übergangsregierung aufgelöst wurde. Seither ist das Häuptlingswesen auf staatlicher Ebene nicht mehr repräsentiert. Die staatliche territoriale Gliederung ist nicht konform mit den historischen Bündnissen und Beziehungen, welche das Häuptlingswesen abbildet. Hiernach bilden drei Stammeskonföderationen die oberste Gliederungseinheit, deren Machtbasen im Osten des Landes sitzen. Die westlichen Inselgruppen sowie die Westseite Viti Levus fühlen sich in diesem Dreiklang unterrepräsentiert und es gibt Bestrebungen, eine vierte Konföderation zu gründen. Keine aktuelle oder angedachte zukünftige Grenzziehung spiegelt jene der staatlich-administrativen Divisionen wider (siehe Karte 1). Mit dem Wegfall des großen Häuptlingsrats haben die Provinzen keine formale Rolle mehr im Aufbau des Häuptlingssystems; unterhalb der Konföderationen stehen über 200 vanua, Stammeszusammenschlüsse. Ihre Grenzen stimmen selten mit denen der Distrikte 
überein. Die yavusa, Stämme, welche sich zu vanua zusammenschließen, sind in der Regel Dorfgemeinschaften. Manche Dörfer können jedoch aus mehreren yavusa bestehen. Die yavusa untergliedern sich in mehrere mataqali, Klans, welche wiederum aus einem oder mehreren tokatoka, Großfamilien, bestehen. Dieses formale Gliederungssystem ist aufgrund der kolonialstaatlichen Festschreibung von Landbesitz auf mataqali-Ebene (siehe Kap. 5.1.3) äußerst starr. Über die Generationen kommt es de facto zu Wanderungen, Spaltungen, Auflösungen und Neubildungen, die kaum in das staatliche System integriert werden können.

Jedem mataqali, bzw. je nach Region jedem tokatoka, wird eine bestimmte Funktion in der Gemeinschaft zugewiesen. Die gängigen Funktionen sind hierarchisch geordnet: turaga, Häuptling, sauturaga, Häuptlingsmacher, mata ni vanua, Sprecher des Häuptlings (wortwörtlich: Auge des Landes), bete, Priester bzw. Diener, bati, Krieger, matai, Spezialist (z.B. als mataisau, Zimmermann) und dau, Begabter (z.B. als gonedau, Fischer oder dau ni vucu, Poet). In manchen Regionen hat jeder mataqali ein Oberhaupt, welches einen Adelstitel trägt. In vielen Regionen können nur Männer Häuptling sein. Mit der jeweiligen Funktion gehen für die Gruppenmitglieder Rechte und Pflichten einher. Da Dorfgemeinschaften jedoch oftmals nur aus zwei bis sechs mataqali bzw. tokatoka bestehen und Funktionen doppelt besetzt sein können, werden bestimmte Aufgaben nicht Gruppen zugeordnet sondern von Individuen getragen. Sollte es im Rahmen des Häuptlingswesens erhöhten Diskussionsbedarf geben, ruft der mata ni vanua zum einem boca ni vanua, einer traditionellen Gemeindeversammlung. Ich selbst durfte mehrfach solchen Versammlungen beiwohnen.

\subsubsection{Die Großfamilie}

Tokatoka halten sehr eng zusammen. Man beschränkt sich im alltäglichen Zusammenleben bewusst nicht primär auf die Kernfamilie, sondern lebt explizit mit der Großfamilie zusammen. Abbildung 4 zeigt den Aufbau eines Haushalts, mit dem ich zusammenlebte. Ein Ehepaar bildet mit drei ihrer sieben Kinder und vier Enkeln einen Drei-Generationen-Haushalt, der auf zwei Häusern verteilt zusammen-

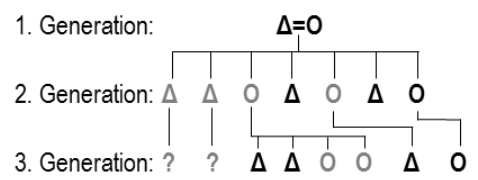

Erläuterung: $0=$ weiblich, $\Delta=$ männlich, grau $=$ außerhalb der Dorfgemeinschaft lebend Abb. 4: Zusammensetrung eines Drei-Generationen-Haushalts lebt. Die beiden ältesten Söhne sind verheiratet, die geschiedene älteste Tochter und die unverheiratete mittlere Tochter sind berufstätig und leben außerhalb der Gemeinde. Die jüngste, unverheiratete Tochter und die beiden unverheirateten, kinderlosen jüngsten Söhne hingegen leben aktuell im Haushalt. Die beiden jüngsten Enkel sind im Vorschulalter und werden von der jüngsten Tochter großgezogen, die sich zugleich um das ältere Ehepaar kümmert. 
Der älteste vor Ort lebende Enkel ist, wie die beiden vor Ort lebenden Söhne, Farmer und Fischer, während dessen Bruder noch zur Schule geht. Ihre beiden Schwestern leben bei der Mutter, die in einer Tourismusanlage arbeitet.

Aus dem Fallbeispiel wird deutlich, dass man nicht zwingend mit den leiblichen Eltern zusammenlebt und von diesen großgezogen wird, sondern die Aufgaben im Rahmen der Großfamilie aufgeteilt werden. Bezeichnenderweise wird alltagssprachlich nicht zwischen Vätern und Onkeln bzw. Müttern und Tanten unterschieden, sondern deren Alter entsprechend als ta levu (Großer Vater) bzw. ma levu (Große Mutter) bis ta lailai (Kleiner Vater) bzw. ma lailai (Kleine Mutter) angesprochen ( $\mathrm{ma}$ und ta sind Verniedlichungsformen, die auch mit Mama und Papa übersetzt werden könnten). Im Kleinkindsalter werden Kinder üblicherweise den leiblichen Müttern für mehrere Monate entzogen, um eindimensionale Mutter-Kind-Beziehung zu weiten. Dies dient dazu, Sicherheiten zu schaffen, dass Kinder sich auf viele Menschen im Rahmen des tokatoka verlassen. Risiken weniger, intensiver Abhängigkeiten wird so entgegengesteuert und Vertrauen in die Großfamilie geschaffen (Vielzahl an Gesprächen mit Müttern und Großmüttern in mehreren Dörfern).

Abbildung 5 bildet einen Versuch die Zusammensetzung eines gesamten tokatoka darzustellen. Dieser ist zugleich ein mataqali. Der tokatoka begründet sich auf die älteste, inzwischen verstorbene Tochter einer von auswärts stammenden Priesterfamilie. Diese heiratete jemanden aus der Dorfgemeinschaft und ihrer Verwandtschaft wurde daraufhin ein permanentes Bleiberecht geschenkt. Formale Landtitel wurden jedoch nicht umgeschrieben. Zur Ehrerbietung an die Begründerin des tokatoka und als Zeichen des Zugehörigkeitsgefühls zur Dorfgemeinschaft wurden viele Nachfahren nach der Priestertochter benannt. Gemeinhin wird deren drittältester Sohn (welcher für das Diagramm der Hauptinformant war) als aktuelles Familienoberhaupt gesehen. Dieser selbst möchte jedoch nicht mit Titel angesprochen werden - aus Respekt vor seinem im Dorf lebenden ta lailai (kleiner Vater bzw. Onkel), welcher mangels örtlichem Landtitel kein formales Amt innerhalb der Dorfgemeinschaft führen darf, sowie vor seinem in Suva lebendem ältesten Bruder.

Ein weiterer Beleg für die Bedeutung des tokatoka auf das Leben ist, dass man als tokatoka ein gemeinsames Konto führt. Wie der Beschuldigung eines Priesters an die Oberhäupter der tokatoka seines Dorfes zu entnehmen, „They all have a second bank account!" (Priester, 38), ist das versteckte Führen eines individuellen Kontos gesellschaftlich verpönt. Über mein enges Zusammenleben in mehreren Familien wurde deutlich, dass viele schon allein aus praktischen Gründen individuell oder als Kernfamilie ein weiteres Konto führen. Hier zeigt sich ein Widerspruch auf, wie man einerseits dem Ideal der Großfamilie entsprechen und andererseits eigenen Interessen nachkommen kann. Unklar ist, inwiefern dieser Wertekonflikt im Zuge des gesellschaftlichen Wandels erst entstand, oder aber sich nur anhand des Einzugs moderner Handlungsweisen wie Kontoführungen eindrucksvoll 




$\because$

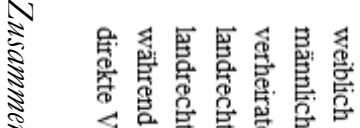

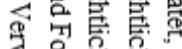

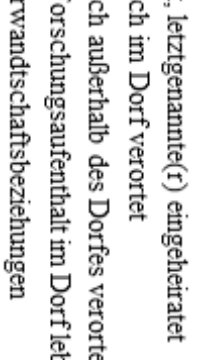

保

ฐ

$1 \triangleright 7$
$1>-$

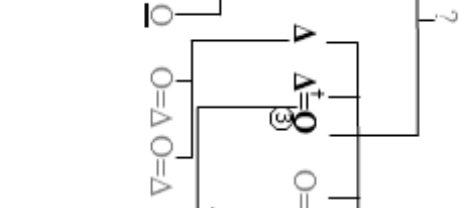

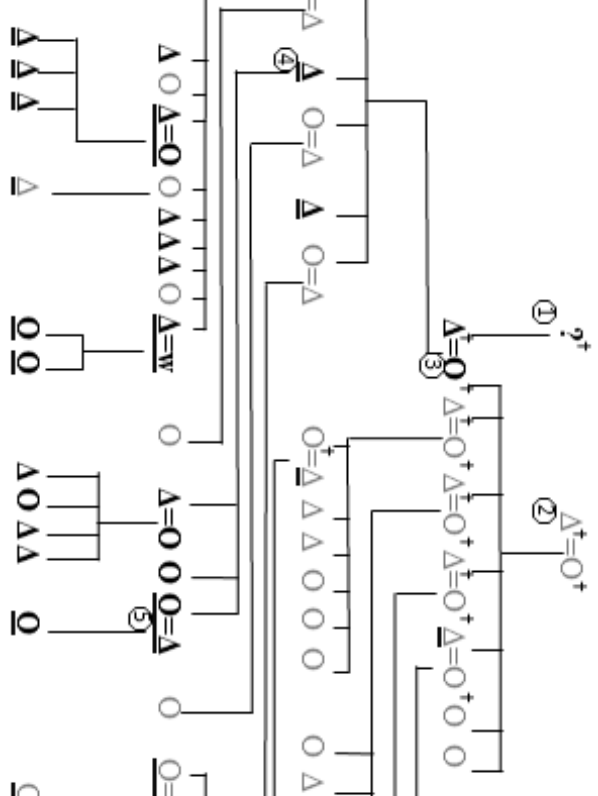

(요요

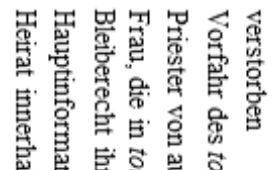



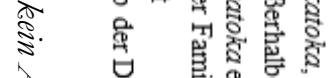

1

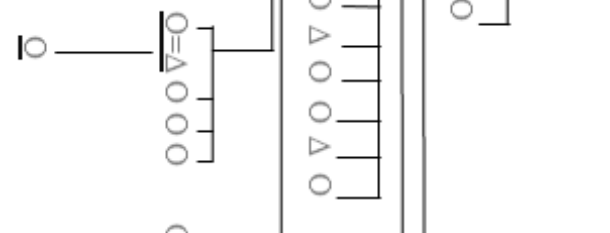

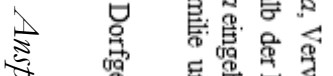



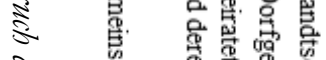

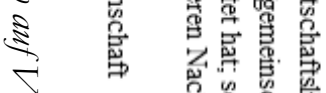



స:

$\frac{9}{20}$

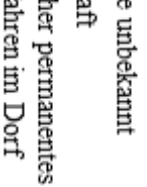

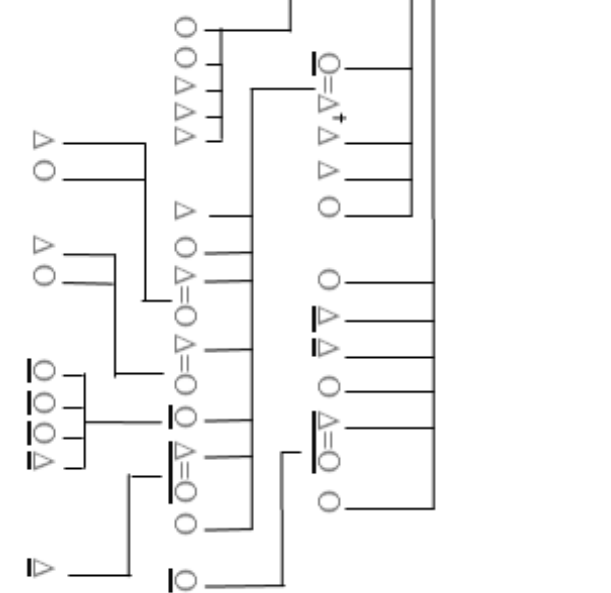


offenbart. Derartige Konflikte trüben jedoch nicht das grundsätzliche Vertrauen in die Familienangehörigen (siehe Tabelle 2). Neben dem Sparen als tokatoka führen die meisten Untersuchungsdörfer zusätzlich ein Gemeinschaftskonto auf yavusa-Ebene um sich vor Eventualitäten finanziell abzusichern.

Tabelle 2: Vertrauen zu bestimmten Personengruppen*

\begin{tabular}{|c|c|c|c|c|c|}
\hline $\begin{array}{l}\text { C1 To what extent do you trust the peo- } \\
\text { ple in the following categories? }\end{array}$ & 胥言 & 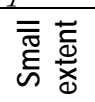 & 芯 䓂 & 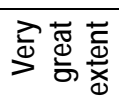 & \\
\hline Wertigkeit & 1 & 2 & 3 & 4 & $\emptyset$ \\
\hline family members & & 3 & 3 & 34 & 3,78 \\
\hline village community members (without family) & 4 & 25 & 7 & 3 & 2,23 \\
\hline village leader & 6 & 15 & 14 & 5 & 2,45 \\
\hline police & & 8 & 21 & 10 & 3,05 \\
\hline judges & 3 & 9 & 20 & 5 & 2,73 \\
\hline local government officials & & 5 & 18 & 16 & 3,28 \\
\hline national government officials & 2 & 4 & 23 & 10 & 3,05 \\
\hline members of non-governmental organisations & 6 & 11 & 16 & 6 & 2,56 \\
\hline strangers & 29 & 6 & 4 & 1 & 1,42 \\
\hline
\end{tabular}

\subsubsection{Dörfliches Zusammenleben}

Parallel zu den traditionellen Funktionen, die im Häuptlingswesen abgedeckt sind, gruppieren sich die Dorfbewohner zumeist unabhängig ihrer mataqaliZugehörigkeit in Komitees. Typische Gruppierungen sind Landwirtschafts-, Gesundheits- oder Schulkomitees, Sportklubs, Jugend- und Frauengruppen sowie kirchliche Vereine. In den 1970ern kam das Phänomen auf, sich als Dorfgemeinschaft in formale Gruppen und Komitees zu gliedern, gefördert seitens der Regierung und Nichtregierungsorganisationen (NGO). Viele Dorfbewohner sind in zwei bis drei Gruppen aktiv und verbringen mehr als den halben Tag im Rahmen ihrer Gruppen (Ergebnisse des Fragebogens, siehe Anhang). Die Gruppenzugehörigkeiten bilden für viele, jedoch nicht allen ihrer Mitglieder Quellen der sozialen Identität.

Frauen flechten als Gruppe Matten, welche für zeremonielle Zwecke benötigt werden. Frauen- und Jugendgruppen übernehmen in der Regel Reinigungsarbeiten und Landschaftspflege im Dorf, sofern vorhanden in Absprache mit dem Gesundheitskomitee. Die männliche Jugend bewirtschaftet zudem in organisierten Kleingruppen ihre Felder. Hier wird landwirtschaftliches Wissen weitergegeben, ausgetauscht und erarbeitet. Manche Farmer verlassen die Gruppe bereits in sehr jungen Jahren und bewirtschaften ihre Felder individuell, andere bleiben bis ins mittlere Alter in der Gruppe aktiv. Jugendgruppen unterliegen keinen strikten Altersbeschränkungen. Erreicht man ein Alter, in welchem man Enkel haben könnte, zieht man sich meist zurück. Die kirchlichen Gruppen können Chorgruppen sein, Bibelkreise, Messdiener, Gruppen zur Pflege und Instandhaltung der 
Gebäude, sich aber auch sozialen Belangen in der Kirchengemeinde widmen (Ergebnisse des Fragebogens, siehe Anhang).

Über den turaga ni koro kommunizieren die Gruppen mit regionalen Vertretern der Ministerien. Die Dorfbewohner erhalten so Beratungen und können Anträge zur materiellen und finanziellen Unterstützung dörflicher Entwicklungsprojekte stellen. Beispiele solcher Projekte wären der Ausbau oder die Renovierung der Gemeindehalle oder eines Schulgebäudes, Verbesserungen sanitärer Anlagen oder der Bau einer Küstenschutzmauer. Bei erfolgreichen Anträgen übernimmt die Regierung zumeist zwei Drittel der Materialkosten, die restliche Finanzierung sowie die Arbeitskraft stellt das Dorf. Über Fundraising-Partys wird das Gemeinschaftskonto entsprechend gefüllt. Die Koordination, welche Dorfgruppe welche Zuständigkeiten hat und im Falle eines Antrags das Gespür, welches Komitee bei welcher Ministeriumsvertretung die größte Erfolgsaussicht haben könnte, obliegt dem turaga ni koro. Er bildet damit eine Schlüsselfigur und das Amt genießt hohes Ansehen in der Gemeinde. Bei erfolgreichen, kofinanzierten Entwicklungsprojekten ist die Geberinstitution dabei für die Dorfbewohner zumeist von zweitrangiger Bedeutung und gerät in Vergessenheit (verschiedene offene Gespräche in allen drei Hauptuntersuchungsdörfern), während der zu jenem Zeitpunkt amtierende turaga ni koro hohe Anerkennung bekommt.

\subsubsection{Konfliktpotentiale aufgrund von institutionellem Pluralismus}

Die doppelte politisch-administrative Ordnung in ein Häuptlingswesen und staatlicher Institutionen verdeutlicht die Hybridität der Kultur. Die Zugehörigkeit zu einem mataqali und tokatoka bestimmt die Position in der Gemeinde und verweist auf die Aufgaben, Rechte und Pflichten einer Person. Über diese Klarheit ist man wesentlicher Bestandteil der Dorfgemeinschaft und die Zugehörigkeit ist elementar für die eigene Verankerung. Sie verleiht einem die kulturelle Identität und gibt Vertrauen und Sicherheit. Für das eigene Wohlbefinden ist das Häuptlingswesen daher von herausragender Bedeutung. Die für das gemeinschaftliche Zusammenleben zentralen formalen Gruppen tragen mitunter dazu bei, die sozio-kulturelle Identität der Dorfbewohner festigen.

Das staatliche Ordnungssystem hingegen wird als extern wahrgenommen, eine Identifikation findet kaum statt. So werden Rechtsstreitigkeiten versucht, „intern“ über das Häuptlingswesen zu lösen während man einer staatlichen Rechtsprechung mitunter kritisch gegenübersteht (siehe Tabelle 2 und Kap. 6.2.2). Die Verankerung des Landbesitzes auf mataqali-Ebene staatlicherseits wird einerseits als Sicherung und Schutz gewürdigt, andererseits verursacht das formal unflexible System mitunter Herausforderungen in der praktischen Handhabung.

Es existieren Unklarheiten über den Aufbau des staatlichen Systems und Zuständigkeiten innerhalb dieses Systems sind mitunter unbekannt. Unwissenheit 
über nationalstaatliche, wahldemokratische Abläufe ${ }^{43}$ zeugt von einem weitverbreiteten Desinteresse am Regierungsgeschehen. Dieses Unverständnis führt potenziell zu einer geringen Motivation an überlokal-politischer Teilhabe, sodass elementare Handlungsmöglichkeiten zur Verwirklichung eigener Ziele nach gutem Leben nicht ausgeschöpft werden. Ein diktatorisches Regime, in dem sich Gerüchte über staatliche Gewaltanwendung verbreiten, stößt auf Ablehnung und verschärft diese Gefahr der Abkehr von staatspolitischen Prozessen.

Theoretisch eröffnen sich durch das doppelte System politisch-administrativer Ordnung eine Vielzahl an Handlungsmöglichkeiten, am öffentlichen Leben teilzunehmen und sich selbst zu verwirklichen. Dieses Potential scheint jedoch kaum genutzt zu werden, stattdessen schränkt man sich in der Praxis nur auf das Häuptlingswesen ein. Diese Beschränkung gesellschaftspolitischen Handelns stellt im Sinne der konzeptionellen Rahmung eine Beschneidung von Verwirklichungschancen und damit eine Minderung der Lebensqualität dar. Im gesamten empirischen Abschnitt wird diesem Konfliktpotential zwischen den tendenziell widersprüchlichen Systemen eines traditionellen Häuptlingswesen und einer modernen Staatsführung Rechnung getragen. Zunächst wird die wirtschaftliche, sowie politisch administrative Einbettung mittels der Vorstellung der drei Hauptuntersuchungsdörfer veranschaulicht. Die abstrakten, formellen Systeme werden so über einen lokalen, konkreten Bezug mit Leben gefüllt, um deren Bedeutung im Alltag genauer aufzuzeigen.

\subsection{Malawai: Sozial-ökonomische Einblicke}

Das Dorf Malawai liegt auf Gau Island in der Lomaiviti-Inselgruppe. Der staatlichen, politisch-administrativen Ordnung zufolge gehört Malawai zur Provinz Lomaiviti in der Östlichen Division. Gau Island ist in drei Distrikten unterteilt. Malawai bildet mit Vione, Lekanai, Vanuaso, Nacavanadi und Lamiti den Distrikt Vanuaso. Gau Island liegt $90 \mathrm{~km}$ östlich der Hauptinsel Viti Levu und ist mit etwa $140 \mathrm{~km}^{2}$ bereits die fünftgrößte Insel. Auf dieser entlegenen Insel leben weniger als 3000 Menschen auf 16 Dörfern verteilt. Gau Island ist vulkanischen Ursprungs mit Höhen von über $600 \mathrm{~m}$ und einer heißen Quelle bei Nawaikama. Die Insel ist nahezu gänzlich von einem Korallenriff umschlossen. Im Landesinneren besteht ein weitestgehend intakter Regenwald (siehe Karte 2). Als Heimat des stark gefährdeten endemischen Sturmvogels Kacau ni Gau erhält die Insel vermehrt Aufmerksamkeit seitens internationaler ökologischer Organisationen.

\footnotetext{
${ }^{43}$ Eine gerne rezitierte Anekdote spielend Anfang der 1980er Jahre verdeutlicht das Unverständnis: Ratu Mara ist erster Premierminister des unabhängigen Fidschis und höchster Häuptling der TovataStammeskonföderation sowie sau ni vanua der Provinz Lau (Titel kann mit „Segen des Landes“ übersetzt werden). Bei einem Treffen auf Provinzebene in der Laugruppe wurde er gefragt, ob er verfügen würde, dass dessen Sohn Ratu Finau sein Nachfolger als Premierminister und sau ni vanua von Lau werde. Ratu Mara antwortete, er könne verfügen, dass sein Sohn sau ni vanua von Lau werde, jedoch müssten die Fidschianer über Wahlen selbst entscheiden, wer der nächste Premier wird und nicht er.
} 




Karte 2: Gau Island

Die Bewohner Malawais gehören drei verschiedenen christlichen Glaubensgemeinschaften an. Die Methodisten bilden die größte Gruppe und ihre Kirche steht im Dorf. Ein vakataua, ausgebildeter Laiepriester, leitet in der Regel gemeinsam mit wechselnden Dorfbewohnern die Messen. Ein Pfarrer, talatala, steht dem ge- 
samten Distrikt Vanuaso vor und initiiert Austäusche der sechs methodistischen Dorfgemeinschaften. Die All Nations als weitere Glaubensfraktion Malawais haben eine Kapelle 200m abseits des Dorfes und die zwei Mitglieder der Seventh Day Adventists gehören der Kirchengemeinde Vanuasos an. Zudem organisieren sich die Dorfbewohner in einer Frauengruppe, einer Jugendgruppe und Komitees für Elektrizität, Gesundheit und einen Shop. Weiterhin werden die Schule und eine maritime Schutzzone gemeinsam mit der benachbarten Dorfgemeinschaft Lamiti betreut.

\section{Exkurs 2: Weibnacbten 2009 - Malawai, Gau Island}

Vorweihnachtszeit. Ganz Malawai, ein kleines Dorf an der Ostküste Gau Islands, ist seit vielen Tagen in Aufruhr ob des großen Festes. Das ganz große Highlight der Weihnachtstage ist ein yavirau (traditionelle Fischtreibjagd) an Heilig Abend (Fink 2012, siehe Kap. 7.2.7). Hierfür sind viele auswärts lebende Verwandte extra angereist. Da oftmals nur eine Rumpffamile im Heimatdorf verbleibt, während die anderen meist in der Hauptstadt Suva, anderen Städten oder in touristischen Orten einer bezahlten Arbeit nachgehen oder zwecks Ausbildung auswärts leben, sind diese Wiedersehen sehr herzlich. Solche Heimreisen werden auch deshalb so gewertschätzt, weil sie große logistische und finanzielle Herausforderungen darstellen. Denn Gau Island wird nur etwa einmal monatlich per Fähre angesteuert. Ein zwangsweise längerer Heimaturlaub ist nur schwer mit dem Arbeitgeber zu arrangieren. Weiterhin können sich Fährfahrten witterungsbedingt verschieben oder gänzlich ausfallen- insbesondere, da Weihnachten in die Zyklon-Saison von Oktober bis April fällt. Die Planung startet zumeist weit im Voraus, kommt jedoch oftmals - wenn überhaupt - nur sehr spontan zustande. Die Fähre hält im Hafendorf Nawaikama auf der Malawai gegenüber liegenden Westseite Gau Islands. Da man mit viel Gepäck und Geschenken anreist und die einzige Straße für Autos nicht bis Malawai befahrbar ist, braucht man für den inselinternen Transport einen Platz in einem Fiberglassboot. Andere kommen direkt von Viti Levu aus per Fiberglassboot rüber, was noch teurer ist. Zur Weihnachtszeit ist dies zudem aus Sicherheitsgründen gesetzlich verboten, da die kleinen Boote, mit denen man über den offenen Ozean steuert, gerne überladen werden. Ich selbst setze am 20. Dezember mit einem Forschungsboot der Universität des Südpazifiks (USP) direkt nach Malawai über, gemeinsam mit zwei weiteren Wissenschaftlern, vier Forschungsassistenten und mehreren Einwohnern Malawais sowie des Nachbardorfes Lamiti.

Der beim yavirau gefangene Fisch bildet die Grundlage der Festmahle der kommenden vier Weihnachtstage. Denn Heilig Abend ist ein Donnerstag. Und fallen in Fidschi Feiertage auf ein Wochenende, so weden sie Wochentags nachgeholt. In Malawai feierte man daher durchgehend bis Montag. Glücklicherweise ist auch mehr als genug Fisch für die Festtage gefangen worden. Und das, obwohl sich viele Frauen individuell entscheiden insbesondere die, welche nach Malawai hineingeheiratet haben - gewisse Anteile an Verwandte in benachbarten Dörfern zu verschenken. Der verbliebene Fisch wird gemeinschaftlich innerhalb der mataqali hergerichtet. Um die Großgruppen bei den gemeinsamen Mahlzeiten - Frühstück, Mittag- und Abendessen - zu beherbergen sind eigens Unterkünfte aus Bambusrohren und Wellblechdächern errichtet worden. Man lädt dabei über 
die Tage Freunde und Verwandte aus den mataqali des Dorfes und der Nachbardörfer zu den Mahlzeiten ein, wird eingeladen oder lädt sich selbst ein.

Am späten Abend werden noch Weihnachtsmessen abgehalten. Die Methodisten, welche die größte Konfession bilden, gehen in ihre Kirche, die zwei Mitglieder der Seventh Day Adventists schließen sich ihnen an, da ihr Gotteshaus in Vanuaso für heute zu weit entfernt ist. Die Mitglieder der dritten Konfession des Dorfes, All Nations, feiern in ihrer Kapelle gut 200m außerhalb der Siedlung. Für die Methodisten stehen sonntags, sowie an beiden Weihnachtstagen jeweils drei Messen täglich an. Aufgrund der Essensvorbereitungen sowie sonstiger individueller Vorlieben nimmt jedoch kaum jemand an allen Messen teil.

\subsubsection{Marktwirtschaftliche Beriebungen}

Gau Islands Anlegestelle bei Nawaikama wird nur unregelmäßig etwa einmal monatlich per Fähre von Suva aus angesteuert. Malawai liegt auf der gegenüberliegenden Seite der Insel, sodass zusätzlich vor Ort Schnellboote zu chartern sind. Die Fähre ins Hafendorf Nawaikama kostet 70FJ \$ pro Person, das Chartern eines Fiberglassbootes von Nawaikama nach Malawai weitere 200 FJ\$. Innerhalb Gau Islands existiert kein bedeutender Handel und Grundnahrungsmittel lassen sich unter diesen Transportbedingungen nicht marktwirtschaftlich in Wert setzen. Gau Island ist innerhalb Fidschis Randgebiet und Malawai zählt damit zur Peripherie in der Peripherie, ist ein Paradebeispiel struktureller Ungunst.

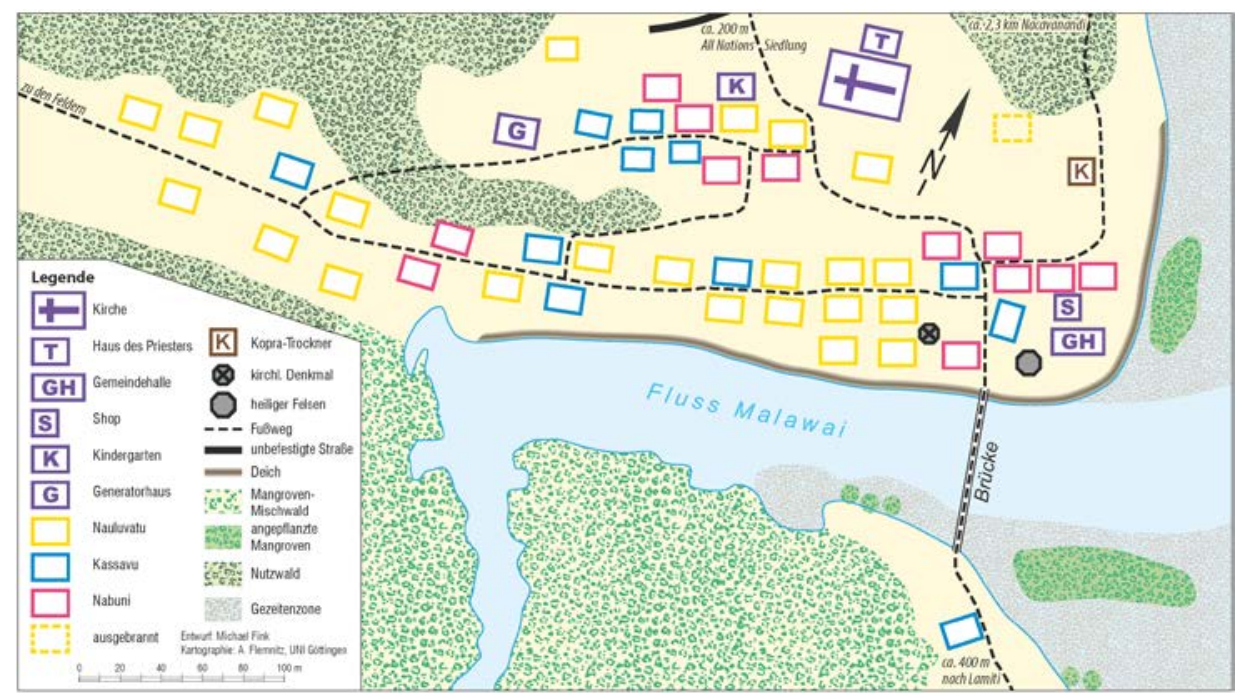

Karte 3: Malawai

Gemeinsam mit Taveuni und Kadavu gilt die Lomaiviti-Inselgruppe als bestes Anbaugebiet für yaqona. Die Wurzeln, welche man mindestens drei, besser jedoch sieben Jahre bis zur Ernte wachsen lässt, kosten für die Endverbraucher bis zu 
40FJ \$ pro kg. Selbst von der entlegenen Insel Gau aus lohnt sich der Transport zum größten Absatzmarkt nach Suva. Die von den Frauengruppen geflochtenen Matten stellen ein zweites Einkommensstandbein dar, mit sonstigen Agrarprodukten erzielt man allenfalls marginale Gewinne, sodass viele Farmer vom kommerziellen Anbau absehen. Fisch wird aufgrund seiner Verderblichkeit und der unzuverlässigen Transportbedingungen derzeit ebenfalls nicht ökonomisch genutzt. Der Preis von Kopra unterliegt starken Schwankungen. Phasenweise werden Kokosnüsse in geringem Umfang getrocknet und verkauft. Selbst die Jugend, welche marktwirtschaftlich noch kaum in Erscheinung tritt, sieht den schlechten Marktzugang als ein großes Problem an (siehe Abb. 6).
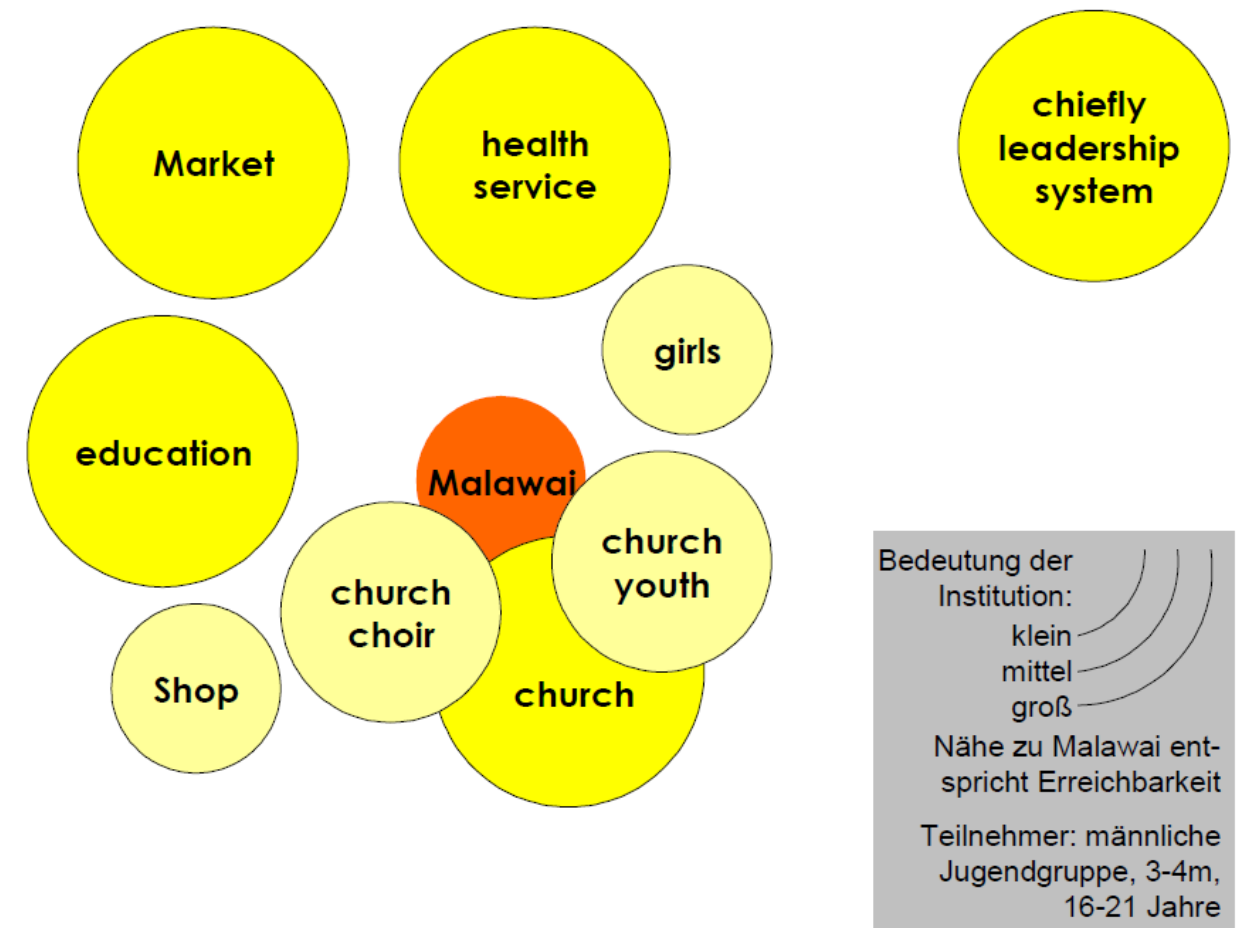

Abb. 6: Venn-Diagramm sozialer Institutionen der männlichen Jugend Malawais

Die Bewohner der Dorfgemeinschaften Gau Islands sind untereinander sehr vernetzt. Fast alle haben nahe Verwandte in den umliegenden Dorfgemeinden, die sie regelmäßig besuchen. Bei Besuchen werden stets Gastgeschenke überreicht. Dieser Brauch kann als reziproker Gabentausch fernab marktwirtschaftlicher Beziehungen verstanden werden und festigt den Zusammenhalt der Inselbewohner.

Die im Dorf lebenden tokatoka stellen nur Rumpffamilien dar. Der Großteil der Familienangehörigen lebt in Suva, manche leben und arbeiten in anderen Orten Fidschis, einige auch im Ausland, insbesondere Männer über das Militär. De- 
ren monetäre wie materielle Rückflüsse in die Dorfgemeinschaft stellen relativ zum Handel mit Agrarprodukten ein ähnlich großes wirtschaftliches Standbein dar. Auch hier ist man auf Reziprozität bedacht, wobei die verschickten Heimatprodukte einen eher ideellen denn finanziellen Wert haben, zumal der Transport die Produkte mitunter teurer macht, als ein Kauf vor Ort wäre.

\subsubsection{Häuptlingswabl und Hexerei}

Malawai, Lamiti, Nukuloa und Levuka gelten als älteste Gemeinschaften Gau Islands. Das Verhältnis der vier Dörfer zueinander lässt sich mittels Verwandtschaftsbeziehungen charakterisieren. Levuka nimmt demnach von Malawai aus gesehen die Stellung des großen Vaters ein, Nukuloa ist der kleine Vater und Lamiti ist der kleine Bruder. Diese Beziehungen spiegeln sich in Häuptlingshierarchien wider. Jedes Dorf hat einen eigenen Häuptling, der Tui Malawai ist Paramounthäuptling von Malawai und Lamiti, den Häuptlingen Levukas und Nukuloas jedoch formal untergeordnet. Über den Häuptlingen der vier Dörfer steht jener von Sawaieke als Häuptling von ganz Gau Island. Gau Island gehört zur Kubuna Konföderation deren Oberhaupt auf Bau Island sitzt.

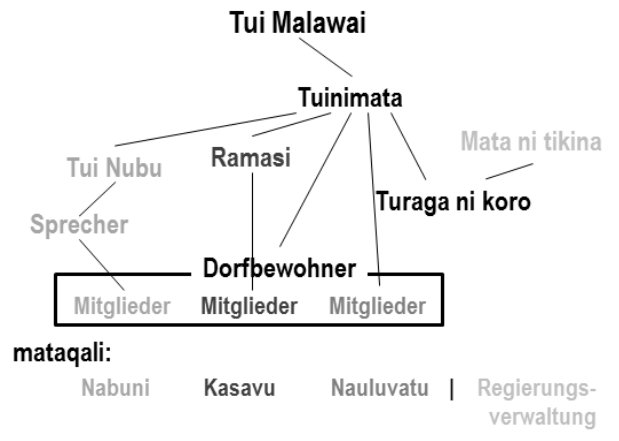

Abb. 7: Formaler Aufbau und Kommunikationsstruktur in Malawai

Malawai besteht aus sechs Großfamilien, tokatoka, die sich auf drei mataqali aufteilen. Zwei Klans, Kasavu und Nabuni sind bati, Krieger. Das Oberhaupt des mataqali Kasavu trägt den Titel Ramasi. Der mataqali Nabuni besteht aus zwei tokatoka. Der eine stellt das Klanoberhaupt, Tui Nubu, der andere seinen Sprecher. Der dritte Klan names Nauluvatu ist turaga, Häuptlingsklan. Dieser besteht aus drei tokatoka. Der erste stellt den Häuptling, Tui Malawai, der zweite dessen Sprecher, den mata ni vanua hier als Tuinimata betitelt - während der dritte keine formale Funktion hat (siehe Abb. 7). Die gängige Erzählung der anderen ist, dass dieser dritte tokatoka aus der Provinz Tailevu auswanderte und man ihn in ein bestehendes Gefüge aufgenommen hatte. Nach Ansicht des Familienältesten jenes tokatoka jedoch, sei er der wahre Paramounthäuptling von Malawai und Lamiti. Bei meiner Forschung in Dravuni, Tai Distrikt, Tailevu, erzählte man mir, man kenne die Person und hielt ihn ebenfalls für den Häuptling (siehe Kap. 6.4.1). Zu Zeiten Cakobaus agierte die Region Tai als rechte Hand Baus und stellte vor allem Krieger. Die Krieger aus Tai eroberten angrenzende Reiche für ihren König, so auch Gau Island. Viele Krieger ließen sich damals als Oberhäupter in den annektierten Dörfern nieder. 
Das Familienoberhaupt des umstrittenen tokatoka verfügt nach Selbstaussage als sau ni vanua (mögliche Übersetzung: „der Segen des Landes“) über Hexenkünste und kann daher Menschen durch Gedankenkraft töten. Der Fels vor seinem Haus zeuge davon, dass er die Wahrheit sage. „Wenn jemand gegen [seinen] Willen handelt, wird dieser auf dem Felsen liegen." Zum Beweis führt er den letzten offiziellen ${ }^{44}$ Tui Malawai an, der ohne seine Genehmigung ernannt wurde und 1979 verstarb, sowie die seither gescheiterte Nachfolge. Hexerei spielt im fidschianischen Dorfleben eine große Rolle. Yaqona wird eine spirituelle Kraft beigemessen (siehe Kap. 7) und wird nach fidschianischem Brauch nur in der Gemeinschaft getrunken. Jemandem zu unterstellen alleine zu trinken, ist gleichbedeutend, ihn der Hexerei zu bezichtigen. Da nur Menschen, die mit böser Magie im Bunde sind, alleine trinken (Toren 1999: 33; Turner 1986: 209), konsumiert der alte Mann yaqona bewusst alleine.

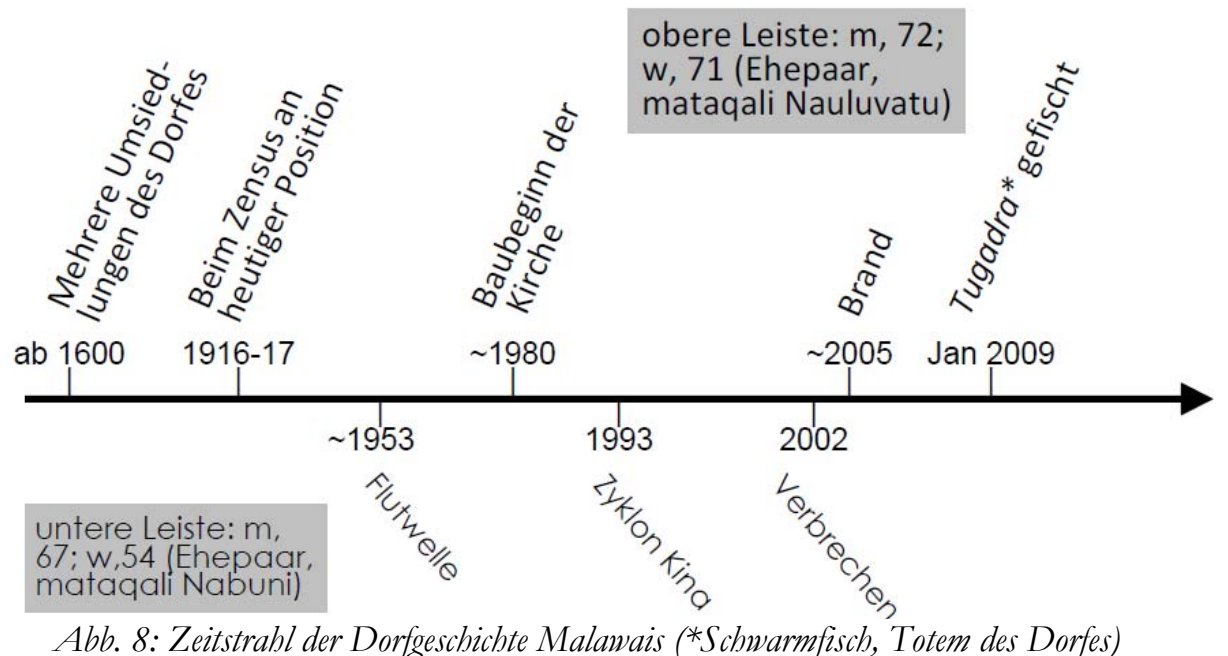

Als 2004 ein beliebter, angehender Häuptling verstarb und der sau ni vanua sich mit dessen Tod rühmte - oder aber ihm dieses zumindest nachgesagt wurde - eskalierte der Konflikt um das Häuptlingsamt. Mehrere Jugendliche unter Alkoholeinfluss versuchten, mit Macheten bewaffnet den Alten zu attakieren, sowie dessen Haus und das eines weiteren Familienmitglieds in Brand zu stecken. Da andere Dorfbewohner, insbesondere Familienmitglieder des Opfers, intervenierten, konnte der versuchte Totschlag verhindert werden, ein Haus brannte jedoch nieder. Der Hausbesitzer, welcher anschließend aus Angst nach Suva fortzog, war zudem talatala der Kirchengemeinde der Seventh-Day-Adventists. Religiös begründete Dispute mit der methodistischen Mehrheit im Vorfeld heizten die Situation zusätzlich ein. Der Fall wurde vor Gericht gebracht und die Täter zu bis zu

${ }^{44}$ Nach damaligem Stand des Interviews, Januar 2010. 
vier Jahren Haft verurteilt. ${ }^{45}$ Dieses schockierende Ereignis wurde bei zwei PRADiskussionen zur Dorfgeschichte als erstes erwähnt (mit abweichenden Jahreszahlen, siehe Abb. 8). Es war das bisher einzige Mal, dass Konflikte nicht innerhalb der Dorfgemeinschaft gelöst werden konnten und die Polizei eingeschaltet werden musste; ein Umstand, der als Schmach empfunden wird.

Dass der Konflikt um das Häuptlingsamt derart eskalieren konnte, unterstreicht die hohe Bedeutung des Häuptlingswesens auf die dörfliche Gemeinschaft. In sämtlichen drei Problem-Scoring-Gruppendiskussionen, ob nach Geschlecht, mataqali, religiöser Zugehörigkeit oder Jugend zusammengestellt, wurde das Machtvakuum des vakanten Häuptlingsamts als größtes Problem geschildert. Sämtliche weiteren Probleme sind sekundärer Natur; sobald ein Häuptling erfolgreich installiert ist, können sonstige Schwierigkeiten effizient angegangen werden. Zwei der drei Gruppen haben die Vakanz des Häuptlings lediglich als ursächliches Metaproblem geschildert, ohne es zu visualisieren (siehe Abb. 9). Weiterhin assoziierte eine weitere Jugendgruppe im Rahmen eines Venn-Diagramms den Umgang mit der ungelösten Situation als größte Herausforderung für die Gemeinschaft (siehe Abb. 6).

Kein Gesprächspartner strebt eine Abkehr vom Häuptlingswesen an. Die klar strukturierten Positionen innerhalb der Gemeinde und damit einhergehenden Rechte und Pflichten prägen die eigene Identität. Die Verankerung in der Gemeinde verleiht Status, Anerkennung und Respekt und gibt ein Gefühl der Sicherheit und Geborgenheit. Idealerweise weiß der Häuptling über die Ängste und Sorgen seiner Gemeindemitglieder und trifft weitsichtige Entscheidungen, die mögliche Konflikte im Vorfeld einschränken. Bei der Lösung eines Konfliktes ist er auf Konsens aus. Er vereint damit die Gemeinschaft und ist ihre moralische Instanz.

In der Alltagspraxis lassen sich sämtliche Meinungen und Wünsche aller Gemeindemitglieder nicht immer im Konsens vereinen. Manche Konfliktfelder werden daher aufgeschoben, um die Harmonie der Gemeinde nicht zu gefährden. Derartige Pattsituationen sind jedoch ebenfalls unbefriedigend. Können Probleme nicht ausgesessen werden, brodeln sie unter der Oberfläche, bis sie eskalieren, wie das Beispiel des Mordanschlags verdeutlicht. Daher wird der Häuptling legitimiert, harte Entscheidungen abseits des Konsens zu treffen, um die Gemeinschaft als solche voranzubringen. Ein Häuptling wird entsprechend respektiert und geschätzt, dass er Entscheidungen fällt, umgekehrt hat er die Konsequenzen (mit) zu verantworten. So bildet der Häuptling in jedem Dorf, das ich besuchte, eine Reizfigur. Alle sehen den Sinn des Häuptlings an und untermauern die Wichtigkeit seiner Funktion, Entscheidungen zu treffen, auf das die Gemeinschaft nicht stillsteht, sondern sich verbessert. Andererseits wird die jeweilige Person, welche das Häuptlingsamt bekleidet, von vielen als unglückliche Besetzung wahrgenommen.

\footnotetext{
${ }^{45}$ Da verschiedene Gerichtsdokumente über den Fall öffentlich zugänglich sind (Shameem 2005), erlaube ich mir, hierüber schreiben zu dürfen, ohne Persönlichkeitsrechte zu verletzen.
} 


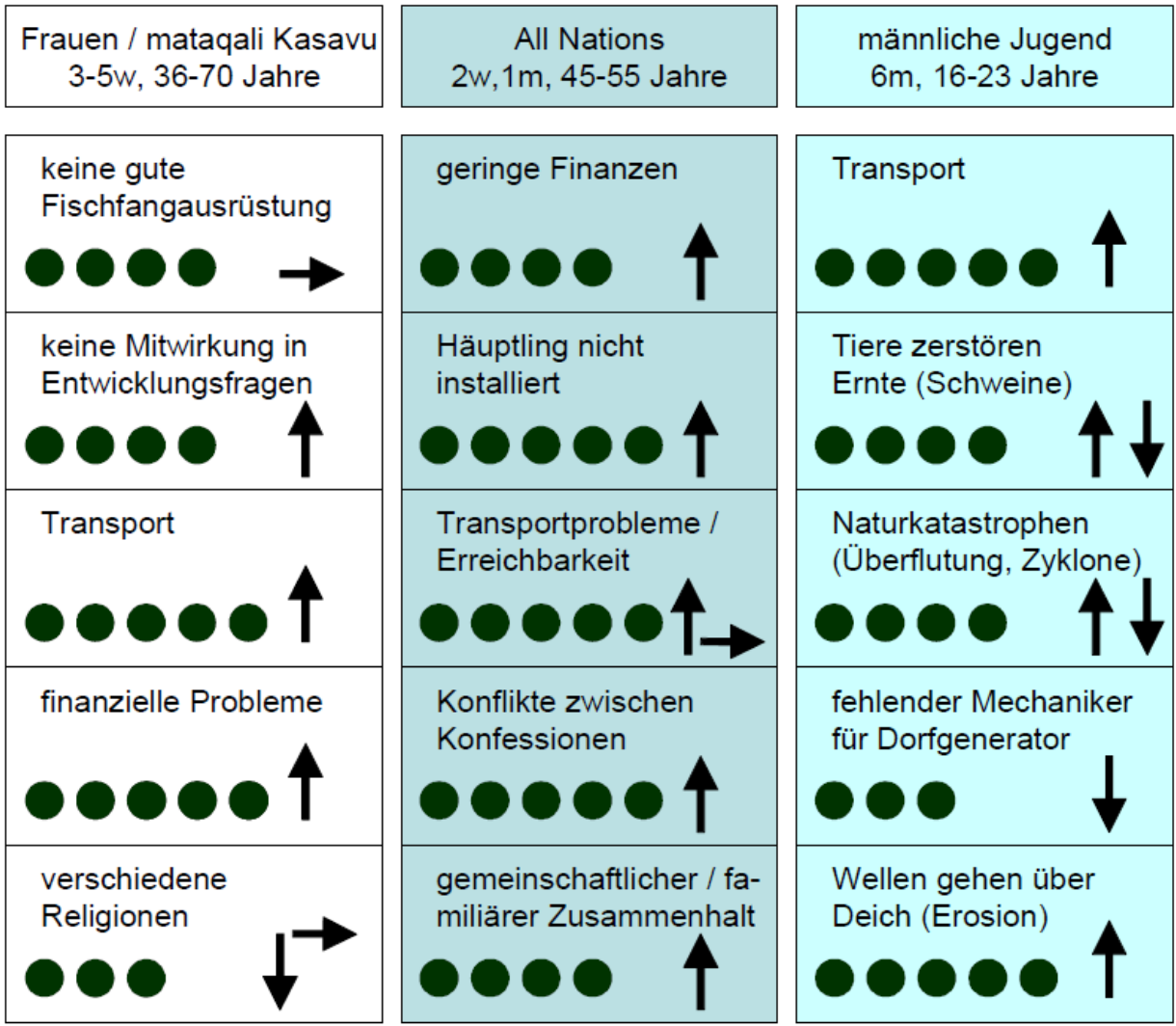

Abb. 9: Allgemeine Problem-Wertungen (Punkteanzabl zeigt Schwere an; Pfeile geben 10Jabres-Trend wieder, nach oben $=$ Verschärfung, zur Seite $=$ Stagnation, nach unten $=$ Verbesserung)

Bei der Wahl eines neuen Häuptlings wird eine der ältesten, männlichen Personen des Führungs-tokatoka auserkoren. Diese kann der älteste Bruder des vorherigen Oberhauptes sein, sein ältester Sohn, aber genauso gut ein Vetter, Neffe oder weiter entfernter Verwandter innerhalb des tokatoka. Der Tui Malawai wird vom mataqali Nabuni vorgeschlagen. Der mataqali Kasavu hat anschließend ein Vetorecht. Der mataqali Nauluvatu, aus dem der Häuptling kommt, hat keine formelle Mitwirkungsgewalt. Beratungen über die Wahl des Tui unterliegen hierbei einer Vielzahl weiterer Rituale, die den gemeinsamen Entscheidungsfindungsprozess regeln. Dieser Prozess dient dazu, dass jede Meinung berücksichtigt werden kann und zieht sich über Jahre. Ist einmal der Tui Malawai offiziell verkündet, behält er sein Amt bis zum Tode. Sollten geeignete Kandidaten außerhalb Malawais leben, oder aus sonstigen Gründen das Amt derzeit nicht antreten wollen, behilft man sich mit Interimslösungen. Seit 1979 der offizielle Tui Malawai verstarb, wurde das Dorf von verschiedenen Interims-Häuptlingen geführt. Diese genossen jedoch 
nicht den nötigen Respekt, sodass ihre Entscheidungen mitunter nicht anerkannt wurden. Dass die soziale Gemeinschaft erodiert, wenn den Weisungen des Häuptlings nicht gefolgt wird, sehen die Dorfbewohner als große Gefahr.

Es vergingen über 30 Jahre, ehe 2010 ein pensioniertes Mitglied der Häuptlingsfamilie nach jahrzehntelanger Arbeit auf internationalen Frachtschiffen zurück nach Fidschi kam und für das Häuptlingsamt in Malawai geworben werden konnte. Inzwischen wurde er im Rahmen vielfältiger Zeremonien zum Häuptling gekürt. Individuelle Gespräche während nachfolgender Aufenthalte im Januar 2012 und Januar 2013 in Malawai ergaben, dass die Dorfbewohner mit der Installation des neuen Häuptlings äußerst zufrieden sind. Während die Entscheidungen der Interims-Lösungen polarisierten, überwiegt nun die gemeinsame Freude über eine zentrale Leitfigur. Die Reproduktion der eigenen Traditionen stärkt die kulturelle Identität, welche eine Quelle für Vertrauen in die Gemeinschaft birgt und ihren Zusammenhalt stärkt. Der gesellschaftliche Wandel wird verantwortlich gemacht für die Entstehung des unbefriedigenden, jahrzehntelangen Machtvakuums. Durch das Hochhalten der eigenen Traditionen scheint es nun geschlossen.

\subsubsection{Migration als Chance und Risiko der Transformation}

Seitdem die staatlichen Möbilitätsbeschränkungen in den 1960er Jahren gelockert wurden, verlassen viele Bewohner entlegener Inseln ihre Dörfer in der Hoffnung auf Lohnarbeit auf Viti Levu oder in Übersee. Viele Ausgewanderte unterstützen die Dorfgemeinschaft mittels Rücküberweisungen (siehe Kap. 6.5, sowie Einzelgespräche und Auflistungen bedeutender Einzelpersonen ${ }^{40}$ ). Seither steht die Dorfgemeinschaft häufig vor der Abwägung, ob die Aussicht auf Bildungsmöglichkeiten und Lohnarbeit von Einzelpersonen den Verlust kompensiert, der mit dem jeweiligen Weggang einhergeht. Auswärtige können die soziale Nähe nicht aufrechterhalten und ihre traditionellen Funktionen in der Gemeinschaft nur unzureichend wahrnehmen. Entsprechend wird im engeren Familienkreis, sowie unter Berücksichtigung der Meinungen innerhalb der gesamten Dorfgemeinschaft entschieden, ob solche Jobmöglichkeiten im Einzelfall wahrgenommen werden. In den meisten Fällen werden individuelle Wünsche nach Migration jedoch nicht ausgeschlagen, sodass nur noch Rümpfe der Großfamilien dauerhaft in Malawai wohnen. So verlassen die meisten Jugendlichen im Anschluss an die achtjährige Grundschulzeit die Gemeinschaft zwecks Aus- und Weiterbildung. Viele lassen sich erst im Renteneintrittsalter wieder dauerhaft im Dorf nieder. Im erwerbsfähigen Alter zirkulieren Einzelpersonen oder Kernfamilien zwischen Arbeitsstandorten und - in Phasen der Erwerbslosigkeit - dem Heimatdorf. Während für die männliche Bevölkerung Farmarbeit im Dorf durchaus attraktiv ist, bleiben Frauen auch bei Arbeitslosigkeit gerne bei Verwandten in den Städten, sodass insbesondere Frauen zw. 15-45 Jahren unterrepräsentiert sind (siehe Abb. 3). Die unverheira-

46 Zur Wahrung von Persönlichkeitsrechten werden die entstandenen Listen nicht abgebildet. 
tete männliche Jugend übersteigt die Zahl lediger Frauen bei weitem, was als Problem wahrgenommen wird (siehe Abb. 6).

Die Herausforderungen bei der Häuptlingsnachfolge liegen in Malawai nun sowohl an einer unterschiedlichen geschichtlichen Auffassung, welche Großfamilie den Häuptling stellt, als auch angesichts der Abwesenheit geeigneter Kandidaten vor Ort. Darüber hinaus wird es für Dorfgemeinschaften wie Malawai schwieriger auszumachen, welche Qualitäten ein geeignetes Oberhaupt mitbringen sollte. So wird der Wunsch geäußert, dass er neben eigener Führungsstärke sich auch von nicht traditionell legitimierten Wissensinstanzen beraten lassen sollte. Denn mehrere Mitglieder der Dorfgemeinschaft haben eine universitäre Ausbildung genossen, sodass sich eine Bildungsschicht herauskristallisiert.

Gau Island gilt als globaler Hotspot der Biodiversität, insbesondere aufgrund eines intakten Regenwalds und als Heimat des endemischen Sturmvogels Kacau ni Gau. Der Vogel gilt als Totem einzelner Dörfer Gau Islands sowie der gesamten Insel. Aufgrund dieser Heiligkeit wird die Tierart gerne seitens der Inselbewohner beschützt. Hierzu sind auch internationale NGOs vor Ort aktiv; verschiedene Tierschutzorganisationen kooperieren zur Erhaltung der hoch gefährdeten Art mit der Dorfbevölkerung. Sie tragen so externes Wissen in die Gemeinschaft.

Die identitätsstiftende, traditionelle, funktionale Gliederung der Dorfgemeinschaft nach mataqali und tokatoka möchte man einerseits nicht aufgeben. Andererseits möchte man sich der Möglichkeiten nicht berauben, das Wissen moderner Institutionen gewinnbringend für sich zu nutzen. Wie Beratungsfunktionen in die traditionellen Kommunikationswege integriert werden können, bedarf einer hohen Sensibilität sämtlicher Akteure.

In der Zeit der Interimshäuptlinge waren manche als geeignet erscheinende Häuptlingskandidaten nicht vor Ort verfügbar, andere lehnten das Amt wiederum $\mathrm{ab}$, da die Bürde der Verantwortung in einem solch heterogenen, vielstimmigen Umfeld als zu schwer empfunden wurde. Das Beispiel der Häuptlingsfrage Malawais liefert Indizien, dass die gesellschaftliche Transformation als komplex, verwirrend und als Unsicherheitsfaktor wahrgenommen wird. Rückbesinnungen auf bzw. das Wiedererstarken von als ursprünglich wahrgenommener kultureller Handlungen - hier die Installation eines Häuptlings - stellen schwierig zu verwirklichende Wege dar, bergen aber ein Potential soziale Sicherheit zu erzeugen.

\subsection{Nabuna: Sozial-ökonomische Einblicke}

Nabuna liegt an der Nordküste Koro Islands. Auf Koro Island leben gut 4.000 Menschen in 14 Dörfern und mehreren weiteren kleinen Siedlungen. Genau wie Gau Island gehört Koro zur Provinz Lomaiviti. Die Insel ist ebenfalls vulkanischen Ursprungs, ist von einem Korallenriff umgeben und erreicht Höhen von über $500 \mathrm{~m}$. Die mit rund $105 \mathrm{~km}^{2}$ siebtgrößte Insel Fidschis liegt auf der Fährverbindung zwischen Suva - Savusavu, die die beiden größten Inseln miteinander verbindet (siehe Karte 4). 


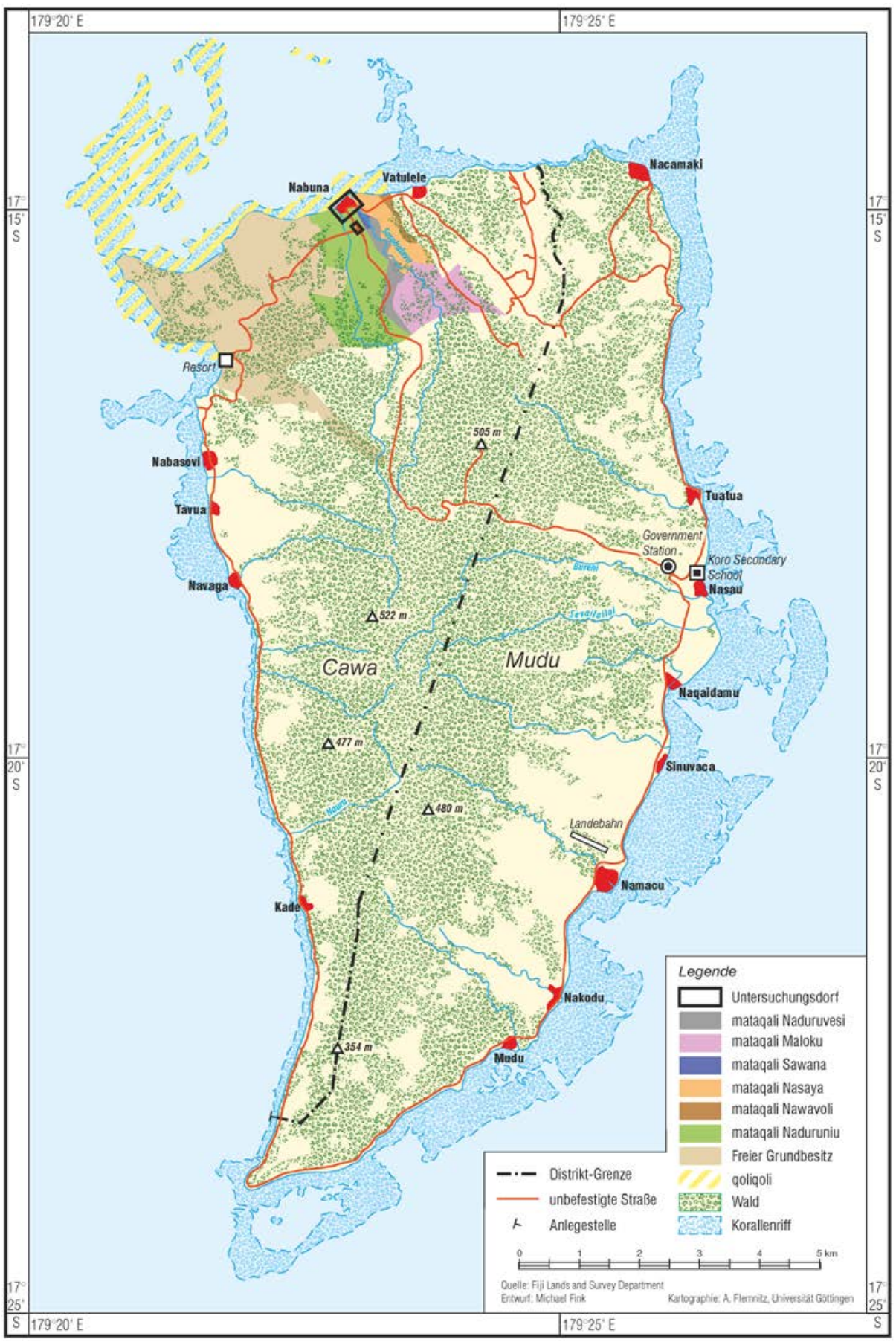

Karte 4: Koro Island 
Nabuna hat etwa 130 Einwohner. Zum Nachbardorf Vatulele bestehen besonders enge Beziehungen und der Tui Nabuna ist Paramounthäuptling beider Dörfer. Nacamaki und Nasau wurden von Nabuna aus gegründet. Alte Ortsnamen zeugen von der Beziehung, so hieß Nabuna früher „Matana Levu“ (großes Auge) und Nasau „Matana Lailai“ (kleines Auge). Im Zuge der Kolonialisierung und Christianisierung wurden die Orte umbenannt. Die Bewohner Nabunas halten den alten Namen und die angestammten Beziehungen bewusst aufrecht, beispielsweise trägt ein Schnellboot den alten Ortsnamen. Anders als Gau Island hat Koro Island keinen Häuptling, der für die gesamte Insel spricht. Die staatliche Verwaltung unterteilt Koro in zwei Distrikte und spaltet dabei die vier Dörfer auf.

Zwei christliche Glaubensgemeinschaften existieren in Nabuna. Die zahlenmäßig größere Gruppe bilden die Methodisten. Sie haben eine eigene Kirche im Dorf, einen talatala, der für Nabuna, dem Nachbardorf Vatulele und die benachbarte Siedlung Nagadro verantwortlich ist. Zudem ist ein vakataua ausschließlich für Nabuna aktiv. Als in den 1960er Jahren die Schotterstraße angelegt wurde, welche alle Dörfer Koros verbindet, kamen für ihren Bau viele Arbeiter von auBerhalb Koro Islands auf die Insel. Viele Fidschianer, insbesondere von der LauInselgruppe ließen sich anschließend dauerhaft in Siedlungen nieder und pachten Land von den Klans der Dorfgemeinschaften. Über Freundschaften und Eheschließungen, sowie kirchlich über gemeinsame Gottesdienste, Chorgruppen und Fundraising-Aktivitäten pflegen die Bewohner Nagadros vielfältige, intensive soziale Netzwerke mit den beiden Dorfgemeinschaften.

Weiterhin leben viele Katholiken in Nabuna und Vatulele und bilden eine Gemeinschaft. Diese spart auf ein eigenes Gotteshaus. Viele der Katholiken gehören dem mataqali Naduruniu an. Vor gut zehn Jahren haben mehrere Mitglieder eine eigene Siedlung namens Namilau rund 300m südwestlich des Hauptdorfes errichtet, sind jedoch weiterhin in der Dorfgemeinde verankert. Derzeit werden abwechselnd in Vatulele und Nabuna Privathäuser für die sonntäglichen Gottesdienste in Kapellen umfunktioniert. Ein Katechist leitet die Messen. Zu besonderen Anlässen fährt die Gemeinde nach Nacamaki, wo die einzige katholische Kirche Koro Islands steht und der einzige Priester der Insel wohnt.

Seit den 1970er Jahren gründeten die Dorfbewohner eine Frauengruppe, Jugendgruppen, einen Rugbyclub, Kommitees für Gesundheit und Landwirtschaft, sowie - zeitlich versetzt - für Elektrizität. Während die Mitgliedschaften normalerweise unabhängig von einer mataqali-Zugehörigkeit sind, existiert im mataqali Sawana eine auf Klanzugehörigkeit beschränkte Jugendgruppe. Während meiner Forschungszeit wurde ein Senior, der für seine Altersruhe in das Dorf zurückgekehrt ist, zum Ehrenmitglied ernannt. Ihr aktiver Kern sind jedoch Jugendliche und junge Erwachsene. Die Sawana-Jugendgruppe arbeitet sehr erfolgreich zusammen und kooperiert geschickt mit den lokalen Vertretern der Ministerien. Mittels staatlicher Unterstützung konnte so ein Billardtisch und ein Schnellboot angeschafft werden und Möglichkeiten der Schaffung eines Internetanschlusses werden derzeit diskutiert. Manche Jugendliche anderer mataqali würden der Grup- 
pe gerne formal beitreten, sind jedoch ausgeschlossen (Ergebnisse des Fragebogens, siehe Anhang). Im dörflichen Alltag wird äußerst viel Zeit im Rahmen der Gruppen verbracht. So trifft sich die Frauengruppe an bis zu drei Nachmittagen wöchentlich, was bis zu 60Std. monatlich bedeutet. Hauptsächlich werden in dieser Zeit gemeinsam Matten geflochten. Frauen, die schulpflichtige Kinder im Haushalt haben, sind zudem über das Schulkomitee in die Schulspeisung eingespannt. Ist man parallel noch in einer kirchlichen Gruppe aktiv, ist der Alltag stark von den Gruppen beherrscht.

Rugby ist der beliebteste Sport und Nabuna hat einen Rugby-Verein. Stehen Turniere an, trainieren die männlichen Spieler fast jeden Abend für ca. 2 Stunden. Tagsüber steht man teilweise als Gruppe auf den Feldern, um Gelder für Ausrüstung und Transport zu erwirtschaften. Koro Island ist fidschiweit bekannt für seine Ausnahmetalente. Ein Mitte 20jähriger Dorfbewohner durchlief die JugendNationalmannschaften und war für Turniere unter anderem in Frankreich und Japan. Er lebte und trainierte damals in Suva. Seinen Status als aufkommender Star konnte er jedoch schlecht verarbeiten. Als er vom Trainer des illegalen Drogenkonsums erwischt wurde, verbannte dieser ihn aus der Mannschaft. Er spielt weiterhin begeistert in der Dorfmannschaft, macht sich aber nur noch wenige Hoffnungen auf ein Comeback. Manche Familienmitglieder lasten ihm an, dass er seine Chance zu einem internationalen Rugby-Spieler vertan hat. Er selbst ist jedoch inzwischen ein verheirateter Vater und kümmert sich als Farmer und einziger männlicher Erwachsener hingebungsvoll um seinen Haushalt. Dieser besteht aus seiner verwitweten Mutter, seiner unverheirateten Schwester und ihren vier Kindern, sowie seiner Ehefrau und seinem einjährigen Sohn. Dieser Werdegang zeigt exemplarisch, wie Einzelne mit den Freiheiten und Möglichkeiten moderner, städtischer Lebensweisen nicht zurechtkommen und scheitern. Die Dorfgemeinschaft fängt sie mitunter jedoch wieder auf und im vertrauten dörflichen Kontext finden sie wieder Möglichkeiten einer sinnhaften Lebensgestaltung.

\section{Exkurs 3: Weibnachten 2011 - Nabuna, Koro Island}

Die erste Vorkehrung für das anstehende Weihnachtsfest stellt die Säuberung der Friedhöfe Anfang Dezember seitens der männlichen Bevölkerung dar. Der Termin wurde auf einem boca ni vanua festgelegt, geleitet vom Häuptling und dessen Sprecher, die unterschiedlichen mataqali angehören. Die Grabpflege wird mit einem Fest zur Ehrung der Ahnen abgeschlossen. Weiterhin soll die regelmäßige Dorfreinigung kurz vor Weihnachten besonders gründlich erfolgen, um das Dorf für die Festtage herauszuputzen. Da ich bereits zum 02. Dezember anreise, kann ich bei diesen Vorkehrungen mithelfen.

Nach und nach trudeln die Verwandten über die Fähren in Nabuna ein. Da jede Heimkehr sehr herzlich gefeiert wird, kommen die Ansässigen kaum mit ihren Farmarbeiten hinterher, ernten yaqona und Feldfrüchte in rauen Mengen, ohne entsprechend nachpflanzen zu können. Die sozialen Gaben, die bei den Wiedersehen ausgetauscht werden, sind dabei auch von ökonomischem Wert. Wenige Tage vor Weihnachten oder an Heilig- 
abend selbst bauen drei der fünf mataqali Nabunas eigene Zusatzunterkünfte wie in Malawai. Einer der mataqali zählt nur fünf Mitglieder im Heimatort, sodass diese für das Fest zu ihren zahlreichen Verwandten nach Suva reisen. Da der mataqali, dem ich zugehöre, dieses Jahr überraschend wenig Besucher bewirtschaftet, wird keine Zusatzunterkunft aufgestellt, sondern man trifft sich - je nach Wetterlage - auf und vor einer überdachten Veranda mit Meeresblick.

Mit einer Messe am Heiligen Abend wird das eigentliche Weihnachtsfest eingeläutet. Der Großteil der Einwohner sind Methodisten, jedoch gibt es mehrere Katholiken, die gemeinsam mit Glaubensbrüdern des Nachbardorfes Vatulele eine kleine katholische Gemeinde bilden. Diese sparen auf eine richtige Kirche, richten derzeit jedoch Privathäuser abwechselnd in Nabuna und Vatulele zu Gotteshäusern her. Während die Methodisten bis zu drei Gottesdienste pro Sonn- und Feiertag abhalten, feiern die Katholiken normalerweise nur jeweils einen. Am ersten Weihnachtstag wurde dieser in Nacamaki, dem Ort mit der größten katholischen Gemeinde und der einzigen katholischen Kirche Koro Islands abgehalten. 62 Mitglieder, damit nahezu die gesamte katholische Gemeinde, fuhren gemeinsam mit mir auf der offenen Ladefläche eines LKWs nach Nacamaki. Nachts traf sich der Großteil der Gemeinde zum gemeinsamen Feiern in Vatulele. Am Abend des zweiten Weihnachtstages stattete ich im Rahmen meines vorwiegend methodistischen mataqali einen weiteren Besuch in Vatulele ab. Nicht nur in Malawai, sondern auch in Nabuna wird die Weihnachtszeit intensiv mit der Großfamilie und der Glaubensgemeinschaft verbracht, mit zusätzlichen Besuchen benachbarter mataqali.

\subsubsection{Marktwirtschaftliche Beriehungen und Reziprozität}

Verkehrstechnisch liegt Koro Island günstig zwischen den beiden größten Inseln Viti Levu und Vanua Levu, sodass die verbindende Fähre mehrmals wöchentlich einen Zwischenstopp auf Koro Island einlegt. Die Anlegestelle liegt im Süden der Insel, Nabuna im Norden. Unter anderem aufgrund der guten Fähranbindung hat sich in Nachbarschaft zum Dorf ein kleineres Touristenressort angesiedelt. Manche Dorfbewohner können hier geringfügigen Arbeiten zumeist als Gärtner oder Haushaltshilfe nachgehen (siehe Kap. 7.3.5). Weil die Schotterstraßen auf der Insel ordentlich instand gehalten werden, gibt es mehrere Lastkraftwagen, die den fluktuierenden Waren- und Personentransport innerhalb der Insel und zum Markt nach Suva sichern. Die achtstündige Überfahrt von Suva zum Anlegesteg der Insel kostet 50FJ\$ und eine LKW-Mitnahme nach Nabuna weitere 10FJ\$. Diese Strukturen der Marktanbindung scheinen akzeptabel, zumal sich Land vergleichsweise günstig pachten lässt, sodass aus wirtschaftlichen Gründen seit den 1960ern eine Binnenmigration nach Koro stattfindet. Vorwiegend kommen Fidschianer der Lau-Inselgruppe, aber auch einzelne Rotumanen und Indo-Fidschianer haben sich niedergelassen.

Auf Fidschi wachsen die meisten Pflanzen ganzjährig und die Hauptnahrungsmittel dalo (taro,) und tavioka (manioc, cassava) brauchen etwa 7 Monate bis zur Ernte. Neben einer Subsistenzwirtschaft legen die Farmer Plantagen dieser Hauptnahrungsmittel an, um die Ernte am Markt in Suva zu verkaufen. Auch 
Kopra wird je nach Preislage verkauft. So lassen sich relativ zeitnah Gewinne erzielen, im Vergleich zum yaqona-Anbau.

Yaqona ist trotz der mehrjährigen Wachstumszeiten der größte Gewinnbringer. Ein Mann des mataqali Sawana betreibt einen yaqona-Verkaufsstand im Markt Suvas. Dieser persönliche, direkte Marktzugang wird seitens der gesamten Dorfgemeinschaft geschätzt, weshalb er mehrfach bei den Auflistungen bedeutender Einzelpersonen des Dorfes erwähnt wurde - neben einem verstorbenen Zimmerer, der in den 1970ern unentgeltlich die methodistische Kirche baute. Nicht nur der mataqali Sawana, sondern das ganze Dorf nutzt diesen persönlichen Kontakt. Man vertraut ihm und schätzt ihn, da er yaqona immer abnimmt und dies zu fairen Preisen. Auch von Nachbardörfern nimmt er yaqona an, sodass er inselweit bekannt ist. Er selbst sagt, sein Stand würde besser laufen, wenn er rein marktwirtschaftlich agieren könne, anstelle von Freundschaftspreisen und sonstigen Gefälligkeiten. Umgekehrt ehrt ihn seine soziale Funktion als Mittelsmann zwischen den Bewohnern der Insel Koro und den Angehörigen in der Hauptstadt.

Aufgrund seiner Marktkenntnisse ist er zugleich Oberhaupt der SawanaJugendgruppe. Er informiert darüber, welcher Anbau von welchen Agrarprodukten besonders lukrativ erscheint. Die Jugendgruppe übernimmt auch soziale dörfliche Verpflichtungen wie Pflege und Reinigungsarbeiten und ist auf das Wohl der Gruppenmitglieder aus. Damit ist die Gruppe nicht primär marktwirtschaftlich orientiert, weshalb die Besetzung des Leiters nicht unumstritten ist, da er seit vielen Jahren außerhalb der Dorfgemeinschaft in Suva lebt und seine Einblicke in soziale Belange begrenzt sind.

Handgeflochtene Matten werden mitunter innerhalb der Familiennetzwerke an Verwandte außerhalb des Dorfes verkauft. Ein Zugang zum Markt, um Matten gewerblich zu verkaufen, existiert jedoch nicht. Ähnlich schlecht ist der Zugang zum Fischmarkt. Lediglich die Bewohner der Siedlung Namilau haben hier einen Kontakt, mithilfe dessen sie unregelmäßig Fische verkaufen können.

Nabunas Küste ist der einzige Ort Koro Islands, wo vermehrt Landkrebse aufzufinden sind. In der fidschianischen Esskultur gilt diese Sorte Landkrebse (lairo) als Delikatesse und die Einwohner Nabunas könnten mit ihrem Verkauf vergleichsweise hohe Gewinne erzielen. sie werden jedoch ausschließlich als Gabe an Nachbargemeinden und an außerhalb der Dorfgemeinschaft lebende Verwandte verschenkt. Dadurch, dass sie nicht zum Verkauf stehen, sondern man sie ausschließlich als Geschenk erhalten kann, werden sie der Marktlogik entzogen und stellen keine Ware dar. Sie sind heilig. Landkrebse aus Nabuna geschenkt zu bekommen ist somit eine Ehre, der man sich gerne dankbar erweist, sodass ein reziproker Gabentausch innerhalb Koro Island und zu den auswärtigen Angehörigen aufrechterhalten bleibt.

Viele Dörfer Fidschis, teilweise ganze Inseln, manchmal auch einzelne mataqali behandeln einzelne Tier- oder Pflanzenarten als Totem. Diese sind nicht käuflich und profan, sondern heilig. Malawai beispielsweise sieht eine Makrelenart (tugadra) als seinen Totem an (siehe Abb. 8). Als Schwarmfisch wird er im Schnitt nur alle 
paar Jahre vor der Küste ausfindig gemacht, dann jedoch zu hunderten bis tausenden Exemplaren gefangen und zum Großteil an die Nachbargemeinden verschenkt.

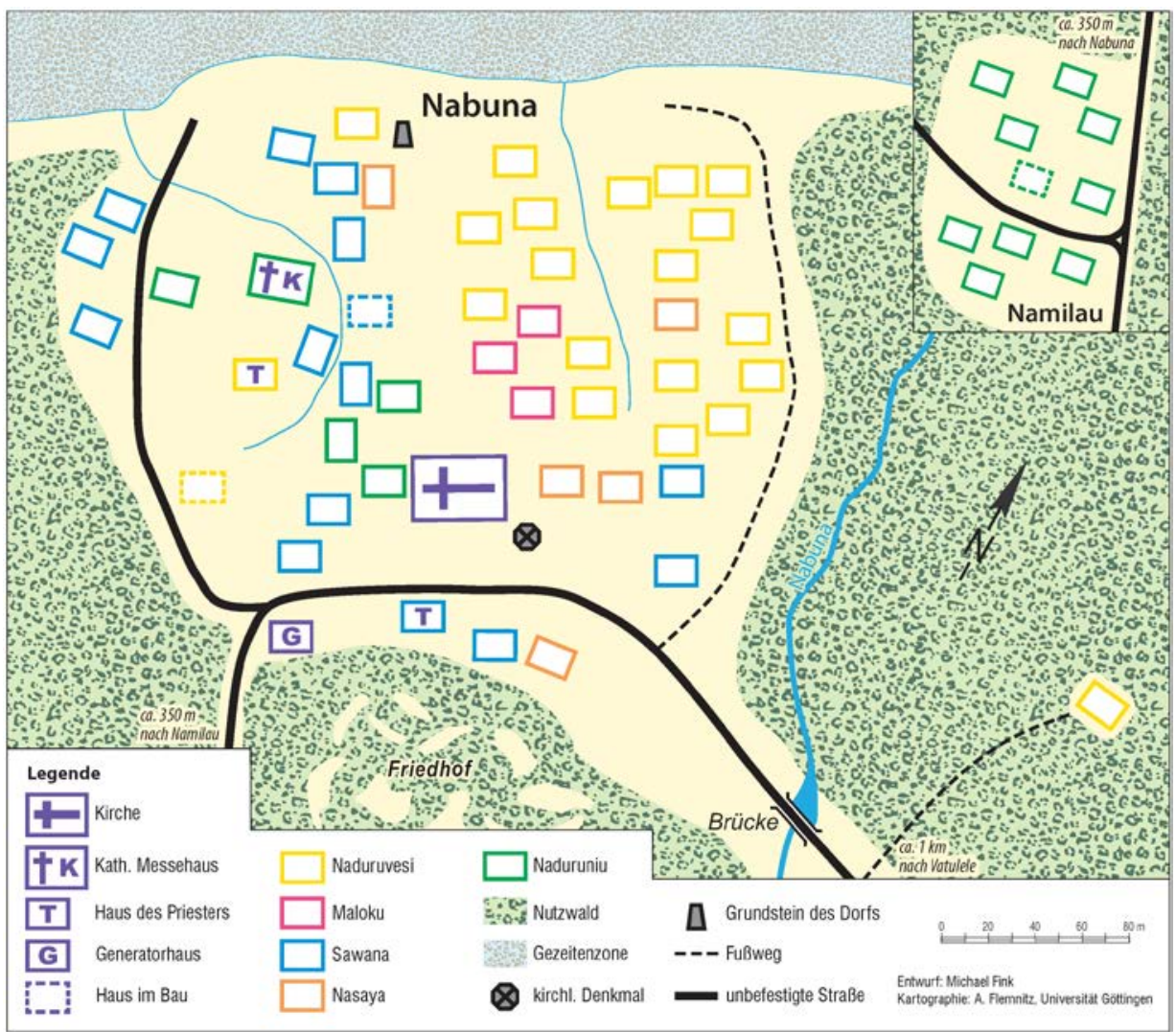

Karte 5: Nabuna

\subsubsection{Soziale Gliederung und Häuptlingswesen}

Sechs mataqali sind in Nabuna beheimatet. Der mataqali Nawavoli ist jedoch komplett fortgezogen. Während sich der Häuptlingsklan Naduruvesi aus zwei tokatoka zusammensetzt, bestehen die anderen nur aus einem. Viele Katholiken des mataqali Naduruniu haben fußläufig zum Dorf die Siedlung Namilau gegründet. Verschiedene Spannungen gaben den Anstoß zu diesem Schritt. Diese sind nicht nur religiöser Natur, sondern entspringen vielmehr sozio-kultureller Meinungsverschiedenheiten. So herrscht in der Siedlung beispielsweise keine so strikte Kleiderordnung für Frauen wie im Dorf. 


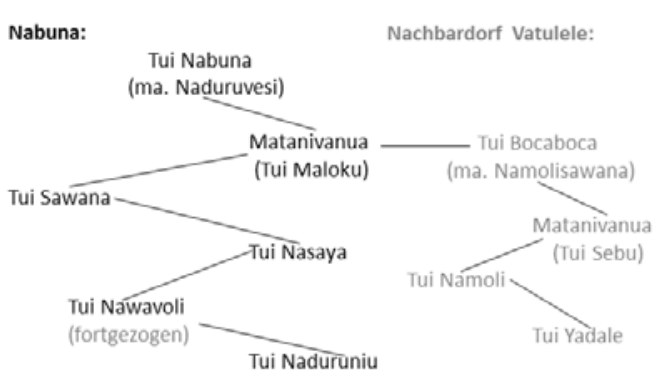

Abb. 10: Nabuna: möglicher formaler Aufbau
Während der mataqali Naduruvesi der Häuptlingsklan ist und der mataqali Maloku die Funktion des mata ni vanua innehat, ging das Wissen um die Funktionen und hierarchischen Anordnungen der weiteren mataqali verloren. Dem mata ni vanua (Tui Maloku) zufolge lässt sich die Rangfolge innerhalb Nabunas sowie zu den mataqali des Nachbardorfs Vatulele entsprechend der Abbildung 10 darstellen. Innerhalb Nabunas ist diese jedoch umstritten. Da es bei Diskussionen über die Funktionen und Hierarchien in der Vergangenheit jedoch häufig zu Handgreiflichkeiten kam, wird das Thema aktuell gemieden.

Da kein mataqali die Funktion des Häuptlingsmachers (sauturaga) innehat und sich kein alternatives System etabliert hat, wie ein Häuptling installiert wird, wird die Nachfolge weitestgehend innerhalb des Häuptlings-tokatoka entschieden. Die mangelnden Möglichkeiten einer Einflussnahme seitens der weiteren Dorfbewohner auf die Häuptlingswahl tragen zu einer mangelnden Akzeptanz des derzeitigen Amtsträgers bei. Wie auch bei Interimshäuptlingen in Malawai stellt die Person des Häuptlings in Nabuna eine Reizfigur dar. So wertschätzen viele, dass er von den 1970ern bis in die 1990er Jahre einer Lohnarbeit in der Goldmine Vatukoula auf Viti Levu nachging, da er mit den Lohneinnahmen unter anderem den Kirchenbau unterstützte. Andere hingegen werfen ihm vor, in Zeiten zentraler Veränderungen des Dorflebens nicht präsent gewesen zu sein, wichtige Entwicklungen innerhalb der Dorfgemeinschaft verpasst zu haben, und somit nur unzureichend zukunftsträchtige Entscheidungen fällen zu können. ${ }^{47}$

Mit den zentralen Veränderungen ist zum einen die Einführung der Gruppen und Komitees gemeint, in denen sich die Gemeinschaft abseits der Klanstrukturen sozialisiert. Zum anderen brachte Alkoholismus eine weitere gesellschaftliche Veränderung. Mit ihm gingen physische Gewalttaten einher, insbesondere häusliche Gewalt (mehrere informelle Gespräche und Leitfadeninterviews). Viele Dorfbewohner waren dem Alkoholismus seit den 1990er Jahren verfallen und das Dorf

\footnotetext{
${ }^{47}$ Der Tui Malawai war ebenfalls als Arbeiter auf Pazifik-weit operierenden Containerschiffen lange abwesend. Mir persönlich ist jedoch keine Kritik diesbezüglich geäußert worden. Sollte es keine größere Kritik an ihm geben, so könnte dies daran liegen, dass er noch sehr kurz im Amt ist, aufgrund persönlicher Führungsqualitäten eine hohe Akzeptanz genießt, oder dass nach Jahrzehnten ohne festen Häuptling in Malawai die Freude überwiegt, das Amt überhaupt wieder besetzen zu können.
} 
hatte inselweit einen schlechten Ruf.48 Erst als sich Mitte der 2000er die Alkoholpreise durch Steuern drastisch erhöhten und Aufklärungskampagnen griffen, bekam das Dorf diese Problematik in den Griff. Weiterhin fielen in die Zeit der Abwesenheit des derzeitigen Häuptlings die Spannungen, welche zur Abspaltung der Siedlung Namilau führten.

\subsubsection{Einbindung in traditionelle regionale Systeme}

In fidschianischen Dörfern ist jedes Haus ein geweihter Ort (siehe Kap. 7) und hat einen Namen. Es kann z.B. nach einer Pflanze oder Landschaft benannt sein, den

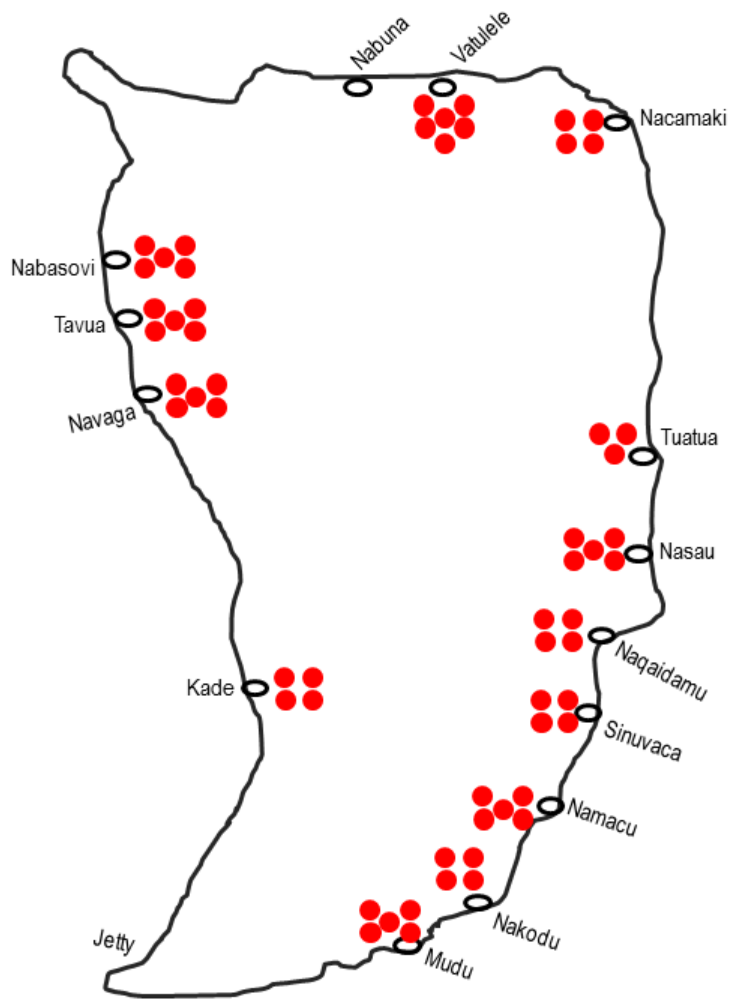

Abb. 11: Dorfberiebungen Koro Island (Entwurf: Tui Maloku) dieses Haus gewidmet ist. Ferner kann es einen Dorfoder Inselnamen tragen. In diesem Falle unterhält der Haushalt eine direkte Verbindung zum genannten Ort, welche einer BotschafterFunktion entspricht. Innerhalb Koro Islands unterhält der mataqali Naduruvesi Botschafter-Funktionen zu Kade, Nawaga und Namaca. Der mataqali Maloku steht in direkter Verbindung nach Vatulele, Naqaidamu und Nakudu. Auch Abbildung 10 zeigt, dass die Bewohner Vatuleles bei offiziellen Anliegen an Nabuna den Kommunikationsweg über den mataqali Maloku suchen sollen. ${ }^{49}$ Sawana vertritt Nacamaki, Nabasovi und Sinuvaca, Nasaya Tavua und Naduruniu Mudu. Für Außenstehende mag dieses System komplex, verwirrend oder unpraktisch erscheinen. In der

\footnotetext{
${ }^{48}$ Ein Dorfbewohner sagte, sie waren als „die PLO Koro Islands“ verschrien. Gemeint ist die Palästinensische Befreiungsorganisation PLO. Insbesondere über Militärdienste in der British Army oder als UN-Blauhelmsoldaten waren damals wie heute viele Fidschianer im Nahen Osten stationiert. Familienangehörige assoziieren mit der PLO die Gewalt im Nahen Osten.

${ }^{49}$ Nasau steht nicht in direkter Verbindung zu einem mataqali Nabunas, aber zu Sebu in Vatulele. Vertreter Nasaus können über Sebu direkt mit dem Tui Nabuna kommunizieren und müssen nicht den mataqali Maloku einbinden.
} 
Handhabung erweist es sich jedoch als äußerst praktisch, Zugänge zu verschiedensten Orten Fidschis zu haben und Anliegen dort respektvoll vortragen zu können.

Weiterhin ergeben sich über Heiratsallianzen Kontakte und Austauschmöglichkeiten zwischen den Dörfern. Zwischen Vatulele und Nabuna sind alle mataqali miteinander verwandt. Abbildung 11 zeigt die Intensität der Beziehungen Nabunas zu den weiteren Dörfern Koro Islands, die sich im Wesentlichen auf die Vernetzungen mittels Heiraten und Botschafter-Funktionen stützt.

Ein Haushalt des mataqali Nasaya unterhält eine Beziehung nach Batiki, Lomaiviti. Dieses Dorf wiederum steht in direkter Verbindung zu Bau Island. Im Zuge einer Beerdigung eines hohen Mitglieds der großen Häuptlingsfamilie aus Bau, besuchte man Batiki um gemeinsam an der Beerdigung auf Bau Island teilzunehmen. Im Zuge der mehrtägigen Trauerfeier trug man das Anliegen vor, die Jugend Koro Island solle von einer hohen Persönlichkeit Baus inspiriert werden.

Da man Angst hat, die Jugend könne Werte und Ziele in ihrem Leben verlieren, errichtete man ein diesbezügliches einwöchiges Jugendcamp, zu dem alle jugendlichen Schüler Koros eingeladen wurden. Es fand während meiner Anwesenheit in den Weihnachtsferien 2011 auf dem Schulgelände statt. Höhepunkt der Veranstaltung war ein Gottesdienst, geleitet von einem hohen Vertreter der methodistischen Kirche, welcher zugleich der Häuptlingsfamilie Bau Islands angehörte. Während seines mehrstündigen Aufenthaltes wurde er nach fidschianischem Protokoll zeremoniell beschenkt, darunter mehrere tabua, Walzähne - der höchsten Gabe im traditionellen System. Dieser enorme Aufwand in zeitlicher, logistischer und finanzieller Hinsicht zeugt von der Bedeutung der traditionellen Bände für die Bewohner Nabuna. Teil der Kubuna-Konföderation zu sein, erfüllt die Menschen mit Stolz und stellt einen zentralen Pfeiler der kulturellen Identität dar.

\subsubsection{Risiken und Chancen der Transformation}

Mit der Mobilitätssteigerung durch die Straße und Fährverbindung, mit der die Niederlassung auswärtiger Fidschianer auf Koro Island einherging, sowie den gesteigerten Arbeitsmöglichkeiten innerhalb Fidschis sowie über das Militär in Übersee erweitern sich die Aktionsradien der Einwohner Nabunas. Freiheitsgrade, wie und mit wem man seinen Alltag gestaltet, haben in beachtlichem Maße zugenommen, da man nicht mehr so einseitig wie früher innerhalb des Familienverbundes agiert, sondern sich zusätzlich in Interessengruppen zusammenfindet veranschaulicht über die formal installierten Gruppen. Ein kultureller Wandel kann also durchaus zu einer Lebensbereicherung führen.

Mit den neugewonnenen Freiheiten geht eine mangelnde soziale Kontrolle einher. Westliche Drogen und Alkoholgenuss - bzw. zumindest die Möglichkeit des Massenkonsums - waren gesellschaftlich unbekannt und daher das Wissen um gefährliche Dimensionen nicht gesellschaftlich verankert. Das Trinken von Alkohol war kulturell nicht eingebettet, sodass es mangels sozialer, kulturell tradierter 
und damit weitläufig akzeptierter Kontrollmechanismen zerstörerische Auswüchse annahm. Das traditionelle Wissen reichte nicht aus, die neumodischen Phänomene zu handhaben. Das Erlernen über einen gesellschaftlich adäquaten Umgang mit neuen Möglichkeiten der Lebensgestaltung benötigt demnach Zeit und ist in der Übergangsphase mit hohen Risiken verbunden.

Die Auswirkungen des Transformationsprozesses lassen sich als ambivalent charakterisieren. Mit dem Wandel geht Wissen um traditionelle Funktionen verloren, wie der unklare formale Aufbau des Dorfes und die Häuptlingsfrage zeigen. Es gibt Ängste, insbesondere die Jugend könne ihre kulturelle Identität verlieren und damit einhergehende Werte, aus denen sich ein Lebenssinn speist. Man entwickelt jedoch neo-traditionelle Gegenmaßnahmen, das Wertefundament zu festigen. Mittels erweiterter Handlungsmöglichkeiten in finanzieller wie logistischer Hinsicht nutzt man also einerseits aktiv Aspekte des gesellschaftlichen Wandels und reproduziert bzw. beschleunigt diesen - um andererseits andere Aspekte des Transformationsprozesses zu steuern bzw. zu bremsen.

\subsection{Dravuni: Sozial-ökonomische Einblicke}

Dravuni liegt an der Ostküste Viti Levus in der Provinz Tailevu an der Flussmündung des Nukurua. Aktuell leben in der Dorfgemeinschaft vier tokatoka die sich zu zwei mataqali zusammenschließen. Die vier tokatoka Dravunis haben als Funktionen sauturaga, Häuptlingsmacher, mata ni vanua, Sprecher des Häuptlings, bete, Priester bzw. Diener und gonedau, Fischer. Es gibt keinen Häuptling, da der fünfte tokatoka, welcher den Häuptling stellt, vollständig ausgewandert ist. Ob bzw. wann Mitglieder des Häuptlings-tokatoka zurückkehren und wie man ggfs. langfristig mit dem Problem umgehen soll, ist offen. Nach Jahren, in denen das Amt gänzlich unbesetzt blieb, behilft man sich nun mit einer Interimslösung. Mir wurde auf Englisch rezitiert, mit welchen Worten man den Ältesten des bete-tokatoka in seine neue Aufgabe lobte: „You have served the village for 80 years. Now, please serve the village as chief." Mir wurde suggeriert, dass hier sprachlich eine dem Anliegen entsprechend exzellente Formulierung geglückt sei, indem man einerseits die familiär angestammte Position als bete herausstreicht und damit indirekt den Interimscharakter als Häuptling verdeutlicht. Andererseits werden die persönliche Lebensleistung herausgestellt, die ihn zu seiner Aufgabe befähigt, sowie der Respekt und die Dankbarkeit betont, die einem Häuptling für seine schwere Aufgabe zu Teil werden sollte.

In Dravuni wohnt zudem ein Freiwilliger des US-amerikanischen Peace Corps. $\mathrm{Ihm}$ wurde ein bure gebaut, ein Haus nach traditioneller Baukunst. ${ }^{50} \mathrm{Im}$ Sinne des

\footnotetext{
${ }^{50}$ Ansonsten stellt ein bure in keiner Gemeinde, die ich besucht hatte, ein Wohnhaus dar. Wellblech, Zement und Schnittholz sind die gängigen Baumaterialien. Das bure hingegen besteht aus Lehmböden, Stämmen und Ästen, sowie geflochtenen Blättern und Gräsern. Die Dächer sind steil und die Fenster klein, sodass es kaum windanfällig ist. Anstelle von Nägeln knotet man die Elemente zusammen, sodass das Haus elastisch ist. Somit ist es recht widerstandsfähig gegenüber Zyklonen.
} 
Friedenscorps wird der Freiwillige alle zwei Jahre ausgetauscht. Während meiner Anwesenheit war der zweite Freiwillige vor Ort. Die Aufnahme stellt ein hohes Gut in Fidschi dar und viele Gemeinden bewerben sich beim Peace Corps. Finanziell stellt eine solche Aufnahme keinen Gewinn für die Gemeinschaften bereit. Stattdessen freut sich die Dorfgemeinschaft an der Abwechslung, die er in die Gemeinde bringt und der Möglichkeit, ihre Gastfreundschaft ausleben zu können. Zudem erfährt das Dorf von anderen fidschianischen Gemeinden viel Anerkennung. Erzählte ich andernorts Fidschianern von Dravuni, so war der Peace Corps oftmals die einzige Assoziation, die man mit dem kleinen Dorf hatte.

\section{Exkurs 4: Weihnachten 2012 - Dravuni, Tailevu, Viti Levu}

Dravuni ist ein winziges Küstendorf an der Ostseite der Hauptinsel Viti Levu, nur etwa eine Autostunde von der Hauptstadt entfernt. Busse bringen einen fast stündlich für unter 5FJ\$ zur entsprechenden Abzweigung der größten Landstraße Fidschis, 20 Fußminuten vom Dorf entfernt; ein Taxi ins Dorf kostet etwa 35FJ\$. Ich reise am 28. November an.

Auf einem boca ni vanua, geleitet von einem Interimshäupting und weiteren Dorfältesten, in Vertretung für die komplett ausgewanderte Häuptlingsfamilie, wurde im November die vorweihnachtliche Grabpflege diskutiert. Entgegen bisheriger Praxis sah es die Jugend nicht ein, mühselig nur mit Macheten oder bloßen Händen den Friedhof zu reinigen. Stattdessen wollte man zuvor mit Spritzmitteln Unkraut vernichten und sich am Tag selbst mit elektrischen Gestrüppschneidern die Arbeit erleichtern. So könnte man mehr Zeit und Mühen in die Herrichtung des großen Festes zu Ehren der Ahnen investieren. Die Ältesten sahen dies jedoch als eine Abkehr von wichtigen Traditionen, die Unheil hervorbringen würde. Und sollten die Gräber nicht entsprechend traditioneller Ordnung gepflegt werden, so wäre es verlogen, der Arbeit mit einem Fest zu huldigen.

Im Ergebnis wurden nicht-traditionelle Hilfsmittel eingesetzt und das Fest viel aus. Lediglich vor- und nach der Reinigung wurde eine kurze yaqona-Zeremonie abgehalten, um den geweihten Ort für die Arbeiten unbedenklich betreten zu können. In Einzelgesprächen zeigten beide Lager Verständnis für die jeweils andere Sichtweise, beklagten jedoch mangelnde Einsicht beim Gegenüber. Da christliche Nächstenliebe und Vergebung hohe Tugenden sind, verhält man sich nicht nachtragend und die Reibung belastete nicht das eigentliche Weihnachtsfest.

In den Tagen vor und während des Weihnachtsfestes kamen die andernorts lebenden Verwandten in Scharen zurück zur Heimat. Auch zwei Soldaten, die über die britische Armee in Übersee stationiert waren, konnten über Weihnachten zurückkehren. Ein Haushalt wuchs von 3 auf 14 Personen an, ein anderer Haushalt von üblicherweise 8 Personen stieg auf 34. Insgesamt wurde die übliche Einwohnerzahl des 19 Haushalte zählenden Dorfes mehr als verdoppelt.

Gemeinhin wird es als gemütlicher im Vergleich zu Standart-Häusern wahrgenommen. Die Gemeinde Lekanai auf Gau Island hat daher ihr Gemeindehaus als bure errichtet. Vereinzelte Haushalte der Gemeinden, die ich besuchte, daben zudem kleine bure als Zweithaus bzw. Aufenthaltsraum. 
Dravuni besteht aus zwei mataqali, und Weihnachten wurde entsprechend in den beiden Klans verbracht. Der eine nutzte als Raum für die Festivitäten die Gemeindehalle, der andere einen großen geliehenen Pavillon. Wie in den anderen Dörfern wurden die Weihnachtstage nicht nur intensiv im geschlossenen Klanverbund zelebriert, genauso besuchte man darüber hinaus Bekannte in Nachbardörfern und tauschte Gaben aus. Das Essen in Dravuni war zur Weihnachtszeit besonders divers, da nicht nur auf eigene Farmprodukte zurückgegriffen werden konnte, sondern zusätzlich aufgrund der Stadtnähe und des Anschlusses des Dorfes ans Stromnetz leicht verderbliche und zu kühlende Lebensmittel wie Tiefkühlhähnchen und Speiseeis die Mahlzeiten bereicherten. Aufgrund eines Zyklons war das Dorf jedoch vom 18. bis 23. Dezember vom Stromnetz abgeschnitten, sodass die Essenseinkäufe kurzfristiger getätigt wurden und im Vergleich zu vorherigen Jahren wohl etwas spärlicher ausfielen.

Das Dorf besteht aus zwei christlichen Glaubenskongregationen. Die Seventh Day Adventists hielten ihre Weihnachtsmessen in ihrer Gemeindekirche im Nachbardorf Naimasimasi ab. Der Großteil jedoch sind Methodisten, die ihre Kirche im Dorf haben. Aufgrund der kleinen Mitgliederanzahl und Unstimmigkeiten, die Kirche würde zu viel Zeit und die Kollekten zu viel Geld berauben, beschränkt man sich auf einen Gottesdienst pro Feiertag anstelle der üblichen bis zu drei Gottesdiensten anderer Methodistengemeinden.

\subsubsection{Einbindung in politische, traditionelle und kirchliche Gliederungssysteme}

Gemeinsam mit Naisaumua und Naiborebore bildet Dravuni den Tai Distrikt. Die Dörfer dieses Distrikts haben eine traditionelle Verbindung. Historisch gesehen kam Tai im 19. Jahrhundert eine wichtige Rolle zu. Im Zuge der Nationalstaatsbildung waren sie gefürchtete Krieger, die zunächst für die Herrscherfamilie mit Sitz im benachbarten Verata gegen Bau kämpften (Burns 1963: 67). Mitte des 19. Jahrhunderts jedoch wechselten sie die Seiten und trugen dazu bei, für Cakobau die Vormachtstellung innerhalb Fidschis zu erobern. Viele dieser Krieger und deren Nachfahren ließen sich in den eroberten Gebieten nieder, vor allem im Landesinneren Viti Levus und in der Lomaiviti-Inselgruppe. Im Jahr 2000 wurde in Dravuni ein Treffen für die Nachfahren ausgerichtet. So bestehen in Dravuni noch lose Kontakte zur Großfamilie in Malawai, Gau Island, deren Oberhaupt dort Häuptlingsansprüche meldet (siehe Kap. 6.2.2).

Die der Bucht vorgelagerte Insel Viwa war zudem - nach der Missionierung der Insel Lakeba ab 1835 - die zweite Insel, die christianisiert wurde. Dort wurde die Bibel erstmals ins Fidschianische übersetzt und von hier aus wurde gesamt Viti Levu konvertiert. Dravuni behauptet von sich, das erste christliche Dorf der Hauptinsel zu sein und einer der ursprünglichen Missionare liegt hier beerdigt.

Die Mitglieder eines tokatoka sind überwiegend Seventh Day Adventists. Ihre Kirche steht im Nachbardorf Naimasimasi. Die meisten Dorfbewohner sind jedoch Methodisten. Sie haben eine eigene Kirche im Dorf, die vom vakataua geleitet wird. Der talatala sitzt auf Viwa. Mehrmals monatlich stehen gemeinsame Treffen an, zumeist in Viwa, aber auch in den weiteren Gemeinden des kirchlichen 
Bezirks, welche ebenfalls nur per Boot erreicht werden können. Derzeit hat die Gemeinschaft Dravunis kein eigenes Boot, sodass sie für die Überfahrten ein Boot einer benachbarten Gemeinde chartern müssen. Die Finanzierung stellt ein großes Problem für die Kirchenmitglieder dar. Anträge an die methodistische Kirche, ihnen ein eigenes Boot zur Verfügung zu stellen, scheiterten bisher. Weiterhin überlegen Einzelne daher, ob man aus dem Bezirk austreten könne und sich dem Bezirk anschließen könne, denen die weiteren Dörfer des Tai Distrikts angehören.

Dieses Beispiel verdeutlicht das komplexe institutionelle, räumlich inkonsistente Geflecht, in dem sich fidschianische Dorfbewohner bewegen. In Dravuni stimmt ausnahmsweise die staatliche Verwaltungsebene Tai Distrikt mit einem historischen Stamm überein. Der lokale methodistische Zusammenschluss hingegen ist historisch unabhängig von Stammesverbindungen gewachsen. Die Gemeinschaft der Seventh Day Adventists bildet wiederum ein drittes sozialräumliches Gebilde. Naturräumlich wiederum liegt Dravuni an der Flussmündung des Nukurua. Die größte Holzplantage Fidschis liegt am Oberlauf. Die „Nukurua Forest Reserve" erstreckt sich über drei Provinzen und schießt Ländereien vieler Dorfgemeinschaften ein, nicht jedoch solche des Tai Distrikts. Die MahagoniAbholzung wird für vermehrte Sedimentation und - damit einhergehend - für ökologische Schäden wie vermehrte Dorfüberflutungen und rückläufige Fischbestände im Fluss und der Meeresbucht mitverantwortlich gemacht (ProblemScorings, Venn-Diagramme und Einzelgespräche). Es existiert keine soziokulturelle Verbundenheit mit dem halbstaatlichen Wirtschaftsunternehmen und über die staatliche Verwaltungskommunikationsstruktur zwischen dem turaga ni koro und dem mata ni tikina (Distrikt-Verwalter) können bisher keine Zuständigkeiten herausgefunden werden. Ein Kommunikationsweg, über den der Konflikt mit Verantwortlichen diskutiert und Lösungen generiert werden können, ist somit derzeit nicht gefunden.

Potenziell verbinden die staatlichen und kirchlichen Gliederungseinheiten Gemeinden direkter miteinander, als bloße Bezüge über das Häuptlingssystem, sodass die Handlungsmöglichkeiten prinzipiell erweitert werden. In der Praxis überwiegen jedoch negative Begleiterscheinungen. Schwierigkeiten entstehen, wenn sich aufgrund ungeklärter Zustände mehrere oder aber keine Personen zuständig bzw. verantwortlich sehen, was auch zu doppelter bis dreifacher Bürokratie führen kann. Unübersichtlichkeiten und Unklarheiten über Handlungsmöglichkeiten führen zu Unsicherheiten. Dies verwässert Verwirklichungschancen. Zufälle bzw. als extern wahrgenommene, nicht zu durchschauende Umstände entscheiden über den Erfolg oder Misserfolg von Handlungen und spalten die Gesellschaft auf moralisch nicht nachzuvollziehende Weise in Gewinner und Verlierer. Alle von mir besuchten Gemeinschaften preisen daher das Häuptlingssystem, da es in ihren Augen klar und verständlich strukturiert und legitimiert ist. 


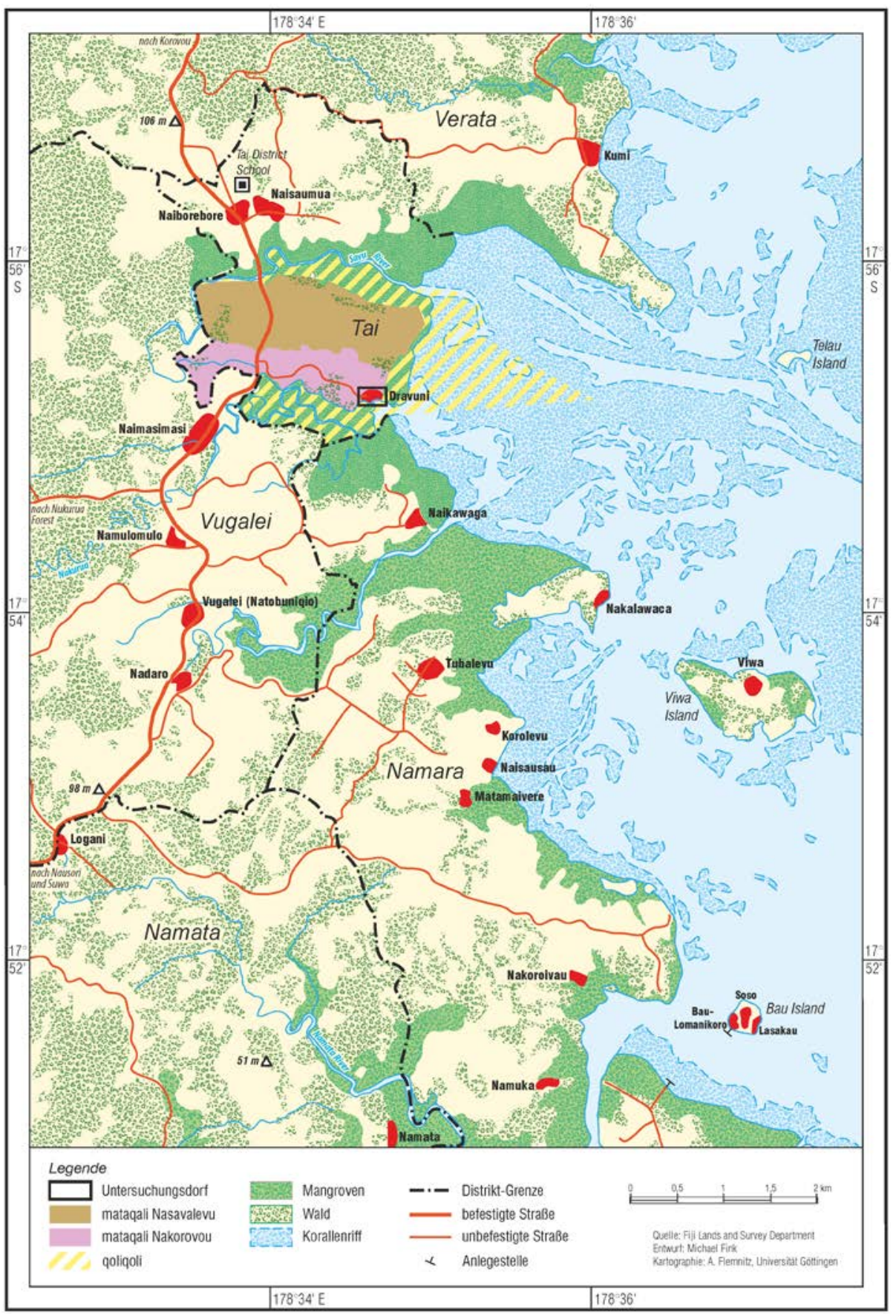

Karte 6: Umland Tai Distrikt 


\subsubsection{Marktwirtschaftliche Beriehungen und lokale Entwicklungsprojekte}

Das Dorf besteht aus 19 Häusern, mehrere sind nur von jeweils einer Person bewohnt. Insgesamt wohnen ca. 60 Personen dauerhaft in Dravuni. Die Hauptstadt Suva, wo viele Verwandte leben, liegt nur ca. 1 Stunde mit dem Auto entfernt. Die Bewohner erhalten daher häufig Besuch. Der Großteil der Ortsansässigen sind Kleinkinder und Schüler, umgekehrt sind viele Einwohner älter als 70 Jahre. Von den wenigen Einwohnern im erwerbsfähigen Alter pendeln manche zwecks Beruf oder Weiterbildung. Viele der Älteren sind zuvor einer Lohnarbeit nachgegangen und haben dafür zumeist auswärts gelebt, manche sind auch gependelt. Mit dem Renteneintritt ließen sie sich dann permanent im Dorf nieder. Ein Dorfbewohner arbeitete und wohnte jahrelang als Bankangestellter in Suva. Die Zwänge der Lohnarbeit und die Versuchungen des städtischen Lebens ließen ihn jedoch nicht glücklich werden, sodass er Mitte dreißig bewusst zurück ins Dorf zog. Über seine Aufgaben, Funktionen und Pflichten für die Gemeinschaft sieht er als Farmer bessere Möglichkeiten, sich selbst zu verwirklichen, als dies im städtischen Kontext möglich sei.



Karte 7: Dravuni

Seitens der Dorfbewohner bestehen keine persönlichen Kontakte zu Standbesitzern in den Märkten der Städte. Bringt man Agrarprodukte zum Markt, will man nicht auf ihnen sitzen bleiben. Die Standbesitzer sind daher in der besseren Verhandlungsposition und drücken die Preise. Diesem System setzen sich die 
Dorfbewohner nur sporadisch aus. Landwirtschaft dient im Wesentlichen der Subsistenzversorgung und dem reziproken Gabentausch innerhalb der Familiennetzwerke. Rücküberweisungen sichern die Lebenshaltungskosten. Nichtsdestotrotz sehen die Dorfbewohner den erschwerten Marktzugang als eines der größten Herausforderungen ihrer Lebensgestaltung an (Venn-Diagramme und ProblemScorings).

Die nicht pendelnden Erwachsenen bilden den präsentesten und aktivsten Kern der Dorfgemeinschaft. Er besteht aus nur einer Handvoll Männer und Frauen. Sie erledigen den Großteil der Gemeinschaftsarbeit des Dorfes wie Reinigungs- und Reparaturarbeiten oder Landschaftspflege. Darüber hinaus betreiben sie weitere Projekte, um das Dorf voranzubringen.

Um finanzielle Unterstützung seitens staatlicher Institutionen zu erhalten, treten die Akteure in wechselnder Besetzung in verschiedensten Komitees auf. So ist es ein zentrales Anliegen, für die Gemeindehalle sanitäre Anlagen zu installieren und eine gemeinsame Kochstelle zu errichten, da bisher Einrichtungen einzelner privater Haushalte herhalten. Einen diesbezüglichen Antrag stellten die Frauen als Frauengruppe beim Frauenministerium. Diese erhielt als Auflage, zunächst die Gemeindehalle zu vergrößern, damit sich die Investition lohne. Als Entwicklungskomitee stellten die männlichen Farmer daraufhin einen Antrag beim Ministerium für ländliche Entwicklung zur finanziellen Unterstützung bei der Erweiterung der Gemeindehalle. Diesem wurde stattgegeben, dass zwei Drittel der Kosten übernommen würden.

Parallel treten diejenigen, die Kinder im schulpflichtigen Alter haben, als Schulkomitee auf. Für die Tai Grundschule, welche gemeinsam mit Naisaumua und Naiborebore betrieben wird, traten die Vertreter der drei Dörfer zwecks der Sanierung eines Schulgebäudes in Kontakt mit dem Schulministerium. Auch hier wurde eine finanzielle Unterstützung zugesichert. Die männlichen Farmer der Dorfgemeinschaften bilden Landwirtschaftskomitees, welche in einem losen Bündnis zueinander stehend beim Landwirtschaftsministerium einen Gemeinschaftsantrag zur Bewirtschaftung je eines Feldes für die jeweilige Dorfgemeinschaft stellten, um die Startfinanzierung des Schulprojektes - sowie in Dravuni für die Gemeindehalle - zu erwirtschaften. Das Landwirtschaftsministerium gab dem Antrag statt und verteilte kostenlos Werkzeug und Saatgut, hielt einen workshop zum Anlegen von Ingwer-Plantagen und stellte einen Kontakt zu einem international agierenden Abnehmer her. Weiterhin organisierten sich die Dorfgemeinschaften in Jugendgruppen, welche in Dravuni personell stark mit den anderen Komitees überlappte. So formiert veranstaltete man mit Unterstützung des Ministry of Youth ein Rugby-Turnier zwecks Fundraising zum Schulbau.

Gleichzeitig pflanzen die Dorfbewohner Bäume für ein „One Million Trees“Projekt des Ministry of Forestry. Die Gemeinschaft züchtet und verkauft Jungpflanzen, welche zur Wiederaufforstung in waldfreien Gebieten Fidschis angepflanzt werden. Den Kontakt hierzu konnte ein Pendler herstellen, der beim Ministry of Forestry arbeitet, welches in diversen Verbindungen zur UN und zu verschiedenen 
weiteren Entwicklungsorganisationen steht, die das Projekt aufziehen. Dieses Projekt wird im Wesentlichen im Rahmen der methodistischen Kirchengruppe aufgezogen, sodass die Einnahmen zu je einem Drittel für die Kirche, die Schule und die Gemeindehalle verwendet werden sollen.

\subsubsection{Gruppenbildung aus politisch-administrativen Zwängen}

Dass die Dorfgemeinschaft formelle Gruppen mit Vorsitzenden, Schatzmeistern und Schriftführern bilden, entsteht weitestgehend nicht aus intrinsischem Interesse. Sie stellen eine Bedingung dar, um finanzielle Unterstützung seitens staatlicher Ministerien zu bekommen. Ein Mehrwert für das Sozialgefüge, formell eine Gruppe zu sein und Protokoll zu halten, erschließt sich nicht. Die Gruppenbildung wird als Auflage zur Kommunikation mit staatlichen Vertretern gesehen. Auch weil die Kommunikation nicht direkt zwischen den Gruppenvorständen und den Regierungsangestellten stattfindet, sondern über den turaga ni koro läuft, bleiben die staatlichen Institutionen meist abstrakt und gesichtslos. ${ }^{51}$ Gerade in einem kleinen Dorf wie Dravuni sind Einzelpersonen parallel im Jugend-, Schul-, Elektrizitäts-, Gesundheits-, und je nach Geschlecht im Frauen-, oder Landwirtschafts- und Entwicklungskomitee aktiv und haben drei bis vier Ämter zugleich inne - zusätzlich zu ihren Funktionen im Häuptlingswesen oder kirchlichen Gruppierungen. Der Sinn dieses bürokratischen Aufwandes erschließt sich den Dorfbewohnern nicht, sondern wird als Zwang empfunden, dem man in der Hoffnung auf staatliche Förderungen nachgibt.

In der Alltagspraxis weiß man bei der Feldarbeit auf der Ingwer-Plantage nicht, ob man dies gerade als Jugend-, Frauen-, Landwirtschafts-, Schul- oder Entwicklungsgruppe tut. Identifikationen über die Gruppenzugehörigkeit bleiben aus, auch wenn man zeitlich betrachtet 40 und mehr Stunden die Woche in solchen Gruppen verbringt (Ergebnisse des Fragebogens, siehe Anhang). Familie, Kirche und Häuptlingswesen bleiben die identitätsstiftenden Rahmen, die die Dorfgemeinschaft zusammenhält. Die Dorfbewohner stehen in enger sozialer Verbindung zueinander und haben einen starken Gemeinschaftssinn, die Zugehörigkeit zu formellen Gruppen bestärkt jedoch nicht die Identifikation.

Das folgende Zitat einer Village Nurse ${ }^{52}$ bei einem boca ni koro verdeutlicht die Müßigkeit im Umgang mit als Parallelstrukturen wahrgenommener staatlicher Institutionen: „Das Gesundheitsministerium wird uns demnächst einen unangekündigten Besuch abstatten. Solange sollten wir Sachen, die gesundheitsschädlich

\footnotetext{
51 Gerade die Mitglieder der Frauengruppe empfinden den Kommunikationsweg mit dem Frauenministerium über den männlichen turaga ni koro als unnötig kompliziert bis paradox.

52 Als Teil der staatlichen Basis-Gesundheitsfürsorge wird aus jedem Dorf eine Person in einem einbis mehrwöchigen workshop in Grundlagen medizinischer Versorgung wie Erste Hilfe ausgebildet. In den meisten Dörfern existiert parallel hierzu ein traditioneller Heiler bzw. eine Heilerin. Erst mit der Militärregierung unter Bainimarama ist man dazu übergegangen, die angestammten, auch aktuell noch von den Dorfgemeinschaften akzeptierten Ansprechpersonen für Gesundheitsfragen und deren Wissen anzuerkennen und speziell diese auszubilden, sofern diese es wünschen.
} 
sind, unterlassen." Da es keiner Aufklärung bedurfte, was „gesundheitsschädlich“ sei, ist demnach seitens des Gesundheitsministeriums bereits Wissen über vermeintlich gesundheitsschädigendes Verhalten in die Gemeinschaft getragen worden. Es findet jedoch im Alltag keine Anwendung. Empowerment findet nicht statt, da Eigentümerschaft ausbleibt. Das vorhandene Wissen wird nicht ins Alltagshandeln inkorporiert; ist nur von praktischer Relevanz, sofern es um das Vermeiden von Stress mit staatlichen Institutionen geht. Aufgrund der fehlenden Akzeptanz des Staatsapparates verpufft ein möglicher Mehrwert.

Staatliche wie nichtstaatliche Institutionen werden als externe, abstrakte Gebilde wahrgenommen. Die Trennung wird verschärft, wenn die auswärtigen Akteure aus Ignoranz oder vermeintlicher Effizienz auf das traditionelle Protokoll verzichten, zu dem ein sevusevu als Willkommenszeremonie gehört. Als während meiner Anwesenheit im Zuge eines Zyklons die Stromleitung ausfiel, kamen mehrfach Elektriker ins Dorf, um die Schäden zu begutachten. Da sie optisch klar als Elektrotechniker erkennbar waren und der Sinn ihrer Anwesenheit aufgrund des Stromausfalls keinerlei Erklärung bedurfte, ließ man sie gewähren. Da sie sich jedoch nicht offiziell vorgestellt hatten, blieben sie unbekannt und wurden als potentielle Gefahr und damit als ein erheblicher Störfaktor im Dorf wahrgenommen. Zusammenarbeiten fußen in Fidschi auf persönliche Beziehungen. Werden diese aufgrund eines mangelnden sozio-kulturellen Gespürs seitens externer Akteure nicht adäquat aufgebaut, bleiben ihre Möglichkeiten begrenzt, ihre Anliegen umzusetzen.

\subsection{Sozialräumliche Dimensionen von Familiennetzwerken}

Die Intensität des sozialen Miteinanders innnerhalb fidschianischer Dorfgemeinschaften ist herausragend. Für Austausch, Vergnügen, Kooperation und gegenseitige Hilfe bieten die vielen sozialen Institutionen, deren Teil man ist, erfolgreiche Plattformen. Auf Basis der traditionellen Abstammung und des Häuptlingswesens bieten die Großfamilie, der Klan, die Dorfgemeinschaft und die Stammeszugehörigkeit Zusammenschlüsse. Darüber hinaus trägt der Rahmen kirchlicher Gemeinschaften zur Befähigung einer individuellen Selbstverwirklichung bei. Weiterhin bergen die sozialen wie ökonomischen Interessengruppen als Alternativen zum Handeln in familiären und religiösen Strukturen Potentiale zur Selbstbestimmung. Aufgrund ihrer formalen Anerkennung staatlicherseits als Vertretung der Zivilgesellschaft könnten sie zur Durchsetzung politischer Ziele beitragen. Weil der Staatsapparat jedoch weitestgehend als ein künstliches, kulturell unvertrautes Gebilde wahrgenommen wird, bleiben Identifikationen aus und Potentiale politischer Dimensionen einer Selbstverwirklichung werden wenig genutzt.

Gerade auch die Vielschichtigkeit der Organisationsformen reduziert die latenten Gefahren, die in sozialen Beziehungen stecken. Mannigfaltige Kooperationen zur Selbstverwirklichung und Steigerung der Lebensqualität schaffen soziale Sicherheit, da einseitigen Abhängigkeiten entgegengewirkt wird, welche zu Zwän- 
gen, Gewalt und Unterdrückung verleiten könnten. Als besondere Stärke sind fidschianische Dorfbewohner nicht nur lokal in ihren Gemeinschaften verankert, sondern unterhalten Netzwerke, welche zum Teil globale Dimensionen erlangen. Punktuelle Einblicke in die familiären Beziehungsgeflechte dreier Einzelpersonen (siehe Karte 8) möchte ich nun genauer vorstellen.

\section{Exkurs 5: Drei Familiennetzwerke}

Ana ${ }^{53}, 76$, Dravuni. Interview am 10.12.2012

Ana ist 76 Jahre alt und zog 1955 mit ihrem Ehemann nach Dravuni. Dieser war von 1959 bis 2003 der vakatana der methodistischen Dorfgemeinde. Er verstarb 2008 im Alter von 82 Jahren. Ihre Mutter stammt aus Somosomo, Gau Island. Heute hat sie keinen Kontakt mehr dorthin. Ihr Vater stammt von der Insel Ono, Kadavu, wo auch Ana aufwuchs. Bis heute hält sie Kontakt zu ihrem Heimatort und wird das diesjährige Weihnachten dort verbringen, wo sie insbesondere von ihren Neffen freudig erwartet wird. Letztes Weihnachten kamen umgekehrt viele Verwandte aus Ono nach Dravuni.

Sie hat acht Söhne und drei Töchter und sagt voller Stolz, dass sie Schwiegertöchter aus allen Konföderationen Fidschis hat. Ein Sohn, zwei Schwiegertöchter und mehrere Enkel leben mit ihr in Dravuni. Ihr ältester Sohn lebt in Serua auf Viti Levu. Er schaut regelmäßig vorbei und unterstützt die Familie. Sein ältester Sohn lebt in Vanuatu und Ana hält per Telefon intensiven Kontakt zu ihrem Enkel. Zwei Söhne wohnen in Suva und aufgrund der Nähe kann man sich häufig besuchen und gegenseitig aushelfen. Ein weiterer Sohn ist seit zehn Jahren über das britische Militär im Ausland und lebt derzeit in England. Auch zu ihm konnte über all die Jahre ein intensiver Kontakt aufrecht erhalten bleiben, zumal er in dieser Zeit mehrfach für einen Heimaturlaub zurückkehrte. Lediglich zu einer Tochter, die auf Taveuni lebt, ist der Kontakt auf unregelmäßige Telefonanrufe reduziert.

Tobe, 36, Nabuna. Interview am 14.12.2011

Tobe ist 36 Jahre alt und wuchs in der Siedlung Nagadro auf Koro Island auf. Seine Familie stammt aus Moce, eine Insel der Lau-Gruppe und sein Großvater kam als Straßenarbeiter in den 1960ern nach Koro. Er selbst hat seine Herkunftsinsel nie besucht. Tobe wuchs auf Koro auf, lebte lange in Suva und kam vor wenigen Jahren für einen Weihnachtsurlaub nach Koro zurück - und ließ sich nieder. Als sein Großvater verstarb, zogen fast alle Verwandten nach Suva. Tobe blieb und übernahm die Landpacht. Koro stellt seine Heimat dar. Er heiratete eine Frau des mataqali Maloku in Nabuna und lebt mit ihr und einer einjährigen gemeinsamen Tochter im Dorf. Er hat viele leibliche Verwandte in Suva, besucht sie jedoch selten, da er sich im Dorf wohler fühlt. Auch zu seiner auswärts lebenden, angeheirateten Verwandtschaft pflegt er keine intensiven Beziehungen. Während die wenigen in Nabuna wohnenden Familienmitglieder des mataqali Maloku - neben seiner Frau und Kind noch ihr Onkel und ihre Tante 2. Grades - Weihnachten bei ihrer Verwandtschaft in Suva feierten, blieb er im Dorf zurück. Er feierte stattdessen mit Freunden in Nagadro, Nabuna und Vatulele. Er bewegt sich bewusst mehr in seinen kirchlichen Netzwerken und dörflichen Gruppen, denn in Verwandtschaftsnetzwerken.

${ }^{53}$ Die Namen sind anonymisiert. 




Karte 8: Drei Familiennetrwerk-Kartierungen 
Tulia, 42, Nabuna. Interview am 09.12.2011

Tulia ist 42 Jahre und hat in den mataqali Naduruniu in Nabuna eingeheiratet. Ursprünglich kommt sie aus Namacu, einem anderen Dorf auf Koro Island. In ihrer Erzählung, wo sie welche Verwandten und Bekannten hat, beschränkt sie sich ausschließlich auf lineare Verwandte, auf die angeheiratete Verwandschaft des tokatoka Naduruniu geht sie im Rahmen ihrer Prioritätensetzung aus Zeitgründen in diesem Gespräch nicht ein.

Besonders enge Kontakte pflegt sie zu Geschwistern und Cousins und Cousinen, welche in Suva, in Sigatoka oder aber anderen Dörfern Koro Islands leben. Ein kleiner Bruder, zu dem sie ebenfalls eine enge Bindung aufrecht erhält, hat ein Lehramtsstudium abgeschlossen und arbeitet seit mehreren Jahren in einem internationalen Forschungsprojekt auf Kia, nördlich von Vanua Levu. Regelmäßigen Kontakt hält sie auch zu mehreren Verwandten in Australien und einem in Neuseeland. Ein enger Verwandter ist über das britische Militär in England stationiert und aktuell hat man regen Kontakt. Weitere Verwandte, zu denen Sie jedoch mitunter nur über dritte den Kontakt aufrecht erhält, leben unter anderem in Nadi, Savusavu oder in Levuka auf Ovalau, auf Vanua Levu, Vanua Balavu, Cicia und Taveuni. Bei Bedarf könnten hier problemlos und schnell wieder enge Bände geknüpft werden. Zu einer Schwester, die in einem Nachbardorf auf Koro lebt, ist der Kontakt abgebrochen.

Räumliche Distanzen haben nur bedingt Einfluss auf die Intensität sozialer Beziehungen. Dank moderner Kommunikationsmedien können jegliche Entfernungen überwunden werden. Auf den entlegenen Inseln hat man nur begrenzten Zugang zu Strom, Generatoren werden hauptsächlich zum Aufladen von Mobilfunktelefonen angeworfen. Damit bleibt die Verbindung zur städtischen Verwandtschaft konstant. Diese bildet einen Knotenpunkt, indem sie einerseits den direkten Kontakt zum Dorf hält, andererseits mittels Internet-Telefonie und sozialer Netzwerke kostengünstig mit Verwandten im Ausland verbunden bleibt.

Distanzen einer räumlichen Trennung sind daher kein entscheidender Faktor, wenn Beziehungen auseinander gehen. Gerade mit zunehmender Distanz steigt im Gegenteil das Bemühen, die Beziehung aufrecht zu erhalten. Zeitliche Dimensionen der räumlichen Trennung hingegen stellen einen bedeutenderen Einflussfaktor dar. Jedoch gelingt es auch hier zumeist, Kontakte über Jahrzehnte ohne persönliche Treffen hinweg zu erhalten.

Die sozialen Netzwerke beschränken sich stark auf Verwandtschaftsbeziehungen. Die Kartierungsmethode war nicht auf familiäre Beziehungen beschränkt, Freundschaften hätten ebenfalls verzeichnet werden können, wurden jedoch nur bei einer von über 20 Anwendungen erwähnt. Auch die städtische Bevölkerung konzentriert sich zumeist auf ihre Großfamilie. Zwar existieren intensive Freundschaften zu Arbeitskollegen oder während eines Studiums zu Kommilitonen, diese werden jedoch kaum über die Zeiten hinaus gepflegt, in denen man regelmäßigen Umgang miteinander hatte. Unabhängig ihres akuten Aufenthaltsortes, fühlen sich die Familienmitglieder einer räumlich verortbaren Gemeinschaft verbunden. Das Dorf bildet die räumliche Verankerung der eigenen Identität. Auch wenn die 
Identität räumliche Bezugspunkte hat, agiert man flexibel und zirkuliert räumlich, ohne jedoch den Bezugsrahmen der Großfamilie aufzugeben. Die Menschen nutzen daher sich im Zuge der Globalisierung bietende Chancen sich selbst zu Verwirklichen. Fidschianer, die sich ihrer Familie und dem Heimatdorf nicht verbunden fühlen, sind Ausnahmen. Dank der erweiterten Handlungsspielräume bieten sich diesen ebenfalls Gestaltungmöglichkeiten einer sinnhaften Lebensverwirklichung.

Tabelle 3: Soziale Beeinflussung individueller Handlungsspielräume*

\begin{tabular}{|l|l|l|l|l|}
\hline D1 How much impact do & \multicolumn{5}{l}{ D1.1 Do you feel satisfied with this situation? } \\
\cline { 2 - 6 } $\begin{array}{l}\text { you think you have in deci- } \\
\text { sions that affect your life? }\end{array}$ & $\begin{array}{l}\text { 1. yes, to a } \\
\text { great extent }\end{array}$ & $\begin{array}{l}\text { 2. only to a } \\
\text { small extend }\end{array}$ & $\begin{array}{l}\text { 3. no, it's a } \\
\text { bad situation }\end{array}$ & Häufigkeit \\
\hline 1. huge impact & 19 & 5 & 1 & 25 \\
\hline 2. small impact & 5 & 7 & 1 & 12 \\
\hline 3. no impact & 1 & 1 & 2 & 3 \\
\hline Häufigkeit & 25 & 13 & $\mathrm{~N}=40$ \\
\hline \multicolumn{2}{|l|}{ *basierend auf 40 interviewten Personen aus Nabuna, Koro Island (Fragebogen im Anhang) } \\
\hline
\end{tabular}

Mit den engen sozialen Beziehungen gehen Kontrollfunktionen einher. So sagen viele Fidschianer, dass sie über wichtige Entscheidungen in ihrem Leben nur wenig, teilweise gar nicht alleine bestimmen (siehe Tabelle 3). Entgegen westlichindividueller Freiheitsideale entspricht dies jedoch häufig einem Wunschzustand. So suchen viele Dorfbewohner den Rat innerhalb des Familien- und Häuptlingssystems und sind glücklich, dass sie Lasten nicht alleine tragen müssen. Gerade jüngere äußern sich, dass andere derart kluge und weitsichtige Entscheidungen über ihr Leben fällen, zu denen sie alleine nicht imstande gewesen wären. Umgekehrt erklärt mir ein Familienoberhaupt, dass er sich mehr Hilfe und Unterstützung seitens seines sozialen Umfeldes wünscht und ihn die eigene Entscheidungsmacht überfordere.

Die Familiennetzwerke tragen ungemein zur sozialen Sicherheit bei. Das Leben in der Großfamilie gestaltet nicht nur die eigene kulturelle Identität als Quelle von Lebensqualität. Neben der sozialen Verortung und emotionalen Verbundenheit tragen die familiären Strukturen mittels regelmäßiger, gegenseitiger finanzieller Hilfen auch ungemein zur ökonomischen Sicherheit bei. Da man nicht als Individuum für den eigenen Lebensunterhalt wirtschaftet, sondern in die Haushaltskasse der Kern- und Großfamilie, tragen zumeist mehrere Personen monetär zum Einkommen bei. Weiterhin stützt man sich im Regelfall auch nicht einseitig auf eine Einkommensform, z.B. Landwirtschaft, sondern erzielt auch außerhalb dieser Einkommen (siehe Tabelle 4). Diese vielseitige ökonomische Absicherung auf verschiedene Personen und Einkommensarten schafft Möglichkeiten Ausfälle zu kompensieren. Das Handeln in großfamiliären, räumlich ausgedehnten Strukturen senkt Verwundbarkeiten gegenüber sozialen Katastrophen und schafft individuelle wie soziale, menschliche Sicherheit. 
Tabelle 4: Diversität an Einkommensquellen*

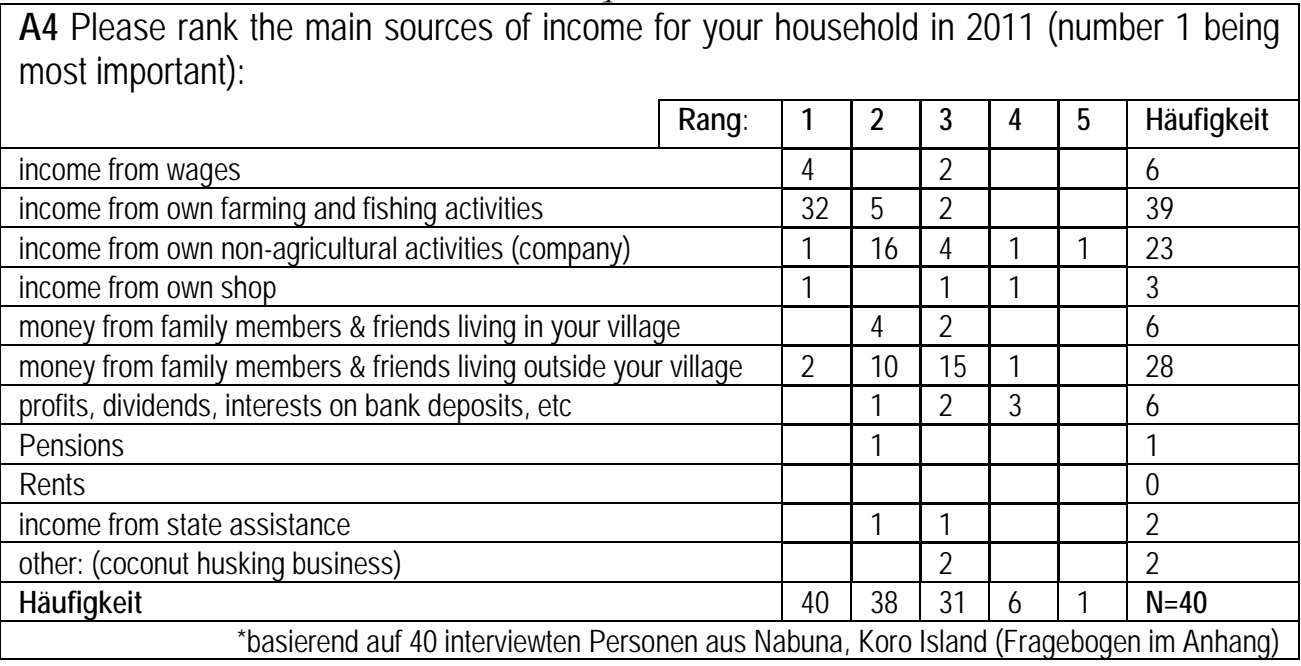




\section{Vanua - Die räumliche Manifestation der kulturellen Identität}

Das Wort vanua kann mit „Land“ übersetzt werden. Gleichzeitig bezeichnet es die obere Ordnungseinheit im Häuptlingssystem und könnte daher mit „Stamm“ übersetzt werden. Im Alltagsgebrauch jedoch können soziale Einheiten von Kleingruppen bis zur fidschianischen Gesamtpopulation unabhängig ihrer Größe als ein vanua bezeichnet werden (Ravuvu 1987: 14f.). Nach Unaisi Nabobo-Baba bezieht sich Vanua auf ,a people, their chief, their defined territory, their waterways or fishing grounds, their environment, their spirituality, their history, their epistemology and culture (2006: 155). Vakavanua, wörtlich etwa „nach Art des Landes“, könnte mit „Kultur" oder „Tradition“ ins Deutsche übertragen werden und impliziert normativ hochwertige Praktiken. Keine Übersetzungsvariante wird der Bedeutung des Wortes gerecht, da im deutschen Sprachraum die zum Verständnis notwendigen kulturellen Konzepte nicht angelegt sind. Im Folgenden erkläre ich, wie diese unterschiedlichen Bedeutungen miteinander in Einklang gebracht werden und warum das dahinterliegende sozialräumliche und spirituelle Konstrukt vanua ${ }^{54}$ für das kulturelle Selbstverständnis der Fidschianer von herausragender Bedeutung ist.

Nach Dickhardt (2001: 58) werden „unterschiedliche Strukturierungsprinzipien aus den Bereichen des Ahnenglaubens, der soziopolitischen Struktur, des Landrechts und der britischen Rechtstradition konzeptionell durch den Begriff des vanua zusammengeführt“. Öffentliche Äußerungen bezüglich Reformanregungen der Landrechtsituation können diese Totalität im Verständnis des Begriffes angreifen und haben ein enormes Potential politischer Sprengkraft. Mithilfe der lokal gewonnen Einsichten erläutere ich, wie vanua in die dörfliche Lebenswelt eingebettet ist. Diese Perspektive leistet einen Beitrag vanua ganzheitlich zu verstehen und zeigt Konfliktdimensionen der Landrechtsfrage auf die Lebensqualität der Fidschianer auf, die in der Literatur bisher vernachlässigt sind.

\subsection{Yaqona-Zeremonien}

Yaqona, auch kava oder grog genannt, ist ein Strauch, aus dem ein gleichnamiges Getränk mit leicht narkotisierender Wirkung hergestellt werden kann. Im dörflichen Kontext wird das Getränk ausschließlich ritualisiert zubereitet und in Gemeinschaft getrunken. Vom Pflanzen über die Ernte, dem Trocknen, Pulverisieren, mit Wasser Mixen bis zum Ausschenken ist der gesamte Prozess in feste, wiederkehrende Handlungen eingebunden. Veranschaulichend werde ich den Ausschank kurz wiedergeben.

\footnotetext{
${ }^{54}$ Ich gebe in dieser Arbeit keine allumfassende Darstellung des Konstruktes ab, sondern unternehme eine pointierte Beschreibung im Hinblick auf die Einbettung des Konzepts in die Grundthematik gesellschaftlicher Transformation. Dickhardt (2001) oder Ravuvu (1983), welche sich ebenfalls intensiv mit vanua beschäftigt haben, wählten entsprechend andere Darstellungen und Schwerpunktsetzungen des Konzeptes.
} 
Man sitzt im Schneidersitz in größerer Runde kreisförmig zueinander. Die tanoa, eine große Schale, wird mittig im Kreis platziert. Eine Person sitzt direkt an der Schale und schenkt aus ihr yaqona in bilo, Kokosnussschälchen, aus. Der Ausschenker sitzt in Blickrichtung gegenüber der im Häuptlingswesen ranghöchsten Person, welche das Kopfende markiert. Weitere hoch angesehene Personen sitzen ebenfalls im oberen Halbkreis um die tanoa. Die meisten Menschen sitzen hinter der tanoa mit Blick zum Obersten. Eine bis mehrere Personen fungieren als Kellner und verteilen die gefüllten bilo reihum entsprechend der Hierarchie an die weiteren Teilnehmer (siehe Abb. 12).



1 - Häuptling; 2 - angesehene Personen; 3 - Ausschenker; 4 - Kellner; 5 - tanoa; 6 - yaqona-Mix-Utensilien

Abb. 12: Schematische Sitrrunde in Yaqona-Zeremonie

Die Sitz- und Trinkordnungen drücken Respektsbekundungen aus. So setzt man sich, wenn man zu einer bestehenden Runde zustößt, für gewöhnlich auch als Person von Rang auf niedere Position und wartet, bis man aufgefordert wird, in der oberen Hälfte Platz zu nehmen. Auch sämtliche Kommunikation im Rahmen einer yaqonaZeremonie soll möglichst respektvoll gestaltet sein. Sollte aus Platzgründen der obere Sitzkreis ausgefüllt sein, verscheucht man keineswegs die Sitzenden, sondern nimmt aus höflichen und praktischen Motiven heraus eine niedere Position ein, wird jedoch in der Trinkordnung entsprechend des Rangs bewirtet. Ganz im Sinne der Theorie der Strukturierung (Giddens 1988; siehe Kap. 4.3) werden in dieser Zeremonie die Häuptlingshierarchien nicht nur beständig reproduziert, sondern zunächst vom Ausschenker und den Verteilern - ggfs. unter Zuhilfenahme weiterer Anwesenden - interpretiert, sodass es aufgrund subjektiver Wahrnehmungen zu Veränderungen der Struktur kommen kann. ${ }^{55}$

Menschen, die keine Position im fidschianischen Häuptlingswesen haben, beispielsweise Ausländer oder Indo-Fidschianer, haben zunächst keine klare Stellung in der Sitz- und Trinkordnung. Im Zuge der Transformation zollt man zudem Respekt gegenüber weltlichen Titeln und Funktionen, - beispielsweise im Bil-

\footnotetext{
55 Regional sowie je nach Anlass der Zeremonie verändern sich die Sitz- und Trinkordnungen. Die hiesige Darstellung fasst meine Eindrücke aus den Untersuchungsdörfern geschlechtsneutral zusammen. Je nach Region existieren jedoch strikte Trennungen nach Geschlechtern, wobei Frauen zumeist niedrige Positionen einnehmen (Mückler 2007: 17-19; Turner 1986: 206-208).
} 
dungs- oder Gesundheitssektor, - die nicht im Häuptlingswesen abgedeckt sind. In der alltäglichen Praxis ist der Brauch jedoch so offen gestaltet, dass Fremde in das Ordnungssystem integriert werden können. So wurde ich stets als ehrenwerter Gast aufgenommen und habe einen hochgeschätzten, fortgeschrittenen universitären Bildungsgrad, aber keinen familiären Titel und bin von jugendlichem Alter. Da die meisten Personen ein ehrliches Interesse an mir zeigten, wurde ich oftmals zu den Obersten komplimentiert um mich gut unterhalten zu können, nahm in der Trinkordnung jedoch tendenziell mittlere Positionen ein. Die Unterschiedlichkeit meiner Einordnung bei verschiedenen Gelegenheiten empfinde ich als gering. Mein Beispiel verdeutlicht einerseits die Schwierigkeiten, Respektsbekundungen im traditionalen Rahmen einwandfrei auszudrücken. Die Anwendung des Häuptlings-Hierarchiesystems birgt Konflikte, da bestimmte, neuere Werte formal nicht abgedeckt sind. Andererseits zeigt sich die Qualität des Brauches, Konflikte zu lösen. Mittels respektvoller Kommunikation wird der Konflikt im Sinne aller Anwesenden gelöst und die Lösung führt zur Integration sowohl von Fremden, als auch von neuen Werten. Diese Wandlungsprozesse werden dabei nicht als Schädigung oder Auflösung der eigenen Kultur wahrgenommen, im Gegenteil festigt die flexible Gestaltung die eigene Identität, da in der Anpassungsfähigkeit die Stärke der eigenen Kultur zum Vorschein kommt.

In nicht näher bestimmbaren früheren Zeiten waren bete für den Ausschank verantwortlich und nur Häuptlinge, Familienoberhäupter und sonstige, dem Anlass entsprechend hoch angesehene Personen durften einer yaqona-Zeremonie beiwohnen. Je nach Region blieb Frauen damit der Zugang verwehrt. Seit mehreren Jahrzehnten jedoch sind die Zeremonien eine alltägliche Praxis an der Frauen ebenfalls partizipieren und man bereits ab jugendlichem Alter teilnimmt. Als Anlässe für yaqona-Zeremonien dienen nicht mehr ausschließlich hochkulturelle Ereignisse wie Geburten, Hochzeiten und Beerdigungen oder HäuptlingsZusammenkünfte. Ihr Rahmen wurde erweitert auf Fundraising-Partys beispielsweise zum Sammeln für Schulausbildungskosten oder für kirchliche Zwecke und selbst einfache, dorfinterne Nachbarschaftsbesuche dienen als Anlass für eine Zeremonie.

Die Lethargie, die mit einem massenhaften Konsum einhergehen kann, sowie der zeitliche und finanzielle Aufwand können Ansatzpunkte für Kritik sein. Kaum ein Dorfbewohner jedoch kritisierte die Veralltäglichung des Brauches als solches, im Gegenteil trägt er zum eigenen Selbstverständnis, der eigenen kulturellen Identität und der Stärkung des Gemeinschaftsgefühls im Dorf bei und die Häufigkeit wird geschätzt. Grund hierfür ist vor allem die ritualisierte Form. Als zentral empfundene Grundelemente bleiben erhalten und die eigene Kultur wird beständig ausgelebt und gestärkt. Die Stärkung eines solchen „Wir"-Gefühls bedeutet hierbei zwangsläufig eine Abgrenzung gegenüber möglichen anderen, da die Identitätsbildung über die Unterscheidung zwischen „wir“ und „sie“ stattfindet. Die Handlung trägt damit politische Züge; einerseits werden Zusammengehörigkeitsgefühle und Kooperationen gestärkt, andererseits besteht ein Hang zum Denken 
in Freund-Feind-Kategorien (Korf 2009: 101). Aufgrund pluraler Identitäten ist ein Feindbild hierbei jedoch nicht zwingend auszumachen, so erfahren Dorfbewohner, die aus religiösen, geschmacklichen oder gesundheitlichen Gründen Verzicht üben, keine Diskriminierung. Während indisch-stämmige Fidschianer auf lokaler Ebene problemfrei integriert werden können, können sie im nationalstaatlichen Kontext jedoch mittels politischer Rhetorik zu Fremden deklariert werden.

\subsubsection{Gunu vanua - „das Land trinken“}

Das wichtigste Moment, warum die yaqona-Zeremonien zur kulturellen Identität beitragen, ist, dass sie mittels der ritualisierten Form des Trinkens eine spirituelle Dimension erhalten. Jede Zeremonie wird mit einem Gebet eingeleitet, dass den Ahnen ${ }^{56}$ gewidmet ist. Diese sind bei jeder Zeremonie anwesend. So erhalten alle Entscheidungen, die während einer yaqona-Zeremonie abgehalten werden, den Segen der Ahnen.

Die Ahnen existieren im Land, vanua, und wirken durch dieses. So besitzt jede Pflanze, jedes Lebewesen mana, eine spirituelle Kraft. Über das ritualisierte Trinken von yaqona wird eine Verbindung zu den Ahnen hergestellt (Turner 1986: 209). Häuptlinge besitzen besonders viel mana. Yaqona, welches in Gegenwart eines Häuptlings getrunken wird, heißt yaqona vakaturaga, edles bzw. königliches yaqona. Die Ahnen eines Stammes (vanua) existieren auf dem Gebiet dieses Stammes (vanua). Der Konsum von yaqona (gunu yaqona) als entscheidendes Medium zur Kommunikation mit den Ahnen wird sprachlich mitunter gleichgesetzt mit dem Konsum von vanua (gunu vanua). ${ }^{57}$

\subsubsection{Respekt gegenüber den Abnen}

Die Ahnen stehen für den Inbegriff des Lebens. Sie machen das Land fruchtbar und schaffen neues Leben. Bei einem Todesfall steigt der oder die Verstorbene am vierten Tage zu den Ahnen auf. ${ }^{58}$ Die Ahnen verkörpern damit zugleich die

\footnotetext{
${ }^{56}$ Ich benutze den Begriff der Ahnen zuvorderst im Sinne von $v u$. $V u$ sind in erster Linie Verstorbene, die namentlich noch weitestgehend bekannt sind und deren Verwandtschaftsbeziehungen zu den lebenden Dorfbewohnern nachvollzogen werden können. Weitere Geistwesen, an die die Fidschianer auch heute noch mehrheitlich glauben und die für die Verbindung zum vanua ebenfalls bedeutende Rollen spielen, sind die qase, Urahnen deren Verwandtschaftsbezug kaum noch bekannt ist, sowie degei, die alten Götter, die das vanua (den Stamm) gründeten, oder tevoro, Dämonen die das vanua heimsuchen können. Sprachlich wurde hierbei in meinen Diskussionen nicht sauber getrennt was an meinen eigenen Sprachdefiziten liegen könnte -, wobei $v u$ mitunter einen Oberbegriff darstellte, unter dem sämtliche Geistwesen subsumiert wurden.

${ }^{57}$ Nachdem mir der Ausspruch „You cannot discuss the land without drinking the land!“ (yaqona-Runde in Vatulele, Dez. 2011) zu Ohren kam, habe ich im Rahmen der Anwendung partizipativer Forschungsmethoden stets um Entschuldigung gebeten, falls der Diskussionsrahmen von gebräuchlichen Kommunikationsstrukturen abweicht.

${ }^{58} \mathrm{Im}$ Rahmen vom Kannibalismus vorchristlicher Zeiten bedeutet dies, dass man beim Verspeisen eines Menschen nicht bloß dessen mana aufnimmt, sondern ihn gleichzeitig der Rückkehr zu seinen Ahnen beraubt. Kannibalismus zielt daher wohl auf die totale Auslöschung eines verfeindeten
} 
Vergangenheit, Gegenwart und Zukunft; in spirituellem Sinne die Ewigkeit. Im vanua manifestiert sich der Kreislauf des Lebens. Vanua ist ein holistischer Begriff mit gesellschaftlichen, zeitlichen, spirituellen und physisch räumlichen Konnotationen. Den Ahnen hat man die eigene Existenz zu verdanken. Zeremoniell manifestiert sich der Dank und die Verbundenheit im Konsum von yaqona. Jeglicher Respekt untereinander spiegelt den Respekt gegenüber den Ahnen wider und jede Diskussion reflektiert die Wünsche der Ahnen. Egoismus, Falschaussagen oder Provokationen im Rahmen von yaqona-Zeremonien stellen Beleidigungen der Ahnen dar und werden aufgrund der herausragenden Bedeutung der Ahnen im Werteverständnis tunlichst unterlassen.

Die unzertrennliche Verknüpfung der Ahnen mit dem physischen Raum im Begriff vanua wird auch bei einer Grundsteinlegung im Hausbau deutlich. Hierbei ruft man ebenfalls eine yaqona-Zeremonie ein, um sich den Segen der Ahnen geben zu lassen, damit das Haus auf festem Fundament gebaut wird. Damit ist letztlich jedes Haus ein spiritueller Ort. Entsprechend groß ist die Hemmschwelle, diesen Standort beispielsweise aufgrund des Meeresspiegelanstiegs oder sonstiger Naturgefahren aufzugeben, da dies als mangelndes Vertrauen in den Schutz der Ahnen und somit als eine Beleidigung selbiger bewertet werden könnte. Nach fidschianischem Werteverständnis wäre dies eine schwere Sünde (Gespräche mit Dorfältesten in Malawai, Lamiti und Dravuni).

Während meines ersten Aufenthaltes auf Gau Island wurde die Grundschule in Lamiti mit einer Solaranlage ausgestattet. Hierfür mussten vier Löcher in die Erde gegraben werden, um Eisenträger einzulassen, welche dann einzementiert wurden. Vor dem Graben eines jeden Loches wurde eine yaqona-Zeremonie abgehalten, an der im Mindesten der Bauaufseher und der Häuptling teilnahmen, damit die Ahnen ihren Segen geben und mögliche Felsen beseitigen, die die Grabung behindert hätten.

Dieses Beispiel zeigt einerseits, dass potentielle Konflikte zwischen klassischen Vorstellungen von vanua und heutigen Bedürfnissen wie dem Bau einer Solaranlage für die Grundschule mittels Adaption gelöst werden können. Andererseits wurde ich in diesem Zusammenhang gleichzeitig Zeuge, wie die Aufnahme westlicher Denkweisen in das kulturelle Wertesystem zu Problemen führt. So zeigte sich eine Dorfbewohnerin erbost, dass das Interesse ihrer Mitstreiterinnen begrenzt war, für die Handwerker besonders große Matten als Gastgeschenke zu flechten. Da Matten insbesondere als Gastgeschenke für Hinterbliebene auf Beerdigungen Verwendung finden, kommentierte sie das Verhalten mit den Worten: „Ihr kümmert euch mehr um die Toten als um die Zukunft eurer eigenen Kinder!“ Nach einem Verständnis von Ahnen und vanua als Elemente im Kreislauf des ewigen Lebens wäre dieser Ausspruch sinnfrei.

Stammes ab - inklusive seiner Historie (eigene Interpretation nach Gesprächen in Dravuni über Tomas Baker, einem vorkolonialen Missionar aus der Region, der als wohl einziges bekanntes westliches Kannibalismus-Opfer Prominenz erlangte (Burns 1963: 85f.)). 


\subsection{Vamua im Zeichen der Transformation - Neo-traditionelle Bewegungen}

Im Rahmen der nahezu täglichen yaqona-Zeremonien reproduzieren sich die räumlichen, sozialen und spirituellen Dimensionen von vanua. Das Konzept besteht im kollektiven Gedächtnis der Gesellschaft und ist fundamental für das Selbstverständnis der Fidschianer. Seit der Nationalstaatsbildung, mit der die Kolonialisierung einherging, erhalten parallel weitere Konzeptionen von „Land“ Einzug, mit denen marktwirtschaftliche Betrachtungen als Produktionsfaktor und Fragen über Recht und Besitz aufkommen. Schon zu Beginn der Kolonialzeit wurde die Gefahr erkannt, dass moderne Vorstellungen mit der Huldigung der Ahnen nicht in Einklang gebracht werden können. Deren Untergrabung würde ein existenzielles Risiko darstellen. Im weiteren Verlauf möchte ich auch eine Reihe an Bewegungen lokaler Initiativen zur Landfrage skizzieren. Ich klassifiziere diese Strategien als neo-traditionell, da sie einerseits auf die Bewahrung solcher kultureller Werte abzielen, die als zentral für die eigene Identität wahrgenommen werden, andererseits wird sich zur Erreichung dieser Ziele „moderner“ Elemente bedient.

\subsubsection{Kolonialrechtliche Bestimmung von Land als Kollektiveigentum}

Jegliche staatlichen Eingriffe in das Landrecht stellen eine potentielle Bedrohung dar. 1876 wurde das derzeitige rechtliche Verständnis von Land als Kollektiveigentum auf mataqali-Ebene festgeschrieben. Dieses entspricht bereits nicht den Wertvorstellungen der Fidschianer. Die mataqali-Ebene ist nur eine Möglichkeit, das Gebiet der Ahnen räumlich zu konzipieren, je nach Region innerhalb Fidschis hätte man genauso gut den räumlichen Bezug zum tokatoka, yavusa oder eben vanua als soziale Gliederungsform herstellen können. Während in vorkolonialen Zeiten das Konzept von vanua lokal sozialräumlich unterschiedlich gestaltet war und flexible Handhabungen zuließ, wurden die sozialen Ordnungseinheiten im Zuge der Nationalstaatsbildung vereinheitlicht und deren physische Raumbezüge festgeschrieben.

Im Zuge der sozialräumlichen Einordnung von Land als Kollektiveigentum ist es jedoch zunächst einmal ein radikales Novum, vanua überhaupt marktwirtschaftlichen Gedankengängen zu unterwerfen. Zuvor konnte vanua kein Gut sein, welches man besitzen könne - sofern man einen Eigentümer ausfindig machen wolle, wären es am ehesten die Ahnen. Westliche Vorstellungen von Land als Besitztum - egal ob individuell oder kollektiv - widersprechen traditionellen Wertvorstellungen. Die räumliche Umwelt zuvorderst als Ressource zu betrachten, welche es marktwirtschaftlich in Wert zu setzen gilt, widerspricht dem komplexen Verständnis, welches soziokulturelle wie spirituelle Vorstellungen vom Raum beinhaltet. Die wirtschaftliche Betrachtung entzaubert und entwertet vanua. Gedankengänge über die Natur als Dienstleister für den Menschen und diesen als dessen Eigentümer zu bestimmen, könnten als anmaßend abqualifiziert werden. 


\subsubsection{Yaqona-Zeremonien zwecks Fundraising}

Über die Zeit hinweg konnte man sich als hybride Kultur jedoch mit der seitherigen Handhabung von Land als Kollektiveigentum auf mataqali-Ebene arrangieren, ohne das ganzheitlichere vanua-Konzept aufgegeben zu haben, wie die yaqonaZeremonien verdeutlichen. Dennoch unterliefen die Zeremonien einem Wandel, wie Christina Toren (1999: 37-43) basierend auf Forschungen in Sawaieke auf Gau Island von 1981 bis 1983 attestierte. So stellt sie heraus, dass im Rahmen innerdörflicher Fundraising-Aktionen den Teilnehmern gefüllte bilo zu festgelegten Beträgen gekauft werden und damit die Geldwirtschaft Einzug ins Brauchtum erhielt. Ihre Ausführungen gleichen jedoch stark meinen aktuellen Erfahrungen, sodass ich zu dem Schluss komme, dass die Art yaqona zu konsumieren zumindest innerhalb der letzten 30 Jahre nur schleichenden Veränderungen unterlag.

Die Hybridisierung der Gesellschaft durch westliche Einflüsse veranschaulicht insbesondere die Involvierung von Geld in diesem Brauch. Dadurch, dass man nicht sich selbst, sondern nur den anderen eine bilo kaufen kann, bleiben reziproke Werte enthalten. Es ist Brauch, eine bilo in einem Zug zu leeren. In den Fundraising-Runden darf man dem Beschenkten teilweise selbst einschenken, um das Schälchen bis zum Rand zu füllen. Die Einbeziehung solcher spielerischen Herausforderungen hebt die Attraktivität der Veranstaltung und das gegenseitige Aufziehen wirkt sozial integrierend.

Fundraising-Partys zur Schulausbildung werden normalerweise seitens der Eltern ausgerichtet. In Malawai jedoch hat man zum Schuljahr 2012 zum ersten mal keine individuellen Fundraising-Abende abgehalten, sondern für sechs Schulkinder und eine Studentin drei Veranstaltungen konzipiert und das eingenommene Geld in einem als fair empfundenen Schlüssel an die Eltern verteilt. Dies reduziert den Zeitaufwand und sorgt für größere Verteilungsgerechtigkeit. Innerhalb Nabunas und Vatuleles nahm ich an Fundraising-Partys teil, deren Einnahmen um den Faktor drei schwankten. Da diese Einnahmenspannen nicht unbedingt die Bedürftigkeit widerspiegeln, sondern vielmehr die Beliebtheit der Gastgeber, oder schlicht über eine günstige Terminierung zustande kommen, korrelieren die drastischen Schwankungen nicht mit dem sozialen Wertebewusstsein und sorgen für Unmut. ${ }^{59}$

Dieser Wandel in den yaqona-Zeremonien zeigt die Anpassungsfähigkeit der Gesellschaft. Hier bestätigt sich Christina Torens Verständnis von Tradition als Prozess, welcher es über die Zeit ermöglicht, Veränderungen als kulturelle Elemente ohne einen Identitätsverlust in die Gesellschaft zu integrieren (1988: 697f.).

\footnotetext{
${ }^{59}$ Im Sinne der entwicklungspraktischen Leitbilder der partizipativen Forschung informierte ich im Anschluss an meine Erfahrungen auf Gau Island die Gemeinden in Nabuna und Dravuni über das neue Konzept in Malawai. Eine Beurteilung, ob diese Handhabung im örtlichen Werteverständnis ausschließlich positiv aufgenommen würde und sie somit auch dort eingeführt werden sollte, steht mir nicht zu. Allerdings möchte ich meine Position in den Gemeinden dazu verwenden, kulturell angepasstes Wissen zu verbreiten um erweiterte Handlungsmöglichkeiten aufzuzeigen. Inwiefern diese genutzt werden, muss einer jeden Gemeinde jedoch frei überlassen bleiben.
} 
Insbesondere weil sich die Veränderungen nur schleichend vollziehen, bewahren sich sowohl der als traditionell empfundene und damit identitätsstiftende Charakter, als auch die Anpassungsfähigkeit, trotz des gesellschaftlichen Wandels fortzubestehen. Das Konzept von vanua wurde nicht verdrängt, sondern hat im yaqonaKonsum eine Renaissance erfahren, Dank welcher bestimmte traditionelle Werte über die turbulenten Zeiten hinweg bewahrt werden konnten. Im Sinne von vakavanua, die Traditionen explizit dynamische Züge andenkt, werden die Zeremonien seitens der Bevölkerung jederzeit als traditionell wahrgenommen, da stets als wesentlich empfundene, Identität reproduzierende Elemente erhalten bleiben.

\subsubsection{Vereinbarkeit des christlichen Glaubens mit vanua}

Auch der christliche Glaube ist mit den Vorstellungen von vanua vereinbar. Ich kann in dieser Arbeit die Komplexität des vanua Konzeptes nicht ganzheitlich entschlüsseln, geschweige denn die vielschichtigen Verbindungen zwischen dem christlichen Glauben und weiteren kulturellen Wertvorstellungen ganzheitlich betrachten. An dieser Stelle werden weder die historisch gewachsenen Prozesse umfassend nachgezeichnet, noch die Vielschichtigkeit in ihrer regionalen Fülle wiedergegeben. Stattdessen veranschauliche ich an ausgewählten Beispielen Wege, die bestritten werden, den christlichen Glauben in Einklang mit vanua zu bringen.

Zentrale Stellen der Bibel können so interpretiert werden, dass sie auf die Vorstellungen von vanua verweisen. An verschiedensten Stellen der Bibel ist von Geistwesen die Rede, zumeist Engel oder der Teufel. Die Vorstellung von übersinnlichen Ahnen und tevoro, Teufeln oder Dämonen bildet damit keinen zwingenden Widerspruch. Menschen sowie das vanua können von rastlosen Dämonen heimgesucht werden, so wie Jesus vom Teufel versucht wurde (Mt 4, 1-11; Lk 4, 1-13). Jedoch ist akzeptiert, dass der christliche Gott der mit Abstand mächtigste ist. Auch Vorstellungen von mana, jener göttlichen Kraft, die durch die Ahnen im vanua existiert, kann als von der Bibel abgesegnet betrachtet werden: So heißt es im vierten Gebot „Ehre deinen Vater und deine Mutter, damit du lange lebst in dem Land, das der Herr, dein Gott, dir gibt“" (Ex 20, 12). Die gesamte Praxis der Verehrung der Ahnen kann in diesem Gebot ihre Legitimation finden.

Insbesondere die methodistische Kirche hat seit den Anfängen der Missionierung einen engen Bezug zum Häuptlingswesen und zum vanua aufgesucht, worin ihr Erfolg begründet sein könnte. So wird das Häuptlingstum als gottgegebene Ordnung verstanden, der Zufolge Häuptlinge oftmals Halbgöttern gleichgestellt werden, durch die besonders viel mana fließe. Die yaqona-Zeremonien zur Verehrung der Ahnen werden nicht unterbunden, sondern aktiv seitens der religiösen Vertreter unterstützt, indem sie mit christlichen Gebeten unterlegt werden, sodass die Zeremonien eingebettet sind in die christliche Lehre. Im Sinne von vakavanua ersetzt der christliche Glaube nicht die alten Wertvorstellungen, sondern entwickelt diese weiter. Die Zeremonien bleiben authentisch, über das kulturelle Verständnis von Tradition als Prozess kann die Identität gewahrt werden (Dickhardt 
2001: 96-101). In Dravuni erhielt ich folgende Erklärung, wie die vorkoloniale Vergangenheit und der christliche Glaube in Einklang gebracht werden können:

Viele sagen, vor der Ankunft der Missionare wären wir Heiden, wilde Menschenfresser ohne Werte. Die Missionare hätten uns die Schrift und die frohe Botschaft gebracht. Aber die frohe Botschaft war bereits da. Wir waren keine Wilden. Wie sonst hätten wir friedlich Kontakt zu den Fremden aufgenommen, zugebört, und binnen so kurzer Zeit den Glauben in uns aufnebmen können? Wir kannten Gottes Wort bereits bevor die Bibel zu uns gebracht wurde. (ehemalige Grundschullehrerin, Dravuni, 72)

Von einer postkolonialen Perspektive aus sind den dominanten Positionen der Kolonialherren abwertende und erniedrigende Ansichten der dominierten Kulturen inhärent; im Sinne des Eurozentrismus verachteten die christlichen Missionare die pazifischen Kulturen als wild, wollüstig und barbarisch. Dies hat einen nachhaltenden Effekt auf die unterdrückten Gesellschaften, weshalb Fidschianer ihre Geschichte oftmals spalten in ein dunkles Zeitalter der Barbarei, gefolgt vom Zeitalter der Erleuchtung durch Christentum und Zivilisation (Hau'ofa 1993: 3). Diesem latenten Konflikt, der sich negativ auf die eigene Herkunft bzw. Identität und damit das Selbstwertgefühl niederschlägt, wird mittels der Argumentation im obigen Zitat ebenfalls entgegengewirkt.

\subsubsection{Wirtschaftliche Inwertsetzung des Landes - Zugang zu Land als Ressource}

Seit Jahrzehnten wird öffentlich diskutiert, das bestehende Landrecht Veränderungen zu unterwerfen. So können Nicht-Fidschianer vergleichsweise wenig vom Land als Ressource profitieren, weshalb Ideen von Land als individualisiertem Besitz geäußert werden. Dies schürt Unsicherheiten seitens der Fidschianer, wie ihre Werte dann noch in adäquater Form ausgelebt werden könnten. Ein Ausbleiben der Auslebung entspräche einer Verschlechterung der Lebensqualität. Aufgrund des geringen Verständnisses über staatliche Funktionsweisen und verfassungsrechtliche Grundrechte können die Existenzängste politisch zu Ressentiments gegen „Fremde“ aufgewiegelt werden. Insbesondere die häufigen Putsche, Machtwechsel (ob durch Wahl oder Putsch) und Verfassungsänderungen verstärken diese Unsicherheit. Im Sinne der Ideale freier Meinungsäußerung und Verwirklichungschancen ist es für die Gesellschaft insgesamt positiv, dass Stimmen für eine Veränderung des Landrechts in die Öffentlichkeit getragen werden. Jedoch greift die Diskussion zu kurz, als dass ein echter öffentlicher Vernunftgebrauch stattfände. Die Kernargumente beschränken sich auf wirtschaftliche Dimensionen. Befürworter für eine Veränderung des Landrechts plädieren für einen gerechteren Zugang zur Ressource Land, da indisch-stämmige Fidschianer de facto vom Besitz ausgeschlossen sind, und attestieren ökonomischen Aufschwung, sofern Investoren dieser Zugang ermöglicht würde. Ein verbreitetes wirtschaftliches Gegenargument wäre, dass die indisch-stämmige Bevölkerung bereits jetzt die wirtschaftliche Elite des Landes stelle und die Bevorzugung der Fidschianer 
beim Landrecht einen sozioökonomischen Ausgleich darstelle (Lal 2003). Solange das kulturhistorische und spirituelle Konzept von vanua keine prominente Stellung im gesamtstaatlichen Diskurs erhält, bleibt die Argumentation unvollständig und der Konflikt nicht lösbar.

Nichtsdestotrotz wird das fruchtbare Land der Ahnen nicht nur subsistent zur Versorgung der eigenen Gemeinde bewirtschaftet und zum reziproken, unentgeltlichen Tausch mit Nachbargemeinden verwendet, sondern es wird zusätzlich in marktwirtschaftlichen Strukturen gearbeitet.

Mangels persönlicher Kontakte zum Absatzmarkt können die Bewohner Nabunas auf Koro Island selten Matten verkaufen, jedoch haben sich regelmäßige, verlässliche und günstige Transportbedingungen etabliert, sodass auch Grundnahrungsmittel, vor allem dalo und kassava für den Markt in Suva angebaut werden. Die Plantagen für den kommerziellen Verkauf weisen dabei eine geringere Diversität an Kulturpflanzen auf kleinstem Raum auf, als Gärten zum Eigenbedarf. Landkrebse werden dem Marktgeschehen wie erwähnt zur Stärkung soziokultureller Bindungen entzogen. Ein Mitglied der Gemeinde Nabunas führt einen yaqona-Verkaufsstand im Markt. Diese Vertrauensperson wird sehr geschätzt (bedeutende Personen-Auflistung der Farmer). Denn der Markthandel in Fidschi stellt keine reine unpersönliche, geldbasierte Tauschbeziehung dar, sondern lebt von persönlichen Kontakten. Sowohl zwischen den Produzenten, Transporteuren und Händlern, als auch abgeschwächt zu den Konsumenten bestehen im Regelfall soziale Beziehungen, aus denen die marktwirtschaftlichen Beziehungen erst hervorgingen. Der Verkaufsstandbesitzer garantiert aufgrund seiner sozialen Verbundenheit die Abnahme von yaqona seitens seiner Angehörigen. Zudem verkauft er ausschließlich yaqona von Koro Island, welche als Bestandteil Lomaivitis einen Ruf exzellenter Qualität genießt. Er hat einen großen Kundenstamm an Städtern, deren Heimat Koro ist, und mit denen er aufgrund der Heimatverbundenheit private Kontakte pflegt. Darüber hinaus wird er auch von Kunden aufgesucht, deren Herkunftsort kein top-Anbaugebiet ist.

Die Einwohner Dravunis haben vergleichsweise marginale Transportkosten und können alle Agrarprodukte kostengünstig zum Markt bringen. Ihnen fehlt jedoch eine persönliche Kontakt- und Vertrauensperson, sodass sie ihren Zugang zum Markt als höchst eingeschränkt empfinden (Gespräche zu Venn-Diagramm, siehe Abb. 13). Zumeist bringen sie ihre Produkte auf Verdacht zum Markt und sind auf die Gunst der Händler angewiesen, ihre Produkte zu fairen Marktpreisen abzunehmen.

Alle drei Hauptuntersuchungsgebiete verfügen über mehr potentielle Anbaufläche, als sie selbst bewirtschaften. Auf Gau und Koro Island bestehen daher weitestgehend intakte Regenwälder. In Malawai hat die Aufteilung des Landes auf die drei mataqali nur geringe Bedeutung. Die einzelnen Farmer bewirtschaften eine Vielzahl kleiner Felder, die divers im vanua verteilt sind, damit man entsprechend der Anforderungen der jeweiligen Kulturpflanzen sowohl im Flachen als auch in der Höhe, in Ebenen wie an Hängen und in sumpfigen wie in trockenen Räumen 
stets optimale Flächen nutzt. Damit die Ältesten ihr arbeitsfähiges Alter ausweiten können, ist ihnen das dem Dorf nächstgelegene Farmland vorbehalten, welches formal zum mataqali Nauluvatu gehört. Für den Zugang zu Land wird in entsprechenden traditionellen Zeremonien das jeweilige mataqali-Oberhaupt gefragt, unabhängig ob man Mitglied seines mataqali ist. Bedeutende Geschenke sind dabei im Gegensatz zu anderen Regionen Fidschis nicht vorgesehen. Die Abgabe eines Teils der Ernte ist gängiger Brauch, welche das Oberhaupt jedoch nicht für sich selbst einbehält, sondern an Bedürftige der Gemeinschaft verteilt, sodass sich hierüber soziale Sicherheitssysteme etablieren.

Aufgrund der Fährverbindung nach Suva und Savusavu kann das Land Koro Islands wirtschaftlich in Wert gesetzt werden. Daher lassen sich seit den 1960er Jahren sporadisch Fidschianer entlegener Inseln auf Koro nieder, so auch die Einwohner der Siedlung Nagadro auf dem Gebiet Nabunas. Auf Koro existieren auch kleine Minderheiten an indisch-stämmigen Fidschianern und Rotumanen. Sie pachten ihre Nutzflächen bei den entsprechenden mataqali über das TLTB als formale Vermittlungsbehörde. Aufgrund dieser Möglichkeiten einer finanziellen Inwertsetzung des Landes verpachten sich die Dorfbewohner verschiedener mataqali gegenseitig das Land ebenfalls unter Zuhilfenahme des TLTB zu gleichen Konditionen. Insbesondere der einwohnerstarke mataqali Sawana, welcher jedoch kaum eigenes Land besitzt (siehe Karte 4), pachtet Farmland. Die vielseitigen sozialen Beziehungen werden durch diese rein monetäre Verbindung belastet, da hier nicht mehr den Werten entsprechend das sozial Verbindende, sich gegenseitig Aushelfende und einander Respektierende im Vordergrund steht, sondern eigener Profit auf Kosten des anderen. Gerade in dem Wissen um traditionelle Handhabungen, wie sie andernorts auch aktuell noch praktiziert werden, betrübt die Pächter das Verhalten der Verpächter, wie mir in Zwiegesprächen anvertraut wurde. Eine offene Austragung des Konfliktes scheint jedoch vermieden zu werden, um das soziale Miteinander im alltäglichen Dorfleben intakt zu halten. Gerade über die Abwiegelung des Geschäftlichen über das TLTB scheint man einen direkten Konflikt umgehen zu können, aufgrund des Vermittlungsgebühren des TLTB und des bis 2012 praktizierten Verteilungsschlüssels der Einnahmen auf Häuptlinge verbleit jedoch ein Teil der Summen nicht in der Gemeinschaft.

Neben dem Disput über soziale Werte kann die Beschränkung auf Farmland des eigenen mataqali und zusammenhängenden Pachtland-Flächen zu einer geringeren Vielfalt an Nutzflächenformen im Repertoire des Einzelnen sorgen. Traditionelles Wissen um Nutzpflanzenvielfalt und deren Anbaumethoden könnte wegen der begrenzten Diversität verloren gehen, was insbesondere im Hinblick auf die Anpassung an Naturgefahren bedenklich ist. Diese Gefahr wird jedoch dadurch gemindert, dass die Jugend zumeist in Kleingruppen unterschiedlicher mataqali sämtliche Felder der Gruppenmitglieder reihum bewirtschaftet, sodass die Weitergabe des Wissens um eine Vielfalt an Techniken gewahrt scheint.

$\mathrm{Da}$ in Dravuni die Flächen beider mataqali eine ausreichende Größe und Heterogenität besitzen, nutzen die wenigen Farmer im Wesentlichen das ihnen zugehö- 
rige Land. Eine zeremonielle Praxis, nach der man den Zugang zu Land erbittet, wird aufgrund des fehlenden Häuptlings nicht ausgeübt. Die Farmer sprechen sich schlicht untereinander ab. Mangels des Häuptlings zur sozialen Umverteilung, entschieden sich mehrere der Farmer sozial schwächere Familien mitzuversorgen. ${ }^{60}$ Für Gemeinschaftsprojekte wie die Ingwer-Plantage wurde ein von der Straße aus zugängliches Feld bestellt, bei den praktischen Überlegungen hat die mataqali-Zugehörigkeit allenfalls eine untergeordnete Bedeutung gespielt. Ob man Teile des Landes als Pacht zur Verfügung stellen soll, wird immer wieder mal andiskutiert. Jedoch kann sich hierfür bisher kein breiter Konsens finden, da man die spirituellen und sozialen Risiken, die bei einer deutlicheren marktwirtschaftlichen Einverleibung des vanua drohen könnten, nicht abschätzen kann und daher scheut.

\subsubsection{Touristische Niederlassung auf Koro Island}

Tourismus ist Fidschis größter Wirtschaftszweig. Dieser konzentriert sich auf die Yasawa Inselgruppe, auf die West- und Südküste Viti Levus und auf Taveuni (siehe Karte 1). Doch auch auf Koro Island konnte sich ein Ressort niederlassen (Fink 2014), das ich während meiner Aufenthalte in Nabuna dreimalig aufsuchte. Dieser Standort begründet sich aus der historischen Entwicklung des Landrechts. Im 19. Jahrhundert stand Koro Island unter dem Herrschaftseinfluss von Ratu Seru Cakobau (Gravelle 1988: 46; siehe Kap. 5.1 .2 \& 6.3.3). Um Schulden für ein Dampfschiff als Kriegsgerät gegenüber einer amerikanischen Treuhandgesellschaft zu begleichen, verpfändete Cakobau einen Landstrich im Nordwesten Koro Islands, auf dem sich die Dorfgemeinschaft Nabunas befand, damals noch Matana Levu genannt (Bayliss-Smith 1976: 11f.; Burns 1963: 70). Im Zuge der Landreform zur Kolonialzeit wurde das Gebiet zu freiem Grundbesitz erklärt (siehe Karte 4). Dieses Stück Land kann daher gekauft werden und muss nicht gepachtet werden, was es für ausländische Investoren interessant macht. In Kombination mit der regelmäßigen Fähranbindung Koro Islands ist das Grundstück attraktiv genug, dass in der Bucht Dere Bay ein von australischen Investoren aus betriebenes Touristenressort aufgezogen wurde. Insbesondere der angrenzende mataqali Naduruniu hegt jedoch historisch begründete Besitzansprüche, sodass ein Konfliktfeld besteht.

Ratu Cakobau veräußerte jedoch nur das Land, die vorgelagerte Küstenzone verblieb als qoliqoli der exklusive Fischgrund Nabunas - sowie Nabasovis für den Südteil der Bucht. Da die Touristen die Bucht nutzen wollen, besteht beiderseits ein Kommunikationsbedarf und Nabuna hat eine gute Verhandlungsgrundlage. Seit der Gründung im Jahr 2000 wird das Ressort vor Ort von einem Fidschianer verwaltet. Dieser aus Koro stammende Verwalter genießt hohes Vertrauen seitens der australischen Investoren, der ausländischen Touristen und der lokalen Bevöl-

\footnotetext{
${ }^{60} \mathrm{Da}$ man nach christlichen Wertvorstellungen nicht mit den eigenen Spenden prahlt, sondern im Gegenteil versteckt gute Taten vollbringen möchte (Mt 6, 1-4), sind mir die Dimensionen, wer wieviel umverteilt, verborgen geblieben.
} 
kerung. Als Mittels- und Vertrauensmann konnte er so eine Kommunikationskultur aufbauen, die die Konflikte löst. Er versteht es, die Anliegen der jeweiligen Partei den anderen soweit zu vermitteln, dass gegenseitiges Verständnis und Akzeptanz den Umgang kennzeichnen. Gerade in der fidschianischen Kultur stößt die indirekte Kommunikation über einen Mittelsmann bzw. Fürsprecher auf Wohlwollen (siehe Exkurs 1 und Kap. 1.4). Von Anfang an war die Touristenanlage auf eine Einbettung im Inselleben bedacht und lud beispielsweise zur Einweihung die Häuptlinge aller 14 Dörfer Koros ein. Inzwischen sind die Kommunikationswege etabliert, sodass die Anliegen der Touristen und Dorfbewohner gegenseitig Gehör finden und über diesen öffentlichen Vernunftgebrauch eine Kommunikationskultur der Konfliktlösung entsteht.

Für die Errichtung eines Anlegestegs und die Nutzung der Dere Bay wurde so unter Wahrung fidschianischer Etikette in den beiden angrenzenden Dorfgemeinschaften um Erlaubnis gefragt und es werden Abgaben errichtet. Dies stößt auf große Akzeptanz. Zudem können mehrere Fidschianer einer bezahlten Tätigkeit nachgehen, beispielsweise als Gärtner, Küchen- oder Haushaltshilfe.

Insgesamt besteht das Ressort aus etwa 50 Häusern, die fast ausschließlich als Ferienhäuser Dauergästen gehören und die meiste Zeit im Jahr leerstehen. Aufgrund dieser Kleinheit sind die Auswirkungen auf die Gemeinschaften begrenzt. Da die Touristen keine einmaligen Kurzurlauber sind, sondern ein langfristiges Interesse an dem Ort haben, setzen sie sich für eine nachhaltige Nutzung der Korallenbucht ein. Weil die Dorfbewohner ebenso an einem gesunden Korallenriff interessiert sind, überschneiden sich die Interessen. Über den Mittelsmann kommt es so zu einem fruchtbaren Informationsaustausch über den Erhalt des Gebietes. Dank des Verwalters als Schlüsselfigur, die von allen beteiligten Akteuren Vertrauen und Wertschätzung genießt, konnte über kulturell eingebettete Kommunikationswege öffentlicher Vernunftgebrauch angeregt werden. Trotz einer denkbar ungünstigen, konfliktträchtigen Ausgangslage um Landtitel und rechte stellt die Touristenanlage so insgesamt eine Bereicherung für die Dorfbewohner dar.

\subsubsection{Generationenkonflikt beim Reinigen der Friedhöfe}

Ein hoher Festtag in allen besuchten fidschianischen Gemeinden ist die Reinigung der Friedhöfe zu Ehren der Ahnen, jeweils wenige Wochen vor Weihnachten. Regional unterschiedlich sieht der grobe Ablauf vor, dass man eine kurze yaqonaZeremonie abhält, bei der die ausschließlich männlichen Friedhofsgärtner je eine einzelne bilo trinken, um hierbei den Segen der Ahnen zu erhalten, auf der Grabstätte arbeiten zu dürfen. Während die Männer der Reinigung nachgehen, schmücken die Frauen die Gemeindehalle und treffen sonstige Vorbereitungen für ein großes Fest. Nach der Reinigung findet in der Gemeindehalle eine große Zeremonie zu Ehren der Ahnen statt. 
In Dravuni gab es jedoch unterschiedliche Wertvorstellungen darüber, welche Praktiken im Rahmen der Friedhofsreinigung und des Anschließenden Festes zielführend seien (siehe Exkurs 4) Die Jugend wollte sich zum einen gerne moderner Hilfsmittel bei der Reinigung bedienen. Weiterhin wollte sie mehr Zeit in die Vorbereitung des festes zur Huldigung der Ahnen investieren. Die Ältesten beharrten jedoch darauf, keine Abweichungen zur bisher gängigen Praxis zu erlauben. Die Jugend setzte hierbei ihren Willen partiell durch, indem sie Hilfsmittel verwendete. Daraufhin wurde das Fest selber jedoch abgesagt.

Der Friedhofskonflikt, welcher in diesem Fallbeispiel zugleich als Generationenkonflikt verstanden werden kann, verdeutlicht die soziale Sprengkraft der gesellschaftlichen Transformation. Obwohl der Grundwert, die Ahnen zu preisen, generationsübergreifend unangetastet bleibt, divergieren die Ansichten, wie man diesem gemeinsamen Ideal am besten gerecht wird. In Ergebnis verhindern die ungelösten Meinungsverschiedenheiten, dass das Ideal ausgelebt wird. Eine Kommunikationsplattform, auf der dieser Konfilkt hätte gelöst werden können, bevor er eskalierte, ist nicht gefunden worden. In Gesprächen mit mir zeigten die beiden lager Einsicht und verständnis in die Ansichten der jeweils anderen. Untereinander wurde dies jedoch nur mangelhaft kommuniziert, sodass öffentlicher Vernunftgebrauch litt. Die Gemeinschaft erreicht in diesem Beispiel ihre Ziele nicht, zumindest temporär leidet Lebensqualität.

Damit der Riss sich nicht ausweitet und die Gemeinschaft weiter zerbricht, sucht man Halt in weiteren gemeinsamen Werten und Idealen, in diesem Fall in der christlichen Tradition. Mittels christlicher Nächstenliebe und dem Hochhalten der Konstruktion einer Gemeinschaft über Weihnachten wird aktiv soziale Sicherung betrieben. Durch die Reproduktion verbindender Werte wird dem Schaden der Transformation entgegengewirkt. Würde es im Zuge der Transformation zu einem Auseinanderdriften der Grundwerte kommen, wäre man der Basis einer kulturellen Gemeinschaft beraubt. Im Angesicht eben dieser Gefährdung durch die wertetransformierenden globalisierten Einflüsse auf die Gesellschaft wehrt man sich mittels eines aktiven Hochhaltens der eigenen Kultur zur Stärkung der gemeinsamen traditionellen Grundwertebasis. Diese aktive Verfestigung der Werte stellt eine neo-traditionelle Gegenbewegung zur Schwächung der gesellschaftlichen Transformation dar. Ebenso kann das bewusste Unterlassen, Land als Quelle für Pachteinnahmen zu nutzen, als neo-traditioneller Versuch der Erstarkung des Grundwertes von vanua gedeutet werden.

\subsubsection{Traditionelle Fischtreibjagd als Gegenbewegung}

Die neo-traditionellen Gegenbewegungen in Dravuni sind kein rein lokales Phänomen. Die Inflation an yaqona-Zeremonien stellt eine teils bewusste, teils unbewusste (Wieder-) Erstarkung der als traditionell und soziokulturell verbindend konstruierten Werte innerhalb der gesamten fidschianischen Gesellschaft dar. Wie ich am Beispiel einer traditionellen Fischtreibjagd, der ich in Malawai beiwohnen 
durfte (Fink 2012), verdeutlichen will, beschränken sich die Versuche einer bewussten Gegensteuerung der Transformation nicht auf besondere Regionen innerhalb Fidschis. Auch wenn sie lokalspezifischen Ausprägungen unterliegen und untereinander nicht immer vernetzt sind, stellen die neo-traditionellen Gegenbewegungen gesamt-gesellschaftliche Phänomene sozialer Sicherung dar, sowohl im Dunstraum der Städte als Motoren der Transformation, wo Dravuni zu verorten ist, als auch in der entlegenen Peripherie Gau Islands.

Der yavirau (traditionelle Fischtreibjagd) findet in einem ritualisierten Kontext zur Weihnachtszeit statt. Beschlossen wird er auf einem boca ni vanua (traditionelle Gemeindeversammlung), von den beiden mataqali bati (Krieger-Klan). Die Vorbereitungen beginnen Anfang Dezember mit der Säuberung aller Gräber auf den Friedhöfen, um den Segen der Ahnen zu empfangen und dadurch einen guten Fang zu erzielen. Als dies einmal nur halbherzig ausgeübt wurde, so wird mir berichtet, war das Fangergebnis katastrophal. So wird ein direkter Zusammenhang zwischen dem yavirau und vanua konstruiert, welcher die folgenden ritualisierten Abläufe und begleitende Reproduktionen gesellschaftlicher Ordnung legitimiert.

Die technische Grundidee der Fischtreibjagd beinhaltet ein kilometerlanges Seil, das kreisförmig auf dem Riff ausgelegt wird. Langsam aber geräuschvoll wird der Kreis wie eine Schlinge enger gezogen und die Fische in die Kreismitte gedrängt, wo sie bei Ebbe eine leichte Beute darstellen. Der yavirau, dem ich 2009 beiwohnte, fand auf Heilig Abend statt. Eine Woche vorher wurden Lianen von sämtlichen Männern gesammelt und zu 2 Seilen aneinandergebunden, bis die Ältesten entschieden, dass sie lang genug seien. Am Tag vor Heilig Abend wurden Wedel von Kokosnusspalmen abgeschlagen, der Länge nach halbiert und um das Seil gewickelt, wobei auch Frauen und Kinder halfen. Dies wird so kurzfristig gemacht, damit die Palmwedel frisch bleiben und später geräuschvoll durchs Wasser gezogen werden können um die Fische zu hetzen. Am Tag selbst werden zwei motorisierte Fischerboote mit den Seilen beladen. Bevor sie bei Flut auf das Riff hinausfahren, werden die Seile noch mit den Blättern des giftigen sinu-Baumes (Excoecaria agallocha) geschlagen, um Unglück zu vermeiden. Schwangere Frauen dürfen nicht am yavirau teilnehmen, da sie Pech in Form von Fischattacken oder Ertrinken bringen. Insbesondere verheimlichte Schwangerschaften sind mächtiger als die sinu-Blätter. Aus rein praktischen Gründen dürfen eigentlich keine Kinder und Nachzügler mitmachen, um den Ablauf nicht zu stören. Für letztere wurde im von mir teilgenommenen Fall jedoch eine Ausnahme gemacht.

Jeder Teilnehmer hat bei der Treibjagd seine spezifische Rolle. Die bati mataqali sind für die nördliche Hälfte zuständig, der zahlenmäßig größere mataqali Nauluvatu (Häuptlingsklan) für die südliche. In früheren Zeiten wurden Positionierungsfehler und sonstige Verstöße mit Stockhieben bestraft, heutzutage kommen drakonische Strafen jedoch nicht mehr vor. Als Gast kann ich mir meine Position aussuchen, genauso wie Besucher aus Lamiti. Die Gesamtaufsicht hat eigentlich der Tuinimata (Sprecher des Häuptlings) inne. Da dieser in meinem Fall jedoch verhindert ist, übernimmt der derzeitige Interimshäuptling die Führung. Er 
ist der einzige, der während des yavirau sprechen darf. Er fährt das Seil entlang und gibt Anweisungen, wie der Kreis enger gezogen werden muss, damit man am Ende auf einem möglichst flachen Teil des Riffs auskommt, ohne dass Fische entweichen. Das Gebot zu Schweigen findet allerdings während des gesamten yaviran wenig Beachtung.

Gegen Ende der Treibjagd bei Ebbe sind die Fische schließlich auf engstem Raum zusammengepfercht und die Teilnehmer bilden eine nahezu undurchdringliche Mauer darum. Der Tui gibt nun Anweisungen, sich hinzusetzen, damit die Fische nicht verschrecken und versuchen auszubrechen. In der beobachteten Praxis standen jedoch viele Teilnehmer auf, um nach fünf Stunden im Wasser der Kälte zu entfliehen. Die Ältesten des tokatoka des Tuinimata sahen es daher als notwendig an, die Anweisungen des Häuptlings fortlaufend zu wiederholen. Mit Speeren, Buschmessern oder den bloßen Händen werden auf Kommando die größeren Fische gefangen und ins Boot verfrachtet. Anschließend werden die kleineren Fische mit Netzen gefischt. Wenn die letzten Fische gefangen sind und die Treibjagd zu Ende geht, werden die Fische zum Dorfplatz gebracht und dort unter den folgenden Gruppen verteilt:

- die Mitglieder der drei mataqali

- der Priester

- der Tui Malawai

- Besucher

- Farmer der mataqali, die Lianen und Kokosnusswedel stellten

- die Bootsbesitzer

Hierbei wird beachtet, dass сити (Drückerfisch, Familie der Balistidae) nicht vom mataqali Nauluvatu verspeist werden darf, sondern den Kriegern vorbehalten ist. Ein Gast darf zudem als tawvu (Freund) oder vasu (von mütterlicher Verwandtschaftslinie) - wie bei anderen Traditionen auch - sämtliche Regeln brechen und so viel Fisch für sich beanspruchen, wie er tragen kann. Dieses Privileg wird aber nicht ausgenutzt und die Aufteilung verläuft harmonisch. Aufgrund der unterschiedlichen Leistungen und Verantwortungen, die die jeweiligen Gruppen übernehmen, wird die unterschiedliche Aufteilung als fair empfunden.

Der Fisch wird ausschließlich gemeinschaftlich zubereitet und verspeist. Zudem dient er als Gastgeschenk bei der Vielzahl an gegenseitigen Besuchen, die über Weihnachten zwischen den Dörfern und innerhalb der Gemeinschaften stattfinden. Gemeinschaftssinn und reziproke Werte werden an diesen Tagen besondern deutlich ausgelebt.

Nicht nur zeitlich an Heilig Abend wird der Brauch in den christlichen Glauben eingebettet. Während des yavirau wurden mehrfach kurze gemeinsame Gebete abgehalten bevor am späten Abend die Weihnachtsmesse gehalten wird. In meinem Beisein gingen die Methodisten, welche die größte Konfession bilden, in ihre Kirche, die zwei Mitglieder der Seventh Day Adventists schlossen sich ihnen an, da ihr Gotteshaus in Vanuaso an dem ereignisreichen Tag zu weit entfernt scheint. 
Die Mitglieder der dritten Konfession des Dorfes, All Nations, feierten in ihrer Kapelle gut $200 \mathrm{~m}$ außerhalb der Siedlung.

Nicht nur Glaubensvorstellungen, soziokulturelle Geschlechterrollen, Klanstrukturen und Altershierarchien werden in dem Brauch reproduziert, sondern damit einhergehend auch Wertvorstellungen. Insbesondere auf die Attraktivität des Brauches ist man bedacht, weshalb verschiedenste Ausnahmen und Missachtungen gegenüber einem idealen Ablaufes gewährt werden. So lockt dieses Ereignis die außerhalb Malawais lebende Verwandtschaft an und frischt damit die engen Familienverbünde auf und die sozialen Netzwerke werden gestärkt. Eine Einwohnerin sagte, der yavirau sei oftmals der ausschlaggebende Punkt, warum die auswärts lebenden Familienmitglieder trotz der logistischen und finanziellen Herausforderungen ihren Weihnachtsurlaub im Heimatdorf verbringen. Der yavirau stellt somit ein Paradebeispiel des Neotraditionalismus dar. Einerseits werden traditionelle Praktiken und Werte im Ritual reproduziert. Andererseits werden diese aktiv im Sinne neumodischer Attraktivitätsverständnisse justiert. Die dahinterliegende Motivation liegt in der Steuerung aktueller, transformierender Trends; hier die Wahrung des Zusammenhaltes einer als soziokulturelle Einheit konstruierten, räumlich fragmentierten Gruppe.

\subsubsection{Monasavu: Besetzung eines Staudammprojektes}

Eine neo-traditionelle Graswurzelbewegung im Hochland Viti Levus erhielt aufgrund ihrer Gewaltdimensionen besondere mediale Aufmerksamkeit. Im Juli 1998 besetzten 3.000 Dorfbewohner das staatliche Wasserkraftwerk Monasavu, das auf ihrem vanua errichtet wurde. Sie präsentierten sich dabei vor Kameras in traditioneller Krieger-Kluft, angemalt und mit Speeren und Knüppeln bewaffnet (Abramson 2004: 76-79).

Dieses Bild, das an vorkoloniale Kriegszeiten erinnern sollte, veranschaulicht besonders deutlich die Komplexität und intrinsische Risiken neo-traditioneller Gegenbewegungen. Es zeigt die Widersprüche und Paradoxien auf, denen hybride Kulturen schnell unterliegen können. So existiert ein Streben, selbstbestimmt eigene Pfade beschreiten zu können, ohne sich jedoch zwingend über das „Eigene“ im Klaren zu sein - Kriegsbemalungen waren in dieser Region beispielsweise kein klassisches kulturelles Element. Bei der Streitfrage, ob finanzielle Entschädigungen, die der Staat sehr wohl leistete, den Verlust des vanua kompensieren können, prallen verschiedene Wert- und Gerechtigkeitsvorstellungen aufeinander. Und eine Frage, ob die Besetzung ein Ausdruck von Egoismus und Habgier einzelner zu Lasten eines Wohlstandes der Gesamtbevölkerung sei, erweitert den Grad der Komplexität um Maßstabsgrundlagen der Beurteilung. Ein kulturhistorisch gewachsenes, durchaus klares Werteverständnis lässt sich in der Gegenwart hier nicht mehr widerspruchsfrei anwenden. Normative Unterscheidungen können nicht einwandfrei getroffen werden: Was ist aus religiös-spiritueller Sicht gut oder böse? Wer ist Freund oder Feind? Juristisch bleibt eine Unterscheidung zwi- 
schen Recht gegen Unrecht unklar. Im Falle des Staudammprojektes schließlich explodierte der brodelnde Konflikt in einem Ausdruck von Gewalt.

Diese Arbeit versteht es nicht als Aufgabe, solch einen Konflikt zu bewerten und Lösungsmöglichkeiten zu unterbreiten. Stattdessen stellt sie ein Plädoyer dafür dar, sich der eigenen Wurzeln und Wertetraditionen bewusst zu bleiben, um eine Basis zu haben, solche Konflikte im Vorfeld zu lösen, bevor sie gewaltsam ausbrechen. Entartet das eigene Selbstverständnis nicht zu einer kulturellen Beliebigkeit, lassen sich Gefahren seitens Entwicklungsdynamiken der Transformation besser erkennen und beeinflussen, sodass dem Ziel einer hohen Lebensqualität bestmöglich nachgegangen werden kann. Das vorherige Fallbeispiel des yavirau zeigt hierbei exemplarisch, wie eine Gemeinde ihre kulturelle Identität und ihr traditionelles Werteverständnis erfolgreich reproduziert.

\subsection{Vamua als Essenz des Kulturellen (Zwischenfazit)}

Im vanua manifestieren sich die Ahnen, über welche die soziokulturelle Identifikation mit dem räumlichen wie sozialen Umfeld stattfindet. Vanua ist die Essenz des Kulturellen. Es beinhaltet die immaterielle, gewachsene Geschichte mit ihren Werten, Normen und Traditionen, Mythen und Legenden; und vanua stellt zugleich die räumliche Materialisierung der Kultur dar.

Liegt der Bedeutungsschwerpunkt auf die soziale Gliederungseinheit, kann der Begriff mit Stamm übersetzt werden, stellt man hingegen die räumlichen, übernatürlichen oder die kulturhistorischen Dimensionen in den Vordergrund, verweist das Wort auf das Land, das Jenseits oder die Tradition. Vanua ist damit ein holistischer Begriff der die Glaubensvorstellungen und Werte fidschianischer Gemeinschaften bündelt. Anhand der Schwierigkeit, den Ausdruck vanua adäquat zu übersetzen zeigt sich eine nicht-westliche kulturelle Denkweise. Die Konstruktion einer Mensch-Natur-Dichotomie ist im Fidschianischen nicht angelegt. Das Verhältnis der Menschen zu ihrer ,,natürlichen“ Umwelt ist daher als sozial oder spirituell zu charakterisieren. Eine Unterteilung in eine menschliche und nichtmenschliche Umwelt erscheint irreführend, da die Menschen über die Ahnen mit ihrer Umwelt in Verbindung stehen.

Da die fidschianische Kultur jedoch seit Jahrhunderten externen Akteuren und ihren Denkweisen ausgesetzt ist, greifbar vor allem in der kolonialen Vergangenheit, transformiert sich die Kultur. So haben parallel ökonomische Vorstellungen vom Land als Ressource Einzug erhalten. Diese unterschiedlichen Betrachtungsweisen können sowohl weitestgehend harmonisch nebeneinander existieren, als auch den Stoff für essentielle Konflikte bilden. So besteht kein Widerspruch, wenn Agrarerzeugnisse einerseits dem reziproken Austausch dienen und als Geschenke gesellschaftliche Zusammenhänge, soziokulturelle Hierarchien, und traditionelle Werteverständnisse reproduzieren, und andererseits marktökonomisch in Wert gesetzt werden. Gerade yaqona kann finanziell angebaut werden, obwohl der Konsum zwingender Bestandteil der vormarktwirtschaftlichen Ahnenverehrung 
ist. Die soziale Praxis gibt hier den entscheidenden Schlüssel, in wie fern es als Ware marktwirtschaftlichen Strukturen unterliegt, oder aber in ritualisierten Handlungsabläufen zeremoniell verarbeitet wird. Über diese Trennung kann die gegensätzliche Handhabung als ein paralleles, zusammenhangloses und damit widerspruchsfreies Nebeneinander konstruiert werden.

Es bestätigt sich der konzeptionelle Ansatz, dass die Kultur der autochthonen Bevölkerung ein Hybrid darstellt, in welchem moderne, dichotome Vorstellungen von Land, Mensch, Natur und Arbeit nicht vorbehaltlos eingegangen sind, bzw. mit Karl Polanyis Worten:

What we call land is an element of nature inextricably interwoven with man's institutions. To isolate it and form a market for it was perhaps the weirdest of all undertakings of our ancestors.

Traditionally, land and labor are not separated; labor forms part of life, land remains part of nature, life and nature form an articulate whole. Land is thus tied up with the organizations of kinship, neighborhood, craft, and creed - with tribe and temple, village, guild, and church. (Polanyi 1944/2001: 187)

Die Hybridität der Kultur zeigt sich an einem gleichzeitigen Nebeneinander verschiedener Denkweisen. Diese kommen in einer lokalspezifisch einzigartigen, jedoch keinesfalls beliebigen Mischung zur Geltung, die der Kultur eine Singularität verleiht. Eine Identifikation und ein Wir-Gefühl können aufrechterhalten bleiben, müssen jedoch beispielsweise in Form eines Neotraditionalismus beständig reproduziert werden. Im folgenden Exkurs werde ich die Reproduktion im Rahmen eines Narrativs über das tabu als einen zentralen Wert der Kultur verdeutlichen.

\subsection{Tabu als lokale Antwort auf den globalen Nachhaltigkeitsdiskurs}

Dem südpazifischen Konzept tabu widmete sich bereits James Cook (1728-1784) ausführlich in seinen Tagebüchern. Anfang des 20. Jahrhunderts griff Sigmund Freud den Begriff auf und erklärte, die „Tabuverbote entbehrten jeder Begründung; sie sind [...] für uns unverständlich" (1913/1991: 48). Die heutige Konnotation des Tabus im Westen als ein strenges Verbot, welches nicht hinterfragt werden dürfe, hat wenig gemein mit der damaligen wie heutigen Begriffsbedeutung im polynesischen Sprachraum (Przyrembel 2011: 9f.). Im Sinne der Transkulturation gedeutet, wurde demnach das tabu-Konzept für westliche Sprach- und Kulturregionen so angepasst, dass es zur Erklärung hiesiger sozio-kultureller Praktiken dient und bereichert so den westlichen Kulturschatz. Als eine erste Annäherung der polynesischen Begriffsverständnisse sollte man tabu jedoch mit „heilig“61 übersetzen. Ursprünglich fand die Begrifflichkeit vor allem im Bereich des Fischfangs Anwendung. Fischgründe wurden temporär zum tabu deklariert, zu einer fang-

${ }^{61}$ Die Bibel, ivola tabu, ist treffenderweise „die heilige Schrift“, nicht das „Buch der Verbote“. 
freien Zone. In einer rein naturwissenschaftlichen Betrachtungsweise regeneriert bzw. vermehrt sich in dieser Zeit der Fischbestand.

Zu diesem Ergebnis kommt man nach traditionellem Wissen ebenfalls, jedoch über eine unterschiedliche Erklärung. Ein tabu wird nach einer Beerdigung in Gedenken an den Verstorbenen oder die Verstorbene verhängt. Stirbt eine hoch angesehene Person wie ein Häuptling, so kann das tabu bis zu 180 Tage dauern. Die Schließung der Fischfangzone einer Gemeinschaft (qoliqoli) ist damit eine Geste zu Ehren der Ahnen. Diese erwidern die Geste in der Fruchtbarmachung des Landes bzw. dem Anreichern der Fischbestände.

Im tabu drückt sich also die spirituelle Verbundenheit der Dorfbewohner zu ihrem vanua aus. Das tabu wird über die spirituelle Dimension zur heiligen Stätte. Heiligtümer zu entweihen stellt eine schwere Sünde dar, die den Zorn der Ahnen heraufbeschworen würde. Ein tabu ist also in erster Linie etwas heiliges, die Bedeutung des streng Verbotenen nur eine daraus indirekt resultierende Komponente. ${ }^{62}$

\subsubsection{Maritime Schutzzone}

Die Insel Gau Island scheint von Umweltverschmutzung und Überfischung gekennzeichnet. So bemerkten die Bewohner der 16 Dorfgemeinschaften Gau Islands über die letzten Jahrzehnte hinweg das Verschwinden mehrerer Fischarten und Seegräser, was auf einen Biodiversitätsverlust hindeutet. Trotz der Einführung moderner Netze, Harpunen und motorisierter Boote, also einer vermeintlichen Verbesserung der Fischfangtechniken hinsichtlich Quantität, nahmen auch die Fangmengen ab. Dies lässt auf einen Rückgang der Fischbestände schließen. Ein Mitglied der Dorfgemeinschaft Malawai promovierte im Bereich nachhaltiger Fischereiwirtschaft (Veitayaki 1995). Ihn bat seine Dorfgemeinschaft auf einem seiner Heimatbesuche um Rat.

Das Konzept von maritimen Schutzzonen (MPA, marine protected area) für nachhaltige Fischereiwirtschaft sieht vor, in einem Wasserraum Fischfang und sonstige störende menschliche Einflüsse zu unterbinden. Je flächenmäßig größer und naturräumlich heterogener die MPA, desto vielversprechender ist eine Erholung und ein Wiederanstieg der Populationen unterschiedlichster Meeresbewohner. Die Meerestiere werden auf Dauer die Schutzzone verlassen und Fischfanggebiete bevölkern, sodass nicht nur die Populationen und Biodiversität innerhalb der MPA zunehmen, sondern - zeitverzögert - auch die Fischfangquoten in angrenzenden Gebieten steigen. Da fidschianische Dorfbewohner Fische direkt an der Küste, auf dem Korallenriff als auch vor dem äußeren Riff fangen, jedoch in der Regel keine Hochseefischerei betreiben, bedeutet dies einen möglichst breiten

\footnotetext{
${ }^{62}$ Die Grundidee des tabu besteht nicht nur beim Fischfang. So können auch ländliche Orte oder Tier- und Pflanzenarten heilig sein. In diesen Fällen gilt das tabu oftmals permanent, kann jedoch im Rahmen von yaqona-Zeremonien unter Leitung des Häuptlings punktuell aufgehoben werden: So können heilige Stätten wie Friedhöfe betreten (siehe Kap. 7.2.6) oder einzelne Bäume gefällt werden.
} 
Streifen von der Küste bis zum äußeren Riff zu schließen, der am besten Flussläufe und Mangrovenwälder beinhaltet.

Während hinter einer MPA ein hochgradig wissenschaftliches Konzept steckt und es zur Optimierung einer MPA gerade bezüglich der naturräumlichen Heterogenität Expertenwissen bedarf (Brown et al. 2001: 417-420; Jones 2002: 199201), kann das Konzept zur praktischen Anwendung auf lokales Wissen und Management-Vermögen heruntergebrochen werden. Solch eine von der Gemeinschaft weitestgehend autonom und lokal gemanagte maritime Schutzzone (FLMMA, Fijian locally managed marine area) beschloss die Dorfgemeinschaft auf einer Dorfgemeindeversammlung (Govan et al. 2009; Veitayaki 2006). Namentlich wurde die Schutzzone jedoch nicht mit FLMMA benannt, sondern als tabu-area beschrieben.

Als tabu verstanden ist das Konzept nicht nur vereinbar mit dem traditionellen Werteverständnis, sondern verfestigt gar die kulturelle Wertebasis. Da es zudem im Rahmen der fidschianischen Etikette von einer anerkannten Persönlichkeit vorgeschlagen wurde, stieß die tabu-area auf breite Akzeptanz. Um den Wirkungsgrad der Schutzzone zu erhöhen, errichtete Malawai gemeinsam mit dem Nachbardorf Lamiti eine gemeinsame Schutzzone, die die qoliqoli beider Dörfer überlappt. Das im Jahr 2000 markierte tabu erstreckt sich von der Schneise des Malawai Rivers zum Lamiti River und von der Küste zum äußeren Riff. Dabei wird die vorgelagerte Insel Dakui umschlossen (siehe Karte 2). Aufgrund des Wissens um den Nutzen und der Heiligkeit des tabu, sowie sozialer Kontrollmöglichkeiten in fidschianischen Dorfgemeinschaften wurde nicht gewildert. Nach wenigen Monaten bemerkten die Fischer bereits Zuläufe von Fischen in den benachbarten Gebieten. Der schnelle Erfolg zementierte die hohe Akzeptanz. Nach - gerechnet nach meinem letzten Besuch - nunmehr 13 Jahren permanenter Schließung entdecken die Bewohner zudem Arten wieder, die seit Jahrzehnten nicht mehr gesichtet wurden. ${ }^{63}$

Die FLMMA stellt nicht nur in ihrem Kernanliegen, die maritimen Ressourcen und Biodiversität zu schützen und nachhaltiges Fischen zu fördern einen Erfolg dar. Zusätzlich - dorfübergreifend angelegt - schweißt es über die gemeinsame Betreuung die beiden Dörfer Malawai und Lamiti zusammen, die sich bereits zuvor schon durch intensive soziale Interaktionen gegenseitig positiv beeinflussten. So wird gemeinsam eine Grundschule gemanagt, bei Festivitäten lädt man sich gegenseitig ein und in schwierigen, arbeitsintensiven Situationen - wie beispielsweise die Veranstaltung einer Beerdigung - hilft man sich gegenseitig aus.

\subsubsection{Gefahr einer Entzauberung der Welt}

Der Fischbestände sind im tabu wesentlich beeindruckender als in den Fanggebieten außerhalb. Eine Plünderung des tabu stellt daher eine große Versuchung dar,

${ }^{63}$ Während die junge Generation für sie neue Fischarten entdeckt, sprechen die Älteren von einer Rückkehr bekannter Fischarten. 
wird jedoch nur äußerst selten festgestellt. Ein naturwissenschaftlich fundiertes Wissen um den nachhaltigen Nutzen einer FLMMA reicht nicht zwingend aus, der Versuchung zu widerstehen. Weiterhin braucht es einer intakten Gemeinde, der man wohlgesonnen ist und deren Mitgliedern man ein enormes Vertrauen entgegenbringt, damit man die Gemeinschaftsressource nicht auf Kosten der anderen ausraubt. Im Falle von Plünderungen werden die Taten daher außerhalb der Gemeinschaft verortet, um den Frieden zu wahren. So werden die Plünderungen unkonkret Nachbargemeinden zugeschrieben - was meines Wissens bisher jedoch nicht zu Verstimmungen führte - oder Fischereiflotten - was aus Effizienz- und Logistikgründen jedoch unwahrscheinlich ist. Die Täter bleiben daher unentdeckt. Ein weiterer, entscheidender Erklärungsgrund für die jahrelange Selbstrestriktion liegt im Glauben. Die spirituelle Aufwertung der FLMMA zum heiligen und damit einzigartigen und unendlich wertvollen tabu stellt einen Grundpfeiler für den Erfolg dar. Eine naturwissenschaftlich-marktwirtschaftliche NachhaltigkeitsArgumentation könnte dies nicht leisten.

Angetan vom Erfolg der tabu-area suchten weitere Dörfer Gau Islands Rat. Seit 2007 hat jedes Dorf Gau Islands ein tabu. Mit Unterstützung der USP und internationaler Hilfsorganisationen kooperieren inzwischen alle 16 Dörfer unter der eigens erschaffenen Dachorganisation „Lomani Gau“ (Sich um Gau Island sorgen) und koordinieren ihre Entwicklungsbestrebungen. Neben weiteren Anpassungsmaßnahmen im Sinne nachhaltiger Fischereiwirtschaft widmet man sich ebenso einer nachhaltigen Feldwirtschaft, dem Schutz des Regenwaldes, einer Umkehrung des Entwaldungsprozesses, sowie dem Küstenschutz und einer Anpassung an Naturgefahren und den Auswirkungen des Klimawandels. Die Inselbewohner befinden sich auf dem Weg zu einem ganzheitlichen ökologischen Ressourcenmanagement des gesamten Ökosystems Gau Islands von den Berggipfeln bis zu den äußeren Riffen. Die dahinterliegende Motivation ist jedoch nicht nur ökonomisch, sondern ebenso sozio-spirituell. Aufgezogen werden die Anpassungsmaßnahmen mit dem Kerngedanken, die Qualität des vanua zu steigern. Dass die Ahnen dies würdigen, sodass diese einen sozio-ökonomisch besser stellen, ist ein indirekter, positiver, ,natürlicher" ${ }^{\text {“64 }}$ Begleiteffekt.

Eine kulturell nicht angepasste Promotion eines nachhaltigen Managements ökologischer Ressourcen würde die spirituelle Motivation untergraben. Eine Entzauberung des vanua als Reduktion auf physisch-materielle Dimensionen würde ihren Wert mindern. Die spirituelle Dimension ist jedoch die treibende Kraft, die den anhaltenden Erfolg der Maßnahmen erklärt. Ihr Wegfall würde in doppelter Hinsicht Einschnitte in die Lebensqualität bedeuten, da man erstens mit der Entzauberung der Umwelt als Ressource sie ihrer Einzigartigkeit und Heiligkeit beraubt, was wiederum die Motivation, diese zu bewahren lindern würde, worunter zweitens die Qualität des Ökosystems leiden würde.

${ }^{64}$ „Natürlich“ im Sinne des kulturellen Werteverständnisses, nachdem die Ahnen einem grundsätzlich wohlgesonnen sind. 


\section{Lebensqualität in fidschianischen Küstendörfern}

In diesem Kapitel soll explizit das kulturelle Wertesystem erarbeitet werden, aus welchem sich Maßstäbe hoher Lebensqualität ableiten lassen. Bisher wurde induktiv von einzelnen Elementen der fidschianischen Kultur auf allgemeine Werte und indirekt auf Lebensqualität geschlossen. Vervollständigt und gegengeprüft werden soll diese Methode nun über eine deduktive Betrachtungsweise. In verschiedensten Kontexten wurde mir von den Dorfbewohnern folgender Dreiklang als Ideal präsentiert, was Lebensqualität ausmache: spirituelles, mentales und physisches Wohlbefinden. Ähnlich formuliert zeichne sich ein sinnhaftes, gutes Leben im Dreiklang spiritueller, physischer und mentaler Fitness aus (siehe Abb. 13 und Abschlussdiskussionen in Malawai und Dravuni).

In einem ersten Schritt wird der Dreiklang normativ sowohl in individueller als auch struktureller Hinsicht durchleuchtet um die Basis hoher Lebensqualität zu erfassen. Auf dieser Grundlage können anschließend Praktiken zu ihrer Erzeugung und Bewahrung analysiert werden. Hierfür stelle ich zunächst vor, wie im Zuge der Chancen und Risiken der gesellschaftlichen Transformation der vielleicht wichtigste kulturelle Brauch der iTaukei zur menschlichen Sicherung, kereke$r e$, ausgelebt werden kann. Anschließend gehe ich auf Möglichkeiten der Überwindung von Verwundbarkeiten gegenüber Naturgefahren und Klimawandel der hybriden Kultur ein. Denn insbesondere im kulturellen Vermögen, mit Extremsituationen umzugehen, zeigt sich die Qualität der sozialen Sicherung. Mithilfe einer Bewertung, wie tauglich die geschilderten Handlungspraktiken zur Erreichung des Dreiklangs sind, kann auf die Lebensqualität in fidschianischen Dorfgemeinschaften insgesamt geschlossen werden.

8.1 Einklang im Dreiklang - mentale, physische und spirituelle Fitness als Quelle guten Lebens

Ein sinnerfülltes Leben kennzeichnet das Streben nach geistiger, körperlicher und spiritueller Fitness. Diese stehen in Wechselwirkung zueinander. Grundlage des Spirituellen ist der Glaube. Eine Lebensführung nach Gottes Wille kann dabei nicht auf explizit religiöse Praktiken beschränkt sein, sondern jeder individuellen Handlung sollten die über die Religion vermittelten Werte zugrunde liegen. Seinen Glauben lebt man also nicht (ausschließlich) in der Kirche aus, sondern im sozialen Miteinander (Farmer, ehem. Bankangestellter, 37, Dravuni). Geistig gesund ist, wer die religiös fundierten Werte im sozialen Miteinander auslebt. Zur Erstarkung der geistigen Fähigkeiten von Kindern und Jugendlichen dient die Erziehung bzw. Wertevermittlung in Schule, Kirche und Familie. Parallel benötigt es zur Ausübung eines geistig-spirituell erfüllten Lebens einer körperlichen Gesundheit, sodass physische Fitness ein hohes Ideal darstellt.

An keines dieser Kriterien wird jedoch ein Mindestmaß gesetzt, sodass man altersschwachen sowie körperlich oder geistig behinderten Menschen ihren Lebens- 
sinn nicht abspricht. Sofern Menschen nach diesem Dreiklang streben, stellen sie eine Bereicherung für die Gemeinschaft insgesamt dar. In Natavea kam ich in Kontakt zu einem geistig Behinderten ${ }^{65}$, sowie in Vatulele in Kontakt zu geistig wie körperlich Behinderten, die bestens in ihren Gemeinschaften integriert waren. Dies zeigte sich nicht nur daran, dass sie am gesellschaftlichen Leben teilnahmen, seien es Zugehörigkeiten zu dörflichen und kirchlichen Gruppen oder der Besuch von Fundraising-Partys, Kirchgängen, Hochzeiten oder Beerdigungen. Sie bekamen zudem auch keine unnötigen Sonderbehandlungen und wurden bei gesellschaftlichem Fehlverhalten wie Gier oder Müßigkeit mit Tadel bedacht. So waren sie genauso wenig wie andere vor Scherzen gefeit, die in der fidschianischen Gesellschaft mitunter recht derbe ausfallen können. Das spielerisch-humoristische Miteinander lockert die Dorfgemeinschaften auf, trägt zur Integration bei und reproduziert indirekt Werte.

\subsubsection{Projektion des Dreiklangs guten Lebens in gesellschaftlichem Oberbau}

Während auf individueller Ebene das Streben nach mentaler, physischer und spiritueller Fitness als Kern eines ausgefüllten Lebens ausgemacht werden kann, stehen hierfür auf der strukturellen Ebene drei gesellschaftliche Institutionen: Religion, Häuptlingswesen und die soziale Gemeinschaft. Im fidschianischen stehen hierfür die Begriffe lotu, matanitu und vanua (Newland 2009: 189). Auch diese drei Begriffe dürfen nicht als losgelöst von einander betrachtet werden. Alleine der Begriff vanua kann schon nicht einzig für „Gemeinschaft" stehen, in ihm schwingen zwingend Vorstellungen von Land mit, welche ihrerseits nicht bloß physischmateriell räumlich, sondern ebenso sozialräumlich als auch spirituell unterlegt sind (siehe Kap. 7).

Ebenso beinhaltet matanitu eine göttliche Ordnung auf Basis des vanua. Das Häuptlingswesen kann also nicht abgekoppelt der anderen beiden Institutionen betrachtet werden und vereinigt ebenfalls alle drei Dimensionen guten Lebens. Irreführend beansprucht das Staatswesen ebenfalls den Begriff matanitu für sich. Als Parallelstruktur wahrgenommen fehlt der Regierung jedoch die Identifikation und Legitimation, während der Dreiklang aus lotu, Häuptlingswesen und vanua das Selbstverständnis prägt und sinnstiftende Funktionen beinhaltet.

Aus der religiös-spirituellen Sphäre entstammt der normative Unterbau, menschliche Handlungen und Strukturen als sinnhaft charakterisieren zu können. Ohne lotu ist das menschliche Leben demnach sinnfrei. Spiritualität begrenzt sich nicht auf religiöse Praktiken rund um die Kirche, sondern sollte als normatives Fundament in jeder menschlichen Handlung bewusst eingebettet sein. „Gemeinschaft" ist entsprechend ein normatives und damit spirituelles Gut; Die Ausdrücke „soziales Zusammenleben“ oder „Zwischenmenschlichkeit" sind normativ belegt. Die Wertigkeit wird spirituell begründet. Das Ideal eines „Miteinanders“, von

\footnotetext{
${ }^{65}$ Sowohl nach meinem eigenen Verständnis von „Behinderung“, als auch nach dem der Dorfbe-
} wohner. 
„Partizipation“, „Kooperation“ oder „Empathie“ entspringt einer Göttlichkeit bzw. Heiligkeit. ${ }^{66}$

Vanua ist demnach ohne lotu nicht zu verstehen, umgekehrt kann lotu nicht losgelöst von der sozialen Welt praktiziert werden. Der Versuch einer Hierarchisierung der Begrifflichkeiten lotu, matanitu und vanua ist irreführend, da sie nicht ohne einander gedacht werden können. So wurde mir bei einem Venn-Diagramm in Dravuni (siehe Abb. 13) erläutert, dass man nicht mit unterschiedlichen Kreisgrößen arbeiten wolle. In diesem Dreiklang könnte lotu allenfalls als primus inter pares ausgemacht werden. Es wurde zudem bewusst von true church gesprochen, um sich von der Kirche als weltlicher Institution abzugrenzen.
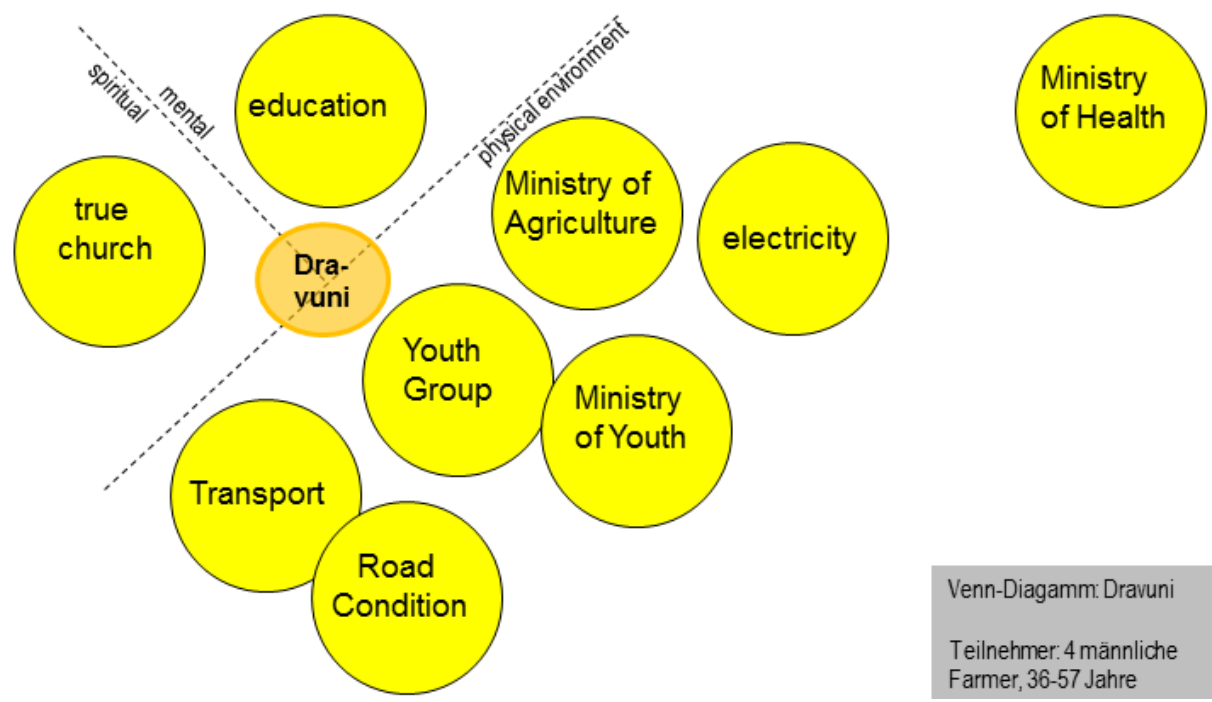

Abb. 13: Venn-Diagramm in Dravuni (Visualisierung vom Autor auf Grundlage der Diskussion mit den Teilnehmern um die Ordnungsfelder „mental“, „spiritual“ und „physical environment" erweitert)

\subsubsection{Intergenerationelle Dimensionen eines guten Lebens}

Die individuellen Bestrebungen und nach spiritueller, mentaler und physischer Fitness und die gesellschaftlichen Institutionen ergänzen sich. Eine ideale Umsetzung der individuellen Bedürfnisbefriedigung reproduziert den gesellschaftlichen Oberbau. Der Einzelne steht in Wechselbeziehung zu seinen Mitmenschen. Nur im Zusammenspiel können die eigenen Qualitäten ausgebildet werden. Ohne Mitgefühl leidet man unter mentalen Störungen, physische Leistungsfähigkeiten

\footnotetext{
${ }^{66} \mathrm{Ob}$ die heutigen Ansichten in einer christlich-abendländlichen Beeinflussung begründet liegen, oder aber auf vorkolonialen Wertesystemen basieren, lässt sich im Rahmen dieser Arbeit nicht klären.
} 
erfahren einen Sinn nur in ihrer Anwendung für die Gemeinschaft. Deren Fortbestand sichern die nachfolgenden Generationen. Dies erklärt folgenden Ausspruch nach der idealen Gestaltung des Lebens:

Alles, was man tut, dient dem eigenen mentalen, physischen und spirituellen Woblbefin-

den. Damit ist letztlich alles, was man tut, für die Jugend. (Farmer, Dravuni, 57)

Nach einer solchen Lebensauffassung existiert kein Widerspruch zwischen individuellen und kollektiven Lebensweisen. Jede „gute Tat" für die Gemeinde ist zugleich eine Stärkung der eigenen Lebensqualität und einen Egoismus kann man nur über die Förderung des Allgemeinwohls befriedigen. Ein „Aufopfern“ für die Gemeinde wiederum ist paradox; jedes eigene Leid und jede eigene Schwächung belastet die Gemeinde. Dieses Narrativ vom gesunden Leben ist dabei selbstverständlich ein idealisierter Wunschzustand. Er sollte nicht über wahrgenommene Realitäten hinwegtäuschen, in denen Menschen gegensätzlich von Versuchungen, Krankheiten und schwarzer Magie heimgesucht werden.

\subsubsection{Störungen eines guten Lebens aufgrund gesellschaftlicher Transformation}

Die vorgestellten Ideale weisen Charakteristika auf, bei denen sich eine Prüfung auf Universalität lohnen könnte - ggfs. erklärbar durch die hybride Kultur und verschärft durch eine Darstellung in westlicher Sprache. Westliche Institutionen sowie abendländliche Theoriegebäude und Gedankenkonstrukte finden hier durchaus Ansatzpunkte, seien es Freiheit, Brüderlichkeit, Nachhaltigkeit, soziale Gerechtigkeit oder ähnliche Narrative. Auf Fidschi nehmen sie in ihrer Anwendung jedoch eine kulturell einzigartige Form an und westliche Einflüsse torpedieren mitunter das als in sich schlüssige, ganzheitlich wahrgenommene Verständnis von Lebensqualität.

Sind lotu, matanitu und vanua unumstrittene Ideale, mögen kritische Auffassungen von Kirche, Staat und Oberster Häuptlingsrat - vom Westen geschaffene Gebilde - widersinnig erscheinen. Sie als elitäre Institutionen verstehen zu können, die ein Eigenleben zur Erfüllung von Partikularinteressen entwickeln können, lässt sich nur schwer in die kulturelle Werte- und Wissensbasis aufnehmen. Der Umstand, dass sprachlich keine Differenzierung stattgefunden hat, der Staat mit matanitu und die Kirche mit lotu übersetzt werden, verkompliziert die Widersprüchlichkeit. Diffus und verstörend können demnach Wünsche der Militärregierung wirken, die politische Macht der Kirche und des GCC zurückzudrängen (siehe Kap. 5.2.3).

Zusammenfassend kennzeichnet die fidschianische Gesellschaft eine hybride Kultur, die ein festes (neo-)traditionelles Wertefundament kennzeichnet, welches Möglichkeiten zur Erreichung einer hohen Lebensqualität aufzeigt und Identität stiftet. Unsicherheit besteht allerdings, inwiefern der anhaltende, tiefgreifende gesellschaftliche Wandel diese Stabilität gefährdet, da sich im Zuge der Transformation verschiedene Weltanschauungen zusammenfinden müssen. Dies geschieht 
nicht konfliktfrei. Aufgrund der festen Wertebasis fidschianischer Gemeinschaften können die Konflikte jedoch weitestgehend gewaltfrei ausgetragen werden. Zufriedenstellende Lösungen werden jedoch nicht immer gefunden.

\subsection{Kerekere - Geben und Nehmen}

Kerekere steht für eine Bitte, die man nicht abschlagen darf. Dahinter verbirgt sich ein Austauschsystem, das eine zentrale Stellung in der fidschianischen Kultur einnimmt (Nayacakalou 1978: 112f.; Taylor 2005: 117f.). Hierbei werden zumeist Waren, gelegentlich aber auch Dienstleistungen erfragt. Sofern Bedürftige bei Besitzenden fragen, stellt diese Alltagspraxis ein soziales Sicherungssystem dar und wird als gerechtes Prinzip sozialer Umverteilung angesehen. Kerekere hat dabei reziproken Charakter, da prinzipiell alle Menschen im Rahmen ihrer Möglichkeiten Geber sind.

\subsubsection{Mythos einer einseitigen Ausnutzung aufgrund gesellschaftlicher Transformation}

Bei der praktischen Anwendung führt kerekere oftmals zu Verstimmungen; so lautet in Gesprächen gerne der Tenor, dass der Brauch dreist überbeansprucht wird und anfällig gegenüber bestimmten Menschen ist, welche andere, beispielsweise einen selbst, ausnutzen wollen. In der Literatur werden diesbezügliche Konflikte gerne als Spannung im Zuge eines gesellschaftlichen Wandels gedeutet. Aufgrund einer Immobilität der Dorfbewohner früherer Zeiten könne niemand das System ausbeuten, da man Gegenleistungen nicht entfliehen könne. So findet Taylor (2005: 117) Gründe, warum kerekere heutzutage nicht mehr funktioniere:

(i) people are not stationary enough to guarantee that favors that they have done for the people will be returned' over time, and (ii) the value of the favors is radically different if families live dispersed between urban and rural environments. So whilst a neighbor in the village may lend a kerosene lamp, this does not equate to looking after that same neighbor's children in the city, feeding, clothing and sending them to school for the year.

In mehrfach praktizierten partizipativen Gruppenmethoden habe ich daher kerekere untersucht, um im Wandel des kerekere Systems das Spannungsfeld gesellschaftlicher Transformation wie im Brennglas zu analysieren. Dank der Offenheit der Methode bin ich jedoch zu der Erkenntnis gekommen, dass die zitierte Darstellungsweise zu verkürzt und schlussendlich eine Fehlinterpretation ist.

Selbst ohne zusätzliche Empirie lässt sich die Unstimmigkeit bereits anhand ihrer simplizistischen Annahmen offenlegen. Dass kerekere in der präkolonialen Ära oder den kolonialen Anfängen nicht anfällig für Vorteilnahmen gewesen sei, ist empirisch nicht zu belegen. Als negative Form von kerekere wird beispielsweise kerekere raica empfunden (siehe Abb. 14 \& 15), etwas „auf Sicht“ bzw. bei Gelegenheit und nicht bei Bedürftigkeit zu verlangen. Es erfordert nicht viel Phantasie, sich Möglichkeiten auszudenken, wie man auch ohne höhere Mobilität das System zum eigenen Vorteil ausnutzen könne, sofern man nur wollte. Ein Unterbinden 
würde eine derart unfreie vormoderne Gesellschaft voraussetzen, wie sie nur in totalitären Systemen vorkäme - welche jedoch die technischen Kontrollmöglichkeiten der Moderne benötigt hätten.

Weiterhin wird der Grundgedanke reziproker Austauschbeziehungen zu vereinfacht und damit irreführend dargestellt. Wie schon von Mauss für pazifische Kulturen erörtert (siehe Kap. 2), zirkulieren Gaben, sodass kein Anspruch auf Gegenleistungen seitens der beschenkten Person besteht. Dies ist ja eine Stärke der Gabe, dass sie als Geschenk aufgenommen wird. Ebenso werden das intergenerationelle Moment der Gabe und das Motiv des sozialen Ausgleichs außer Acht gelassen. Die Möglichkeit eines Fortzugs behindert daher erstens keine reziproken Austauschbeziehungen, zumal zweitens aufgrund der intensiven Interaktionen zwischen der Stadt- und Landbevölkerung (siehe Kap. 5.4 \& 6.5) Mobilität keinen Abbruch sozialer Beziehungen bedeutet. Im Gegenteil deutet die räumliche Ausweitung des Brauches auf eine qualitative Stärkung des Sicherungspotentials hin. Hier widerspricht sich Taylor deutlich, da nochmals explizit auf diese Interaktionen eingegangen wird, um Imbalancen in der Wertigkeit jeweiliger Gaben herauszuarbeiten. Dabei das Verleihen einer Petroleumlampe einer einjährigen Komplettversorgung von Schulkindern als typische ländliche bzw. städtische Gaben gegenüber zu stellen, ist reine Polemik. Gaben sind reziprok und damit gleichwertig, wenn auch nicht zwingend in Form von Preisen gemessen. Dorfbewohner übernehmen Pflichten und Aufgaben der Auswertigen in der Dorfgemeinschaft, was ihnen hohe Dankbarkeit und Anerkennung bringt. Kerekere zielt nicht auf Tausch sondern soziale Umverteilung ab; sofern manche Personen oder Personengruppen mehr geben als nehmen, liegt das zunächst in ihrer Situiertheit begründet.

\subsubsection{Anpassungsfähigkeit Dank öffentlicher Diskussion}

Damit kerekere nicht ausgenutzt und überstrapaziert wird, bedarf es unabhängig eines Wandels gesellschaftlicher Strukturen fortlaufender Diskussion innerhalb der Gemeinschaften, um normative Chancen und Grenzen dieser kulturellen Praxis beständig (re)produzieren zu können. So habe ich in der Methodenanwendung nach Beispielen guter und schlechter Handhabung gefragt (siehe Abb. 14). Auf Basis der Beispiele wollte ich die Diskussionsteilnehmer in einem zweiten Schritt ein Gesamturteil über den Brauch fällen lassen, um auf seine gesellschaftliche Akzeptanz zu schließen. Eine Aufteilung in jüngere und ältere Diskussionsgruppen könne dabei möglicherweise Rückschlüsse auf intergenerationelle Verschiebungen erlauben - so meine Anfangsintention. Die Frage nach einem Pauschalurteil erwies sich dabei als unklug. ${ }^{67}$ Es gibt sowohl normativ richtige Formen von kerekere, welche es beizubehalten gilt, als auch verwerfliche. Über jedes der genannten Beispiele - welche in der Diskussion kontextualisiert wurden - herrschte

\footnotetext{
${ }^{67}$ Als Vergleich: Eine Frage nach einem Pauschalurteil, ob Süßigkeiten gut oder schlecht seien, benötigt einen spezifizierten Kontext zur Beantwortung.
} 
generationenübergreifend Einstimmigkeit über die Einordnung. In einer weiteren Ausführung wurde daher allgemeiner nach Vor- und Nachteilen von kerekere gefragt, um expliziter kulturelle Wertvorstellungen erfassen zu können (siehe Abb. 15).

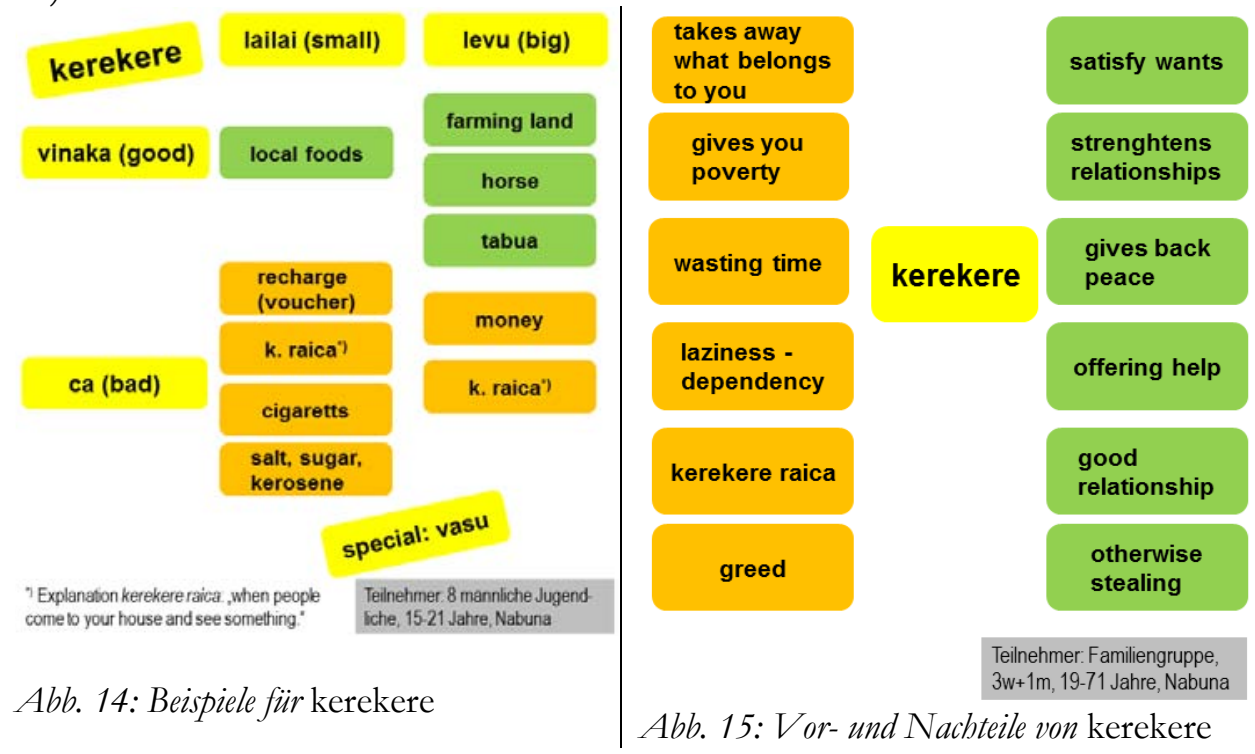

Die Ideale des guten kerekere (kerekere vinaka) werden intergenerationell geteilt und als kulturelles Gut zur Stärkung der eigenen Identität gepriesen. Dass schlechte Formen (kerekere ca) existieren, liegt in der Anfälligkeit der Menschen begründet, sich wider besseren Wissens vermeintlich einfacheren, „falschen“ Lebensentwürfen hinzugeben. Oder, wie die Literatur über Sozialkapital abseits spiritueller Wertkonnotationen schreibt, besteht in sozialen Beziehungssystemen die latente Gefahr einer Verkehrung ins unsoziale (siehe Kap. 2.4). Basierend auf Feldforschung im Jahre 1954 beschreibt bereits Rusiate Nayacakalou in seiner posthum veröffentlichten Studie (1978: 137), wie kerekere als Möglichkeit genutzt wird, Güter und Dienstleistungen unentgeltlich zu beanspruchen - dadurch jedoch mitunter Beziehungen belastet werden. Den Brauch deswegen nicht aufzugeben, sondern kerekere ca auszuhalten, drückt die Stärke des Sozialen einer Gemeinschaft aus. Öffentliche Diskussion und Meinungsbildung sowie damit einhergehende soziale Kontrolle sorgen für Abschreckung, minimieren negativen Formen und machen den Brauch gesellschaftskonform. In der öffentlichen Verunglimpfung von kerekere ca sieht Nayacakalou (1978: 137f.) einen Trend, der die Geldwirtschaft als Gegenmodell zur Gabe stärkt. Seiner Meinung nach werden traditionelle Beziehungsmuster jedoch erhalten bleiben, sodass sich ein dualistisches Beziehungssystem entwickeln könne: während man bei Geldgeschäften unpersönliche Haltungen annehmen werde, bleibe bei anderen Angelegenheiten das persönliche 
Moment erhalten. Diese Vorausschau kann ich 60 Jahre später bestätigen anhand der Erfahrungen mit kerekere und dem monetären wie parallel dazu nichtmonetären Umgang mit yaqona (siehe Kap. 7.4) bestätigen.

Anhand mancher Beispiele, die für kerekere genannt werden (Zigaretten, Benzin, Aufladeguthaben für Mobiltelefone), lässt sich zwar erkennen, dass die Gesellschaft sich wandelt. Solange der Brauch in seinen als zentral empfundenen Elementen jedoch fortbesteht, ist dies ein Anzeichen, dass das kulturelle Wertefundament intakt bleibt und das traditionelle Sicherungssystem in seiner Qualität nicht bröckelt, sondern sich räumlich und qualitativ an den Wandel anpasst.

Um die kulturelle Identität wahren zu wollen, ist auch dieser Brauch neotraditionell aufgeladen. Gegenstände, die einen hohen symbolischen Wert besitzen, sind tendenziell gutem kerekere zugeordnet, wohin gegen Sachen, deren Wert in erster Linie monetär festgehalten wird, negativ konnotiert sind. Marktwirtschaftliche, monetäre Geschäftsbeziehungen sind unpersönlich, weshalb soziale Netzwerke diesem Werteverständnis nach nicht zur individuellen Geldbeschaffung genutzt werden sollen. Ein tabua, Walzahn, ist in der Kultur die symbolisch hochwertigste Gabe (Nayacakalou 1978: 113; Ravuvu 1987: 22-25) und wird beispielsweise den Schwiegereltern in spe bei der Anfrage um die Hand ihrer Tochter dargereicht. Um an einen solchen Zahn zu gelangen, soll der Bräutigam gerne mittels kerekere bei Älteren, beispielsweise dem talatala nachfragen, ,and not to buy a tabua from the Indian in town" (Priester, 36, Nabuna). ${ }^{68}$ Dieses Zitat enthält eine dreifache Abgrenzung zur Bestimmung der eigenen kulturellen Identität, erstens von den Indo-Fidschianern, zweitens von städtischen Milieus und drittens von monetären Beziehungen.

Auch das Beispiel des Pferdes (siehe Abb. 14) bestätigt den „neo“-Charakter des Traditionalismus. Pferde wurden von den Europäern eingeführt, werden auf Koro Island aber seit vielen Jahrzehnten zum Transport in der Landwirtschaft gehalten. Einzelne Pferde sind ausgerissen und verwildern. Inzwischen sind Pferde im kulturellen Landschaftsbild Koro Islands verinnerlicht. Im vorliegenden Fall wurde über eine Heiratsbeziehung die angeheiratete Verwandtschaft eines Nachbardorfes nach einem Pferd gefragt. Ein angesehener Familienangehöriger reiste in jenes Dorf und trug im Rahmen einer Zeremonie und unterfüttert mit Gastgeschenken sein Anliegen vor, für seinen Neffen ein Pferd zu erbitten. Dieser besitze kein eigenes Pferd, wolle jedoch seine Farmarbeit intensivieren, da er eine junge Familie zu ernähren habe. Der gewählte Kommunikationsweg über einen Fürsprecher ist kulturell hoch angesehen, wie ich bereits bei meinem sevusevn (siehe Exkurs 1) aufzeigte. Über die sozio-kulturelle Rahmung erhielt dieses kerekere ein traditionelles Fundament, das Pferd wurde an seinem symbolischen und nicht an

\footnotetext{
${ }^{68}$ Die Darbietung eines tabua findet immer in zeremoniellem Rahmen statt. Zur Erhöhung der Symbolkraft des tabua wird bei der Übergabe - soweit bekannt - dessen Geschichte nacherzählt, zu welchen bedeutenden Anlässen exakt dieser tabua bereits gedient habe. So wird dem Zahn mittels seiner einzigartigen Sozio-Historie eine Seele eingehaucht, welche die Symbolkraft (re)produziert.
} 
seinem monetären Wert gemessen. Dies schuf gesellschaftliche Akzeptanz und festigte die guten Beziehungen zwischen den benachbarten Familien.

Die Praxis des kerekere zeigt, wie anpassungsfähig kulturelle Bräuche an gesellschaftliche Transformationsprozesse sein können. Entgegen der gängigen Literatur konnte ich Dank des offenen, partizipativen Methodenzugangs feststellen, dass der Brauch den gesellschaftlichen Wandel mitgeht, um so die Chancen einer räumlichen Ausweitung und damit einhergehenden qualitativen Aufwertung sozialer Sicherung zu adaptieren, welche die Transformation mit sich bringt. Der Brauch benötigt seit jeher einer fortlaufenden öffentlichen Diskussion, um Ausartungen vorzubeugen. Da sich diese im Zuge der Transformation keineswegs reduzierte, trägt dieses Instrument zur menschlichen Sicherung bis heute wesentlich zur Reproduktion des kulturellen Wertefundaments bei und erzeugt Lebensqualität.

\subsection{Klimawandelanpassung und Naturgefahrenvorsorge}

In den fidschianischen Medien ist der Klimawandel omnipräsent. Vertreter Fidschis haben eine nationale Strategie zur Klimawandelanpassung erarbeitet und sitzen in vielen internationalen Gremien. Fidschianer repräsentieren aktuell die SIDS in drei Klimawandelbezogenen UN-Institutionen (Fiji Ministry of Foreign Affairs Media Release \& SPREP 2014). Hohe Regierungsbeamte fliegen von einem Klimagipfel zum nächsten um bestens auf die möglichen Gefahren vorbereitet zu sein. Dem Thema wird so viel Beachtung geschenkt, dass das Thema „Klimawandel" verpflichtend in das Curriculum der Grund- und weiterführenden Schulen aufgenommen wurde (Fiji Ministry of Foreign Affairs Media Release 2012; SPC 2014a).

Entsprechend internationaler Agenden wird der Diskurs über den Klimawandel in Forschung und Anwendung dabei häufig in Beziehung zum Diskurs über Naturgefahren gesetzt. Gerade aus der Schnittstelle zwischen Klimawandelanpassung und Reduzierung von Naturgefahren erhofft man sich Synergien (Birkmann \& Teichman 2009: 6-14; Gero et al. 2010: 8). Für Fidschi ist hierbei insbesondere die Handhabung tropischer Zyklone von Bedeutung (siehe Kap. 5.3).

\subsubsection{Herausforderungen bei der Andockung neuer Wissenssysteme an traditionelle Basen}

Aufgrund der Tiefe des Themas „Klimawandel“ und der Breite des Personenspektrums, das zu diesem Thema ohne größere Vorschulung arbeitet (SPC 2014b), mangelt es an Expertise in den Medien, im Schulunterricht sowie in Alltagsgesprächen. Der Klimawandel wird gerne einseitig beleuchtet und Zusammenhänge äußerst vereinfachend dargestellt. Insbesondere über Maßstabsebenen wird nicht kritisch reflektiert, sodass das vorhandene Wissen über Ursachen und Auswirkungen des Klimawandels in lokalen Kontexten mitunter falsch angewendet wird. So klagt man in den Untersuchungsdörfern, für akute Wetterlagen sei man eigenverantwortlich; Trockenperioden und Starkregen werden mit dem Fällen einzelner Bäume erklärt. Für jede Wetterschwankung hat man nun eine Universalerklärung. 
Auf Basis des neuen Wissens wird mir in Dravuni erklärt, da am Flussoberlauf Bäume gefällt würden, steige bei ihnen der Meeresspiegel. Solche Fehlinterpretationen strapazieren Beziehungen zu Nachbardörfern. Fatal sind ebenso Umkehrschlüsse, mittels lokaler Aufforstungsprojekte ließe sich der Klimawandel stoppen, würde der Meeresspiegel wieder sinken. So bringt das diffuse Wissen über den Klimawandel keinen Mehrwert, der bei Entscheidungen hilft, sondern sorgt für Fehlentwicklungen und unnötige Belastungen sozialer Beziehungen. Ähnlich registrieren auch Lata \& Nunn bei ihrer Studie im Rewa-Delta Misskonzeptionen bezüglich räumlicher und zeitlicher Wirkungsweisen des Klimawandels (2011: 180f.)

Traditionelles, implizites Wissen über ökologische Zusammenhänge wird vom Klimawandel-Diskurs beeinflusst. Es existiert traditionelles Wissen um eine Vielzahl an Anzeichen, einen Zyklon vorherzusagen. Langfristige Indikatoren, die Monate im Voraus auf einen Zyklon hindeuten, wären, dass der Brotfruchtbaum (Artocarpus altilis) im Vorfeld außergewöhnlich viele Früchte produziert. Der Mangobaum ist saisonal und seine Früchte sind während der Zyklon-Saison reif in den Jahren eines Zyklons setzt der Reifungsprozess jedoch verfrüht ein. Ein weiteres Indiz sind Bienennester, die in Zyklon-Jahren bodennäher als gewöhnlich angelegt werden. Als kurzfristige Indizien deuten wenige Tage vor einem Zyklon bestimmte Wetterlagen auf ihn hin, Tiefseefische werden gehäuft in Meeresbuchten und Flussläufen gesichtet und Meeresvögel halten sich im Landesinneren auf. All diese Indikatoren sind mit erheblichen Unsicherheiten versehen, in Summe helfen sie jedoch für die Handhabung der Naturgefahren.

Die langfristigen Indikatoren unterstützen bei der landwirtschaftlichen Planung, ob man Risiken eingeht, besonders windanfällige, jedoch ertragreiche Pflanzen wie Kassava vermehrt anzubauen, oder aber zur Ernährungssicherheit wetterresistente, langsamer wachsende Pflanzen wie giant swamp taro zu kultivieren. Kurzfristige Maßnahmen, die negativen Auswirkungen eines nahenden Zyklons zu reduzieren, wären beispielsweise das Zurückschneiden von Kassava-Pflanzen zur Verkleinerung der Wind-Angriffsfläche oder das Beschweren und Festschnüren von Hausdächern.

Im Vorfeld von Zyklon Evan, welchen ich am 17. Dezember 2012 in Dravuni miterlebte, wurden mir auf den Arbeitswegen zu den Feldern viele Anzeichen gezeigt, ohne dass auf diese jedoch reagiert wurde. Ebenso erzähle mir die erfahrenste Fischerin (62) des Dorfes Malawai, dass es bezüglich Zyklon Tomas vom März 2010 genug Anzeichen gegeben hatte, die sie als Kind gelernt hatte, dass dieser Zyklon bevorstünde, ohne dass sie oder andere Dorfbewohner darauf reagiert hätten. Das Wissen, dass der Klimawandel zu einer Verschiebung von Jahreszeiten führen kann, untergräbt die Glaubwürdigkeit von Mango- und Brotfruchtbäumen als Zyklon-Indikatoren. Das traditionelle Wissen findet kaum noch Anwendung, da die Zeichen nunmehr im Zuge des Klimawandels so umgedeutet werden, dass auf Muster und Abfolgen der Vegetation und des Wetters kein Verlass mehr sei. 
Hochtechnisierte Frühwarnsysteme suggerieren eine höhere Verlässlichkeit Zyklone vorherzusagen als traditionelle Indikatoren, sodass man bis zu deren Aufforderung untätig bleibt. Die Erfassung, genaue Verortung und Prognose über Zerstörungspfade gestaltet sich jedoch mitunter schwierig, sodass verlässliche Informationen zu betroffenen Regionen, Windstärken und Fluthöhen teilweise nur wenige Stunden im Voraus geäußert werden. Das große Potential, dass das durchaus noch vorhandene traditionelle Wissen birgt, wird verdrängt und bleibt ungenutzt.

Wird ein Zyklon dann via Radio, Fernsehen, Zeitung, Internet sowie über mobile Textnachrichten angekündigt, folgen zumindest viele den Anweisungen und suchen gemeinsam die geeignetsten Schutzräume auf - zumeist die Kirche oder ein Schulgebäude. Im Falle von Tsunamis hingegen herrscht aufgrund fehlender Selbsterfahrung wenig Sensibilisierung. In Malawai und Nukui haben lediglich die Ältesten Kindheitserinnerungen an eine Flutwelle Anfang der 1950er. Aufgrund pazifischer Identitäten bestand jedoch große Empathie zu den Opfern eines Tsunami, der im September 2009 an der Küste Samoas enorme Schäden versursachte und 143 Menschenleben forderte (Weber 2013: 180). Auf eine TsunamiWarnung am 6. Februar 2013, ausgelöst von einem Erdbeben der Stärke 8,0 vor den Salomonen (NOAA 2013), reagierte mein Umfeld jedoch überhaupt nicht. Aufgrund zu vieler falscher Alarme bezüglich Tsunamis - zu denen, was Fidschi betraf, auch diese Warnung gehörte - werden Tsunami-Warnungen vom Großteil der Küstenbewohner ignoriert. Schlimmer noch werden mitunter die Küsten bewusst aufgesucht, um sich als Schaulustige die Phänomene anzusehen.

Externe Interventionen zur Klimawandelanpassung und zum Katastrophenschutz-Management unterstützen existierende Wissens- und Handlungsformen daher nicht zwangsläufig. Sie können dieses Kulturgut zerstören und die Anpassungsfähigkeit der lokalen Gemeinschaften schädigen (Thaman 1982; Weber 2013: 181). Sie können jedoch auch nicht pauschal als Fehlentwicklungen kritisiert werden. Sofern ihr Vorgehen kulturell sensibel gestaltet ist, können sie sehr wohl zur Sicherheit beitragen. Aufklärungskampagnen über Verhaltensstrategien in Notsituationen können beispielsweise so konzipiert sein, dass sie bestehende soziokulturelle Anliegen unterstützen. Inhaltlich könnten Hilfestellungen unter anderem darin bestehen verwundbare Menschen zu identifizieren - dies können zum Beispiel Kleinkinder, Ältere oder Behinderte sein -, ihnen bei der Erreichung eines sicheren Ortes effektiv zu helfen und vorsorglich die Räumlichkeiten mit Trinkwasser und Verbandsmaterial auszustatten.

Besonders vielversprechend sind praktische Wissensvermittlungen, die nicht einseitig auf das Ziel der Reduzierung von Naturgefahren aus sind, sondern vielseitiger das kulturelle Werteschema unterstützen. So helfen z.B. workshops, bure zu bauen, nicht nur, widerstandsfähige Häuser zu bauen und im Falle einer Hauszerstörung schnell und kostengünstig ein neues zu errichten. Sie können auch im Alltag sinnvoll genutzt werden und stärken die kulturelle Identität. 


\subsubsection{Erfahrungen mit Zyklonen in Malawai und physisch-materielle Reaktionen}

In Malawai initiierte ich im Januar 2010 mehrere Auswirkungsdiagramme tropischer Zyklone (siehe Abb. 16). Die Darstellung beruhte im Wesentlichen auf den Erfahrungen mit den zwei für die Dorfgemeinschaft bis dato zerstörerischsten Zyklonen der jüngeren Vergangenheit, Kina 1992/93 und Bebe 1972.

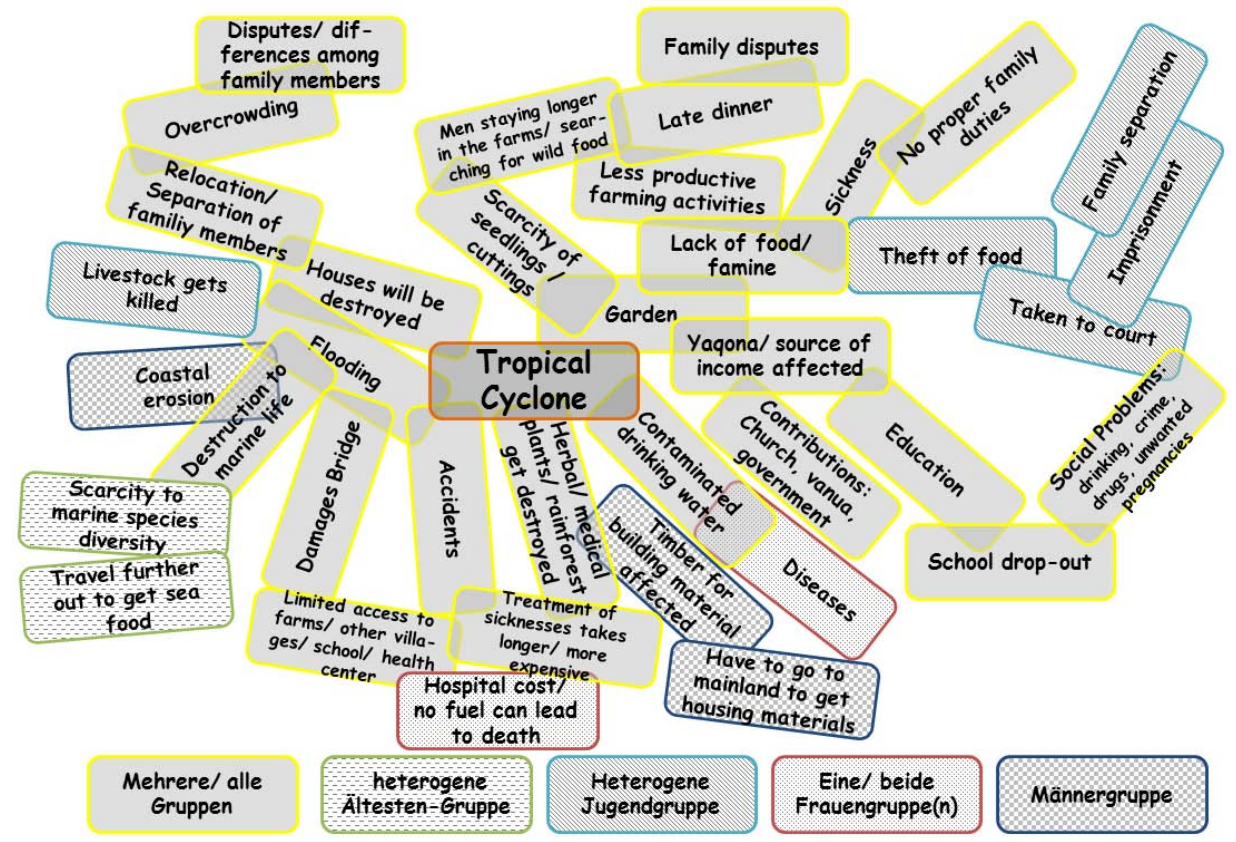

Abb. 16: Auswirkungsdiagramme tropischer Zyklone

Beide Zyklone verwüsteten jeweils den Großteil die Felder. Bei solchen Extremereignissen werden windanfällige Pflanzen wie Kassava entwurzelt. Da yaqona an Hängen angebaut wird, sind insbesondere Jungpflanzen anfällig für Sturmschäden. Stehengebliebene Pflanzen wiederum drohen aufgrund der Bodenfeuchte zu verfaulen. Daher wird ein Großteil der Ackerpflanzen abgeerntet. Entsprechend der Möglichkeiten, diese haltbar zu machen, ist man im ersten Monat nach dem Zyklon noch gut versorgt. Bestimmte Kassava- und Süßkartoffel-Sorten sind bereits nach drei Monaten erntereif und ganzjährig anpflanzbar. Der Staat liefert gemeinsam mit internationalen Hilfsorganisationen Hilfsgüter in die betroffenen Gebiete. Aufgrund der stürmischen See, einer begrenzten Anzahl an Schiffen, und einer geringen Priorität Gau Islands wegen ihrer kleinen Einwohnerzahl von unter 3.000 Bewohnern, treffen erste Hilfsgüter erst Wochen nach dem Zyklon ein. Sie füllen damit die mögliche Ernährungslücke, sodass auch im Zuge eines Zyklons kein Hunger herrscht. Weiterhin besteht traditionelles Wissen um wildwachsenden Jams und anderen Nahrungsquellen aus dem Regenwald, die den Speiseplan er- 
gänzen. Die aufgewühlte See behindert zudem die gängigen Fischfangmethoden. Korallen leiden direkt unter der Wellenenergie sowie noch Wochen und Monate später unter dem erhöhten Einfluss von Frischwasser und Sedimentation aus dem Fluss. Seegrasplantagen überlebten dies nicht. Ernährungsumstellungen können für Einzelne gesundheitliche Beeinträchtigungen nach sich ziehen. Wenn die Zyklone stürmen, flüchten die Bewohner in die höher gelegene Kirche, dem stabilsten Gebäude Malawais. Speziell ältere Menschen mit körperlichen Beschwerden haben dabei Schwierigkeiten, den Anstieg unter den erschwerten Wetterverhältnissen zu bewältigen, ihnen wird jedoch von den anderen im Dorf geholfen. Schwerwiegende Verletzungen oder gar Todesfälle hat es bisher nie gegeben.

Im Zuge der Naturgewalten, die bei Bebe 1972 wirkten, weitete sich der Fluss Malawai und eine ganze Häuserreihe wurde unwiderruflich weggespült. Vorher konnte der Stamm einer einzelnen Kokosnusspalme als Brücke dienen, seitdem die Flussufer erodierten, ist eine aufwändigere Brückenkonstruktion notwendig. Die Fläche zwischen Fußweg und Küste, auf der unter anderem die heutige Gemeindehalle steht (siehe Karte 3), wurde im Zuge des Zyklons ebenfalls vom Meer vereinnahmt. Die Konstruktion des Deiches ist höchstwahrscheinlich eine direkte Reaktion auf Bebe, mit dessen Hilfe das Gebiet wieder reklamiert werden konnte. Die fortgespülten Häuser wurden in höheren Lagen neu errichtet. Aufgrund von kontaminiertem Trinkwasser im Zuge von Erosionsprozessen verschärfte sich die gesundheitliche Belastung der Haushalte, seit der neuen Trinkwasseranlage 1985 ist diese Gefahr jedoch weitestgehend eingedämmt.

Kina zerstörte vier Häuser vollständig. Die Besitzer bekamen Monate später je $1.000 \mathrm{FJ}-\$$ Kompensation vom Staat. Das Geld musste jedoch persönlich in Suva abgeholt werden, was Vorkosten und mehrmonatige Verzögerungen bedeutete. Der Wunsch weiter Hang aufwärts zu bauen, verstärkte sich. Zudem wurden als Reaktion auf Kina erstmals Wohnhäuser mit Wänden aus Zement errichtet.

Im Falle des Zyklon Tomas vom März 2010 wurde eine Warnung fünf Tage vorher per Radio übermittelt, dass sich nördlich Fidschis ein Zyklon oder aber zumindest eine Depression bilde. Am nächsten Tag wurde Entwarnung ausgerufen. Etwa anderthalb Tage, bevor er für Gau Island seinen Höhepunkt erreichte, meldete man ihn als Zyklon der Stufe vier mit Kurs auf den Nordosten Fidschis. Zu diesem Zeitpunkt waren die Dörfer im Nordosten Vanua Levus und benachbarter Inseln bereits voll vom Zyklon erfasst. Auch viele Bewohner Malawais unterschätzten die Gefahr. Während manche vorsichtshalber bei Verwandten in den sichereren Häusern Hang aufwärts übernachteten, tranken andere zum Teil noch bis spät abends yaqona und wurden dann im Schlaf in ihren Häusern überrascht, als ab zwei Uhr Nachts die untere Dorfhälfte überschwemmte.

Zwei interviewte ${ }^{69}$ Dorfälteste (78 und 59 Jahre), welche Bebe und Kina miterlebt hatten, sahen Tomas als den schlimmsten an. Aufgrund seit einer Woche

${ }^{69}$ Am Morgen des 16. März 2010 erreichte Tomas seinen Höhepunkt für Gau Island. Meine Forschungsassistenten Maleli Qera und Eri Balenacagi führten die Interviews im Rahmen einer dreitägi- 
anhaltender starker Niederschläge hatte der Fluss Hochwasser und der Wind trieb das Meerwasser ins Dorf. Zwei Drittel der Häuser wurden überschwemmt, die exponiertesten Häuser standen knapp zwei Meter unter Wasser. Obwohl die partiell gepflanzten Mangroven einen Teil der Wellenenergie absorbierten, wurde der Deich fast vollständig zerstört, das Wellblech-Wohnhaus auf der anderen Flussseite wurde zusammen mit der Brücke fortgespült, eine Zementwand der Gemeindehalle wurde eingerissen, der Shop und drei weitere Wohnhäuser aus Zement nahe des Flusses wurden zerstört. Während die überraschten Dorfbewohner mitten in der Nacht mit ihren wichtigsten Habseligkeiten in die Kirche flüchteten, wobei die jungen Männer den Frauen, Alten und Kindern halfen, hob das Wellblechdach eines Hauses ab. Insbesondere die Lautstärke dieses Vorgangs rief zum Teil Panik hervor (Vorsitzender der Jugendgruppe, 28; Farmer, 51).
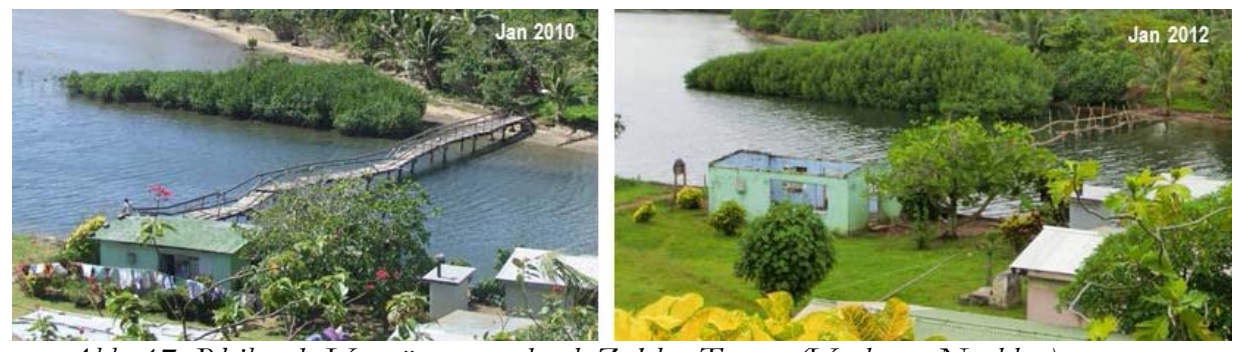

Abb. 17: Bleibende Verwüstungen durch Zyklon Tomas (Vorber - Nachber)

Als Brücke dient auch drei Jahre nach dem Zyklon eine Behelfsbrücke aus Kokosnussstämmen (siehe Abb. 17). Ihre Überquerung ist ein Balanceakt, vor dem insbesondere die jüngeren Grundschulkinder, die den Fluss für ihren Schulweg überqueren müssen, anfänglich Angst hatten. Zwei der zerstörten Häuser wurden wieder aufgebaut, eines wurde aufgegeben. Das vierte wurde weiter hangaufwärts neu errichtet da man nun mit häufiger wiederkehrenden Überschwemmungen des Flusstals rechnet. Da das Haus ursprünglich vom Großvater des derzeitigen Ramasi gebaut wurde, fiel die Aufgabe des alten Standortes besonders schwer (Ramasi, 78). Dies veranschaulicht, wie deutlich die Härte des Schocks wahrgenommen wurde. Um dem Risiko zu entgehen, geben die Bewohner durch den Trend, Hang aufwärts zu ziehen, sukzessive ihr vanua auf. Die Einsicht, Identifikationsmerkmale aufgeben zu müssen, zeugt aufgrund der Bedeutung, die der Ahnenglaube für die Menschen einnimmt, von einem ausgesprochen hohen Risikobewusstsein sowie entsprechend großen Adaptionsmöglichkeiten.

gen Bestandsaufnahme durch, die aufgrund witterungsbedingter Transportprobleme dreimal verschoben werden musste und erst Ende Mai 2010 stattfand. Mein Hauptforschungsaufenthalt endete wenige Wochen vor dem Ereignis. Im Januar 2012 und Januar 2013 war ich nochmal zu Besuch. 


\subsubsection{Soziale Sicherung als Anpassung an Naturgefahren}

Der Bau und die Instandhaltung des Deiches, die Substitution der Bausubstanz Holz durch Zement und die beginnende Verlagerung der Wohnhäuser Hang aufwärts, sind Bewältigungsstrategien, sich den schädlichen Auswirkungen der Naturgefahren zu entziehen. Besonders schweren Zyklonen halten diese baulichen Verbesserungen jedoch nicht Stand. Die wichtigsten Handlungen zur Sicherung gegenüber Naturgefahren sind nicht physisch-materieller, sondern soziokultureller Natur.

Die Verwüstung von Feldern und Zerstörung von Häusern trifft die einzelnen Haushalte eines Dorfes unterschiedlich stark. Dank des intakten sozialen Zusammenhaltes und bestehender kultureller Praktiken des sozialen Ausgleichs wie kerekere, finden Menschen ohne Obdach und ohne Mittel Zuflucht in der Gemeinschaft. Die Familien, deren Häuser zerstört werden, finden Unterschlupf in der Kirche sowie bei Verwandten. Das Zusammenleben mehrerer Familien unter einem Dach und das gemeinsame Zubereiten von Mahlzeiten stellen zentrale Bewältigungsmechanismen dar. Aufgrund der angespannten Situation, der Überbelegung und damit verbundener mangelnder Privatsphäre stellt die Maßnahme ihre eigene Basis, das soziale Miteinander, auf die Probe. So kann ein Zyklon zu innerfamiliären Streitigkeiten führen. Die eigene Lebensqualität hängt jedoch entscheidend von der Qualität des Zwischenmenschlichen ab. In der Wahrnehmung der Betroffenen stellen Belastungen des Sozialgefüges die größten Bedrohungen eines Zyklons dar, wie bei den Auswirkungsdiagrammen tropischer Zyklone auf verschiedene Arten herausgearbeitet wurde (siehe Abb. 17).

Dank der gegenseitigen Hilfe werden die in Mitleidenschaft geratenen Häuser nacheinander seitens der gesamten Dorfgemeinschaft wieder aufgebaut. So entspannt sich die Wohnsituation sukzessive und binnen eines Monats sind fast alle Häuser wieder bezugsfähig. Würden sich die Familien einzeln ausschließlich um ihre eigenen Schäden kümmern, würde sich der Prozess wesentlich in die Länge ziehen. Aufgrund der Verwüstungen der Felder müssen manche zeitnah abgeerntet werden. Andere Felder hingegen können länger gehalten werden. Auch wenn die Felder Einzelpersonen zugeordnet sind, stehen die Familien beim Zyklon zusammen und bewirten sich gegenseitig um effizient Überfluss und Mangel an Nahrung auszugleichen.

Menschliche Sicherheit wird entsprechend in erster Linie über die soziokulturelle Einbettung in die Gemeinschaft erzeugt. Das soziale Miteinander, welches den Sicherungsanker in Notsituationen bildet, wird aus kulturellen Motiven bewahrt. Die wichtigsten Strategien die Gefahren eines Zyklon zu minimieren, zielen demnach nicht primär auf den Schutz vor Naturgefahren ab. Die Bedeutung des Gemeinschaftlichen ist in einer Vielzahl an Bräuchen kulturell verankert, die Stärke einer Gemeinschaft wird im Alltag beständig reproduziert und das Gemeinwohl bildet den wichtigsten Grundpfeiler individueller Lebensqualität. Gezielte Strategien zur Linderung von Naturgefahren sind von zweitrangiger Bedeu- 
tung. Das - vom Zyklon völlig unabhängig gedachte - Bemühen um eine intakte soziale Gemeinschaft im gelebten Alltag ist die primäre Quelle einer geringen Verwundbarkeit gegenüber Naturgefahren. Die Auslebung und Bewahrung der Kultur, welche sich in sozialer Sicherung ausdrückt, sind die am besten angepassten Strategien zur Reduzierung von Verwundbarkeit gegenüber Naturgefahren.

Intergenerationell erprobtes, angepasstes Wissen hat auf Fidschi widerstandfähige, starke Solidargemeinschaften entstehen lassen. Diese kulturellen Werte greifen auch bei der Handhabung von Naturgefahren und haben Potentiale, den Klimawandel zu meistern. Neue Wissensformen können nicht zwingend an das implizite Wissen andocken, da diese auf einem gesellschafts-kulturell westlich eingebetteten Hintergrundwissen basieren, welches bei den Fidschianern aber unzureichend ausgeprägt ist. Analysen, es existiere ein mangelndes Bekämpfungspotential aufgrund eines unzureichend ausgeprägten naturwissenschaftlichen Verständnisses oder aber mangelndes Bewusstsein über die vom Klima ausgehenden Gefahren, greifen zu kurz, sofern sie den kulturellen Wertehorizont und die darauf aufgebaute traditionelle Wissensbasis verkennen. Programme, die fidschianische Dorfbevölkerung besser auf den Klimawandel und Naturgefahren vorzubereiten, müssen bei der Wissensaufbereitung und -vermittlung hoch sensibel vorgehen. 


\section{Fazit}

Diese Schlussbetrachtung widmet sich sowohl den zentralen Erkenntnissen, als auch den Limitationen dieser Studie. Inhaltlich wurde zum Themenkomplex Sozialer Sicherung bei gesellschaftlicher Transformation gearbeitet, um Einsichten in Bedingungen für „gutes Leben“ aus emischer Perspektive fidschianischer Küstendorfbewohner zu erlangen. Methodologische Überlegungen partizipativer Forschung und der Ethnographie wurden für die interkulturelle Humangeographie aufbereitet. Ziel war es, mit der gewählten Methodik die kultur- und sozialgeographische Forschung im globalen Süden zu befruchten. Fußend auf Basisbegriffen der Sozial- und Kulturwissenschaften und angereichert um entwicklungspolitische Anregungen und geographische Verwundbarkeitskonzepte wurde ein integratives Konzept zu sozialer Sicherung erstellt. Die gewonnen Erkenntnisse sollten dabei Anknüpfungspunkte, jedoch keine direkten Handlungsempfehlungen für politische Anwendungsbezüge aufweisen. Diesen inhaltlichen, methodischen und praktischen Zielen wollte ich unter möglichst hohen empirisch-wissenschaftlichen wie ethischen Ansprüchen gerecht werden, wobei die Auffassung verfolgt wird, dass Qualität mit ethischen Prinzipien einhergeht. Weiterhin sollte die interkulturelle Humangeographie bereichert werden, indem insbesondere Fallstricke eines Eurozentrismus überwunden werden.

\section{Reflektion des partizipativen, methodischen Vorgehens}

Die Forschung hat sich den Potentialen fidschianischer Küstendorfbewohner gewidmet, ihre Vorstellungen eines guten Lebens zu verwirklichen. Aufgrund der Interkulturalität der Forschungssituation wurde eine möglichst offene Forschungshaltung eingenommen, um einer Projektion eigener bzw. westlicheuropäischer Vorstellungen eines guten Lebens auf fidschianische Gesellschaften vorzubeugen. Als hilfreich haben sich partizipative Forschungsmethoden erwiesen, seien es teilnehmende Beobachtung oder Methodensets mit Fokusgruppen im Sinne der PRA-toolbox. Die Studie zeigt jedoch, dass es seitens des Forschers einer Empathie und eines intrinsischen Interesses an den Menschen bedarf, der weit über das spezifische Forschungsinteresse hinausgeht, um kulturelle Spezifika erfahren, verstehen und gewichten zu können. Dieser Arbeit liegt ein normatives Wissenschaftsverständnis zugrunde; ein hoher Grad an Partizipation ist angestrebt. Forschende möchten demnach möglichst ganzheitlich am gesellschaftlichen Leben teilnehmen, was sozio-kulturelle, politische und ökonomische Tätigkeiten einschließt - und die Mitmenschen am eigenen Leben teilhaben lassen. Obwohl ein solches Einlassen auf die „Untersuchungs“-welt dabei weitgehend unabhängig der eigentlichen Forschungsfrage geschieht, bereichert solch ein soziales Verhalten ungemein das Forschungsergebnis. Diese Arbeit bestätigt, dass ethnographische Forschungsethiken nicht nur in ethnologischen Fachrichtungen, sondern auch in der Humangeographie als geeigneter Schlüssel zur Vereinbarkeit 
von Ethik und Forschungsqualität dienen. Verzerrungen, die mit einer auf die individuelle Persönlichkeit des Forschenden aufbauenden Untersuchung unweigerlich einhergehen, kann mittels kritischer Selbstreflektion und Triangulation weitestgehend entgegengewirkt werden.

Im Vergleich zur geringen Größe und Bedeutung, die Fidschi im globalen Maßstab einnimmt, existieren dazu verhältnismäßig viele geographische und ethnologische Studien, selbst in deutscher Sprache. Soziokulturelle Praktiken, Vorstellungen und Phänomene wie vanua, yaqona-Zeremonien, Ahnenkulte, das tabu oder kerekere wurden bereits wissenschaftlich aufbereitet. Im Zuge des hier gewählten, offenen und partizipativen Zugangs konnten jedoch neueZusammenhänge erkannt und damit zumindest punktuell das Wissen und Verständnis vertieft werden. Diese Arbeit leistet damit einen Beitrag zu einem ganzheitlicheren Verständnis kultureller Phänomene und dahinterliegender Wertvorstellungen. Gerade in Anbetracht der Limitierungen, dass ich nur rudimentäre Sprachkenntnisse des Fidschianischen besitze, ist dieser Erfolg beachtlich. Anhand der partizipativen, forschungsethischen methodologischen Qualität lässt er sich erklären.

Im Sinne der offenen Forschung bedurfte diese Arbeit eines angepassten Forschungsdesigns. Als interdisziplinäre Bereicherung verstanden strebt die Humangeographie und hierbei insbesondere die Geographische Entwicklungsforschung eine sozialtheoretische Vorkonzeption als Rahmung wissenschaftlicher Forschung an. Auch wenn die Anwendung von Denkmodellen international renommierter Sozialtheoretiker Anfang der 2000er ein richtungsweisendes Ideal war (Dörfler et al. 2003; Tröger 2003), das die Qualität und Sichtbarkeit geographischer Entwicklungsforschung außerordentlich steigerte, geht diese Arbeit einen Schritt weiter. Die hiesige theoretisch-konzeptionelle Rahmung soll der Aufforderung Vernes (2012) folgend eine nuancierte Kollage verschiedener soziologischer Strömungen darstellen, die so zusammengestellt ist, dass sie explizit auf den Untersuchungsgegenstand angepasst ist. Zeitlich im Forschungsverlauf betrachtet läuft die konzeptionelle Arbeit daher parallel zur Empirie. Eine völlige Um- bzw. Abkehr sozialtheoretisch fundierter Forschung, nach welcher die Empirie zuerst erhoben würde um diese anschließend mit sozialtheoretischen Konzepten abzugleichen; ein solches Experiment induktiver Forschung hat diese Arbeit nicht angestrebt.

\section{Postkoloniale und transkulturelle Beschaffenheit der fidschianischen Gesellschaft}

Fidschi befindet sich in einer gesellschaftlichen Transformation. Dies beinhaltet einen beschleunigten Wandel gesellschaftlicher Praktiken, aber auch einer Verschiebung oder Spreizung zugrundeliegender gesellschaftlicher Wertmaßstäbe. Die Veränderung ist vielschichtig, dynamisch und raum-zeitlich inkonsistent. Die Komplexität der gesellschaftlichen Transformation konnte die vorliegende Arbeit nicht hinlänglich aufschlüsseln, wohl aber gezielte lokale Einblicke liefern um die Spannweiten und Dimensionen solcher Veränderungen zu skizzieren.

Die fidschianische Gesellschaft stellt eine einzigartige Mischung aus vorkolonialen, kolonialen und postkolonialen Denk- und Lebensweisen dar. Sie ist ein his- 
torisch gewachsenes kulturelles Hybrid aus lokalen, regional-pazifischen, westlichen und globalisierten Elementen. Diese Zusammenstellung verleiht ihr einen eigenständigen Charakter, den sowohl Außenstehende als auch die fidschianische Bevölkerung als eigene Kultur wahrnehmen, die ihren Bewohnern Identität stiftet. Als tragende Säulen von Identität und Wohlbefinden halten sich vanua, matanitu, und lotu, die sich verkürzt mit „Land“, „Herrschaftsgewalt“ und „Religion“ übersetzen ließen. Die Arbeit bestätigt Konzeptionen von Kultur, nach denen dies kein statisches, geschlossenes oder absolut akzeptiertes Set darstellt, sondern dynamischen immerwährenden Aushandlungsprozessen unterliegt. Durchlässigkeiten gesellschaftlicher Praktiken und Wertvorstellungen gegenüber anderen Kulturen untergraben dabei nicht die spezifischen Eigenheiten und Abgrenzungsmöglichkeiten einer Kultur. So wird in Fidschi die Ausgestaltung der Kernelemente vanua, matanitu und lotu kontrovers diskutiert, eine Loslösung von einem der soziokulturellen Grundpfeiler jedoch von nahezu keinem Küstendorfbewohner angestrebt. Im Gegenteil werden die Elemente als so intensiv ineinander verschränkt wahrgenommen, dass allein schon eine separate Betrachtung kaum möglich erscheint.

Vanua ist demnach das Land, auf dem die eigene soziale Gruppe lebt. Je nach Kontext kann diese beispielsweise als Großfamilie, Dorfgemeinschaft, Stamm oder Nation definiert werden, hat entsprechend unterschiedliche räumliche Dimensionen und impliziert stets bestimmte sozio-politische Herrschaftsordnungen. Zugleich ist vanua das Land, in dem die Ahnen leben und durch welches sie wirken. Es ist daher nicht unabhängig einer spirituellen Dimension denkbar und bedient in erster Linie keine physisch-materiellen, sondern kognitive Raumvorstellungen. Im Zuge der Kolonialisierung wurde ein Landrechtssystem geschaffen, mit welchem darüber hinaus marktwirtschaftliche Wertvorstellungen Einzug in das fidschianische Verständnis von vanua erhalten haben. Je nach Kontext ergänzen sich die Bedeutungssphären oder aber sie konfligieren.

Matanitu kann normativ als göttliche Ordnung des sozialen Raumes erfasst werden, sodass auch hier die drei Säulen zusammenfallen. Dies lässt jedoch erheblichen Interpretationsspielraum zu, in wie fern real existierende Herrschaftssysteme dem göttlichen Ideal entsprechen. Allgemein stoßen die unteren Gliederungsebenen des traditionellen Häuptlingssystems auf große Akzeptanz. Hierüber wird jedem Gemeindemitglied eine soziale Rolle und Aufgabe zugeschrieben, die einem Identität und Status verleiht. Sie bietet einen schützenden Rahmen, innerhalb dessen man sich selbst verwirklichen kann - und lässt begrenzt Möglichkeiten zu, sich alternative soziale Funktionen zu suchen. Der Große Häuptlingsrat als nationale und seitens der ersten Kolonialregierung erschaffene Instanz wird wesentlich kritischer begutachtet. Das nationalstaatliche, ans britische Demokratieverständnis angelehnte Regierungssystem wird als künstlich betrachtet und sorgt für erhebliche Reibungen in der Gestaltung des Alltags.

Lotu bezieht sich auf die spirituelle Dimension, aus der sich die Normen und Werte des Zusammenlebens ergeben. Sie stiftet menschlichem Leben ihren Sinn 
und zeigt sich weltlich-materiell in der Qualität des (Zusammen-)Lebens. Die christliche Kirche ist nicht als identisch mit lotu zu betrachten. Auch wenn die christliche Lehre die mit Abstand bedeutendste Quelle der Inspiration für gutes Leben darstellt, wird ihre Exegese sowie der weltliche Aufbau der Kirche kontrovers diskutiert.

\section{Kultur des Umgangs mit Konflikten im Zuge der Transformation}

Die grundlegende Argumentation dieser Studie besagt, dass Ideale für gutes Leben wie Lebensqualität oder Gerechtigkeit nicht final definiert werden können. Die zugrunde liegenden Vorstellungen und Konzepte sind historisch gewachsen, räumlich differenziert und verändern sich. Die Kriterien, nach denen gewertet wird, werden kontrovers diskutiert. Aus der Diskussion über konfligierende Praktiken und Werte mit den und unter den Dorfbewohnern ergibt sich die Erkenntnis, dass öffentlicher Vernunftgebrauch praktiziert wird. Auf Basis des theoretisch-konzeptionellen Hintergrundes bildet die Tatsache, dass individuelle Bedenken und gesellschaftliche Missstände öffentlich thematisiert werden können, den entscheidenden Faktor, Lebensqualität bewahren und steigern zu können. Öffentlicher Vernunftgebrauch hat hierbei eine spezifisch kulturelle Dimension. Die soziale Praxis der Streitlösung ist kulturell eingebettet. Mit anderen Worten herrscht auf Fidschi eine Konfliktkultur. Grenzen des öffentlichen Vernunftgebrauchs bestehen vor allem darin, wenn Menschen keinen Bezug zu dieser Diskussionskultur aufweisen oder aber diese ablehnen, worunter Auswärtige oder ins gesellschaftliche Abseits Geratene fallen können. Weiterhin limitiert die Intransparenz diktatorischer Regierungsapparate, welche freie Medien beschneiden, die öffentliche Meinungsäußerung und Meinungsbildung gerade in politischer Dimension.

Besonders im Rahmen von yaqona-Zeremonien wurde deutlich, wie Streitthemen respektabel diskutiert werden können. Der Respekt gegenüber den Ahnen, welcher sich zugleich im Respekt gegenüber matanitu, vanua und lotu und sich damit gegenüber der Diskussionspartner niederschlägt, schafft einen kulturellen Rahmen zur Konfliktlösung. Die Transformation und kulturelle Werte stehen in Wechselwirkung zueinander. Die größte potentielle Gefährdung der Lebensqualität wäre demnach ein möglicher Wegfall dieses sozio-kulturellen wie spirituellen Fundaments im Zuge der gesellschaftlichen Transformation. Im Rahmen dieser Studie dreier Küstendörfer kann diese Gefahr jedoch als gering eingeschätzt werden. Tradition ist ein Prozess und kulturelle Werte ändern sich über die Zeit. Die Transformation beschleunigt diesen Fortlauf nicht in dem Maße, als dass die eigene kulturelle Identität als Quelle von Sicherheit und Vertrauen bzw. Wohlbefinden verloren ginge. Die Kultur und ihre Narrative weisen eine erstaunliche Persistenz auf. Dieses Fortbestehen liegt in ihrer Anpassungsfähigkeit begründet. Die ambivalente Mischung aus einem klaren, festen, kulturell eingebettetem Wertefundament auf der einen Seite und einer Offenheit Neuem gegenüber auf der anderen 
Seite ermöglicht es, dass positive Auswirkungen der Transformation auf die Lebensqualität überwiegen.

Handlungsstrategisch ergeben sich zwei Extrema den Veränderungsprozessen zu begegnen. Man kann sämtliche Veränderungen unreflektiert gutheißen, was zu einer Aufgabe des Wertesystems führen würde. Beispiele mit entsprechend negativer Auswirkung auf die Lebensqualität sind Drogenmissbrauch oder naive bzw. verklärte Vorstellungen von städtischem Leben und Lohnarbeit, der manche unterliegen. Das andere Extrema ist das Verschließen gegenüber Veränderungen in der falschen Hoffnung kulturellen Stillstand schaffen zu können. Dies kann maximal ein bestehendes Niveau an Lebensqualität bewahren, jedoch keine Steigerungen bewirken. Da die Kultur jedoch mit sämtlichen Sphären des Gesellschaftlichen interagiert, welche sich in stetigem Fortlauf befinden, ist die Bewahrung einer Kultur als Zustand zum Scheitern verurteilt.

Der Mittelweg - auf Basis bestehender Werte zu versuchen, die Transformation in gewünschte Richtungen zu lenken - stellt eine vielversprechende, wenn auch keine einfache Strategie dar. In der Praxis drückt sich die Stärkung kultureller Werte in einem Neotraditionalismus aus. In der Übersteigerung von Traditionen und Narrativen wohnen Momente inne, kulturelle Praktiken aktiv und bewusst als Gegenbewegung zum Wandel zu inszenieren. Zudem wird sich zur Verwirklichung oftmals moderner Elemente in der Umsetzung bedient. Sollten sich die bestehenden Werte dabei verändern, so ist dies im Sinne der Theorie der Strukturierung ein natürlicher Prozess und hinsichtlich sozialer Sicherung ein Zeichen für Anpassung. Diese Begleitphänomene sorgen für Veränderungen des Brauchs und erzeugen mitunter nicht-intendierte Wirkungen.

\section{Neo-traditionelle Strategien zur Erzeugung von Lebensqualität in globalisierten Kontexten}

Die zentrale Strategie, der Transformation zu begegnen, um Lebensqualität zu erzeugen, ist der Versuch einer Lenkung der verändernden Trends in gewünschte Richtungen. Die Komplexität des Wandels ist allerdings nur eingeschränkt begreifbar. Die diffuse Beschaffenheit des Transformationskomplexes behindert daher dessen Steuerbarkeit und hinterlässt Gefühle der Unsicherheit. So können nicht-intendierte, negative Folgen entstehen. In einem stark heruntergebrochenen Beispiel genießt institutionalisierte Bildung einen hohen Stellenwert. Generatoren wurden demnach angeschafft, damit Kinder abends Licht zum Lernen haben. Stattdessen schauen sie jedoch fern.

Demnach können im Neotraditionalismus schädigende Wirkungen stecken. Verlässt man lokale Perspektiven, so geschieht die Schaffung der eigenen kulturellen Identität in Abgrenzung zu möglichen Anderen. Konflikte werden nicht nur geschürt sondern zugleich auch Diskussionsplattformen zur Konfliktlösung torpediert. Wenn Indo-Fidschianern der Zugang zu Land verwehrt wird, trifft dies vor allem Landlose und Kleinbauern - Menschen die schuldlos an möglichen gefühlten Unrechtssituationen der iTaukei sind. Doch der Neotraditionalismus kann auch den iTaukei selbst schädigen, wenn nämlich Potentiale der Selbstver- 
wirklichung durch ethnisch aufgeladene Engstirnigkeit ungenutzt bleiben. Mischehen beispielsweise bleiben bis heute Ausnahmen. Die Forcierung des Traditionellen beschränkt die Möglichkeiten transkultureller Befruchtungen. Im praktischen (Nicht-)Zusammenleben mit den Indo-Fidschianern bleiben enorme Potentiale ungenutzt.

Andere Fallbeispiele zeigen jedoch, wie kritisch reflektiert und durchdacht viele neo-traditionell begründete Handlungsmuster sind und Dank öffentlichem Vernunftgebrauch nicht zu verkürzten Fehlwirkungen führen. So stellt der praktizierte interkulturelle Austausch eine enorme Bereicherung für die fidschianischen Küstendorfbewohner dar. Die internationale Vernetzung fidschianischer Familien und die damit einhergehenden ökonomischen Handlungsspielräume tragen entscheidend zur sozialen Sicherung bei. Das Wissen, welches auswärtige Familienangehörige mittels moderner Kommunikationsmittel in ihre Dörfer tragen, schärft die eigene Identität als Quelle von Lebensqualität in der kritischen Auseinandersetzung mit und Ablehnung von Lebensstilen, die nicht mit den eigenen Werten konform gehen. Zudem schafft die Globalisierung Möglichkeiten, gezielt und informiert Änderungen im gesellschaftlichen Zusammenleben herbeizuführen, die die Lebensqualität der Gruppe steigern.

Diese Regionalstudie zeigt auf, dass Großfamilien wichtige soziale Einheiten bilden. Innerhalb derer findet materieller und immaterieller Austausch statt, welcher auf sozialer Basis elementar zu würdiger Lebensgestaltung und Sicherung beiträgt. Die Familienangehörigen leben jedoch nicht an einem Ort zusammen, sondern bilden globalisierte Netzwerke. Globalisierungschancen bieten nicht nur lukrative Bildungs- und Arbeitsmöglichkeiten im In- und Ausland, dank modernster Kommunikationstechnologien bleiben die Mitglieder unabhängig der räumlichen Distanzen in Kontakt und damit in sozialer Beziehung zu einander.

Die Globalisierung führt im Beispiel jedoch entgegen der Ableitungen in gängigen Diskursen nicht zu einer Loslösung sozialer Gemeinschaften von physischmateriellen Raumbezügen. Für diese Erkenntnis bedurfte es der partizipativen, ethnographischen Erforschung der gesellschaftlichen Raumproduktion. Die spezifischen sozio-kulturellen und spirituellen Raumvorstellungen von vanua und das bestehende Landrecht halten einen Raumbezug aufrecht, sodass die Netzwerke in den Dorfgemeinschaften lokal verankerte Kerne haben. Die Motivation, globale Netzwerke zu spannen, liegt im Wunsch einer Stärkung der Großfamilie und der Dorfgemeinschaft als zentrale Grundpfeiler des eigenen Selbstverständnisses. Mittels der bewussten Lokalisierung kultureller Identitäten betreiben die Gemeinschaften soziale Sicherung und generieren Verwirklichungschancen für ihre Individuen. Als geographische Quintessenz liefert diese Studie eine empirische Bestätigung von Arturo Escobars Vermutung, dass Gesellschaften sich im Zuge der Globalisierung ortsbasiert, wenn auch nicht ortsgebunden, (re-)konstituieren (2011: 138). Kulturelle Unterschiede egalisieren sich nicht; Räume werden soziokulturell spezifisch (re-)produziert. 


\section{Grenzen der Flexibilität neo-traditioneller Entwicklungsstrategien}

Viele Restriktionen des yaqona-Brauchtums sind bewusst aufgegeben worden. Er ist für Frauen und Jugendliche zugänglich gemacht worden, die ihm inzwischen nahezu unreflektiert beiwohnen. Sie sind ins System integriert, was positive Rückkopplungseffekte hinsichtlich der Qualität des öffentlichen Vernunftgebrauchs bewirkt. Die Veralltäglichung und seine Offenheit für Fundraising zeugen von einer Intensivierung, Flexibilisierung und Anpassungsfähigkeit. Auch Menschen, die aus religiösen oder gesundheitlichen Gründen auf den yaqona-Konsum verzichten, nehmen trotzdem an den Sitzrunden teil und bleiben über gegenseitige Respektsbekundungen in den Sitz- und Sprechordnungen gemeinschaftlich integriert.

Sofern man den yavirau als neo-traditionelle Praxis interpretieren will, sind die intendierten wie nicht-intendierten Wirkungen ebenfalls überwiegend positiv. Das Kulturgut wurde nicht nur bewahrt, sondern so attraktiv aufbereitet, dass die Fischtreibjagd Menschen aus Nachbar-dörfern und Verwandte aus Städten anlockt und soziale Bande sowohl lokal als auch überregional stärkt. Lediglich die ökologischen Auswirkungen der Treibjagd auf das Korallenriff können zu bedenken geben.

Die Schaffung eines tabu stellt ein hochgradig erfolgreiches Zusammenspiel aus wissenschaftlich-technischen Erkenntnissen um nachhaltige Fischereiwirtschaft und sozio-kulturellen Wertvorstellungen dar. Die spirituelle Untermauerung einer maritimen Schutzzone als heilige Stätte zur Ehrung der Ahnen sichert die Dorfbevölkerung nicht nur ökonomisch-materiell, sondern stärkt soziale Bande und kulturelle Identitäten. Die Konzeption als Zusammenschluss zweier Dorfgemeinschaften steigert den Wirkungsgrad beachtlich. Der Erfolg der Anlage, welcher Jahre der Selbstrestriktion bedarf, nicht in der Schutzzone zu wildern, lässt sich über die spirituelle und sozio-kulturelle Einbettung dieses Gemeinguts erklären. Nicht jede dieser vielen positiven Wirkungen mag zu Beginn bei der Installation des tabu intendiert gewesen sein.

Als zentrale Erkenntnis sind die fidschianischen Küstendorfgemeinschaften nicht passiv der Globalisierung ausgeliefert, sondern gestalten aktiv ihren Transformationsprozess mit. Trotz existierender Gefahren einer Reduktion individueller menschlicher Sicherheit im Zuge des sozio-kulturellen Wandels entwickeln die Menschen vor Ort Handlungsstrategien, gesellschaftliche Veränderungen ins Sozialgefüge einzubetten um ein gutes Leben entsprechend ihrer sozio-kulturellen und spirituellen Wertvorstellungen auszugestalten. Wenn ein festes Wertefundament gewahrt bleibt, stellt die gesellschaftliche Transformation eine Bereicherung für fidschianische Küstendorfgemeinschaften dar, da sie Handlungsräume zu sozialer Sicherung weitet. Anpassung ist demnach nicht mit Flexibilität und Beliebigkeit gleichzusetzen, sondern baut auf bestehende sozio-kulturelle Werte und diese bedienende Strukturen auf. 


\section{Erkenntnisse für eine (entwicklungs-)praktische Anwendung}

Im Zuge dieser Arbeit konnten die dichotomen Vorstellungen von Mensch und Natur sowie die Künstlichkeit der Unterteilung des Gesellschaftlichen in sozial, wirtschaftlich, politisch und ökologisch als eurozentristisches Gedankengut ausgemacht werden. Dies ermöglichte ein emisch stringenteres, ganzheitlicheres Verständnis des fidschianischen Kultur-Kosmos. Dabei konnten Nachhaltigkeitsvorstellungen im Sinne einer Wahrung der Schöpfung als normatives Gut identifiziert werden. Sie bestehen jedoch im Gegensatz westlicher Handhabungen unabhängig marktwirtschaftlicher Konzepte. Beim Versuch im Rahmen von EZ Nachhaltigkeit zu fördern, können westliche Begründungsmuster entsprechend Dissonanzen hervorrufen. Noch deutlicher wird dies bezüglich Fragen, was „Entwicklung“ sei, da dies kulturell bedingt höchst unterschiedliche Praktiken und Vorstellungen beinhaltet. Daher ist einmal mehr festzuhalten, dass die geographische Entwicklungsforschung einen kritischen Umgang mit dem Entwicklungsbegriff pflegt und weiterhin pflegen muss (Korf \& Rothfuss 2016: 164-166). Gerade im Hinblick auf die Dekonstruktion des Eurozentrismus, eines darauf aufbauenden interkulturellen Forschungsdesigns und einer generellen Öffnung der Wissenschaftswelt für nicht-westliche Forscher und ihrer Erkenntnisse setzt diese Arbeit nur punktuelle Akzente und kann lediglich Potentiale einer solchen Öffnung erkennen lassen. Hier besteht noch großer Handlungsbedarf.

Gerade die Darstellung des Klimawandels und seiner Gefahren erfolgt oftmals sehr verkürzt und ist nicht auf lokale Werte zurechtgeschnitten. Kulturelle Anpassungspotentiale werden verkannt und bleiben ungenutzt, sodass es lokal zu Wahrnehmungsverzerrungen und Fehlentwicklungen kommt.

Im Sinne einer Vorbeugung des Eurozentrismus möchte ich mir keine individuellen Problemlösungskompetenzen für die Menschen vor Ort zuschreiben bzw. anmaßen. Stattdessen kann ich die Eigenständigkeit, Kreativität und den Einfallsreichtum der lokalen Bevölkerung honorieren, mit der sie ihren Ängsten und Sorgen begegnet. Aus dem sozialen Miteinander gewinnen die Menschen die Stärke, ihr Leben sinnhaft zu gestalten. Aufgrund der sozialen Werte stehen individuelles und gemeinschaftliches Wohlbefinden reziprok zueinander. Sie bedingen sich gegenseitig und sind positiv aneinander gekoppelt. Die gegenseitige Achtung fußt auf Toleranz, Respekt und Vertrauen. Mittels einer kulturellen Rahmung, die neben sozialen, wirtschaftlichen und politischen Sphären explizit spirituelle Dimensionen beinhaltet, gelingt es den Menschen, ihren Wünschen, Werten und Idealen auf hohem Niveau gerecht zu werden. EZ kann keinen Nutzen erzeugen, sofern sie frei von lokalspezifischen, kulturellen Kontexten agiert. Möchte man von auBen unterstützend einwirken, plädiere ich dafür bestehende Qualitäten zu unterstützen, die Bedeutung des Kulturellen bei einer Zusammenarbeit anzuerkennen und wertzuschätzen und entsprechend sensibel vorzugehen. 


\section{Literaturverzeichnis}

Abramson, Allen (2004): A small matter of rent to be paid. Towards an analysis of neo-traditional direct action in contemporary Fiji. In: van Meil, Toon \& Jelle Miedema (Hg.): Shifting images of identity in the Pacific, Leiden, S. 75-99.

Acosta, Alberto (2009): Das „Buen Vivir”. Die Schaffung einer Utopie. In: Juridikum 2009, 4, S. 219-23.

Adger, W. Neil (2006): Vulnerability. In: Global Environmental Change 16, S. 268281.

Adger, W. Neil (2010): Social Capital, Collective Action, and Adaptation to Climate Change. In: VOSS, Martin (Hg.): Der Klimawandel. Sozialwissenschaftliche Perspektiven, Wiesbaden, S. 327-345.

Adger, W. Neil, Barnett, Jon, Brown, Katrina, Marshall, Nadine \& Karen O’Brien (2013): Cultural dimensions of climate change impacts and adaptation. In: Nature Climae Change, 3, 2, S. 112-117.

Adger, W. Neil, Brooke, Nick, Bentham, Graham, Agnew, Maureen \& Siri Eriksen (2004): New indicators of vulnerability and adaptive capacity. Tyndall Centre for Climate Change Research, Technical Report 7.

Adger, W. Neil, Dessai, Suraje, Goulden, Marisa, Hulme, Mike, Lorenzoni, Irene, Nelson, Donald R., Naess, Lars O., Wolf, Johanna \& Anita Wreford (2009a): Are there social limits to adaptation to climate change? In: Climatic Change, 93, S. 335-354.

Adger, W. Neil, Lorenzoni, Irene \& Karen L. O’Brien (2009b): Adaptation now. In: Adger, W. Neil, Lorenzoni, Irene \& Karen L. O’Brien, (Hg.): Adapting to Climate Change: Tresholds, Values, Governance, Cambridge, S. 1-22.

Adloff, Frank \& Steffen Mau (2005): Zur Theorie der Gabe und Reziprozität. In: Dies. (Hg.): Vom Geben und Nehmen. Zur Soziologie der Reziprozität, Frankfurt am Main, S. 9-57.

Agrawala, Shardul, Ota, Tomoka, Risbey, James, Hagenstad, Marca, Smith, Joel, van Aalst, Marten, Koshy, Kanyathu \& Biman Prasan (2003): Development and Climate Change in Fiji: Focus on Coastal Mangroves. Organisation for Economic Cooperation and Development (OECD).

AIACC (Assessments of Impacts and Adaptation to Climate Change) (2007): Modeling Climate Change Impacts on Viti Levu (Fiji) and Aitutaki (Cook Islands). Project No. SIS09.

Aigner, Petra (2013): Integration, Interkultur oder Diversität? Anmerkungen zu Fragen von Theorie und Praxis ethnisch - kultureller Vielfalt in Österreich. In: WISO 36, 2, S. 15-41. 
Alongi, Daniel M. (2008): Mangrove forests: Resilience, protection from tsunamis and responses to global climate change. In: Estuarine, Coastal and Shelf Science 78, S. 1-13.

Anthes, Richard A., Corell, Robert W., Holland, Greg, Hurrell, James W., MacCracken, Michael C. \& Trenberth, Kevin E. (2006): Hurricanes and Global Warming - Potential Linkages and Consequences. In: Bulletin of the American Meterological Society 87, S. 623-628.

Arendt, Hannah (1955): Elemente und Ursprünge totaler Herrschaft, Frankfurt am Main.

Arendt, Hannah (1959/2000²): Freiheit und Politik. In: Ursula Ludz (Hg.): Hannah Arendt. Zwischen Vergangenheit und Zukunft. Übungen im politischen Denken I, München, S. 201-226.

Arendt, Hannah (1970): Macht und Gewalt, München.

Arendt, Hannah (1993): Was ist Politik? Fragmente aus dem Nachlaß, München.

Aristoteles (ca. 335-323 v. Chr./200412]): Der Staat als natürliche Voraussetzung glücklichen Lebens, in: Hörster, Norbert (Hg.): Klassische Texte der Staatsphilosophie, München, S. 48-59.

Arsel, Murat (2012): Between 'Marx and markets'? The state, the 'left turn' and nature in Ecuador. In: Tijdschrift voor Economische en Sociale Geografie, 103, 2, S. 150-163.

Bainimarama, Josaia Voreqe (2007): Statement by H.E. Commodore Josaia Voreqe Bainimarama, Prime Minister of the Republic of Fiji Islands, 62nd Session of the UN General Assembly, 28. September 2007, New York.

Baldacchino, Godfrey (2009): Thucydides or Kissinger? A Critical Review of Smaller State Diplomacy. In: Cooper, Andrew F. \& Timothy M. Shaw (Hg.): The Diplomacies of Small States. Between Vulnerability and Resilience, London, S. 21-40.

Barnett, Jon (2010): Human rights and vulnerability to climate change. In: Humphreys, Stephen (Hg.): Human rights and climate change, Cambridge, S. 257-271.

Barnett, Jon \& W. Neil Adger (2007): Climate change, human security and violent conflict. In: Political Geography 26, S. 639-655.

Baumann, Pari \& Subir Sinha (2001): Linking Development with Democratic Processes in India: Political Capital and Sustainable Livelihoods Analysis. Overseas Development Institute, Natural Resource perspectives 68.

Bayliss-Smith, Tim (1976): Koro in the 70's: Prosperity through Diversity? UNESCO/UNFPA Project on Population and Environment in the Eastern Islands of Fiji, Project Working Paper No. 7. Canberra.

Beck, Ulrich (1997): Was ist Globalisierung? Irrtümer des Globalismus - Antworten auf Globalisierung, Frankfurt am Main. 
Beck, Ulrich (2007): Weltrisikogesellschaft. Auf der Suche nach der verlorenen Sicherheit. Lizenzausgabe für die Bundeszentrale für politische Bildung, Bonn.

Bedford, Richard (1985): Population movement in a small island periphery: The case of eastern Fiji. In: Chapman, Murray R. \& Mansell Prothero (Hg.): Circulation in Population Movement: Substance and Concepts from the Melanesian Case, London, S. 333-359.

Benda Beckmann, Franz von (2005): Übersetzung, Vergleich, Transformation: Das lästige Recht der Anderen. Antrittsvorlesung an der Martin-Luther-Universität vom 07. Juli 2005, Halle-Wittenberg.

Bendt, Heinz (2006): Militärputsch in Fidschi. In: Kurzberichte aus der internationalen Entwicklungszusammenarbeit. Asien und Pazifik, S. 1-5.

Behrends, Jan C. (2010): Politische Führung in der Diktatur. In: BPB (Bundeszentrale für politische Bildung) (Hg.): Politische Führung, APuZ 2-3, S. 40-46.

Beuselinck, Ilse (2000): Internationalization and Doctoral Study: Some Reflections on Cross-Cultural Case Study Research. In: Pole, Christopher J. \& Robert G. Burgess (Hg.): Studies in Qualitative Methodology, Vol. 6: Cross-Cultural Case Study, Amsterdam, S. 83-94.

Beyerl, Katharina (2010): Der Klimawandel in der psychologischen Forschung. In: Voss, Martin (Hg.): Der Klimawandel. Sozialwissenschaftliche Perspektiven, Wiesbaden, S. 247-265.

Bhabha, Homi K. (1994): The Location of Culture, London.

Birkmann, Jörn (2006): Measuring vulnerability to promote disaster-resilient societies: Conceptual Frameworks and definitions. In: ders. (Hg.): Measuring Vulnerability to Natural Hazards. Towards Disaster Resilient Societies, S. 9-54, New York.

Birkmann, Jörn \& Teichman, Korinna (2009): Addressing the Challenge: Recommendations and Quality Criteria for Linking Disaster Risk Reduction and Adaptation to Climate Change, DKKV 38, Bonn.

Bittermann, Klaus, Rahmstorf, Stefan, Perrette, Mah'e \& Martin Vermeer (2013): Predictability of twentieth century sea-level rise from past data. Environ. Res. Lett. 8.

Block, Fred (2001): Introduction. In: Polanyi, Karl: The Great Transformation. The Political and Economic Origins of Our Time, Boston, S. xviii-xxxviii.

BMZ (Bundesministerium für wirtschaftliche Zusammenarbeit und Entwicklung) (2008): Auf den Weg in die eine Welt. Weißbuch zur Entwicklungspolitik. 13. entwicklungspolitischer Bericht der Bundesregierung, Bonn.

BMZ (2013): 14. entwicklungspolitischer Bericht der Bundesregierung. Weißbuch zur Entwicklungspolitik, Bonn.

Bogner, Alexander, Kastenhofer, Karen \& Helge Torgersen (2010): Inter- und Transdisziplinarität - Zur Einleitung in eine anhaltend aktuelle Debatte. In: dies. 
(Hg.): Inter- und Transdisziplinarität im Wandel? Neue Perspektiven auf problemorientierte Forschung und Politikberatung, Baden-Baden, S. 7-21.

Bohle, Hans-Georg (2001): Vulnerability and Criticality: Perspectives from Social Geography. In: IHDP Update 2, S. 1-5.

Bohle, Hans-Georg (2005): Soziales oder unsoziales Kapital? Das SozialkapitalKonzept in der Geographischen Verwundbarkeitsforschung. In: Geographische Zeitschrift 93, 2, S. 65-81.

Bohle, Hans-Georg, Etzhold, Benjamin \& Keck, Markus (2009): Resilience as agency. In: IHDP Update 2, S. 8-13.

Bourdieu, Pierre (1983a): Ökonomisches Kapital, kulturelles Kapital, soziales Kapital. In: Kreckel, Reinhard (Hg.): Soziale Ungleichheiten, Soziale Welt, Sonderband 2, Göttingen, S. 183-198.

Bourdieu, Pierre (1983²b): Zur Soziologie der Symbolischen Formen, Frankfurt am Main.

Bourdieu, Pierre (1987): Sozialer Sinn, Frankfurt am Main.

Brauch, Hans Günter (2008): Introduction: Globalization and Environmental Challenges: Reconceptualizing Security in the 21st Century. In: ders. (Hg.): Globalization and Environmental Challenges: Reconceptualizing Security in the 21st Century. Hexagon Series on Human and Environmental Security and Peace, Vol. 3, Berlin und Heidelberg, S. 27-43.

Briguglio, Lino (1995): Small Islands Developing States and Their Economic Vulnerabilities. In: World Development 23, 9, S. 1615-1632.

Brklacich, Mike, Chazan, May \& Hans-Georg Bohle (2010): Human Security, Vulnerability, and Global Environmental Change. In: Matthew, Richard A., Barnett, Jon, McDonald, Bryan \& Karen L. O’Brien (Hg.): Global Environmental Change and Human Security, S. 35-51.

Brown, Katrina, Adger, W. Neil, Tompkins, Emma, Bacon, Peter, Shim, David \& Kathy Young (2001): Trade-off analysis for marine protected area management. In: Ecological Economics 37, S. 417-434.

Brundtland, Gro Harlem (1987): Our Common Future. Report of the World Commission on Environment and Development. United Nations Report A/42/427, Oslo.

Burns, Alan (1963): Fiji, London.

Caron, Louis-Philippe \& Colin G. Jones (2007): Analysing present, past and future tropical cyclone activity as inferred from an ensemble of Coupled Global Climate Models. In: Tellus A 60, S. 80-96.

Castles, Stephen, De Haas, Hein \& Mark J. Miller (20145): The Age of Migration. International Population Movements in the Modern World. Basingstoke. 
Chambers, Robert \& Gordon Convey (1992): Sustainable rural livelihoods: practical concepts for the 21st century. IDS Discussion Paper 296, Brighton.

Chand, Satish (2011): The Political Economy of Sugar Rents in Fiji. In Duncan, Ron (Hg.): The Political Economy of Economic Reform in the Pacific, Manila, S. 345-366.

Chand, Savin S. \& Kevin J. E. Walsh (2009): Tropical Cyclone Activity in the Fiji Region: Spatial Patterns and Relationship to Large-Scale Circulation. In: Journal of Climate 22, S. 3877-3893.

CHS (Commission on Human Security) (2003): Human Security Now, New York.

Coleman, James S. (1988): Social Capital in the Creation of Human Capital. In: American Journal of Sociology 94, S. 95-120.

Connell, John (2013): Islands at Risk? Environments, Economies and Contemporary Change, Cheltenham.

Cooke, Bill \& Kothari, Uma $\left(2004^{3}\right)$ : The Case for Participation as Tyranny. In: dies. (Hg.): Participation: the new Tyranny? London, S. 1-15.

Dahl, Robert A. (1971): Polyarchy. Participation and Opposition, New Haven.

Därmann, Iris (2010): Theorien der Gabe zur Einführung, Hamburg.

Davis, Graham (2009): At home with Bainimarama. In: Fiji Sun (Zeitung), 08.05. 2009. http://www.fijisun.com.fj/main_page/view.asp?id=19246, 19.03.2010.

Dehaan, Leo \& Annelies Zoomers (2005): Development Geography at the Crossroads of Livelihood and Globalisation. In: Nijnhuis, Gery, Broekhuis, Annelet \& Guus van Westen (Hg.): Space and Place in Development Geography: Geographical Perspectives on Development in the 21st Century, S. 49-63, Amsterdam.

Deutscher, Eckard \& Hartmut Ihne (2010): Herausforderungen an die internationale Zusammenarbeit im 21. Jahrhundert. In: dies. (Hg.): ,Simplizistische Lösungen verbieten sich'. Zur internationalen Zusammenarbeit im 21. Jahrhundert. Festschrift zu Ehren von Professor Uwe Holtz, Baden-Baden, S. 11-14.

DFG (2014): Forschergruppe 1642: Landnahme, Beschleunigung, Aktivierung. Dynamik und (De-)Stabilisierung moderner Wachstumsgesellschaften, Jena. http://www.dfg.de/foerderung/programme/listen/projektdetails/index.jsp?id=1 89197227, 09.09.2014.

DfID (Department for International Development) (1999): Sustainable Livelihoods Guidance Sheets, London.

Diamond, Jared M. (20055): Kollaps. Warum Gesellschaften überleben oder untergehen, Frankfurt am Main.

Dickhardt, Michael (2001): Das räumliche des Kulturellen. Entwurf zu einer kulturanthropologischen Raumtheorie am Beispiel Fiji, Hamburg.

Dikau, Richard \& Jürgen Pohl (2007): „Hazards“: Naturgefahren und Naturrisiken. In: Gebhardt, Hans, Glaser, Rüdiger, Radke, Ulrich \& Paul Reuber (Hg.): Geogra- 
phie. Physische Geographie und Humangeographie, S. 1030-1075, Heidelberg und München.

Disaster Mitigation for Sustainable Livelihoods Programme (2008): Weathering the Storm. Participatory risk assessment for informal settlements, Kapstadt.

Dittrich, Christoph (2004): Bangalore: Globalisierung und Überlebenssicherung in Indiens Hightech-Kapitale. Studien zur Geographischen Entwicklungsforschung, 25, Saarbrücken

Dittrich, Christoph (2011): 10. Geographie. In: Niederberger, Andreas \& Philipp Schink (Hg.): Globalisierung. Ein interdisziplinäres Handbuch, Stuttgart, S. $180-187$.

Dörfler, Thomas, Graefe, Olivier, Detlef Müller-Mahn (2003): Habitus und Feld. Anregungen für eine Neuorientierung der geographischen Entwicklungsforschung auf der Grundlage von Bourdieus „Theorie der Praxis“. In: Geographica Helvetica 58,1, S. 11-23.

Ebeling, Ulrike, Kampfmeyer, Thomas, Nolting, Armin K. \& Roman Poeschke, (2007): Capacity Development in fragilen Staaten. In: BMZ (Hg.): Fragile Staaten - Beispiele aus der Entwicklungspolitischen Praxis, Baden-Baden, S. 337-351.

Ebert, Thomas (2012): Soziale Gerechtigkeit in der Krise, Lizenzausgabe für die Bundeszentrale für politische Bildung, Bonn.

Eckert, Andreas (2015): Geschichte der Entwicklungszusammenarbeit. In: bpb (Hg.): Entwicklungszusammenarbeit, APuZ 65, 7-9, S. 3-8.

Eisenstein, Charles (2011): Sacred Economics. Money, Gift \& Society in the Age of Transition, Berkeley.

Emanuel, Kerry (2005a): Increasing destructiveness of tropical cyclones over the past 30 years. In: Nature 436, S. 486-488.

Emanuel, Kerry (2005b): Emanuel replies. In: Nature 438, S. E13.

Engelkamp, Paul \& Friedrich L. Sell (2013): Einführung in die Volkswirtschaftslehre, Berlin \& Heidelberg.

Escobar, Arturo (1995): Encountering Development. The Making and Unmaking of the Third World, Princeton.

Escobar, Arturo (1996): Construction Nature. Elements for a post-structuralist political ecology. In: Futures, 28, 4, S. 325-343.

Escobar, Arturo (2011): Sustainability: Design for the pluriverse. In: Development 54, 2, S. 137-140.

Evans-Pritchard, Edward E. (1990): Vorwort. In: Mauss, Marcel: Die Gabe. Form und Funktion des Austauschs in archaischen Gesellschaften, Frankfurt am Main, S. 7-12. 
Faust, Heiko (1996): Verstädterung in Fiji. Besonderheiten in den Stadt-LandBeziehungen eines insularen pazifischen Entwicklungslandes. Schriftenreihe der Arbeitsgemeinschaft für Pazifische Studien e. V., 6, Aachen.

Faust, Heiko (2006): Fiji. In: Kreisel, Werner (Hg.): Mythos Südsee. Länderprofile Ozeaniens zu Wirtschaft und Gesellschaft, Hamburg, S. 39-44.

Faust, Heiko \& Johannes Winter (2005): Sensible Inselökonomien im Pazifik Politische Instabilität und ethnische Koexistenz als Determinanten für die Wirtschaftsentwicklung in Fiji. In: Waibel, Michael, Thimm, Tanja \& Kreisel, Werner $(\mathrm{Hg})$ : Inselökonomien. Schriftenreihe der Arbeitsgemeinschaft für Pazifische Studien e.V., 9, Bad Honnef, S. 119-133.

Faust, Jörg (2010): Wirkungsevaluierung in der Entwicklungszusammenarbeit. In: BPB (Hg.): Entwicklungspolitik, APuZ 10, S. 41-46.

FIBOS (Fiji Islands Bureau of Statistics) (2008): Census 2007 Results: Population size, growth, structure and distribution. 2007 Census of Population and Housing. Statistical News 45, 2008, Suva.

FIBOS (2009): Labour force, employment and housing. 2007 Census of Population and Housing. Statistical News 9, 2009, Suva.

FIBOS (2011): Report on the 2008-09 Household Income and Expenditure Survey for Fiji, Suva.

FIBOS (2012): Key Statistics: June 2012, Suva.

FIBOS (2014a): Gross Domestic Product by industry at constant basic prices of 2008, Suva. http://www.statsfiji.gov.fj/index.php/document-library/doc_down load/1127-contribution-to-gdp-at-constant-basic-prices-of-2008, 15.09.2014.

FIBOS (2014b): Balance of Trade Jun 2014, Suva. http://www.statsfiji.gov.fj/in dex.php/document-library/doc_download/1115-table-4-balance-of-trade, 20.09.2014.

FIBOS (2014c): Fijis Earnings from Tourism. Key Statistics December 2014, Suva. http://www.statsfiji.gov.fj/index.php/migration-a-tourism/10-migrationstatistics/migration-a-tourism/118-tourism-earnings-fjd-million, 05.05.2015.

Field, Colin D. (1995): Impact of expected climate change on mangroves. In: Hydrobiologica 295, S. 75-81.

Field, Michael (2007): The media and the spectre of the 2000 coup. In: Fraenkel, Jon \& Stewart Firth (Hg.): From Election to Coup in Fiji. The 2006 campaign and its aftermath, S. 174-184.

Fiji Ministry of Foreign Affairs Media Release \& SPREP (2014): Fijians elected to represent island States in global climate change bodies. Pressemitteilung, 13.12.2014, Lima. http://www.sprep.org/climate-change/fijians-elected-torepresent-island-states-in-global-climate-change-bodies, 13.02.2015. 
Fiji Ministry of Foreign Affairs Media Release (2012): Climate Change in School Curriculum. Pressemitteilung, 15.06.2012, Suva. http://www.foreignaffairs.gov.fj/ media-resources/media-release/77-climate-change-in-school-curriculum, 26.02.2015.

Fink, Michael (2010a): Vulnerability of small islands coastal villagers to natural hazards: Participatory livelihood analysis of Malawai village, Gau Island, Fiji. Vulnerability Mapping Working Paper No. 2, Bonn.

Fink, Michael (2010b): Verwundbarkeit ländlicher Küstenbewohner auf den Fidschi Inseln gegenüber Naturgefahren. Eine partizipative livelihood-Analyse, unveröffentlichte Magisterarbeit, Bonn.

Fink, Michael (2012): Yavirau: A traditional Fijian fish drive as an example of culturally embedded community development. In: Pacific News 38, S. 22-25.

Fink, Michael (2014): Die Auswirkungen einer luxuriösen Ferienhaussiedlung auf die dörflichen Gemeinschaften von Koro Island, Fidschi. In: Reeh, Tobias \& Gerhard Ströhlein (Hg.): Facetten der Landschaftsinterpretation und des Tourismus, Göttingen, S. 125-39.

Flick, Uwe (20077): Qualitative Sozialforschung. Eine Einführung, Reinbek bei Hamburg.

Fraenkel, Jon \& Stewart Firth (2009a): The enigmas of Fiji's good governance coup. In: Fraenkel, Jon, Firth, Stewart \& Brij V. Lal (Hg.): The 2006 Military Takeover in FIJI. A Coup to End All Coups? Canberra, S. 3-17.

Fraenkel, Jon \& Stewart Firth (2009b): Fiji's Coup Syndrome. In: Fraenkel, Jon, Firth, Stewart \& Brij V. Lal (Hg.): The 2006 Military Takeover in FIJI. A Coup to End All Coups? Canberra, S. 449-458.

Freud, Sigmund (1913/1991): Totem und Tabu. Einige Übereinstimmungen im Seelenleben der Wilden und der Neurotiker, Frankfurt am Main.

Freytag, Tim (2014): Raum und Gesellschaft. In: Lossau, Julia, Freytag, Tim \& Roland Lippuner (Hg.): Schlüsselbegriffe der Kultur- und Sozialgeographie, Stuttgart, S. 12-24.

Gebhardt, Hans, Glaser, Rüdiger, Radke, Ulrich \& Paul Reuber (2011a) (Hg.): Teil III Arbeitsmethoden der Geographie. In: dies. (Hg.): Geographie. Physische Geographie und Humangeographie, Heidelberg, S. 84-224.

Gebhardt, Hans, Glaser, Rüdiger, Radke, Ulrich \& Paul Reuber (2011b): Räumliche Maßstäbe und Gliederungen - von global bis lokal. In: dies. (Hg.): Geographie. Physische Geographie und Humangeographie, Heidelberg, S. 13-35.

Geertz, Clifford (1983): Dichte Beschreibung. Beiträge zum Verstehen kultureller Systeme, Frankfurt am Main. 
Gemenne, François Barnett, Jon, Adger, W. Neil \& Geoffrey D. Dabelko (2014): Climate and security: evidence, emerging risks, and a new agenda. In: Climatic Change 123, S. 1-9.

Geoffrey D. Dabelko

Gero, Anna, Meheux, Kristie \& Dale Dominey-Howes (2010): Disaster risk reduction and climate change adaptation in the Pacific: The challenge of integration. ATRC-NHCL Miscellaneous Report 4, Sydney.

Giddens, Anthony (1988): Die Konstitution von Gesellschaft. Grundzüge einer Theorie der Strukturierung, Frankfurt am Main.

Giddens, Anthony (19993): Konsequenzen der Moderne, Frankfurt am Main.

Gießen, Bernhard \& Robert Seyfert (2013): Kollektive Identität. In: bpb (Hg.): Gesellschaftliche Zusammenhänge, APuZ 63, 13-14, S. 39-43.

Gopal, Avinesh (2014): 'Give up Lau'. In: Fiji Times Online (Zeitung), 03.07.2014. http://www.fijitimes.com/story.aspx?id=273246, 8.07.2014.

Gördes, Hugo (1916): Die Arbeiterfrage in der deutschen Südsee unter Berücksichtigung der englischen Fidschi-Inseln. Ein Beitrag zur Lehre von der Volkswirtschaft in tropischen Ländern, Karlsruhe.

Govan, Hugh, Tawake, Alifereti; Korovulavula, Isoa \& Seini Tawakelevu (2009): Summary analysis of site support costs for Fiji Locally Managed Marine Area (FLMMA). Institute of Applied Sciences Technical Report.

Graeffe, Eduard (1868): Reisen im Innern der Insel Viti-Levu, Zürich.

Groome Wynne, Barbara (2007): Social Capital and Social Economy in SubNational Islands Jurisdictions. In: Island Studies Journal 2, 1, S. 115-132.

Grootaert, Christiaan, Narayan, Deepa, Nyhan Jones, Veronica \& Michael Woolcock (2004): Measuring Social Capital. An Integrated Questionnaire. World Bank working paper No. 18.

GTAI (2014): Wirtschaftsdaten kompakt: Deutschland. Stand: Mai 2014, Bonn (Germany Trade \& Invest, Gesellschaft für Außenwirtschaft und Standortmarketing $\mathrm{mbH})$.

Haller, Dieter $\left(2010^{2}\right)$ : dtv-Atlas Ethnologie, München.

Harriss, John (2002): Depoliticizing Development. The World Bank and Social Capital, London.

Harvey, David (2001): Globalization and the "Spatial Fix". In: geographische revue, 3, 2, S. 23-30.

Hau'ofa, Epeli (1993): Our Sea of Islands. In: Waddell, Eric, Naidu, Vijay \& Epeli Hau'ofa (Hg.): A New Oceania. Rediscovering Our Sea of Islands, Suva, S. 2-16.

Hauser-Schäublin, Brigitta (2003): Teilnehmende Beobachtung. In: Beer, Bettina (Hg.): Methoden und Techniken der Feldforschung, Berlin, S. 33-54. 
Have, Paul ten (2004): Understanding Qualitative Research and Ethnomethodology, London.

Hedtke, Reinhold (2014): Wirtschaftssoziologie, Konstanz.

Heidemann, Frank (2011): Ethnologie. Eine Einführung, Göttingen.

Hermann, Elfriede (2007): Communicating with Transculturation. In: DouaireMarsaudon, Françoise (Hg.): Pacific Challenges: Questioning Concepts, Rethinking Conflicts. Special Issue of Journal de la Société des Océanistes 125, 2, S. $257-260$.

Herrmann-Pillath, Carsten (2011): Revisiting the Gaia hypothesis: Maximum Entropy, Kauffman's 'Fourth Law' and physiosemeiosis. Frankfurt School of Finance \& Management, Working paper No. 160.

Heuer, Wolfgang (2006): Politik und Verantwortung. In: BPB (Hg.): Hannah Arendt, APuZ 56, 39, S. 8-15.

Hickey, Samuel \& Giles Mohan (2004): Towards Participation as Transformation: Critical Themes and Challenges for a Post-Tyranny Agenda. In: dies. (Hg.): Participation: from tyranny to transformation? Exploring new approaches to participation in development, London \& New York, S. 5-19.

Hobbes, Thomas (1651/200412): Der Staat als Instrument eines aufgeklärten Egoismus. In: Hörster, Norbert (Hg.): Klassische Texte der Staatsphilosophie, München, S. 109-133.

Hoegh-Guldberg, O., Mumbay, P. J., Hooten A. J., Steneck, R. S., Greenfield, P., Gomez, E., Harvell, C. D., Sale, P. F. , Edwards, A. J., Caldeira, K., Knowlton, N., Eakin, C. M., Iglesias-Prieto, R., Muthiga, N., Bradbury, R. H., Dubi, A. \& M. E. Hatziolos (2007): Coral Reefs Under Rapid Climate Change and Ozean Acidification. In: Science 318, S. 1737-1742.

Honer, Anne (2007): Lebensweltanalyse in der Ethnographie. In: Flick, Uwe; Kardoff, Ernst von \& Ines Steinke (Hg.): Qualitative Forschung, Reinbek bei Hamburg, S.194-204.

Holtz, Uwe (2011): Entwicklungspolitisches Glossar. Stichwörter zur Entwicklungs- und Eine Welt-Politik, Bonn. http://www.uni-bonn.de/ uholtz/virtapparat/EP_Glossar.pdf, 22.08.2013.

IPCC (Intergovernmental Panel on Climate Change) (2007a): Climate Change 2007, Contribution of Working Group I to the Fourth Assessment Report of the Intergovernmental Panel on Climate Change, Cambridge, New York.

IPCC (2007b): Climate Change 2007, Synthesis Report: A Contribution of Working Groups I, II, and III to the Fourth Assessment Report of the Intergovernmental Panel on Climate Change, Cambridge, New York. 
IPU (Interparlamentarische Union) (1997): Results. 98th conference and related meetings of the Inter-Parliamentary Union, Kairo. http://www.ipu.org/confe/98.pdf, 14.09.2010.

IFRC (2007a): Vulnerability and Capacity Assessment (VCA) toolbox with reference sheets. International Federation of Red Cross and Red Crescent Societies, Genf.

IFRC (2007b): How to do a VCA. A practical step-by-step guide for Red Cross Red Crescent staff and volunteers. International Federation of Red Cross and Red Crescent Societies, Genf.

Jagmohan, Mala (1993): From the editor's desk. An ill wind blows some good. In: Pacific Islands Monthly 63, 2, S. 4.

Jolly, Margaret (1992): Custom and the Way of the Land: Past and Present in Vanuatu and Fiji. In: Oceania62, 4, S. 330-354.

Jones, Peter J.S. (2002): Marine protected area strategies: issues, divergences and the search for middle ground. In: Reviews in Fish Biology and Fisheries 11, S. $197-$ 216.

Kaly, Ursula L., Pratt, Craig R. \& Russel Howorth (2002): Towards Managing Environmental Vulnerability in Small Islands Developing States (SIDS). SOPAC Miscellaneous Report 461.

Kay, Alan (2005): Social Capital, the Social Economy and Community Development. In: Community Development Journal 41, 2, S. 160-173.

Karakayali, Serhat (2013): Kosmopolitische Solidarität. In: BPB (Hg.): Gesellschaftliche Zusammenhänge, APuZ 63, 13-14, S. 21-26.

Keck, Markus \& Patrick Sakdapolrak (2013): What is social resilience? Lessons learned and ways forward. In: Erdkunde, 67, 1, S. 5-19.

Keck, Verena (2003): Interdisziplinäre Projekte und Teamarbeit. In: Beer, Bettina (Hg.): Methoden und Techniken der Feldforschung, Berlin, S. 203-223.

Kleidon, Axel (2010): Life, Hierarchy, and the Thermodynamic Machinery of Planet Earth. In: Physics of Life Reviews 7, S. 424-460.

Klingebiel, Stephan (2015): Zukunft der Entwicklungszusammenarbeit. In: bpb (Hg.): Entwicklungszusammenarbeit, APuZ 65, 7-9, S. 16-22.

Korf, Benedikt (2009): Partizipation als Ausnahmezustand. In: Geographica Helvetica 64, 2, S. 98-105.

Korf, Benedikt \& Eberhard Rothfuss (2016): Nach der Entwicklungsgeographie. In: Freitag, Tim, Gebhardt, Hans, Gerhard, Ulrike \& Doris Wastl-Walter (Hg.): Humangeographie kompakt. Heidelberg und Berlin, S. 163-183.

Kotoisuva, Edwina (2010): Incidence, forms and prevalence of violence against women in the Pacific. In: Narsey, Wadan; Sachs Robertson, Annette, Prasad, Biman Chand, Seniloli, Kesaia, Jongstra, Eduard, \& WordWorks Fiji (Hg.): Popu- 
lation and Development in the Pacific Islands. Accelerating the ICPD Programme of Action at 15, Suva, S. 238-242.

Kretzenbacher, Heinz L. (1995): Wie durchsichtig ist die Sprache der Wissenschaften? In: Kretzenbacher, Heinz L. \& Harald Weinrich (Hg.): Linguistik der Wissenschaftssprache, Berlin, S. 15-40.

Kumar, Somesh (2002): Methods for Community Participation. A Complete Guide for Practitioners, London.

Kuschel, Rolf, Takiika, 'Angikinui Francis T. \& Kiu 'Angiki (2005): Alcohol and Drug Use in Honiara, Solomon Islands. A Cause for Concern. In: Marsella, Antony J., Austin, Ayda Aukahi \& Bruce Grant (Hg.): Social Change and Psychosocial Adaptation in the Pacific Islands. Cultures in Transition, Honolulu, S. 211-254.

Landsea, Cristopher W. (2005): Hurricanes and Global Warming. In: Nature 438, S. E11-E13.

Landsea, Christopher W., Harper, Bruce A., Hoarau, Karl \& John A. Knaff (2006): Can We Detect Trends in Extreme Tropical Cyclones? In: Science 313, S. $452-$ 454.

Lal, Brij V. (2003): Heartbreak Islands: Reflections on Fiji in transition. In: Asia Pacific Viewpoint 44, 3, S. 335-350.

Lal, Brij V. (2012): From Common Roll to Consociationalism: The Political Trajectory of the Fiji Indians. Keynote Speech im Rahmen der Konferenz "India beyond India - Debating Communalism and Belonging", 24.-26.5.2012, 24.5.2012, Göttingen.

Lata, Shalini \& Patrick D. Nunn (2011): Misperceptions of climate-change risk as barriers to climate-change adaptation: a case study from the Rewa Delta, Fiji. In: Climatic Change 110, 1-2, S. 169-186.

Lauer Matthew \& Shankar Aswani (2010): Indigenous Knowledge and Long-term Ecological Change: Detection, Interpretation, and Responses to Changing Ecological Conditions in Pacific Islands Communities. In: Environmental Management 45, S. 985-997.

Lauth, Hans-Joachim (2002): Totalitarismus-Autoritarismus-Demokratie. In: ders. (Hg.): Vergleichende Regierungslehre, Wiesbaden, S. 105-130.

Lawson, Stephanie (2004): Nationalism versus constitutionalism in Fiji. In: Nations and Nationalism 10, 4, S. 519-538.

Lefebvre, Henri (1972): Das Alltagsleben in der modernen Welt, Frankfurt am Main.

Leggewie, Claus \& Harald Welzer $\left(2011^{2}\right)$ : Das Ende der Welt, wie wir sie kannten. Klima, Zukunft und die Chancen der Demokratie. Lizenzausgabe für die Bundeszentrale für politische Bildung, Bonn. 
Leichenko, Robert M. \& Karen O’Brien (2008): Environmental Change and Globalization. Double Exposures, Oxford.

Lewai, Vasemaca (2010): Population ageing in Fiji: current trends and challenges. In: Narsey, Wadan, Sachs Robertson, Annette, Prasad, Biman Chand, Seniloli, Kesaia, Jongstra, Eduard, \& WordWorks Fiji (Hg.): Population and Development in the Pacific Islands. Accelerating the ICPD Programme of Action at 15, Suva, S. 85-94.

Lewis, Vaughan A. (2009): Foreword: Studying Small States over the Twentieth into the Twenty-first Centuries. In: Cooper, Andrew F. \& Timothy M. Shaw, (Hg.): The Diplomacies of Small States. Between Vulnerability and Resilience, London, S. vii-xv.

Lossau, Julia (2014): Kultur und Identität. In: Lossau, Julia, Freytag, Tim \& Roland Lippuner (Hg.): Schlüsselbegriffe der Kultur- und Sozialgeographie, Stuttgart, S. 25-37.

Lovelock, James (1991): Das Gaia-Prinzip. Die Biographie unseres Planeten, München.

Luhmann, Niklas (2010): Politische Soziologie, Frankfurt am Main.

Lund, Christian (2014): Of What is This a Case?: Analytical Movements in Qualitative Social Science Research. In: Human Organization 73, 3, S. 224-234.

Machiavelli, Niccolò (ca. 1513/200412): Realpolitische Bedingungen der Ausübung staatlicher Macht. In: Hörster, Norbert (Hg.): Klassische Texte der Staatsphilosophie, München, S. 98-108.

Malinowski, Bronisław (1922): The Argonauts of the Western Pacific, London.

Mander, Jerry \& Edward Goldsmith (Hg.) (2002²): Schwarzbuch Globalisierung, München.

Mannheim, Karl (1929/1952): Ideologie und Utopie, Frankfurt am Main.

Marchart, Oliver (2008): Cultural Studies. Lizenzausgabe für die Bundeszentrale für politische Bildung, Konstanz.

Mattissek, Annika, Pfaffenbach, Carmella \& Paul Reuber $\left(2013^{2}\right)$ : Methoden der empirischen Humangeographie, Braunschweig.

Mauss, Marcel (1923-25/1990): Die Gabe. Form und Funktion des Austauschs in archaischen Gesellschaften, Frankfurt am Main.

Mataki, Melchior, Koshy, Kanayathu \& Veena Nair (2008): Top-Down, Bottom Up: Main-streaming Adaptation in Pacific Islands Townships. In: Leary, Neil, Adejuvon, James, Barros, Vicente, Burton, Ian, Kulkarni, Jyoti \& Rodel Lasco (Hg.): Climate Change and Adaptation, London, S. 264-278.

Mayring, Philipp (20025): Einführung in die Qualitative Sozialforschung, Weinheim und Basel. 
McFadzien, Diane, Areki, Francis, Biuvakadua, Tarai \& Monifa Fiu (2009): Climate Witness Community Toolkit. WWF South Pacific - Climate Change Team, Suva.

McQuiston, Chris, Parrado, Emilio A., Olmos-Muñiz, Julio César \& Alejandro M. Bustillo Martinez (2005): Community-Based Participatory Research and Ethnography. The Perfect Union. In: Israel, Barbara A., Eng, Eugenia, Schulz, Amy J. \& Edith A. Parker (Hg.): Methods in Community-Based Participatory Research for Health, San Francisco, S. 210-229.

Meier Kruker, Verena \& Jürgen Rauh (2005): Arbeitsmethoden der Humangeographie, Darmstadt.

Melber, Henning (1992): Der Weißheit letzter Schluß. Rassismus und kolonialer Blick, Frankfurt am Main.

Merkel, Wolfgang (1999): Systemtransformation, Opladen.

Merkel, Wolfgang, Puhle, Hans-Jürgen, Croissant, Aurel, Eicher, Claudia \& Peter Thiery (2003): Defekte Demokratie, Opladen.

Morcos, Karim \& Anne F. Röder (2007): Die Arbeit der OECD zu fragilen Staaten und Situationen. In: BMZ (Hg.): Fragile Staaten - Beispiele aus der Entwicklungspolitischen Praxis, Baden-Baden, S. 289-299.

Mückler, Hermann (1998): Fidschi. Zwischen Tradition und Transformation. Koloniales Erbe, Häuptlingstum und ethnische Heterogenität als Herausforderung für die Zukunft, Frankfurt am Main.

Mückler, Hermann (2007): Invisible Lines - on the Difficulties of Drinking. Unsichtbare Linien - oder: das komplizierte Trinken. In: Journal of Comparative Cultural Studies in Architecture 2007, 1, S. 13-20.

Müller, Franziska \& Aram Ziai (2015): Eurozentrismus in der Entwicklungszusammenarbeit. In: bpb (Hg.): Entwicklungszusammenarbeit, APuZ 65, 7-9, S. 815.

Müller, Martin (2012): Mittendrin statt nur dabei: Ethnographie als Methodologie in der Humangeographie. In: Geographica Helvetica 67, 3, S. 179-184.

Nabobo-Baba, Unaisi (2006): Knowing and learning. An indigenous Fijian approach, Suva.

Naleba, Mere (2014): Parties reject result. In: Fiji Times Online, 19.09.2014. http:/ /www.fijitimes.com/story.aspx?id=280658, 29.09.2014.

Narayan, Paresh Kumar \& Biman Chand Prasad (2003): Fiji's Sugar, Tourism and Garment Industries: A Survey of Performance, Problems and Potentials. In: Fiji Institute of Applied Studies (Hg.): Fijian Studies 1, 1, S. 3-27.

Nasiko, Repeka (2012): Maintain chiefs, leaders rights. In: Fiji Times Online, 01.09.2012. http://www.fijitimes.com/story.aspx?id=210682, 8.7.2014.

Nayacakalou, Rusiate R. (1978): Tradition and Change in the Fijian Village, Suva. 
NEOC (National Emergency Operation Center) (2010a): Situation Report. Tropical Cyclone Tomas. DISMAC, SITREP 18, 21.03.2010, Suva.

NEOC (2010b): Report on TC "Tomas". Präsentation von DOBUI, Pajiliai, NDMO (National Disaster Management Office) während PHT-Meeting (Pacific Humanitarian Team), 12.04.2010, Suva.

Neubert, Dieter (2001): Entwicklung unter dem Mikroskop. Der akteursorientierte Ansatz. In: E+Z - Entwicklung und Zusammenarbeit 7/8, Frankfurt am Main, S. 216-219.

Neubert, Dieter, Neef, Andreas \& Friedrichsen, Rupert (2008): Interaktive Methoden: Erfahrungen mit der Verwendung von „Participatory Rural Appraisal“ (PRA) in der Forschung. In: Cappai, Gabriele (Hg.): Forschen unter Bedingungen kultureller Fremdheit, Wiesbaden, S. 95-126.

Newland, Lynda (2007): The role of the Assembly of Christian Churches in Fiji in the 2006 election. In: Fraenkel, Jon \& Stewart Firth (Hg.): From election to coup in Fiji. The 2006 campaign and its aftermath, Canberra,S. 300-314.

Newland, Lynda (2009): Religion and politics: The Christian churches and the 2006 coup in Fiji. In: Fraenkel, Jon, Firth, Stewart \& Brij V. Lal (Hg.): The 2006 Military Takeover in FIJI. A Coup to End All Coups? Canberra, S. 187-207.

NOAA (2013): Solomon Islands Tsunami, February 6, 2013. Main Event Page. US National Oceanic and Atmospheric Administration Center for Tsunami Research. http://nctr.pmel.noaa.gov/solomon20130206/, 19.11.2014.

Nunn, Patrick D. (1998): Pacific Islands Landscapes. Landscape and geological development of southwest Pacific Islands, especially Fiji, Samoa and Tonga, Suva.

Nunn, Patrick D. (2007): Climate, environment and society in the pacific during the last millennium, Amsterdam.

Nuscheler, Franz (20055): Entwicklungspolitik, Lizenzausgabe für die Bundeszentrale für politische Bildung, Bonn.

Nuscheler, Franz (2008): Die umstrittene Wirksamkeit der Entwicklungszusammenarbeit. Institut für Entwicklung und Frieden, INEF-Report 93, Duisburg.

O’Brien, Karen \& Johanna Wolf, (2010): A values-based approach to vulnerability and adaptation to climate change. In: Wiley Interdisciplinary Reviews: Climate Change 1, 2, S. 232-242.

OCHA (UN Office for the Coordination of Humanitarian Affairs) (2010): Situation Report TC Tomas, Situation Report 6, 12.04.2010, Suva.

Ortiz, Fernando (1947/1995): Cuban Counterpoint. Tobacco and Sugar, Durham und London.

Ostrom, Elinor (2000): Collective Action and the Evolution of Social Norms. In: The Journal of Economic Perspectives, 14, 3, S. 137-158 
PACC (Pacific Adaptation to Climate Change) (2009): Fiji Islands. Report of InCountry Consultations. http://www.sprep.org/att/publication/000668_FijiRepo rt_NationalPACCReport_Final.pdf, 07.09.2014.

Palonen, Kari (1998): Das 'Webersche Moment'. Zur Kontingenz des Politischen, Obladen.

Peet, Richard \& Elaine Hartwick, $\left(2009^{2}\right)$ : Theories of Development. Contentions, Arguments, Alternatives, New York.

Peluso, Nancy Lee \& Christian Lund (2011): New frontiers of land control: Introduction. In: The Journal of Peasant Studies, 38, 4, S. 667-681.

Pielke, Roger A. (2005): Are there trends in hurricane destruction? In: Nature 438, S. E11.

Pole, Christopher J. (2000): Case, Culture, Curriculum and Educational Change: Reflections on Cross-Cultural Case Study in England and Japan. In: Pole, Christopher J. \& Robert G. Burgess (Hg.): Studies in Qualitative Methodology, Vol. 6: Cross-Cultural Case Study, Amsterdam, S. 95-111.

Polanyi, Karl (1944/2001): The Great Transformation. The Political and Economic Origins of Our Time, Boston.

Pratt, Garett (2003): Discovering new faces of PRA. In: Cornwall, Andrea und Garett Pratt (Hg.): Pathways to Participation. Reflections on PRA, London, S. 164-169.

Prasad, Naren (2009): Small but Smart: Small States in the Global System. In: Cooper, Andrew F. \& Timothy M. Shaw (Hg.): The Diplomacies of Small States. Between Vulnerability and Resilience, London, S. 41-64.

Przyrembel, Alexandra (2011): Verbote und Geheimnisse: Das Tabu und die Genese der europäischen Moderne, Frankfurt am Main.

Putnam, Robert (1993): Making democracy work: Civic traditions in modern Italy, Princeton.

Putnam, Robert (1995): Bowling Alone: America's Declining Social Capital. In: Journal of Democracy 6, 1, S. 65-78.

Rahmstorf, Stefan \& Hans Joachim Schellnhuber (20076): Der Klimawandel. Diagnose, Prognose, Therapie, München.

Ratuva, Steven (2007): The pre-election 'cold war': The role of the Fiji military during the 2006 elections. In: Fraenkel, Jon \& Stewart Firth (Hg.): From Election to Coup in Fiji. The 2006 campaign and its aftermath, S. 26-45.

Ratuva, Steven (2013): Politics of preferential development. Trans-global study of affirmative action and ethnic conflict in Fiji, Malaysia and South Africa, Canberra.

Ravuvu, Asesela (1983): Vaka i Taukei. The Fijian Way of Life. Suva.

Ravuvu, Asesela (1987): The Fijian Ethos, Suva. 
Reddy, Mahendra (2000): Natural Disasters and the Island Economies: an examination of the economic cost of natural disasters in Fiji. In: South Pacific Study 21, 1, S. 33-43.

Reddy, Mahendra, Mohanty, Manoranjan \& Vijay Naidu (2004): Economic Cost of Human Capital Loss from Fiji: Implications of Sustainable Development. In: The International Migration Review, 38, 4, S. 1447-1461.

Reed, Alexander Wyclif \& Alice Inez Hames (1967): Myths and Legends of Fiji and Rotuma, Wellington.

Reeh, Tobias \& Kreisel,Werner (2002): Meereserwärmung, Korallenbleiche und Tourismus im Pazifik. In: Pacific News 19, S. 23-25.

Reithofer, Hans (2009): Verkörperte Feldforschung als Ethnologe und Missionar. In: Hermann, Elfriede, Klenke, Karin \& Michael Dickhardt (Hg.): Form, Macht, Differenz: Motive und Felder ethnologischen Forschens, Göttingen, S. 357-370.

Robertson, Roland (1995): Glocalization: Time-Space and HomogeneityHeterogeneity. In: Featherstone, Mike, Lash, Scott \& Roland Robertson (Hg.): Global Modernities, London, S. 25-44.

Rogall, Holger $\left(2013^{2}\right)$ : Volkswirtschaftslehre für Sozialwissenschaftler. Einführung in eine zukunftsfähige Wirtschaftslehre, Wiesbaden.

Rothfuß, Eberhard (2012): Exklusion im Zentrum: die brasilianische Favela zwischen Stigmatisierung und Widerständigkeit, Bielefeld.

Rüb, Friedbert W. (2014): Die Politik in der politischen Gesellschaft. Eine ideengeschichtliche Spurensuche. In memoriam Michael Th. Greven (07.03.194707.07.2012). In: PVS 55, 2, S. 356-386.

Sahlins, Marschall (1981): The Stranger-King: Or Dumézil among the Fijians. In: The Journal of Pacific History, 16, 3, S. 107-132.

Said, Edward W. (1978): Orientalism, New York.

Salleh, Ariel (2010): From Metabolic Rift to "Metabolic Value": Reflections on Environmental Sociology and the Alternative Globalization Movement. In: Organization \& Environment, 23, 2, S. 205- 219.

Sanders, Robert T. (1963): Interlude. In: Burns, Alan: Fiji, London, S. 149-173.

Sano, Yae (2008a): Social and institutional arrangements in coastal common pool resource systems: preliminary results of a study of community-based coastal management in Fiji. In: Tropics, 17, 4, S.295-314.

Sano, Yae (2008b): The role of social capital in a common property resource system in coastal areas: A case study of community-based coastal resource management in Fiji. In: SPC Traditional Marine Resource Management and Knowledge Information Bulletin, 24, S. 19-32.

Scarr, Deryck (1984): Fiji. A short history, Sydney und London. 
Schaeffer, Michiel, Hare, William, Rahmstorf, Stefan \& Martin Vermeer (2012): Long-term sea-level rise implied by $1.5^{\circ} \mathrm{C}$ and $2^{\circ} \mathrm{C}$ warming levels. In: Nature Climate Change, 2, 12, S. 867-870.

Schmelzer, Matthias (2014): Gutes Leben statt Wachstum. Degrowth, Klimagerechtigkeit, Subsistenz - Eine Einführung in die Begriffe und Ansätze der Postwachstumsbewegung. In: Le Monde diplomatigue (Hg.): Atlas der Globalisierung. Weniger wird mehr. Der Postwachstumsatlas (Exklusive Vorschau), S. 16-21.

Schmitt, Carl (1932/19636): Der Begriff des Politischen. Text von 1932 mit einem Vorwort von drei Corollarien, Berlin.

Sen, Amartya (1979): Equality of What? In: The Tanner Lecture of Human Values, S. 195-220.

Sen, Amartya (1981): Poverty and Famines: An Essay on Entitlement and Deprivation, Clarendon, Oxford.

Sen, Amartya (2004a): How Does Culture Matter? In: Rao, Vijayendra \& Michael Walton (Hg.): Culture and Public Action, Stanford, S. 37-58.

Sen, Amartya (2004b): Rationality and Freedom, Cambridge \& London.

Sen, Amartya (2007): Die Identitätsfalle. Warum es keinen Krieg der Kulturen geben kann. Lizenzausgabe für die Bundeszentrale für politische Bildung, Bonn.

Sen, Amartya (2010): Die Idee der Gerechtigkeit. Lizenzausgabe für die Bundeszentrale für politische Bildung, Bonn.

Senft, Gunter (2003): Zur Bedeutung der Sprache für die Feldforschung. In: Beer, Bettina (Hg.): Methoden und Techniken der Feldforschung, Berlin, S. 55-70.

Shameem, Nazhat (2005): In the High Court of Fiji at Suva. Appellate Jurisdiction. Crim. App. Nos: HAA 67, 70 \& 73 of 2005S; 14. November 2005, Suva.

Sitter-Liver, Beat (2012): Zum Status der Kreaturen - eine politische Streitfrage. In: ZfP 59, 4, S. 376-392.

Slocum, Nikki (2003): Participatory Methods Toolkit. A practitioner's manual, o.O.

Sofer, Michael (2009): Twenty years of change in the Fijian periphery: The case of the Kadavu Island. In: Singapore Journal of Tropical Geography 30, S. 343-357.

SOPAC (2009): Relationship between natural disasters and poverty: A Fiji case Study. A report prepared for the United Nations International Strategy for Disaster Reduction Secretariat's 2009 Global Assessment Report on Disaster Reduction, SOPAC Miscellaneous Report 678.

SPC (2013): Pacific Island Populations 2013, Secretariat of the Pacific Community, Nouméa, Neukaledonien.

SPC (2014a): Fiji updates school curricula with SPC-GIZ support, 22 März 2014, Secretariat of the Pacific Community (SPC), Suva. http://www2008.spc.int/en/ links-and-resources/1628-fiji-updates-school-curricula-with-spc-giz-support-.html, 16.02.2015. 
SPC (2014b): Education students build their understanding of climate change adaptation, 5 November 2014, Sabeto, Secretariat of the Pacific Community. http://www.spc.int/en/component/content/article/216-about-spc-news/1910education-students-build-their-understanding-of-climate-change-adaptation-html, 25.11.2014.

Spradley, James P. (1979): The Ethnographic Interview, New York.

Spradley, James P. (1980): Participant Observation, Orlando.

Steinebach, Stefanie (2009): „Da willst du wirklich hin? Als Frau, so ganz allein?“: Geschlecht im Feld. In: Hermann, Elfriede, Klenke, Karin \& Michael Dickhardt (Hg.): Form, Macht, Differenz: Motive und Felder ethnologischen Forschens, Göttingen, S. 371-385.

Steinke, Ines (2007): Gütekriterien qualitativer Forschung. In Flick, Uwe; Kardoff, Ernst von \& Ines Steinke (Hg.): Qualitative Forschung, Reinbek bei Hamburg, S.319-331.

Stiglitz, Joseph E. (2001): Foreword. In Polanyi, Karl: The Great Transformation. The Political and Economic Origins of Our Time, Boston, S. vii-xvii.

Sutton, Philip W. (2004): Nature, Environment and Society, New York.

Taylor, Robin (2005): Fiji's Move into the 21st Century. In: Marsella, Anthony J., Austin, Ayda Aukahi \& Bruce Grant (Hg.): Social Change and Psychosocial Adaptation in the Pacific Islands. Cultures in Transition, Honolulu, S. 107-132.

Terry, James P. (2007): Tropical Cyclones. Climatology and Impacts in the South Pacific, Suva.

Thywissen, Katharina (2006): Core terminology of disaster reduction: A comparative glossary. In: Birkmann, Jörn (Hg.): Measuring Vulnerability to Natural Hazards. Towards Disaster Resilient Societies, S. 448- 496, New York.

Thaman, Randolph R. (1982): Hurricane Isaac and Tonga: A natural or cultural disaster? In: Journal of the School of Social and Economic Development 3, 8, S. 22-35.

Tiffany, Martin (1993a): Disaster. Fiji picks up the pieces after the onslaught of Cyclone Kina. In: Pacific Islands Monthly 63, 2, S. 22-25.

Tiffany, Martin (1993b): Fiji radar fails. In: Pacific Islands Monthly 63, 2, S. 23.

Toren, Christina (1988): Making the Present, Revealing the Past: The Mutability and Continuity of Tradition as Process. In: Man, New Series, 23, 4, S. 696-717.

Toren, Christina (1999): Mind, Materiality and History. Explorations in Fijian Ethnography, London.

Trnka, Susanna (2011): Re-mythologizing the State: Public Security, 'the Jesus Strategy' and the Fiji Police. In: Oceania, 81, 1, S. 72-87.

Tröger, Sabine (2003): Akteure in ihrer Lebensgestaltung (livelihood) zu Zeiten sozialer Transformation - Theoretische Überlegungen und ihre Anwendung auf 
das Beispiel von Landnutzungskonflikten in Tansania. In: Geographica Helvetica 58, 1, S. 24-34.

Tuiwavu, Tevita (2012): No more GCC. In: Fiji Times Online, 15.03.2012. http://www.fijitimes.com/print.aspx?id=196039, 13.05.2014.

Turner, James W. (1986): "The Water of Life": Kava Ritual and the Logic of Sacrifice. In: Ethnology, 25, 3, S. 203-214.

Ukowitz, Martina (2014): Auf dem Weg zu einer Theorie transdisziplinärer Forschung. In: GAIA 23, 1, S. 19-22.

UN (United Nations) (1948): Universal Declaration of Human Rights http://www.un.org/en/documents/udhr/, 09.05.2010.

UN (2000): United Nations Millennium Declaration. http://www.un.org/millenni um/declaration/ares552e.htm, 07.08.2013.

UN Department of Economic and Social Affairs (2014): World Urbanization Prospects: The 2014 Revision, Highlights.

UN Statistics Division (2015): Population by sex and urban/rural residence. http:/ / data.un.org/Data.aspx?q=fiji + datamart $\% 5 \mathrm{bPOP} \% 5 \mathrm{~d} \& \mathrm{~d}=\mathrm{POP} \& \mathrm{f}=$ tableC ode $\% 3 a 1 \% 3$ bcountryCode $\% 3 a 242,24.03 .2013$.

UNDP-HDR (2014a): Human Development Report 2014. Sustaining Human Progress: Reducing Vulnerabilities and Building Resilience, New York.

UNDP-HDR (2014b): Table 2: Human Development Index trends, 1980-2013. http://hdr.undp.org/en/content/table-2-human-development-index-trends-19802013, 13.08.2014.

UNDP-HDR (2011): Human Development Report 2011. Sustainability and Equity: A Better Future for All, New York.

Varian, Hal R. (20046): Grundzüge der Mikroökonomik, München.

Veitayaki, Joeli (1995): Fisheries development in Fiji: the quest for sustainability, Suva.

Veitayaki, Joeli (2006): Caring for the Environment and the Mitigation of Natural Extreme Events in Gau, Fiji Islands: A Self-help Community Initiative. In: Island Studies Journal 1, 2, S. 239-252.

Veitayaki, Joeli \& Michael Fink (2012): Disaster Risk Preparedness on Gau Island, Fiji: Report of a workshop in Lamiti, Gau Island: January 9th - 13th, 2012, Suva.

Verne, Julia (2012): Ethnographie und ihre Folgen für die Kulturgeographie: eine Kritik des Netzwerkkonzepts in Studien zu translokaler Mobilität. In: Geographica Helvetica 67, 3, S. 185-194.

Villagrán De León, Juan Carlos (2006): Vulnerability. A Conceptual and Methodological Review. UNU-EHS SOURCE 4/2006, Bonn. 
Voigt-Graf, Carmen (2008): Transnationalism and the Ino-Fijian Diaspora: The Relationship of Indo-Fijians to India and its People. In: Journal of Intercultural Studies 29, 1, S. 81-109.

Waddington, Mark \& Giles Mohan (2004): Failing Forward: Going beyond PRA and Imposed Forms of Participation. In: Hickey, Samuel \& Giles Mohan (2004) Participation: from tyranny to transformation? Exploring new approaches to participation in development, London \& New York, S. 219-230.

Walsh, Catherine (2010): Development as Buen Vivir: Institutional arrangements and (de)colonial entanglements. In: Development 53, 1, S. 15-21.

Watson, Greg (2005): Conflict over Fiji. In: Henderson, John \& Greg Watson (Hg.): Securing a peaceful Pacific, S. 359-364, Christchurch.

WBGU (2011): Welt im Wandel: Gesellschaftsvertrag für eine Große Transformation. Hauptgutachten des Wissenschaftlichen Beirats der Bundesregierung Globale Umweltveränderungen, Berlin.

Weber, Eberhard (2007): Gewalt und Staatsversagen in Fidschi. In: Zeitschrift für Wirt-schaftsgeographie 51, 3/4, S. 206-220.

Weber, Eberhard (2013): Of tsunamis and climate change - the need to resettle. In: Andrianos, Lucas A., Sneep, Jan-Willem \& Guillermo Kerber (Hg.): Sustainable Alternatives for Poverty Reduction and Ecological Justice Vol. 1, S. 175-187.

Weber, Max (1917): Rußlands Übergang zum Scheinkonstitutionalismus. In: Archiv für Sozialwissenschaft und Sozialpolitik, 23, 1. In: ders. (1988): Gesammelte Politische Schriften, Tübingen.

Weber, Max (1919): Politik als Beruf. In: ders. (2002): Schriften: 1894 - 1922, Stuttgart, S. 474-511.

Weir, Christiane (2015): The 2014 Fiji Elections and the Methodist Church. In: The Round Table: The Commonwealth Journal of International Affairs, 104, 2, S. 165 175.

Weltbank (1997): Social capital: The missing link? Monitoring environmental progress - expanding the measure of wealth. Indicators and Environmental Valuation Unit (The World Bank, Environment Department), Chapter 6, Washington DC.

Weltbank (2013): World Development Indicators (WDI). World Bank database 02 July 2013.

Werlen, Benno (20083): Sozialgeographie. Eine Einführung, Stuttgart.

Werlen, Benno (2014): Globalisierung. In: Lossau, Julia, Freytag, Tim \& Roland Lippuner (Hg.): Schlüsselbegriffe der Kultur- und Sozialgeographie, Stuttgart, S. 54-66.

Williams, Thomas (1858): Fiji and the Fijians. The Islands and their Inhabitants. London.

Willis, Katie (2005): Theories and Practices of Development, London. 
Wise, Margaret (2014) Who will be in Cabinet. In: Fiji Times Online, 23.09.2014. http:/ / www.fijitimes.com/story.aspx?id=281038, 24.09.2014.

Wisner, Ben, Blaikie, Piers, Cannon, Terry \& Ian Davies $\left(2003^{2}\right)$ : At Risk. Natural hazards, people's vulnerability and disasters, New York.

Wolf, Marcus \& Michael Waibel (2010): Fidschi-Inseln. In: Dittmann, Andreas, Gieler, Wolfgang \& Matthias Kowasch (Hg.): Die Außenpolitik der Staaten Ozeaniens: ein Handbuch. Von Australien bis Neuseeland, von Samoa bis Vanuatu, Paderborn, S.43-52.

Wolfsberger, Judith $\left(2010^{3}\right)$ : Frei geschrieben. Mut, Freiheit und Strategie für wissenschaftliche Abschlussarbeiten, Wien, Köln \& Weimar.

Zelinsky, Wilbur (1971): The Hypothesis of the Mobility Transition. In: Geographical Review 61, S. 219-249. 


\section{Anhang: Fragebogen}

\section{Questionnaire: Role of Social Relations in Sustainable Human Development}

\section{A - Living Conditions}

A1 Please give me some background information on yourself

\begin{tabular}{|c|c|c|}
\hline Male & Female & Gender? \\
\hline Yes & No & Head of household? \\
\hline & & Age? (in years) \\
\hline & & Name of mataqali? \\
\hline & & Religion / Confession? \\
\hline & & Highest level of education (indicate in case you're still schooling) \\
\hline
\end{tabular}

A2 How many months did you stay outside the village in 2011 ? (if less than one month, put zero: " 0 ")

A2.1 In case you stayed away for one month or more, where did you live?

\begin{tabular}{|l|l|}
\hline & 1. other parts of Lomaiviti \\
\hline & 2. Viti Levu \\
\hline 3. other parts of Fiji \\
\hline 4. outside Fiji \\
\hline
\end{tabular}

A2.2 What were the purposes of staying

\begin{tabular}{|l|l|}
\hline away? \\
\hline & 1. work \\
\hline & 2. education \\
\hline & 3. business \\
\hline & 4. other: \\
\hline & 5. other: \\
\hline
\end{tabular}

A3 How many people currently live in your household?

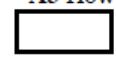

A3.1 Please split them into the following categories:

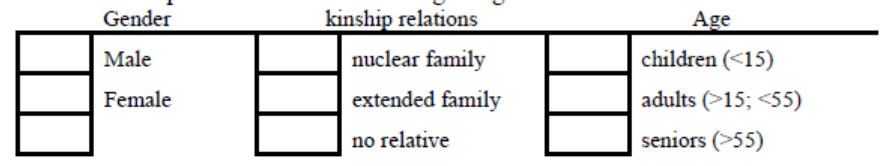

A4 Please rank the main sources of income for your household in 2011 (number 1 being most important)

\begin{tabular}{|l|l|}
\hline & income from wages \\
\hline & income from own farming and fishing activities \\
\hline & income from own non-agricultural activities (company) \\
\hline & income from own shop \\
\hline & money from family members \& friends living in your village \\
\hline & money from family members \& friends living outside your village \\
\hline & profits, dividends, interests on bank deposits, etc \\
\hline & Pensions \\
\hline & Rents \\
\hline & income from state assistance \\
\hline & other: \\
\hline
\end{tabular}




\section{B - Groups / Networks / Institutions / Associations}

B1 Please think of all the groups / networks / institutions / associations besides your family you belong to. Please tell me two important groups you're in to further discuss them with you:

I)

B1.1-I Please list some activities of the group and some benefits of it

\begin{tabular}{|l|l|}
\hline Activities & Benefits \\
\hline & \\
\hline & \\
\hline & \\
\hline & \\
\hline
\end{tabular}

B1.2-I How much time and money do you spend for the group?

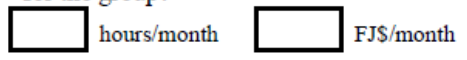

B.1.3-I Do you sometimes feel forced to spent time and/or money?

\begin{tabular}{|l|l|l|}
\hline & No & \\
\hline
\end{tabular}

B1.3-I How does one become a member?

\begin{tabular}{|l|l|}
\hline & 1. born into group \\
\hline & 2. ask to join group \\
\hline & 3. get invited \\
\hline & 4. prescribed/required to join \\
\hline & 5. other: \\
\hline
\end{tabular}

B1.4-I Does the group interact with other groups outside the village?

\begin{tabular}{|c|c|}
\hline & 1. no \\
\hline & 2. yes, occasionally \\
\hline & 3. yes, frequently \\
\hline
\end{tabular}

B1.5-I Has membership in the group changed during the last 10 years?

\begin{tabular}{|l|l|}
\hline & 1. declined \\
\hline & 2. remained the same \\
\hline & 3. increased \\
\hline
\end{tabular}

B1.6-I Who makes decisions?

\begin{tabular}{|l|l|}
\hline & 1. single leader \\
\hline & 2. small leading group \\
\hline & 3. every member \\
\hline & 4. outsider(s) \\
\hline & 5. other: \\
\hline & 6. combination of above \\
\hline
\end{tabular}

B1.7-I Do you think the way decisions are made is right?

\begin{tabular}{|l|l|}
\hline & 1. it's the right way \\
\hline & 2. it's a good way \\
\hline & 3. it needs to be improved \\
\hline & 4. it's a bad situation \\
\hline
\end{tabular}

II)

B1.1-II Please list some activities of the group and some benefits of it

\begin{tabular}{|l|l|}
\hline Activities & Benefits \\
\hline & \\
\hline & \\
\hline & \\
\hline & \\
\hline
\end{tabular}

B1.2-II How much time and money do you spend for the group?

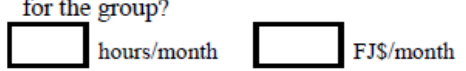

B.1.3-II Do you sometimes feel forced to spent time and/or money?

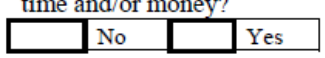

B1.3-II How does one become a member?

\begin{tabular}{|l|l|}
\hline & 1. born into group \\
\hline & 2. ask to join group \\
\hline & 3. get invited \\
\hline 4. prescribed/required to join \\
\hline 5. other:
\end{tabular}

B1.4-II Does the group interact with other groups outside the village?

\begin{tabular}{|l|l|}
\hline & 1. no \\
\hline & 2. yes, occasionally \\
\hline & 3. yes, frequently \\
\hline
\end{tabular}

B1.5-II Has membership in the group changed during the last 10 years?

\begin{tabular}{|l|l|}
\hline & 1. declined \\
\hline & 2. remained the same \\
\hline & 3. increased \\
\hline
\end{tabular}

B1.6-II Who makes decisions?

\begin{tabular}{|l|l|}
\hline & 1. single leader \\
\hline & 2. small leading group \\
\hline 3. every member \\
\hline 4. outsider(s) \\
\hline 5. other: \\
\hline 6. combination of above \\
\hline
\end{tabular}

B1.7-II Do you think the way decisions are made is right?

\begin{tabular}{|l|l|}
\hline & 1. it's the right way \\
\hline & 2. it's a good way \\
\hline & 3. it needs to be improved \\
\hline & 4. it's a bad situation \\
\hline
\end{tabular}


B2 Are there groups existing you want to join but you're not allowed to?



B2.1 If yes, please specify the group and give reasons why you're not allowed to:

\begin{tabular}{|l|l|}
\hline Group & Reason \\
\hline & \\
\hline & \\
\hline & \\
\hline
\end{tabular}

\section{C - Trust, Solidarity \& Cooperation}

\begin{tabular}{|c|c|c|c|c|}
\hline $\begin{array}{l}\text { Cl To what extent do you trust the people in the following } \\
\text { categories? }\end{array}$ & 承焉 & 胥 & 总 & 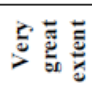 \\
\hline \multicolumn{5}{|l|}{ family members } \\
\hline \multicolumn{5}{|l|}{ village community members (without family) } \\
\hline \multicolumn{5}{|l|}{ village leader } \\
\hline \multicolumn{5}{|l|}{ Police } \\
\hline \multicolumn{5}{|l|}{ Judges } \\
\hline \multicolumn{5}{|l|}{ local government officials } \\
\hline \multicolumn{5}{|l|}{ National government officials } \\
\hline \multicolumn{5}{|l|}{ members of non-governmental organisations } \\
\hline Strangers & & & & \\
\hline
\end{tabular}

C2 How well do people in your community help each other out these days?

\begin{tabular}{|l|l|}
\hline & 1. always helping \\
\hline & 2. helping most of the time \\
\hline & 3. helping sometimes \\
\hline & 4. rarely helping \\
\hline
\end{tabular}

C3 Do you think that over the past 10 years the solidarity in your community has gotten better, worse, or stayed the same?

\begin{tabular}{|l|l|}
\hline & 1. better \\
\hline & 2. stayed the same \\
\hline & 3. worse \\
\hline
\end{tabular}

C4 What proportion of people in this village contributes time or money toward common development goals (e.g. like solving a water supply problem)?

\begin{tabular}{|l|l|}
\hline & 1. everyone \\
\hline & 2. more than half \\
\hline & 3. about half \\
\hline & 4. less than half \\
\hline & 5. no one \\
\hline
\end{tabular}

C4.1 If not everyone, is it possible to identify certain groups within the community who are excluded from contributions?

C5 In case of a serious emergency that you can hardly handle within the community (e.g. like cyclone Tomas in March 2010), in your opinion, form whom does your village community get most support? Please rank the most important.

\begin{tabular}{|l|l|l|l|}
\hline & neighbouring villages & & Government \\
\hline & friends \& relatives further away & & non-governmental humanitarian organisations \\
\hline & Church & & other (specify): \\
\hline
\end{tabular}


C6 In general, how safe from crimes or violence do you feel when being home alone?

\begin{tabular}{|l|l|}
\hline & 1. very safe \\
\hline & 2. safe \\
\hline & 3. moderately safe \\
\hline & 4. moderately unsafe \\
\hline & 5. unsafe \\
\hline & 6. very unsafe \\
\hline
\end{tabular}

C7 In the last 12 month, have you or anyone of your household been the victim of a crime or violence?

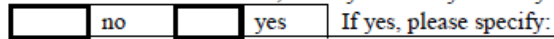

C8 Within the last 10 years, do you think the level of violence in your community changed?

\begin{tabular}{|l|l|}
\hline & 1. better (level of violence decreased) \\
\hline & 2. stayed the same \\
\hline & 3. worse (level of violence increased) \\
\hline
\end{tabular}

\section{D - Political participation}

D1 How much impact do you think you have in decisions that affect your life?

\begin{tabular}{|l|l|}
\hline & 1. huge impact \\
\hline & 2. small impact \\
\hline & 3. no impact \\
\hline
\end{tabular}

D1.1 Do you feel satisfied with this situation?

\begin{tabular}{|l|l|}
\hline & 1. yes, to a great extent \\
\hline 2. only to a small extend \\
\hline 3. no, it's a bad situation \\
\hline
\end{tabular}

D2 Did you vote in the last national election?

\begin{tabular}{|l|l|}
\hline & no \\
\hline
\end{tabular}

D3 Does your village ask government officials for something benefitting the community?

\begin{tabular}{|l|l|}
\hline & 1. no \\
\hline & 2. yes, occasionally \\
\hline & 3. yes, frequently \\
\hline
\end{tabular}

D 3.1 If yes, are any of these petitions successful?

\begin{tabular}{|l|l|}
\hline & 1. almost always \\
\hline & 2. often \\
\hline & 3. seldom \\
\hline & 4. never \\
\hline
\end{tabular}

\section{E - Communication \& Information}

E1 How many times in a month do you or anyone in your household read a newspaper?

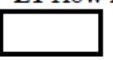

E2 How often do you listen to the radio?

\begin{tabular}{|l|l|}
\hline & 1. everyday \\
\hline & 2. a few days a week \\
\hline & 3. once a week \\
\hline & 4. less than once a week \\
\hline
\end{tabular}

E3 How many times in a month do you make or receive a phone call? 
Bisher erschienene Bände der Reihe Publications of the book series

FINK, M. (2016): Soziale Sicherung im Spannungsfeld gesellschaftlicher Transformation. Eine partizipative Studie in Küstendörfern der Fidschi-Inseln. In: Schriftenreihe PAZIFIK FORUM der Arbeitsgemeinschaft für Pazifische Studien e.V. (Hrsg.: Dittrich, C., Faust, H. \& M. Waibel); Band 17, Universitätsverlag Göttingen 2016. Göttingen, 254 S.

WAIBEL, M. (ED.) (2016): Đà Nẵng: COASTAL City. Sach anh / Photo Book. 1st edition. Book series PAZIFIK FORUM of the Association of Pacific Studies; Volume 16. Fine Art Publishing House, Hanoi / Vietnam, $302 \mathrm{p}$.

WAIBEL, M. \& H. HILBERT (EDS.) (2015): TP. Ho Chi Minh: MEGA City. Sach anh / Photo Book. 2nd updated edition. Book series PAZIFIK FORUM of the Association of Pacific Studies; Volume 15. Fine Art Publishing House, Hanoi / Vietnam, 208 p.

WAIBEL, M. (HRSG.) (2013): Ho Chi Minh MEGA City. Schriftenreihe PAZIFIK FORUM der Arbeitsgemeinschaft für Pazifische Studien e.V.; Band 14, Regiospectra Verlag, Berlin 2013, 274 S.

SCHNEIDER, H., JORDAN, R. \& M. WAIBEL (HRSG.) (2012): Umweltkonflikte in Südostasien. Schriftenreihe PAZIFIK FORUM der Arbeitsgemeinschaft für Pazifische Studien e.V.; Band 13, Horlemann Verlag, Berlin 2012, 240 S.

WEBER, R. (2006): Kulturlandschaftswandel in Zentralsulawesi - Historisch-geographische Analyse einer indonesischen Bergregenwaldregion. In: Schriftenreihe PAZIFIK FORUM der Arbeitsgemeinschaft für Pazifische Studien e.V. (Hrsg.: Faust, H., Kreisel, W. \& M. Waibel); Band 12, Universitätsverlag Göttingen 2006. Göttingen, $238 \mathrm{~S}$.

WAIBEL, M., JORDAN, R. \& H. SCHNEIDER (HRSG.) (2006): Krisenregion Südostasien - Alte Konflikte und neue Kriege. Schriftenreihe PAZIFIK FORUM der Arbeitsgemeinschaft für Pazifische Studien e.V.; Band 11, Horlemannverlag, Bad Honnef 2006. 172 S.

WAIBEL, M. \& W. KREISEL (EDS.) (2005): The Pacific Challenge - Development Trends in the 21st century. Schriftenreihe PAZIFIK FORUM der Arbeitsgemeinschaft für Pazifische Studien e.V.; Band 10, Universitätsverlag Göttingen, Göttingen 2005. 176 S.

WAIBEL, M., THIMM, T. \& W. KREISEL (HRSG.) (2005): Fragile Inselwelten - Tourismus, Umwelt und indigene Kulturen. Schriftenreihe PAZIFIK FORUM der Arbeitsgemeinschaft für Pazifische Studien e.V.; Band 9, Horlemannverlag, Bad Honnef 2005. 256 S.

KREISEL, W., MARSDEN, P.H.\& M. WAIBEL (HRSG.) (2003): Wandel, Werte und Wirtschaft im pazifischen Raum. Schriftenreihe PAZIFIK FORUM der Arbeitsgemeinschaft für Pazifische Studien e.V.; Band 8, Duehrkohp \& Radicke, Göttingen 2003. 232 S.

THIMM, T. (2002): Kulturwandel und Tourismus in Französisch-Polynesien. In: Schriftenreihe PAZIFIK FORUM der Arbeitsgemeinschaft für Pazifische Studien e.V. (Hrsg.: Kreisel, W. \& M. Waibel); Band 7, Duehrkohp \& Radicke, Göttingen 2002. 230 S.

FAUST, H. (1996): Verstädterung in Fiji - Besonderheiten in den Stadt-Land-Beziehungen eines insularen pazifischen Entwicklungslandes. Schriftenreihe PAZIFIK FORUM der Arbeitsgemeinschaft Pazifische Studien Aachen e.V.; Band 6, Aachen 1996. 226 S.

KREISEL, W. \& P.H. MARSDEN (HRSG.): "First Peoples, First Voices": Indigene Völker zwischen Fremdbestimmung und Selbstbehauptung. Schriftenreihe PAZIFIK FORUM der Arbeitsgemeinschaft für Pazifische Studien Aachen e. V.; Band 5, Aachen 1995. 236 S.

KREISEL, W., VOSSEN, J. \& F. DICKMANN (HRSG.): Wirtschaft und Umwelt im asiatisch-pazifischen Raum. Schriftenreihe PAZIFIK FORUM der Arbeitsgemeinschaft für Pazifische Studien Aachen e. V.; Band 4, Aachen 1995. 204 S.

KREISEL, W., MARSDEN P.H., DAVIS, G. V. \& J. JANSEN (HRSG.): Neuseeland im Pazifischen Raum. Schriftenreihe PAZIFIK FORUM der Arbeitsgemeinschaft für Pazifische Studien Aachen e. V.; Band 3, Aachen 1992.

KREISEL, W., VOSSEN, J. \& F. DICKMANN (HRSG.): Der Tourismus in der pazifischen Inselwelt. Schriftenreihe PAZIFIK FORUM der Arbeitsgemeinschaft für Pazifische Studien Aachen e. V.; Band 2, Aachen 1991.

KREISEL W., VOSSEN, J. \& F. DICKMANN (HRSG.): Entwicklungstendenzen und Entwicklungsstrategien im pazifischen Inselraum. Schriftenreihe PAZIFIK FORUM der Arbeitsgemeinschaft für Pazifische Studien Aachen e. V.; Band 1, Aachen 1990. 

Issue \#45 | January / February 2016 | www.pacific-geographies.org | ISSN 2196-1468 | 6 6,00

\section{Pacific Geographies}

Research | Notes | Current Issues from the Asia-Pacific Region

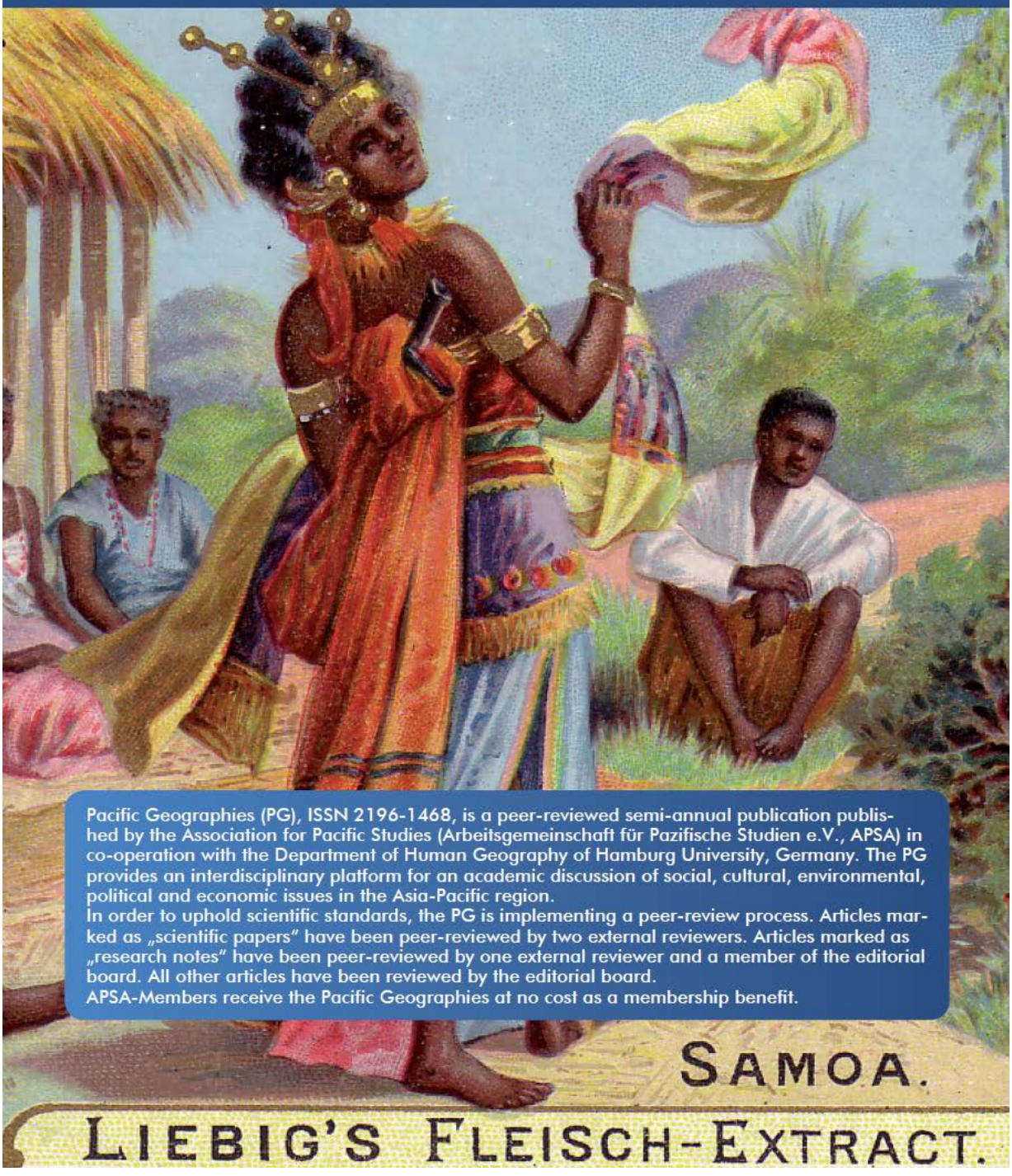


Pazifikstaaten wie die Fidschi-Inseln sind wie kaum eine andere Region der Welt gekennzeichnet von gesellschaftlichen Veränderungen und dem globalen Umweltwandel. Auf Basis eines partizipativen Forschungsdesigns, durchgeführt in ausgewählten Küstendörfern, untersucht dieser Band die Auswirkungen der Globalisierung und des Klimawandels auf die Lebensqualität ihrer Bewohner. Dabei wird aufgezeigt, dass die Dorfbewohner keine passiven Opfer externer Prozesse sind, sondern aktiv ihr Leben gestalten. Mittels neo-traditioneller Bewegungen gelingt es den Bewohnern weitestgehend, ein festes Wertefundament als Grundlage ihrer kulturellen Identität zu bewahren. Eine intakte Gemeinschaft steht dabei im Zentrum spiritueller, ökonomischer und sozialer Werte.

Im Zuge der gesellschaftlichen Transformation agieren die Gemeinschaften nicht mehr ortsgebunden, sondern bilden mithilfe moderner Transport- und Kommunikationstechnologien globalisierte Netzwerke - ohne dabei die Bezüge zu ihrer kulturellen Herkunft zu verlieren. Auswärts lebende Familienangehörige tragen so Wissen und materiellen Wohlstand in die Dorfgemeinschaften und erweitern die Handlungspotentiale und Lebensstandards der Küstendorfbewohner. Im Zuge der Wissensvermittlung erfolgt jedoch insbesondere die Darstellung des Klimawandels und seiner Gefahren verkürzt und nicht auf lokale Werte zurechtgeschnitten, sodass es auch zu Wahrnehmungsverzerrungen und Fehlanpassungen kommt. Im Kontext von Entwicklungszusammenarbeit rät diese Untersuchung daher zu kultureller Sensibilität und einer kritischen Reflektion ethnozentrischer Weltanschauungen, um die Lebensqualität in fidschianischen Dorfgemeinschaften nicht zu gefährden.

In diesem Band werden ethnographische Ideale für eine kultur- und sozialgeographische Forschung im globalen Süden aufbereitet. Konzeptionell ist die Untersuchung in die kritische geographische Verwundbarkeits- und Transformationsforschung eingerahmt. In einem kulturräumlichen Überblick wird zunächst aus historisch-politischer Perspektive die Bedeutung postkolonialer Belastungen auf gegenwärtige Transformationsprozesse aufgezeigt. Das Herzstück bildet anschließend die ausführliche Analyse gesellschaftlicher Transformationsprozesse auf die Lebensqualität in drei Küstendörfern der FidschiInseln.

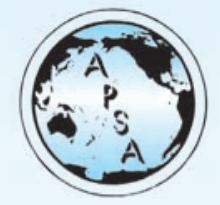

